University of Tennessee Health Science Center UTHSC Digital Commons

\title{
Multipronged Approach to Study Glaucoma-Associated Phenotypes
}

Sumana Rameshbabu Chintalapudi

University of Tennessee Health Science Center

Follow this and additional works at: https://dc.uthsc.edu/dissertations

Part of the Neurosciences Commons

\section{Recommended Citation}

Chintalapudi, Sumana Rameshbabu (http://orcid.org/0000-0003-1079-0950), "Multipronged Approach to Study Glaucoma-Associated Phenotypes" (2016). Theses and Dissertations (ETD). Paper 404. http://dx.doi.org/10.21007/etd.cghs.2016.0410. 


\title{
Multipronged Approach to Study Glaucoma-Associated Phenotypes
}

\begin{abstract}
Glaucoma refers to a group of conditions characterized by death of RGCs, increase in intraocular pressure is usually a precursor to glaucoma and irreversible optic neuropathy leads to visual impairment and blindness. Recent advances have seen a surge of new ideas and technologies to aid in the early detection, efficacious treatments and neuroprotection. Despite advances we face several challenges in understanding the pathophysiology of glaucoma. One of the many challenges scientists and ophthalmologists are facing is, to better understand IOP, its role in glaucomatous damage and design safer, more predictable IOP-lowering therapies. Another challenge is to find a practical method or develop tools to understand the molecular pathways to study RGC function and health to develop novel therapies for vision loss. This work leads to

three complementary insights on how to address these challenges. First, we used a novel systems genetics approach to identify and validate genetic modifiers of IOP using the enlarged BXD family of strains in combination with human GWAS glaucoma cohorts. This will pave the way for improved drug development tailored to individual genotypes for POAG. Second, we have combined systems genetics, bidirectional studies using multiple species, meta-analyses, immunohistochemistry, FACS sorting and gene knockdown studies to identify and validate the identity of a genetic modulator of Sncg, a gene that has been previously implicated in RGC death in glaucoma. Outcomes of the investigation may provide clues to understanding the molecular mechanisms that account for the degenerative changes in RGCs in glaucoma. Third, we optimized a feasible, reproducible, standardized flow cytometry-based protocol for the isolation and enrichment of homogeneous RGC. This will allow for future careful assessment of important cell specific pathways in RGC to provide mechanistic insights into the declining of visual acuity in aged populations and those suffering from retinal neurodegenerative diseases. Taken together these studies offer a concise outlook on use of integrated systems science, molecular and imaging technologies as a needs-led innovation in ophthalmology and visual health.
\end{abstract}

\section{Document Type}

Dissertation

Degree Name

Doctor of Philosophy (PhD)

\section{Program}

Integrated Program in Biomedical Sciences

\section{Research Advisor}

Monica M. Jablonski, Ph.D.

\section{Keywords}

Flow cytometry, Glaucoma, Intraocular pressure, Retina, Retinal ganglion cells, Systems genetics

\section{Subject Categories}

Medical Sciences | Medicine and Health Sciences | Neurosciences

\section{Comments}

One year embargo expires August 2017. 


\title{
Multipronged Approach to Study Glaucoma-Associated Phenotypes
}

\author{
A Dissertation \\ Presented for \\ The Graduate Studies Council \\ The University of Tennessee \\ Health Science Center
}

In Partial Fulfillment

Of the Requirements for the Degree

Doctor of Philosophy

From The University of Tennessee

By

Sumana Rameshbabu Chintalapudi

August 2016 
Chapter 3 (C) 2016 by John Wiley \& Sons.

Chapter 4 (C) 2016 by 2007-2016 Frontiers Media, SA.

All other material (C) 2016 by Sumana Rameshbabu Chintalapudi.

All rights reserved. 


\section{DEDICATION}

To my parents,

Ramesh Babu Chintalapudi

Usha Rani Chithirala

And

My better half,

Purav Trivedi 


\section{ACKNOWLEDGEMENTS}

First of all, I wish to thank the Almighty Lord for helping me start, pursue and successfully complete my $\mathrm{PhD}$. Working as $\mathrm{PhD}$ student in UTHSC was a magnificent as well as challenging experience for me. This dissertation was the product of a large measure of serendipity, fortuitous encounters with people who have changed the course of my academic career. It would not have been possible without the help of so many people in so many ways. Here is a tribute to all those people.

I want to thank my supervisor Prof. Dr. Monica M. Jablonski for giving me this wonderful opportunity to pursue $\mathrm{PhD}$ and introducing me to the world of glaucoma research. I will cherish the time I have spent as your mentee for the rest of my life. The academic and research freedom you provided me is unparalleled, and the intellectually stimulating discussions regarding work and research, and conversations about all things non-academic have benefitted me immensely. Above all and the most needed, she provided me unflinching encouragement and support in various ways. Dr. Vanessa Morales-Tirado has been supportive throughout the doctoral program, her cheerful enthusiasm and ever-friendly nature was a constant inspiration, she has provided tremendous help in the study design and analytical methodology in my research studies. Dr. Robert Williams has always asked thought-provoking questions and provided insightful feedback that has helped me in improving my research studies. Dr. Dianna Johnson has been kind to meet me several times on short notice and provided great feedback and inputs regarding research and life in academics. Dr. Edward Chaum has provided great feedback and inputs for my research studies during the vision research seminars and committee meetings. Dr. Rajender Raghow has been very supportive and provided valuable guidance to improve my research studies.

My wonderful family stood by me and encouraged me in each and every endeavor that I have embarked upon. With enduring patience, they stood by me during the ups and downs of my life. I can barely find words to express all the wisdom, love and support given to me by my beloved parents, Dr. Ramesh Babu Chintalapudi and Mrs. Usha Rani Chithirala for their unconditional love, fidelity, endurance and encouragement. I am deeply indebted to my best friend and husband, Purav Trivedi, for giving me untiring support during my years of Doctoral study. I am wordless in expressing my sincere gratefulness for his constant encouragement and love. He has helped me to see and practice life altogether in a different perspective, to stay focused and to go after my dreams. I thank my brother Anil Kumar Chintalapudi and his wife Leena Kora for their unconditional love and support at all times in life, and especially during my doctoral education. I would like to thank my wonderful in-laws, Mr. Tuhinanshu Trivedi and Mrs. Meha Trivedi, for their love and support, and blessings during this critical phase in my life. I thank my sister-in-law, Pankti Trivedi, for her support and affection.

Many friends and colleagues from both India and US have helped me stay sane throughout my $\mathrm{PhD}$. Their support and care helped me overcome setbacks and stay focused on my graduate study. I greatly value their friendship and I deeply appreciate 
their belief in me. I want to thank current and past members of Jablonski, XiangDi Wang, Doaa Maria, Mohamed Mostafa Ibrahim, Samy Maria, Yunfeng Shi, Mallika Palamoor, Liyuan Li, Huiling Li, Hong Lu, Xiaofei Wang. Also to members of Morales lab, Bradley Gao, Levon Djenderedjian and Zachary Goldsmith.

My tribute....to a number of animals who have paid a price with their lives and suffering in the name of human protection. I pay my tribute to their sacrifice and pray that it is not in vain.

In all, the journey to my Ph.D. has been a long and challenging road. And I am thrilled it is done. But most of all, I am excited for what the future holds.... 


\begin{abstract}
Glaucoma refers to a group of conditions characterized by death of RGCs, increase in intraocular pressure is usually a precursor to glaucoma and irreversible optic neuropathy leads to visual impairment and blindness. Recent advances have seen a surge of new ideas and technologies to aid in the early detection, efficacious treatments and neuroprotection. Despite advances we face several challenges in understanding the pathophysiology of glaucoma. One of the many challenges scientists and ophthalmologists are facing is, to better understand IOP, its role in glaucomatous damage and design safer, more predictable IOP-lowering therapies. Another challenge is to find a practical method or develop tools to understand the molecular pathways to study RGC function and health to develop novel therapies for vision loss. This work leads to three complementary insights on how to address these challenges. First, we used a novel systems genetics approach to identify and validate genetic modifiers of IOP using the enlarged BXD family of strains in combination with human GWAS glaucoma cohorts. This will pave the way for improved drug development tailored to individual genotypes for POAG. Second, we have combined systems genetics, bidirectional studies using multiple species, meta-analyses, immunohistochemistry, FACS sorting and gene knockdown studies to identify and validate the identity of a genetic modulator of Sncg, a gene that has been previously implicated in RGC death in glaucoma. Outcomes of the investigation may provide clues to understanding the molecular mechanisms that account for the degenerative changes in RGCs in glaucoma. Third, we optimized a feasible, reproducible, standardized flow cytometry-based protocol for the isolation and enrichment of homogeneous RGC. This will allow for future careful assessment of important cell specific pathways in RGC to provide mechanistic insights into the declining of visual acuity in aged populations and those suffering from retinal neurodegenerative diseases. Taken together these studies offer a concise outlook on use of integrated systems science, molecular and imaging technologies as a needs-led innovation in ophthalmology and visual health.
\end{abstract}




\section{TABLE OF CONTENTS}

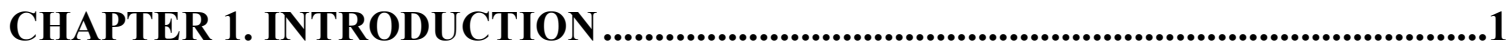

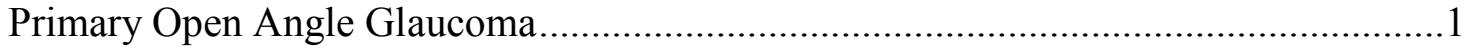

IOP as a major risk factor .......................................................................... 1

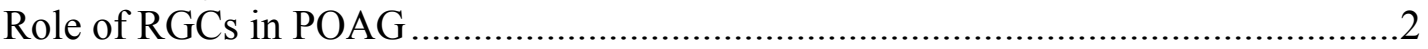

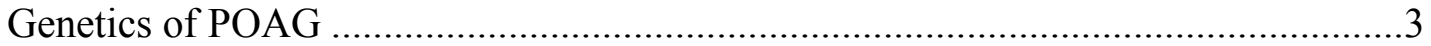

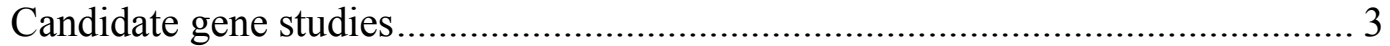

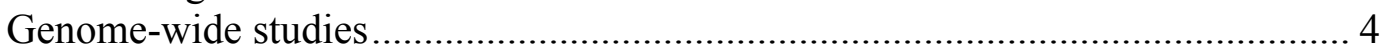

Systems Genetics Approach to Study Glaucoma .......................................................

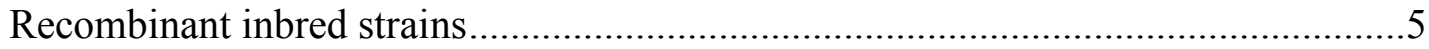

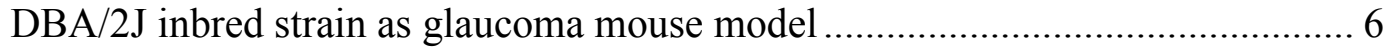

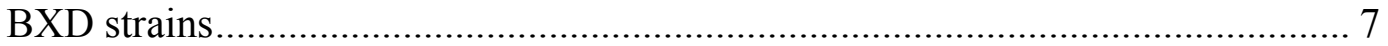

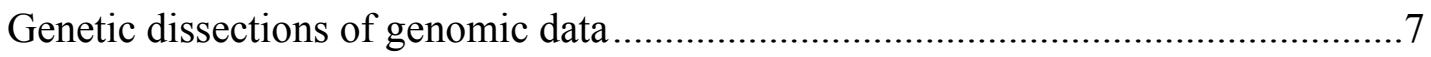

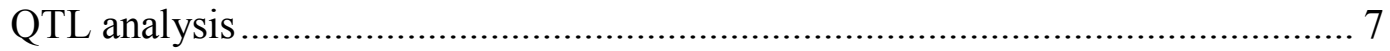

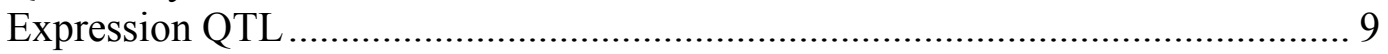

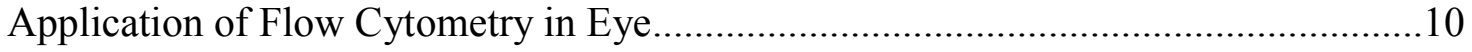

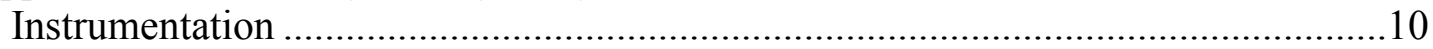

Flow cytometry application in ocular diseases and retinal cell biology ...................11

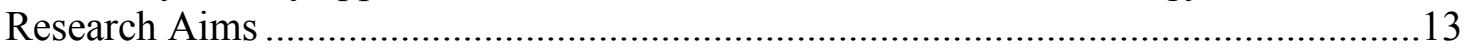

Aim 1: To identify the candidate gene that modulates IOP in mouse and test translational validity of the mouse candidates using the cohorts of human

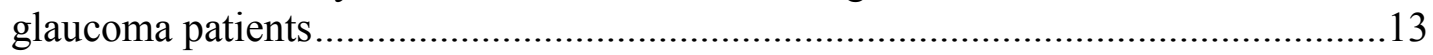

Aim 2: To identify and validate regulator of Sncg in primary mouse RGCs.............13

Aim 3: To isolate and validate primary murine RGCs from healthy and recombinant inbred mouse model of elevated IOP

\section{CHAPTER 2. SYSTEMS GENETICS APPROACH TOWARDS} UNCOVERING THE GENE MODULATORS OF IOP ............................................14

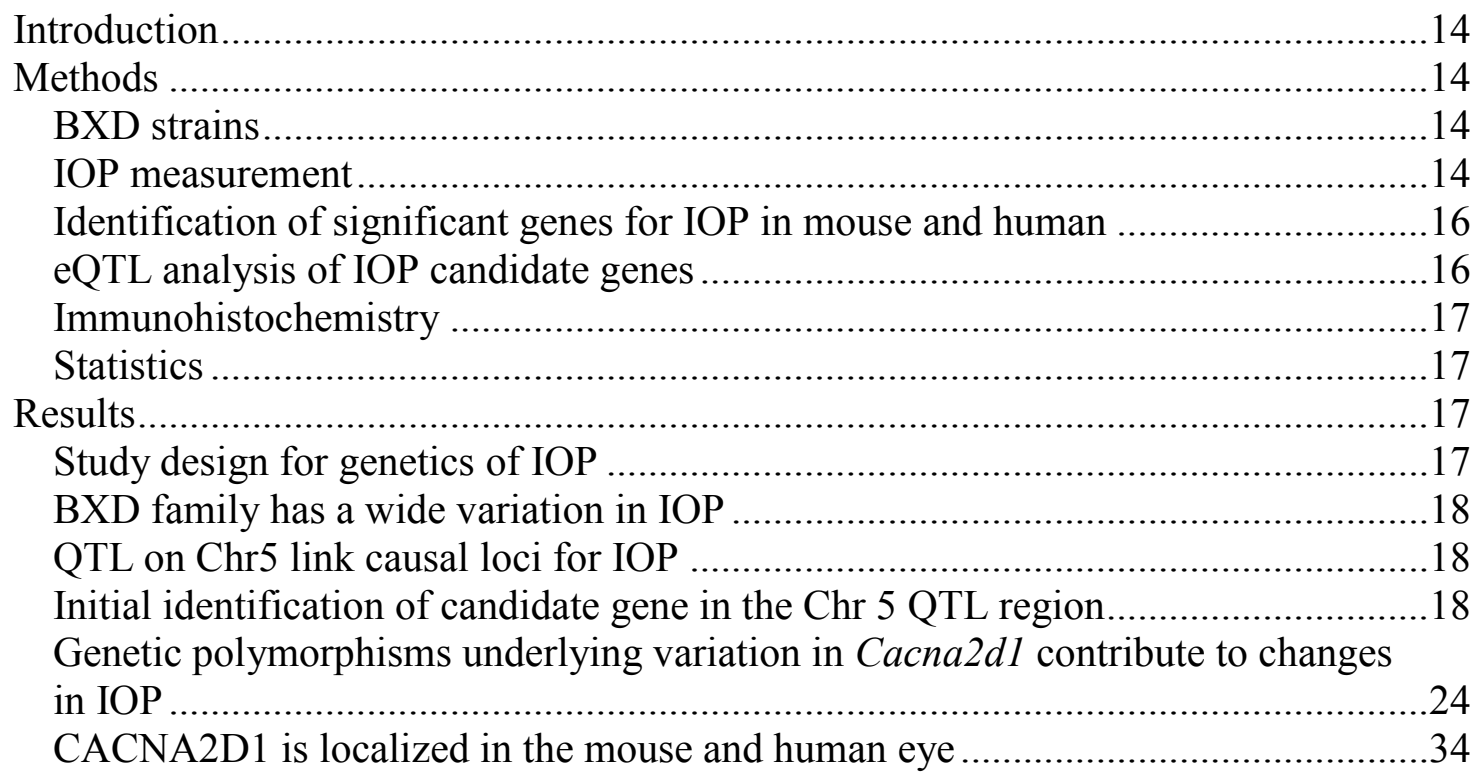


Discussion

\section{CHAPTER 3. MULTIPRONGED APPROACH TO IDENTIFY AND VALIDATE A NOVEL UPSTREAM REGULATOR OF SNCG IN MOUSE

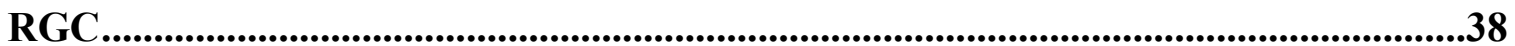

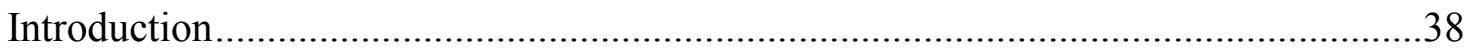

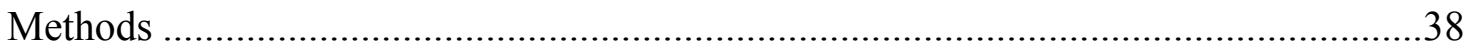

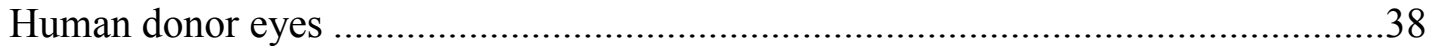

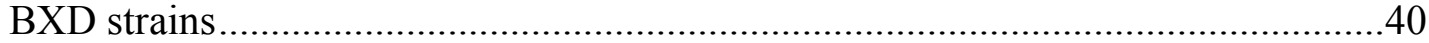

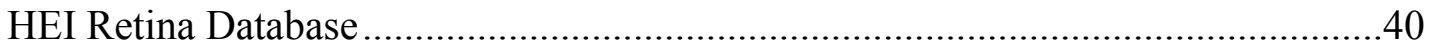

eQTL mapping and heritability calculation ..................................................... 41

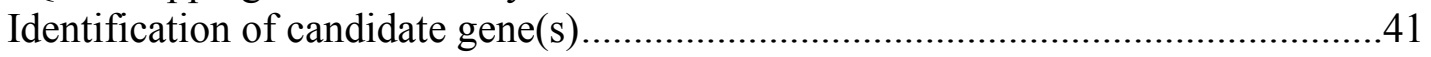

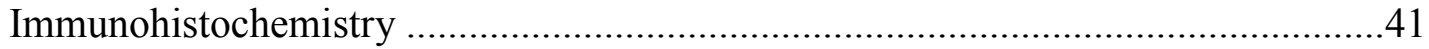

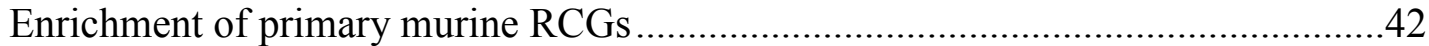

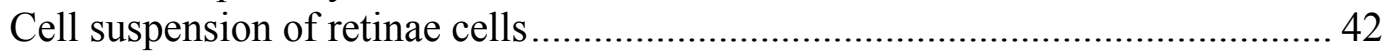

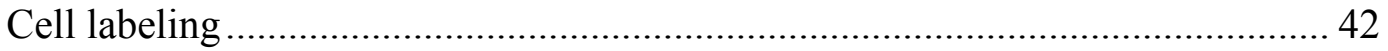

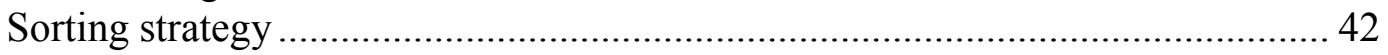

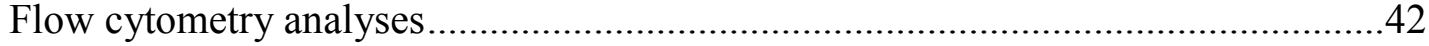

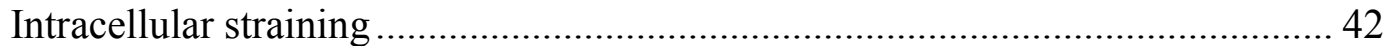

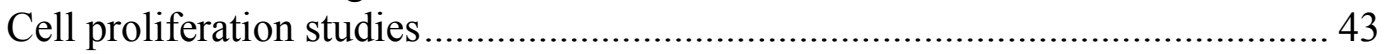

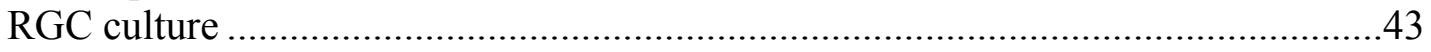

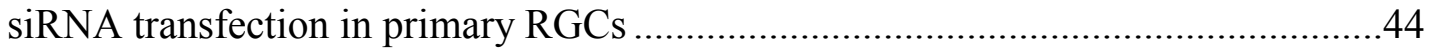

RNA isolation, cDNA synthesis and quantitative polymerase chain reaction...........44

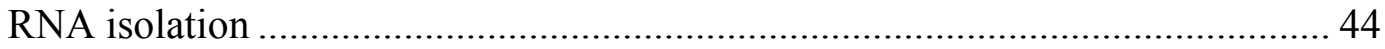

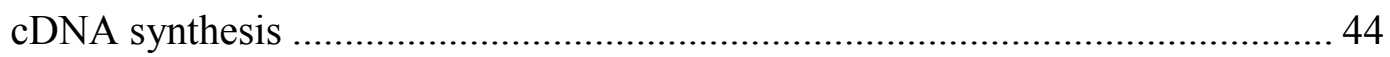

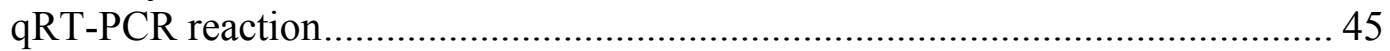

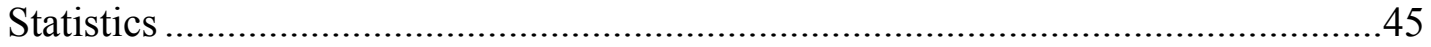

Correlation comparison and gene ontology tree machine construction ...................45

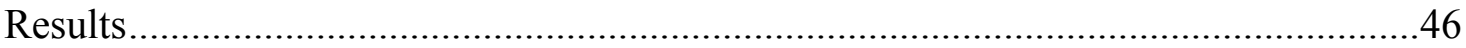

Identification of $P f d n 2$ as a candidate gene modulating $S n c g$ expression in

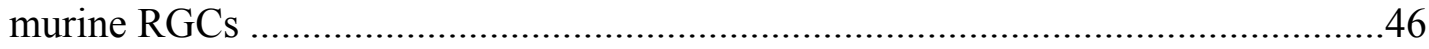

Subcellular localization of SNCG and PFDN2 demonstrate cytosolic colocalization in mouse and human RGCs ...........................................................52

Quantitative and qualitative analyses of SNCG and PFDN2 in isolated RGCs ........56

siRNA mediated knockdown of $P f d n 2$ significantly reduced the expression

of Sncg in enriched RGCs.........................................................................6

Network enrichment highlights the involvement of mitochondrial function............60

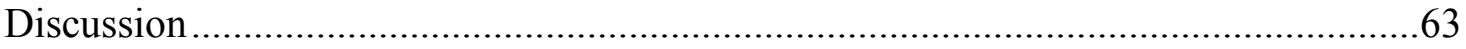

\section{CHAPTER 4. ISOLATION AND VALIDATION OF PRIMARY MURINE} RGCS FROM HEALTHY AND DAMAGED RETINAE ......................................69

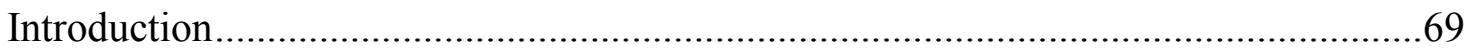

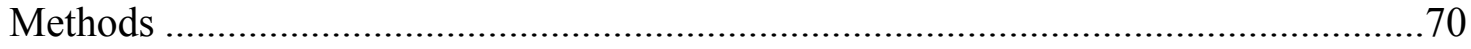

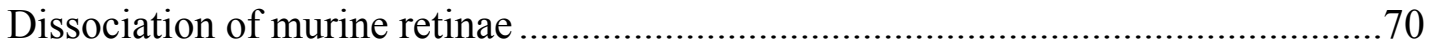

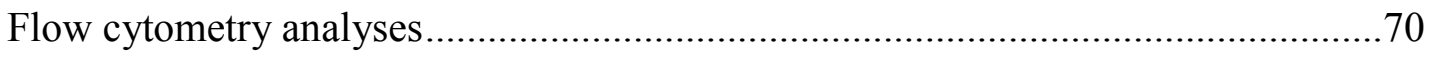

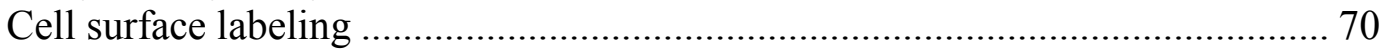




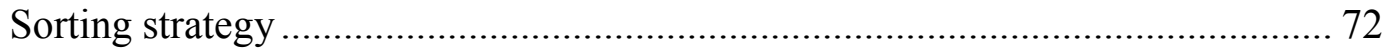

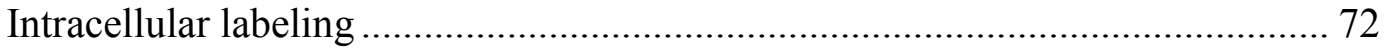

Flow cytometry and confocal microscopy dual analysis .................................... 72

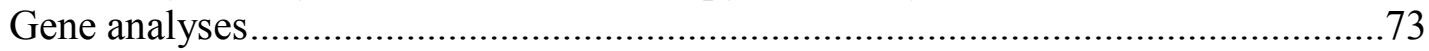

RNA isolation and cDNA synthesis and pre-amplification of cDNA template ... 73

qPCR analysis amplification efficiency test ......................................................... 73

qPCR reaction .......................................................................................... 73

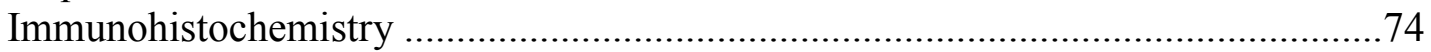

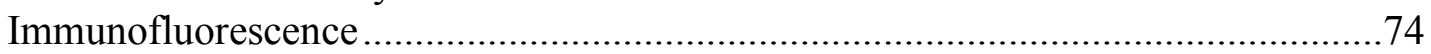

Optic nerve processing, imaging and counting .....................................................75

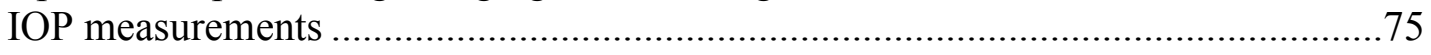

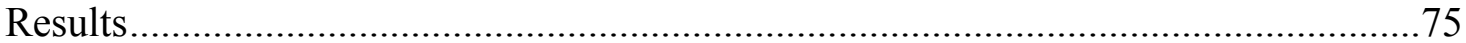

The Thy $1^{+} \mathrm{CD} 48^{\text {neg }}$ surface phenotype is not sufficient to identify murine RGCs ....75

Confirmation of additional surface markers to be used as negative selectors and

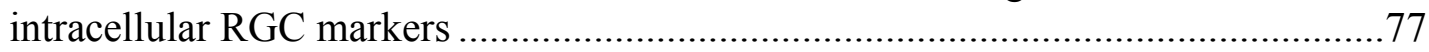

Live Thy $1.2^{+} \mathrm{CD} 48^{\text {neg }} \mathrm{CD} 15^{\text {neg }} \mathrm{CD} 57^{\text {neg }} \mathrm{RGC}$ express all signature intracellular

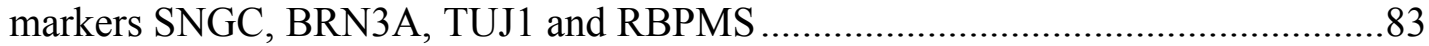

Validation of the enriched RGC population by qPCR analyses .................................83

Highly enriched RGCs can be isolated from a mouse model with elevated IOP and optic nerve damage......................................................................................99

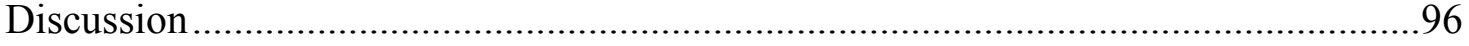

CHAPTER 5. DISCUSSION AND CONCLUSION......................................................99

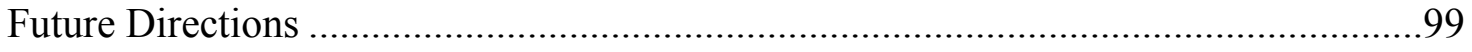

Aim1: To identify the candidate gene that modulates IOP in mouse and test translational validity of the mouse candidates using the cohorts of human glaucoma patients .99 Aim 2: To identify and validate regulator of Sncg in primary mouse RGCs...........101 Aim 3: To isolate and validate primary murine RGCs from healthy and recombinant inbred mouse model of elevated IOP

Glaucomics-Future of Glaucoma Research

LIST OF REFERENCES ................................................................................................107

APPENDIX A. SUPPLEMENTAL DATA FOR CHAPTER 2....................................123

APPENDIX B. SUPPLEMENTAL DATA FOR CHAPTER 3 ...................................127

APPENDIX C. SUPPLEMENTAL DATA FOR CHAPTER 4..................................135

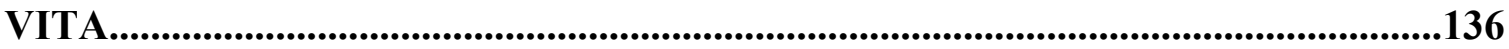




\section{LIST OF TABLES}

Table 2-1. Summary of candidate gene Cacna2d1 in mouse .....................................33

Table 2-2. Summary of candidate gene Cacna2d1 in humans....................................33

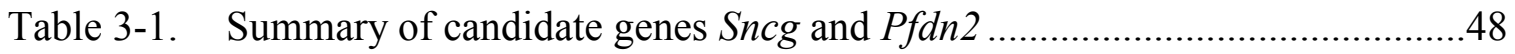

Table 4-1. Genes used in gene expression analyses as part of the validation of our

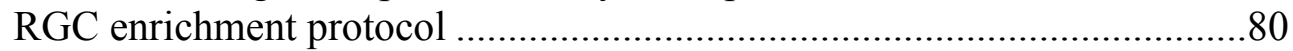

Table A-1. IOP values of BXD strains at 9.1-13 months age ..................................123

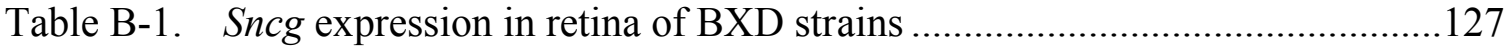

Table C-1. List of primers used for gene expression analysis as a component of the validation of RGC enrichment protocol ...................................................135 


\section{LIST OF FIGURES}

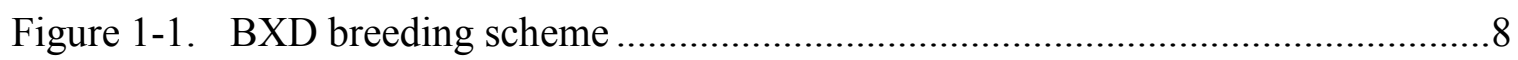

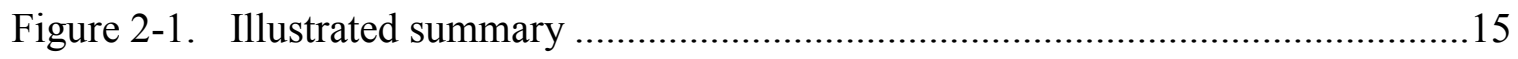

Figure 2-2. IOP levels across BXD strains and parental strains ......................................19

Figure 2-3. Tyrp1 and Gpnmb genotypes do not influence IOP ....................................20

Figure 2-4. Correlation of IOP for females and males at 9.1-13 months .......................21

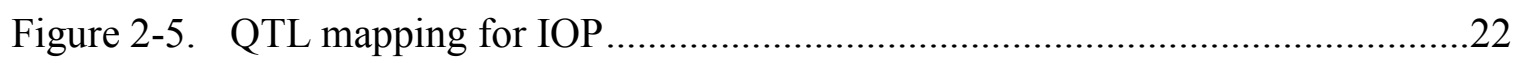

Figure 2-6. QTL mapping for Tyrp1 and Gpnmb in BXD strains eyes..........................23

Figure 2-7. The screen shot depicts the Pearson correlation analysis using the tools available on GeneNetwork ........................................................................25

Figure 2-8. The screen shot depicts the partial correlation analysis using the tools

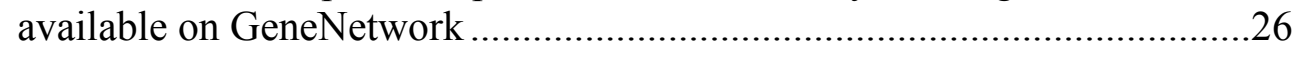

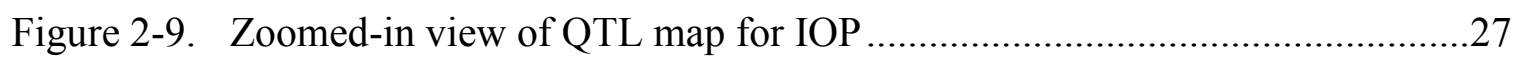

Figure 2-10. Subunit structure of L type calcium channel with description. …................28

Figure 2-11. mRNA rxpression levels of Cacna2dl across BXD strains and parental

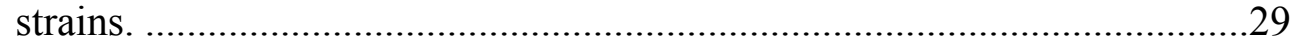

Figure 2-12. A significant cis-eQTL for Cacna2d1 is present on chromosome 5 at 14$19 \mathrm{Mb}$.

Figure 2-13. Cacna2dl expression in whole eye of BXD strains attributable to the genotype

Figure 2-14. UCSC Genome Browser illustration of the gene structure and location of SNPs associated with Cacna2dl gene

Figure 2-15. Cellular localization of CACNA2D1 in C57BL/6J mouse eye

Figure 2-16. Cellular localization of CACNA2D1 in a human donor eye with no diagnosis of ocular diseases

Figure 3-1. Illustrated summary

Figure 3-2. mRNA expression levels of Sncg across BXD strains and parental strains 


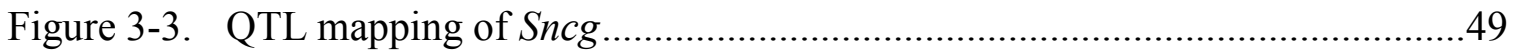

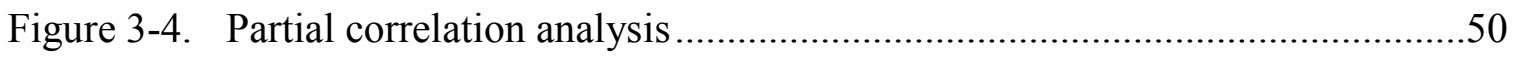

Figure 3-5. Expression levels of $P f d n 2$ across BXD strains and parental strains............51

Figure 3-6. Pearson correlation between Sncg and Pfdn2 expression in retinas of

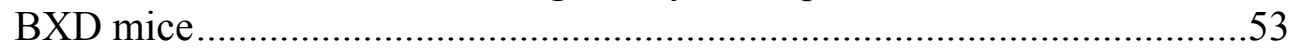

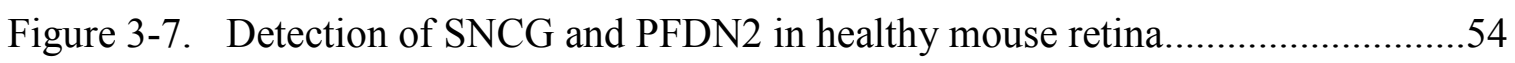

Figure 3-8. Co-localization of SNCG and PFDN2 in healthy and diseased mouse

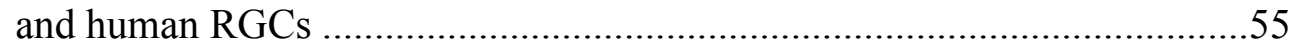

Figure 3-9. Dissociated retinal cells were labeled with Zombie Aqua ${ }^{\mathrm{TM}}$ for live/dead

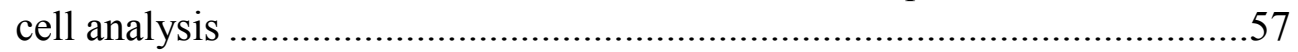

Figure 3-10. Histogram analysis showing the percentage of retinal cells labeled with anti-SNCG anti-PFDN2 antibodies ......................................................58

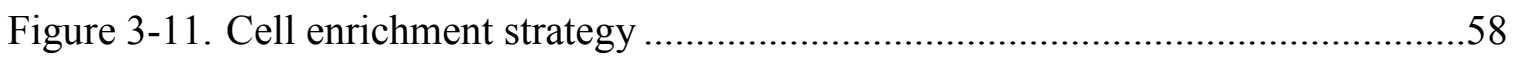

Figure 3-12. Cellular proliferation was measured by CFSE labeling.............................59

Figure 3-13. Pfdn2 knockdown inhibits Sncg expression in enriched primary RGCs ......61

Figure 3-14. Venn diagram summarizing number of gene transcripts with expression levels that are correlated with $S n c g$ and $P f d n 2$ expression levels, along with the number of shared correlates between the two groups

Figure 3-15. Gene ontology analysis of the shared correlates of Sncg and Pfdn2 are presented

Figure 3-16. Gene ontology analysis of the shared correlates of Sncg and Pfdn2 ..........65

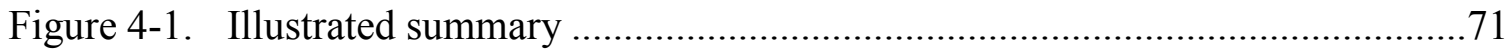

Figure 4-2. Thy $1^{+} \mathrm{CD} 48^{\text {neg }}$ surface phenotype is not sufficient to identify murine

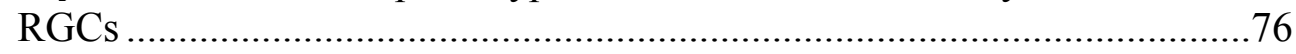

Figure 4-3. Lack of specificity of current RGC isolation methods .............................76

Figure 4-4. Validation of our improved protocol for the enrichment of RGCs...............78

Figure 4-5. Identification of Thy $1.2^{+} \mathrm{CD} 48^{\text {neg }}$ cells using qualitative real time PCR analysis

Figure 4-6. Immunohistochemical localization of surface antigens used in our RGC sorting protocol 
Figure 4-7. Immunohistochemical localization of intracellular RGC markers used as part of the validation process of our sorting protocol

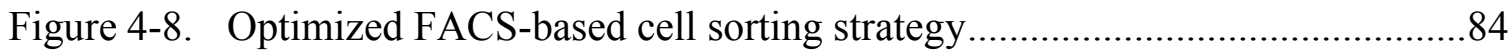

Figure 4-9. Surface marker expression of pre-sorted retinal cells .................................85

Figure 4-10. Purity of post-sorted RGCs based on the surface marker and intracellular

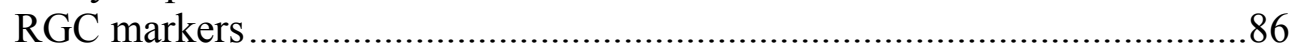

Figure 4-11. Consistency of flow cytometry results using MACSQuant ${ }^{\circledR}$.....................87

Figure 4-12. Intracellular expression of the RGC marker RBPMS in Live

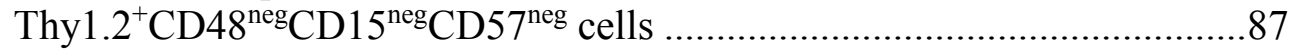

Figure 4-13. Live Thy $1.2^{+} \mathrm{CD} 48^{\text {neg }} \mathrm{CD} 15^{\text {neg }} \mathrm{CD} 57^{\text {neg }}$ show RGC morphology .................88

Figure 4-14. Validation of Live Thy $1.2^{+} \mathrm{CD} 48^{\text {neg }} \mathrm{CD} 15^{\text {neg }} \mathrm{CD} 57^{\text {neg }}$ cells using qPCR analyses

Figure 4-15. Fewer RGCs are harvested from mice with documented elevated IOP and optic nerve damage

Figure 4-16. Optic nerve damage in C57BL/6J and BXD66 young/ old mice .91

Figure 4-17. Characterization of enriched RGCs from young and old BXD66 mice obtained through our improved flow sorting method.

Figure 4-18. Localization of known intracellular RGC markers in BXD66 young/old mice

Figure 4-19. Relative purity of sorted RGCs based upon surface marker selection and intracellular RGC markers

Figure 4-20. Comparison of live retinal cells based on sorting protocol. . .95

Figure 5-1. Glaucomics 106 


\section{LIST OF ABBREVIATIONS}

$\mathrm{AF}$

$\mathrm{AH}$

ANOVA

ARPE19

ARVO

BCL2

BDNF

BMP4

BMPR1A

BrdU

BRN3A

BSA

$\mathrm{C} 1 \mathrm{q}$

C3

C4

CACNA2D1

CAV1/CAV2

CCB

CD11b

CD133

CD15

CD24
Alexa fluor

Aqueous humor

Analysis of variance

Arising retinal pigment epithelia 19

Association of research in vision and ophthalmology

B-cell lymphoma 2

Brain-derived neurotrophic factor

Bone morphogenetic protein 4

Bone morphogenetic protein receptor $1 \mathrm{~A}$

5-bromo-2'-deoxyuridine

Brain-specific homeobox/POU domain protein 3A

Bovine serum albumin

Complement component 1, q subcomponent

Complement component 3

Complement component 4

Calcium channel, voltage-dependent, alpha 2/delta subunit 1

Caveolin 1/2

Calcium channel blockers

Complement component 3 receptor 3 subunit

Cluster of differentiation antigen 133/ Prominin-1

Cluster of differentiation antigen 15

Cluster of differentiation antigen 24 


\begin{tabular}{|c|c|}
\hline CD31 & Cluster of differentiation antigen 31 \\
\hline CD44 & Cluster of differentiation antigen 44 \\
\hline $\mathrm{CD} 47$ & Cluster of differentiation antigen 47 \\
\hline $\mathrm{CD} 73$ & Cluster of differentiation antigen 73 \\
\hline CD94 & Cluster of differentiation antigen 94 \\
\hline CDC7 & Cell division cycle 7-related protein kinase \\
\hline CDKN2B-AS1 & Cyclin-dependent kinase inhibitor 2b-antisense RNA 1 \\
\hline cDNA & Complementary DNA \\
\hline CFSE & Carboxyfluorescein succinimidyl ester \\
\hline $\mathrm{Chr}$ & Chromosome \\
\hline CHX10 & Ceh-10 homeodomain-containing homolog \\
\hline CNS & Central nervous system \\
\hline CNTF & Ciliary neurotrophic factor \\
\hline $\mathrm{C}_{\mathrm{t}}$ & Comparative threshold \\
\hline DAG & Directed acyclic graph \\
\hline DNA & Deoxyribonucleic acid \\
\hline dNTPs & Deoxynucleotide \\
\hline EBAA & Eye bank association of america's \\
\hline EDTA & Ethylenediaminetetraacetic acid \\
\hline eQTL & Expression quantitative loci \\
\hline ER & Endoplasmic reticulum \\
\hline FACS & Fluorescence-activated cell sorting \\
\hline FBS & Fetal bovine serum \\
\hline
\end{tabular}




\begin{tabular}{|c|c|}
\hline FcyRII/III & $\mathrm{Fc}$ fragment of IgG, low affinity $\mathrm{IIb} / \mathrm{III}$ \\
\hline FG & Fluoro-Gold \\
\hline FITC & Fluorescein isothiocyanate \\
\hline Fmn2 & Formin 2 \\
\hline FNDC3B & Fibronectin type III domain containing 3B \\
\hline FSC & Forward scatter \\
\hline GABAb & Gamma-aminobutyric acid B \\
\hline GAS7 & Growth arrest-specific 7 \\
\hline GCL & Ganglion cell layer \\
\hline GEO & Gene expression omnibus \\
\hline GFP & Green fluorescent protein \\
\hline GLAUGEN & Glaucoma gene environment initiative \\
\hline GO & Gene ontology \\
\hline GOTM & Gene ontology tree machine \\
\hline GPNMB & Glycoprotein (Transmembrane) Nmb \\
\hline GWAS & Genome-wide association studies \\
\hline $\mathrm{h}_{2}$ & Heritability \\
\hline HBSS & Hank's balanced salt solution \\
\hline HEI & Hamilton eye institute \\
\hline HIV & Human immunodeficiency virus \\
\hline HLA-DR & Human leukocyte antigen - antigen D related \\
\hline HNK-1 & Human natural killer-1 \\
\hline $\operatorname{IgG}$ & Immunoglobulin $\mathrm{G}$ \\
\hline
\end{tabular}




\begin{tabular}{|c|c|}
\hline $\mathrm{IHC}$ & Immunohistochemistry \\
\hline IL-10 & Interleukin 10 \\
\hline IL-1B & Interleukin 1B \\
\hline IL-6 & Interleukin 6 \\
\hline IL-8 & Interleukin 8 \\
\hline ILMN & Illumina \\
\hline IOP & Intraocular pressure \\
\hline IPL & Inner plexiform layer \\
\hline IRB & Institutional review board \\
\hline IU & International units \\
\hline JNK & C-Jun N-terminal kinase 1 \\
\hline LOD & Logarithm of the odds \\
\hline LRS & Likelihood ratio statistic \\
\hline MFI & Mean fluorescence intensity \\
\hline MMP & Mitochondrial membrane potential \\
\hline mRNA & Messenger RNA \\
\hline MYCO & Myocilin \\
\hline NAC & $\mathrm{N}$-acetyl-L-cysteine \\
\hline NADH & Nicotinamide adenine dinucleotide \\
\hline $\mathrm{N}-\mathrm{CAM}$ & Neural cell adhesion molecule \\
\hline NCBI & National center for biotechnology information \\
\hline ncRNA & Non-coding RNA \\
\hline NEIGHBOR & NEI Glaucoma Human genetics collaBORation \\
\hline
\end{tabular}




$\begin{array}{ll}\text { NFL } & \text { Nerve fiber layer } \\ \text { NMDA } & \text { N-methyl-D-aspartate } \\ \text { NMDAR } & \text { N-methyl-D-aspartate receptor } \\ \text { NTG } & \text { Normal tension glaucoma } \\ \text { OMIM } & \text { Online Mendelian Inheritance in Man } \\ \text { ONH } & \text { Optic nerve head } \\ \text { ONL } & \text { Outer nuclear layer } \\ \text { OPL } & \text { Outer plexiform layer } \\ \text { OPTN } & \text { Optineurin } \\ \text { OR } & \text { Odds ratio } \\ \text { PBS } & \text { Phosphate-buffered saline } \\ \text { PE } & \text { Phycoerythrin } \\ \text { PerCP } & \text { Peridinin Chlorophyll } \\ \text { PFDN2 } & \text { Prefoldin 2 } \\ \text { PI } & \text { Propidium iodide } \\ \text { PKC } & \text { Protein kinase C } \\ \text { POAG } & \text { Primary open angle glaucoma } \\ \text { PPD } & \text { Quantitative polymerase chain reaction } \\ \text { RPCR } & \text { QTL rich region }\end{array}$




\begin{tabular}{|c|c|}
\hline RGC & RGCs \\
\hline RGC-5 & RGC 5 \\
\hline RGS7 & Regulator of g-protein signaling 7 \\
\hline RI & Recombinant inbred \\
\hline RNA & Ribonucleic acid \\
\hline RPE & Retinal pigment epithelium \\
\hline RPE65 & Retinal pigment epithelium 65 \\
\hline RPM & Revolutions per minute \\
\hline RT & Room temperature \\
\hline SAS & Statistical analysis software \\
\hline SDP & Strain distribution pattern \\
\hline SEM & Standard error of mean \\
\hline SEMA3E & Semaphorin $3 E$ \\
\hline siRNA & Small interfering RNA \\
\hline SIX6 & SIX Homeobox 6 \\
\hline SNCG & Gamma-synuclein \\
\hline SNP & Single nucleotide polymorphism \\
\hline SPF & Specific-pathogen free \\
\hline SRBD1 & S1 RNA binding domain 1 \\
\hline SSC & Side scatter \\
\hline TE & Tris EDTA \\
\hline TGF- $\beta$ & Transforming growth factor beta 1 \\
\hline THY1 & Thy- 1 cell surface antigen \\
\hline
\end{tabular}


TM

TMCO1

tRNA

TRP

TUJ1

TYRP1

UCSC

UNG

UTHSC

Ve

$\mathrm{Vg}$

$\mathrm{Vp}$

WDR36
Trabecular meshwork

Transmembrane and coiled-coil domains 1

Transfer RNA

Transient receptor potential cation channels

Neuron-specific class III $\beta$-tubulin

Tyrosinase-related protein 1

University of california santa cruz

Uracil N-glycosylase

University of tennessee health science center

Environmental variation

Genetic variation

Phenotypic variation

WD repeat domain 36 


\section{CHAPTER 1. INTRODUCTION}

\section{Primary Open Angle Glaucoma}

Glaucoma is a group of disorders that have in common a distinct type of optic nerve damage. The disease process is insidious such that central vision is usually not lost until the disease is advanced and thus many cases are left undiagnosed and untreated (1). For this reason, and others discussed below, glaucoma is a leading cause of blindness worldwide. The most frequently diagnosed types are primary open-angle, angle-closure and normal-tension, or low-tension, glaucoma.

Primary open angle glaucoma (POAG; OMIM 137760) is the most common form of glaucoma. It is a progressive optic neuropathy with characteristic changes to the optic nerve head and corresponding visual field defects $(2,3)$. Glaucoma affects $2 \%$ of individuals of European descent (4)and up to $10 \%$ of individuals of sub-Saharan African descent over 50 years of age $(5,6)$. The well-recognized risk factors associated with POAG include older age, elevated intraocular pressure (IOP), ethnicity, family history of POAG, myopia, and low diastolic perfusion pressure (7).

The main clinical features of POAG are an open iridocorneal angle and cupping of the optic-nerve head (or optic disk), as well as optic disc hemorrhages and parapapillary atrophy with corresponding loss of visual field $(8,9)$. From a pathobiological point of view, POAG is characterized by progressive retinal ganglion cell (RGC) death. RGC loss causes progressive deterioration of visual fields, leading to peripheral loss of vision. It is well known that elevated IOP plays a major role in RGC apoptosis and that reduction of elevated IOP often helps in slowing down the progression of degenerative changes in glaucoma. However only one-third to half of all glaucoma patients have elevated IOP at the initial stages (10-12). About $30-40 \%$ of patients with glaucomatous visual field defects are being diagnosed as having normal tension glaucoma (NTG) (13). Therefore, elevated IOP is now believed to be an important but not the only factor responsible for optic nerve damage. Text after this run-in head starts here and is styled Normal.

\section{IOP as a major risk factor}

IOP is a significant factor in the diagnosis and management of glaucoma. It is the only known risk factor amenable to modification. It can be argued that elevated IOP is a major risk factor and is also considered to be a causative factor in glaucoma (14). There is a clear physiological association between high IOP and glaucoma. IOP can result from ocular trauma, inflammation, dysgenesis or other forms of obstruction of the aqueous humor drainage system in the trabecular meshwork of the iridocorneal angle by iris tissue, pigment or other deposits. Clinically, IOP is closely associated with visual field deterioration and optic nerve damage. It is estimated that the heritability for adult IOP range from 0.29 to $0.62(15,16)$. Linkage studies have shown two regions to be robustly 
associated with IOP, one at 10q22 from a Tasmanian glaucoma pedigree, this region contains $B M P R 1 A$, a bone morphogenic protein receptor-interesting because Bmp 4 has been implicated in increased IOP in mice (17). And another at 5q22 region which indicates WDR36 gene which codes for WD Repeat Domain 36 in a West African cohort $(18,19)$. A recent genome-wide association study (GWAS) for IOP also found associated loci, one of which (TMCO1 gene - transmembrane and coiled-coil domains 1) overlaps with a previously published POAG GWAS locus (20). Many large-scale multicenter clinical trials, including the Ocular Hypertension Treatment Study, the Early Manifest Glaucoma Trial, and the Advanced Glaucoma Intervention Study have shown that reduction in IOP effectively delays or prevents glaucomatous visual damage $(21,22)$. Therefore, lowering of IOP currently remains the only therapeutic option for mitigating the visual deficits associated with POAG. All current treatments of primary open-angle glaucoma are aimed at reducing IOP by medical or surgical means $(23,24)$.

\section{Role of RGCs in POAG}

The cell bodies of RGCs constitute the innermost layer of the retina; they are the final output neurons of the retina. These neurons are fundamentally different from other retinal neurons because they receive input from the photoreceptors via bipolar and amacrine cells and have axons which exit the eye at the optic nerve head $(\mathrm{ONH})$. The optic nerve collects all the axons of the ganglion cells and this bundle of more than a million fibers (in humans) then passes information to the brain.

In POAG, the RGC cell bodies and axons die, which leads to cupping of the optic nerve head. The mechanism of RGC damage is complex. The damage can occur due to many complementary mechanisms. Mitochondrial dysfunction has been proposed to play a key role in RGC loss in glaucoma (Kong et al., 2009; Lee et al., 2010; Osborne, 2010) it is the center stage for interactions between anti- and pro-apoptotic Bcl-2 family members (25). Excitotoxic injury has been also proposed to be involved in RGC cell death $(26,27)$. Most current hypothesis is that, excess glutamate binds to cell surface ionotropic glutamate receptors, primarily $N$-Methyl-D-Aspartate (NMDA) receptors (NMDAR), triggering massive $\mathrm{Ca}^{2+}$ influx and activation of pro-apoptotic signaling cascades in neurons leading to cell death $(28,29)$. Other studies show that RGCs die by apoptosis in models of acute optic nerve lesion (axotomy and crush) and experimental models of glaucoma $(30,31)$. Intrensic apoptotic pathways such as $\mathrm{JNK} / \mathrm{c}$-jun and $\mathrm{Bcl}-2$ gene family are known to play a major role in $\mathrm{RGC}$ cell death $(32,33)$. In addition, oxidative damage occurs in experimental models of optic nerve injury and in human glaucoma. For example, DNA damage as well as protein and lipid peroxidation products accumulate in the TM and retina from experimental animals with ocular hypertension and in POAG patients (34-36). The complement cascade is an important component of the innate immune system to recognize and eliminate pathogens and foreign material. A study showed that $\mathrm{C} 1 \mathrm{q}, \mathrm{C} 3$ or $\mathrm{C} 4$ undergo early upregulation in rat and primate glaucoma models, DBA/2J mice, and human glaucoma (37-39). Interestingly, reactive glial cells have emerged as mediators of RGC death in different injury modalities including ocular hypertension. Glial cells exacerbate excitotoxic damage, produce pro-inflammatory and 
neurotoxic factors, and undergo detrimental changes that affects RGC viability. Although there have been substantial advances in our knowledge towards cellular mechanisms of how RGCs die in glaucoma, substantial hurdles remain to translate this knowledge to clinical practice for neuroprotection (40).

\section{Genetics of POAG}

POAG is a genetically complex group of diseases, whose familial nature of POAG has been recognized for decades. POAG is the main type of glaucoma in most populations; and more than 20 genetic loci for POAG have been reported. About $5 \%$ of (POAG) is currently attributed to single-gene or Mendelian-recessive traits (usually early-onset forms of the disease), or may exhibit a heritable susceptibility consistent with complex trait inheritance (typically adult-onset forms of the disease).

Our modern understanding of the genetic architecture of POAG has evolved since the first gene for POAG, myocilin (MYOC), was mapped and identified. Since then, more than 20 genetic loci for POAG have been reported (41). The best known and studied loci are GLC1A on chromosome region 1q21-q24 spanning the MYOC gene (42), the GLC1E locus (region 10p14-p15) with the Optineurin (OPTN) gene (43), and locus GLC1G (region 5q22.1) containing the gene WDR36 (18). Other known genes with significant association with POAG/IOP include; TMCO1 (20), antisense noncoding RNA in the INK4 Locus (CDKN2B-AS1) (44), Growth Arrest-Specific 7 (GAS7) (20), Caveolin 1(CAV1/CAV2) (45), and SIX Homeobox 6 (SIX1/SIX6) (44). However, only a small number of patients with POAG have mutations in these genes. Currently, the genetic origins of the majority of glaucoma cases are unknown, as the known genes account for only a small fraction of heritable cases. The identification of genes (and their protein products) that can cause or contribute to glaucoma will help define the underlying pathophysiology as well as lead to the development of new DNA-based diagnostic tests and novel therapeutic approaches.

\section{Candidate gene studies}

Family history is one of the risk factors of POAG, but the number of cases of POAG caused by single gene mutation accounts for only a small portion of all POAG cases. Numerous studies have focused on candidate gene approach based on either gene expression or the function or structure of specific proteins in multiple glaucoma-related tissues. This is simpler and cheaper than complete re-sequencing of candidate genes, and has been proposed as a powerful means to identify the common genetic variants that underlie complex diseases (46). The candidate gene approach has been successful for several inherited ocular diseases. However, most glaucoma cases involve low-penetrance risk alleles rather than mutations in known high-penetrant genes. This makes it difficult to select the variants of target genes in a large number of genetic variants. Since the fundamental pathophysiology of glaucoma is unknown, candidate gene studies will not sufficiently explore the genetic basis of the disease. Usually there is inconsistency in the 
association and replication studies due to variations in the study design, diagnosis method, sample size and ethnicity.

\section{Genome-wide studies}

GWAS approach has been performed in various common diseases and succeeded in identifying new susceptibility genetic regions including POAG. GWAS are casecontrol studies, based on a comparison of unrelated but cases and control individuals from a population using single nucleotide polymorphisms (SNPs) that segregate with the disease or trait. A simple study design in GWAS includes four steps: (a) selection of a large number of individuals with the disease or trait of interest and a suitable control group; (b) DNA isolation, genotyping, and quality control; (c) statistical tests for associations between the SNPs and the disease/trait; and (d) replication of identified associations in independent population samples or experimental examination of functional implications to verify a true association. This type of analysis is very efficient and powerful in identifying causative genes, especially for complex diseases. GWAS have greater power to detect the effects of common variants, since linkage disequilibrium are measured in tens of thousands of bases (47).

Meguro et al. reported the first genome-wide significant $\left(p=2.5 \times 10^{-9}\right.$, odds ratio $(\mathrm{OR})=2.80$ ) association for SNP rs3213787 in SRBDI in the Japanese NTG population (48). Other POAG associated gene variants that GWAS identified include SNPs near CAV1 and CAV2 in an Iceland cohort (45), the caveolin genes have been postulated to influence transforming growth factor-beta (TGF- $\beta$ ) or nitric oxide signaling pathways involved in POAG pathogenesis. The association of the TMCO1 locus with POAG has been replicated in another GWAS for a Caucasian cohort (20). The ciliary body, trabecular meshwork and retina show abundant TMCO1 expression. However, its precise role in POAG pathogenesis is unclear. A recent study by Li et al. provided strong evidence of an association at the $C D K N 2 B-A S 1$ locus (rs2157719, OR $=0.71, p=2.81 \times$ $10^{-33}$ ), and also identified SNP rs1 192415 in the CDC7-TGFBR3gene (1p22) showing significant association with POAG $\left(\mathrm{OR}=1.13, p=1.60 \times 10^{-8}\right)$ in the Asian, African and European populations, as well as SNP rs4894796 in FNDC3B (3q25.31) showing a significant association in Asians only $\left(\mathrm{OR}=0.89, p=7.93 \times 10^{-8}\right)(49)$. GWAS studies by Wiggs et al. and Osman et al. in the Caucasian POAG and Japanese POAG cases, respectively, have demonstrated a strong association of SNP rs10483727 located in the in the intergenic region between the SIX1 and SIX6 locus (14q23) (50, 51). SIX6 has been shown to express in the developing and adult human retina (52).

GWAS are advantageous since there is no need to include a specific disease model, which can allow identification areas of previously unsuspected pathogenesis. The pathogenesis of most cases of glaucoma, excluding those in which single gene defects have been identified due to contributions from many different polymorphisms. So likelihood of picking up an association depends on the ability to control for errors due to bias and poor phenotyping. GWAS need large numbers of cases and controls to identify the biological context in which statistically significant candidate variants act. This may reflect linkage disequilibrium between the SNPs identified and the true causative 
functional variant. Therefore, fine mapping of the regions harboring the most significant statistical signals is need to be followed up by GWAS. These type of studies have significant cost implications.

\section{Systems Genetics Approach to Study Glaucoma}

Systems genetics is an approach to understand molecular architecture that underlies complex traits. The multiple genetic and environmental factors that play a role in glaucoma make it difficult to identify individual genes linked to this disorder. Still, some genetic risk factors associated with forms of glaucoma have been identified in genes that code for proteins involved in known biological pathways. Despite this progress, determining which genes may be the most relevant to developing therapeutic interventions for glaucoma has proven exceedingly difficult. The major obstacles being that gene/disease associations reveal very little about the underlying basic molecular biology and any implicated gene variant explains only a small proportion of an individual's overall risk for the disease. Recent advances in technology has provided tools that are capable of tracking genome-wide expression changes occurring in progressive pathological processes and diseases such glaucoma. It is becoming increasingly evident that the application of network-based genetics or systems genetics approaches can provide insights into not only the roles of individual genes or developmental pathways but also illuminate relationships between different levels of a biologic system, such as the genome, transcriptome, and phenome $(53,54)$.

Systems genetics is a relatively new branch of quantitative genetics that has the goal of understanding networks of interactions across multiple levels that link DNA variation to phenotype. Systems genetics involves an analysis of sets of causal interactions among classic traits such as IOP, networks of gene variants, and developmental/environmental/epigenetic factors. The main challenge is the need for comparatively large sample size and the use of more advanced statistical and computational methods and models. Genetic reference populations, which are genetically well characterized lines, are often used in systems genetics studies as a source to study a multitude of different phenotypes and to find the causal relations from networks of genetic correlations.

\section{Recombinant inbred strains}

Recombinant inbred (RI) strains are a collection of genetic reference populations commonly used in genetic mapping of Mendelian and quantitative traits. Bailey and colleagues introduced the idea of using RI lines for detecting major-gene effects and to establish linkage (55-57). Conventionally, RI strains are developed by crossing two inbred parental strains. The inbred lines are mated repeatedly for 20 or more generations to warrant they are at least $99 \%$ inbred (55). Consequently, each RI line produced has two identical copies of each chromosome and is just a pure mosaic of the parental genomes. These homozygous lines contain the allele of either one or the other parental 
strain. For a segregating trait, the linkage can be determined by matching the allelic pattern for the locus across the RI strains to the previously known markers. This is known as the strain distribution pattern (SDP). Generally, a qualitative trait is controlled by a single major gene, in contrast complex quantitative traits are influenced by moderate effects due to several genes. With the latest technologies, there has been a tremendous improvement in genotyping of RI strains with new markers and increasing data on SDP. RI strains are also used in identifying quantitative trait loci (QTLs) associated with quantitative traits (58-60). This idea was first introduced in late 1970s by correlating genetic marker information with quantitative phenotypes $(61,62)$. Classically, the Pearson correlation was used to measure association and the strength of this association was improved by using multiple markers and an interval mapping approach (63). The effectiveness of the RI strains depends on the size of the panel; the big panels tend to map the trait precisely. These strains are particularly useful for precise estimation of phenotypes associated with low to medium heritability.

\section{$D B A / 2 J$ inbred strain as glaucoma mouse model}

The development of new therapeutic drug targets for elevated IOP relies heavily on animal models, which represent a valuable tool for understanding both the progression and cause of glaucoma. The damage present at the time of diagnosis precludes the study of disease development from onset. Additionally, obtaining retinas at different pathologic states is difficult and is a limitation. Much of the progress in the study of glaucoma has been driven by the development of rat models is due to similar ocular anatomy to humans. They are readily available, easy to genetically manipulate and have a short life span. All these reasons make rodents a very attractive models to use as research tools for studying glaucoma. However, studies on mice have a distinct advantage over rats because these animals enable us to conduct complex genetic manipulations in order to study the function of crucial genes and pathways in glaucoma.

John et al developed DBA/2J as a mouse model of glaucoma that has become well established over the past few years (64). DBA2J mice develop elevated IOP and severe glaucoma like phenotype with age, followed by RGC death, and optic nerve degeneration. The IOP elevation is subsequent to an iris disease that deposits pigment in the ocular drainage structures. The iris transillumination defect is caused by mutations in two different genes- Tyrp 1 and Gpnmb. The first is the mutation in the b allele of tyrosine related protein $\left(\right.$ Tyrp $\left.^{b}\right)$. Tyrpl encodes a melanosomal protein that plays a role in enzymatic and structural functions in these organelles. The second mutant gene is Gpnmb which encodes a transmembrane glycoprotein found in multiple different cellular structures. The Gpnmb gene carries a stop codon mutation $\left(G p n m b^{R 150 X}\right)$. These mutations together lead to anterior chamber changes including pigment dispersion, iris transillumination, iris atrophy, anterior synechia, and elevated IOP.

A significant advantage in using DBA2J mice include the inbred nature of these mice, meaning that they have a fixed genetic background. Despite this, these mice exhibit a high degree of variability and asymmetry in developing the disease (65).

Approximately $8-10 \%$ of the older DBA/2J mice have a combination of one normal, non- 
diseased retina and optic nerve, and one eye undergoing complete degeneration. Also, the relevance of TYRP1 and GPNMB to human glaucoma is controversial since no exonic mutations in either gene have been found in human glaucoma patients $(66,67)$. The unusual interplay of the Trypl and Gpnmb genes in initiating the anterior chamber disease is not related to POAG in humans.

\section{BXD strains}

The largest and best-characterized murine reference panel is the BXD family of RI mouse lines (68) (Figure 1-1). The first of BXD lines from 1-42 were produce by researchers at Jackson laboratory and lines 43-100 were produced at University of Tennessee Health Science Center led by Dr. Robert Williams. The new set of BXD strains from BXD 43-108 were produced from advanced intercrosses, which makes them highly recombinant. These advanced intercross lines contain twice as many recombinations as the older BXD strains. The complete genomic sequence data of both the parents are publicly available (www.genenetwork.org).

The BXD strains are well suited for integrating data across multiple phenotype domains spanning molecular, morphological, physiological, and behavioral traits. BXDs have been used extensively in genetic and genomic studies of the eye and central visual system (69-71). The DBA/2J strain of mice is one of the parental strains of the BXD family. It closely mimics human hereditary pigmentary dispersion glaucoma. The greatest utility of this BXD family is that it can be used to map the chromosomal positions of sequence variants that cause differences in gene expression. Furthermore, reproducibility of eQTLs in the BXD family and in an F2 cross between the same parental strains has been reported to be very high (72).. Over the last two decades, the BXD family has been exploited to study the genetics of immune function and infectious disease $(73,74)$. It has also been used in behavioral and neuro-pharmacological research $(75,76)$. The eyes and retinas of this BXD family have also been well studied for more than a decade, and we now possess extensive cytological and morphometric data on their eyes and retinas that can be studied with reference to differences in gene expression. Due to the fixed genotypes of each BXD strain and the availability of massive databases of expression data it is possible to study correlations between a phenotype and genotypes. We can also study associations between two or more genes and identify upstream genetic regulators.

\section{Genetic dissections of genomic data}

\section{QTL analysis}

A forward genetics approach that is frequently used for studying complex traits in animal models is called QTL mapping. QTL analysis is a statistical method used to measure a particular quantitative trait across a genetically diverse population and screening for associations between genotypic and phenotypic variation $(77,78)$. The main purpose is to detect the polymorphisms underlying the phenotype of interest by scanning for alleles that co-vary with the traits. The regions of the genome that show a 


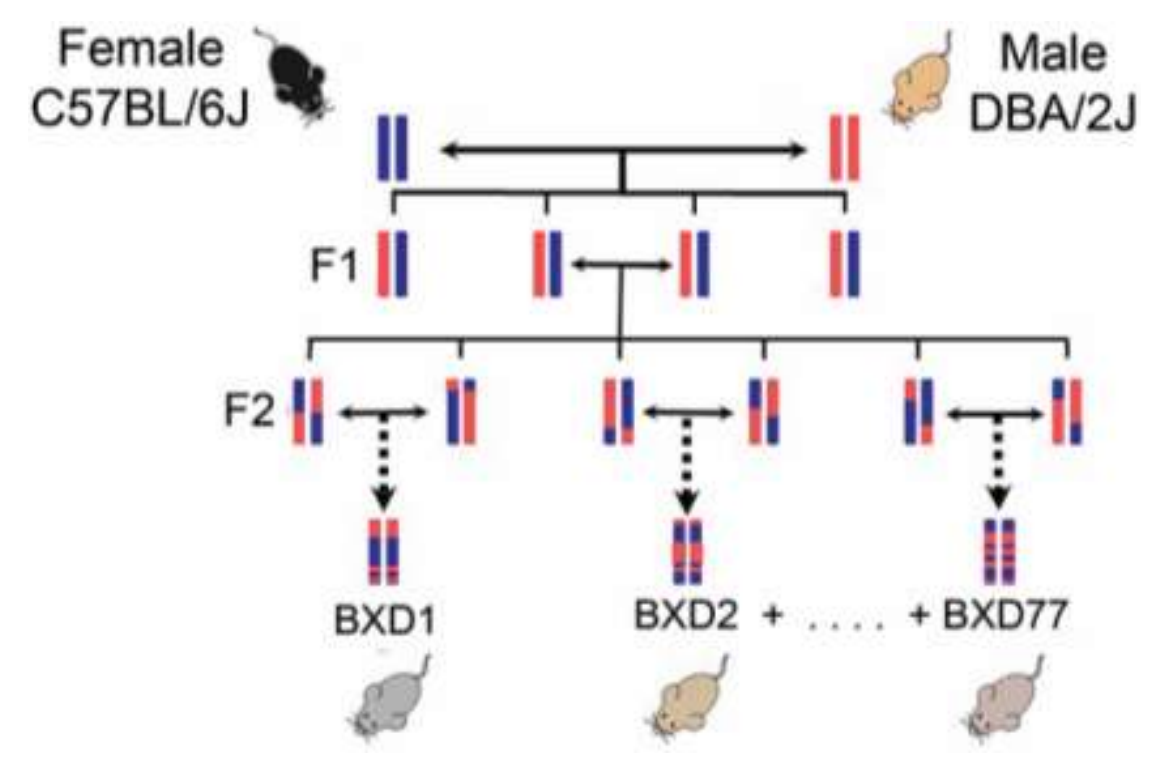

Figure 1-1. BXD breeding scheme

All BXD lines were derived by crossing C57BL/6J and DBA/2J parents. The resulting heterozygous F1 individuals were again crossed to generate genetically diverse but nonreproducible F2 animals. These F2 progeny were iteratively inbred until generation F20+, at which point each line represents a unique mosaic of B6 and D2 alleles. 
significant association with a phenotype are considered QTL. In short, the mapped QTL is the implicated region harboring a single gene that affects an associated phenotype. However, it is possible that a QTL may harbor or be controlled by multiple genes, noncoding RNA (ncRNA) species, epigenetic mechanisms, or in combinations. Markers like SNPs and microsatellites are used for genotypic distinctions (79). Many QTL mapping studies have been carried out in human populations, but due to the excessive non-genetic variance contributed by each subject's unique environment and life experiences, it presents a tremendous amount of noise (80). In contrast, mice are a highly attractive model for the purpose of dissecting complex traits. Mice are small in size, they have relatively low maintenance costs and short gestation period. With increasing advancement in technology there is an ever-expanding arsenal of genetic tools. QTL mapping studies are primarily conducted using inbred strains of mice and their various inbred lines (81).

The following are the four steps to achieve QTL mapping; the first step is to measure variation in a quantitative trait in a genetic reference population such as inbred mouse strains. The prerequisite is to have the quantitative traits that show phenotypic variability among the inbred strains. The phenotypic variation $\left(V_{P}\right)$ is due to the genetic $\left(\mathrm{V}_{\mathrm{G}}\right)$, environmental factors $\left(\mathrm{V}_{\mathrm{E}}\right)$ and interactions between them. The higher the variation due to genetic factors, the better is the mapping resolution of QTLs. The second step is to determine the heritability of the observed phenotypic variation. Heritability $\left(\mathrm{H}_{2}\right)$ you an estimation of the genetic contribution to variation in phenotypic trait. It is calculated as the ratio of phenotypic variance that is due to genetic factors to the total variance (Equation 1.1).

$$
\mathrm{H}_{2}=\mathrm{V}_{\mathrm{G}} / \mathrm{V}_{\mathrm{P}} \text { where } \mathrm{V}_{\mathrm{P}}=\mathrm{V}_{\mathrm{G}}+\mathrm{V}_{\mathrm{E}}
$$

The third step is to proficiently collect the phenotype and genotype data from the inbred strains. The phenotype is any measurable or quantitative trait and genotype are various markers such as SNPs or copy number variants (CNVs). Such genotype markers are unique for individual strains and follow a particular strain distribution pattern. The fourth step is to generate a linkage map and perform the statistics generating a QTL. The most commonly used methods for doing a linkage analysis are- regression method, likelihood method, variance component method and the Bayesian method. For our study, we use GeneNetwork (http://www.genenetwork.org/webqtl/main.py), which utilizes interval mapping to estimate the position of QTLs across a chromosome $(60,71,76,82)$. The significant linkage between the phenotypic trait and genotype is generated by using permutation test and by calculation of the likelihood ratio statistic (LRS).

\section{Expression QTL}

Technological advancement has enabled us to use systems-level approach in identifying disease-associated genes using gene mapping methods and high throughput molecular data. QTL mapping methods were previously used for classical physiological or behavioral phenotypes, but now we can identify QTLs for genetic factors influencing high-throughput measures of transcript, protein and metabolite. This approach of 
mapping effects of genetic variation on gene expression has been utilized in numerous studies in humans $(83-85)$, mice $(82,86)$ and other organisms. Collectively, the regions of genome (loci) in which gene expression level is influenced by specific genetic variation are termed expression quantitative trait loci (eQTL). eQTL analysis provides an understanding of how changes in the genome affect transcript expression levels, providing further insight into how transcripts regulate disease. Typically, in eQTL mapping, regulatory variants have been commonly divided into cis or trans acting QTLs depending on their location and mechanism by which they affect gene expression. In the classical definition, cis-eQTLs refers to genetic variants that affect expression of a locus on the same DNA molecule, thus in allele-specific fashion. In contrast trans-eQTLs are expected to affect gene expression in an allele independent manner. Much of the variation at the genomic level that affects a trait occurs by cis-acting sequence differences. TranseQTLs usually have smaller effect sizes and seem to be more tissue-specific than ciseQTLs $(86,87)$, thus complicating their detection.

\section{Application of Flow Cytometry in Eye}

Flow cytometry is an analytical method that measures optical and fluorescence characteristics of single cells (or any other particle, including nuclei, microorganisms, chromosome preparations, and latex beads). Flow cytometry is rapid, reproducible, and sensitive, and can be applied to living cells in suspension, making it especially an attractive method in the study of cytological specimens. The ability to simultaneously measure multiple parameters on a cell-by-cell basis is perhaps the most powerful aspect of analytical flow cytometry. Characteristics that can be measured include cell size, cytoplasmic complexity, DNA or RNA content, and a wide range of membrane-bound and intracellular proteins. Cellular and physical properties, such as size or surface area (represented by forward angle light scatter) and internal complexity/granularity (represented by right-angle scatter) can resolve certain cell populations. The methodology relies on the use of antibodies conjugated to different fluorochromes, each with similar excitation wavelengths and different emission wavelengths (or "colors"). This allows several cellular properties to be measured simultaneously based on the mechanism of action of the fluorochromes associated with a dye, the specificity and sensitivity(88). Certain dyes such as fluorescein isothiocyanate [FITC], propidium iodide [PI], bind to specific proteins/lipids/nucleic acids to increase fluorescence. The fluorescence intensity may vary based on cellular physiological parameters such as $\mathrm{pH}$, membrane potential, as well as enzymatic activity (fluorogenic substrates) such as esterases, peroxidases, and peptidases.

\section{Instrumentation}

Flow cytometers comprise three main systems: fluidics, optics and electronics. Inside a flow cytometer, cells in suspension are drawn into a stream created by a surrounding sheath of isotonic fluid that creates laminar flow, allowing the cells to pass individually through a sensing point which is called the hydrofocus point. At the sensing 
point, a beam of monochromatic light, usually from a laser, intersects the cells and produces the fluorescence emission. Emitted light is scattered in different directions and is collected via optics that directs the light to a series of filters and dichroic mirrors that isolates particular wavelength bands. The light signals are detected by photomultiplier tubes and converted into electrical signals by the optical and electronics system. The electric signals are converted into numeric data by different algorithms contained in the data acquisition component. Resulting data is analyzed using as additional software and represented in either single or two dimensional plots.

\section{Flow cytometry application in ocular diseases and retinal cell biology}

A growing body of literature supports the application of flow cytometry in many fields of research such as immunology, cancer, cell biology, stem cell and biomolecular screening (89-91). Its application is not limited to just basic research where they are used for performing tasks that require analytical precision and high throughput. Flow cytometry instrumentation plays a key role in hospital and medical centers, where they are widely used for clinical diagnosis. However, in spite of the breadth of applications straddling many fields, the use of flow cytometry in neuroscience has been largely unexploited; being perceived as challenging and has seen only a steady increase in interest until recent years. Over the last decade, flow cytometry has proved useful in studying neuronal population in brain, CNS and eye $(92,93)$. Increased awareness of the quantitative and overall analytic potential of flow cytometry has recently encouraged its more widespread use in ophthalmology.

The analysis of retinal cells by flow cytometry in basic and clinical vision research is becoming more common in recent years. Cousins et al. identified and analyzed antigen-activated lymphocytes and their role of infiltrating immune effectors in ocular inflammatory disease using flow cytometry. They distinguished activated $\mathrm{T}$ and $\mathrm{B}$ lymphocytes from mouse eyes (94). Flow cytometry was also used later by Davis et al. to diagnose intraocular lymphoma and ocular inflammatory disease using patient vitreous specimens (95). Researchers have also utilized this technique to investigate HLA-DR on conjunctival epithelial cells from patients with cystic fibrosis using monoclonal antibodies to HLA-DR (96). Another example is in dry eye disease where Chen et al. used this analytical method to delineate the $\mathrm{CD} 4^{+} \mathrm{T}$-cell populations in normal, acute DED, and chronic DED mice (97). Flow cytometry of vitreous fluid samples has proven useful in the diagnosis of intraocular lymphoma, a subset of primary CNS lymphoma (98). A retrospective study of 20 patients with suspected intraocular lymphoma on slit lamp examination, 7 of 10 patients diagnosed with intraocular lymphoma by vitrectomy had positive flow cytometry findings (99). Flow cytometry had higher positive and negative predictive values compared with cytology. Several other retinoblastoma studies have looked at cell cycle regulation, proliferation and apoptosis using flow cytometry. $(100-102)$

Many laboratories have used standard intracellular or surface markers to identify neuronal cell types in the retina. In 2011, Samuel et al. used antibodies against recoverin 
to detect photoreceptors, syntaxin for amacrine, $\mathrm{Chx} 10$ for bipolar, $\mathrm{PKC}$ for rod bipolars, Thy1 for RGCs (103). They were able to identify changes the surface marker levels in these cell types due to age related changes. A similar study by Portillo et al. identified microglia/macrophages, endothelial cells, astrocytes, photoreceptors, ganglion neurons and Müller cells using CD11b, CD31, glial fibrillary acidic protein, rhodopsin, Thy1 and vimentin, respectively in mouse retinas (104). Apart from identifying subtypes of cells, one can also assess viability and damage in neuronal cells. Chang et al., measured viability and damage using BrdU, in small, medium and large RGCs in rats after eye injury by N-methyl-D- aspartic acid (NMDA)-injury. They identified the RGCs using Fluoro-Gold (FG) and were able to determine the size of the RGCs using flow cytometry. The damage was assessed using FG vs Thy1 intensity (105). The most common application of flow cytometry is assessment of toxicity, apoptosis and oxidative stress.

Another utility of FACS is the ability to quantify specific neuronal populations. Few successful attempts have been made in past, Kennedy et al. performed a simple technique to quantify rod outer segment phagocytosis in cultured RPE cells using FITC labeled rod outer segment to tag rod photoreceptors (106). Srinivastava et al. analyzed the RPE cell line ARPE19, human skin fibroblasts and human corneal epithelial cells by determining RPE65 protein expression and epithelial and fibroblast proteins (107). By focusing on identification of novel surface markers we can isolate subpopulations of retinal cells using Fluorescence-activated cell sorting (FACS). FACS of live cells separates a population of cells into sub-populations based on fluorescent labeling. Using FACS, Xu et al., isolated $\mathrm{CD} 133^{+} \mathrm{CD} 44^{+}$retinal cells to study cell type composition of retinoblastoma tumors in mouse (108). Similarly, Feodorova et al., used size based inclusion method to isolate rod perikarya from adult mice, based on their high backscatter of heterochromatin in their nucleus (109). Lakowaski et al., used combination of five cell surface markers to facilitate the isolation of photoreceptor precursors, with a phenotype CD73(+)CD24(+)CD133(+)CD47(+)CD15(-) from three-dimensional model of retinal differentiation from mouse embryonic stem cells (110). Apart from these, flow cytometry and FACS can be utilized in many other domains and new applications have been developed in ophthalmology, for investigating ocular surface biology and pathophysiology.

Flow cytometry has also proved useful in studying glaucoma-associated changes in the eyes. Liu et al. have used flow cytometric study to assess the amount of inflammatory cells or cellular debris induced by lase iridotomy, which is a treatment method for pupillary-block glaucoma (111). A similar study by Cvenkel et al., determined the levels of IL-8, IL-1B, IL-6, IL-10 in aqueous humor of patients who underwent antiglacomatous surgery by flow cytometry (112). One of the implications of pathological changes associated with glaucoma is oxidative stress which leads to mitochondrial dysfunction. McElnea et al. examined those changes in human lamina cirbrosa cells from glaucoma donors. They evaluated mitochondrial membrane potential (MMP) and intracellular calcium $\left[\mathrm{Ca}^{+}\right]$levels by flow cytometry using the $\mathrm{JC}-1$ (5,5',6,6'-tetrachloro-1,1',3,3'-tetrabenzimidazolecarbocyanine iodide) and fluo-4/fura red probes respectively (113). John and colleagues utilized flow cytometry to demonstrate the 
natural deficiency of CD94 that underlies the DBA/2J mice, concluding it is not a pathogenic factor for glaucoma progression (114).

\section{Research Aims}

\section{Aim 1: To identify the candidate gene that modulates IOP in mouse and test translational validity of the mouse candidates using the cohorts of human glaucoma patients}

1. Which gene variants modulate IOP in BXD mice strains?

2. Do mouse candidate genes translate to human glaucoma patients?

\section{Aim 2: To identify and validate regulator of Sncg in primary mouse RGCs}

1. Can we use systems genetics to identify upstream modulator of Sncg?

2. Can we combine systems genetics with other molecular techniques to validate the candidate gene?

\section{Aim 3: To isolate and validate primary murine RGCs from healthy and recombinant inbred mouse model of elevated IOP}

1. Can we isolate primary murine RGCs using flow cytometry?

2. Can we apply this protocol to isolate RGCs from mouse model of glaucoma? 


\section{CHAPTER 2. SYSTEMS GENETICS APPROACH TOWARDS UNCOVERING THE GENE MODULATORS OF IOP}

\section{Introduction}

Our aim was to identify new candidate gene variants for high IOP and to apply systems genetics approach to identify networks of genes that are critical for IOP. In this study we have systematically measured IOP across a large subset of BXD strains at 9.113 months of age. These quantitative measurements have been deposited to an openaccess web service for systems genetics (www.genenetwork.org). We identified candidate gene variants by stringent stepwise refinement based on eQTL mapping, correlation analyses (direct and partial Pearson test) and single nucleotide polymorphisms (SNPs). We combined mouse SNPs with human GWAS glaucoma cohorts from GLAUGEN/NEIGHBOR consortium database in an effort to identify and validate any causal SNP within candidate genes. Here, we report for the first time a systems-genetics analysis of the genetic components regulating IOP (Figure 2-1).

\section{Methods}

\section{BXD strains}

IOP study: A total of 545 mice were used in this study Age 9.1-13 months (548 mice from 66 BXD strains) Appendix A, Table A-1. The gender distribution was relatively equal with 313 females and 235 males being included. All procedures involving mice were approved by the Animal Care and Use review board of UTHSC and followed the Association of Research in Vision and Ophthalmology (ARVO) Statement for the Use of Animals in Ophthalmic and Vision Research in addition to the guidelines for laboratory animal experiments (Institute of Laboratory Animal Resources, Public Health Service Policy on Humane Care and Use of Laboratory Animals). Animals were housed under cyclic light (12 hour on: 12 hour off) with 35\% humidity in a specificpathogen free (SPF) facility at UTHSC. Animals were fed a 5\% fat Agway Prolab 3000 rat and mouse chow (Breeder Chow, Teklad) and water ad libitum.

\section{IOP measurement}

IOP was measured using the induction-impact tonometer (Tonolab tonometer, Colonial Medical Supply, Franconia, NH) for rodents according to the manufacturer's recommended procedures. For measuring the IOP, the tonometer was fixed in a vertical position to a support stand by means of clamps. The mouse was gently restrained by hand on an adjustable stand, and the eye was oriented in such a way as a distance of 1-4 mm was maintained between from the tip the probe to the cornea of the eye. Six consecutive 


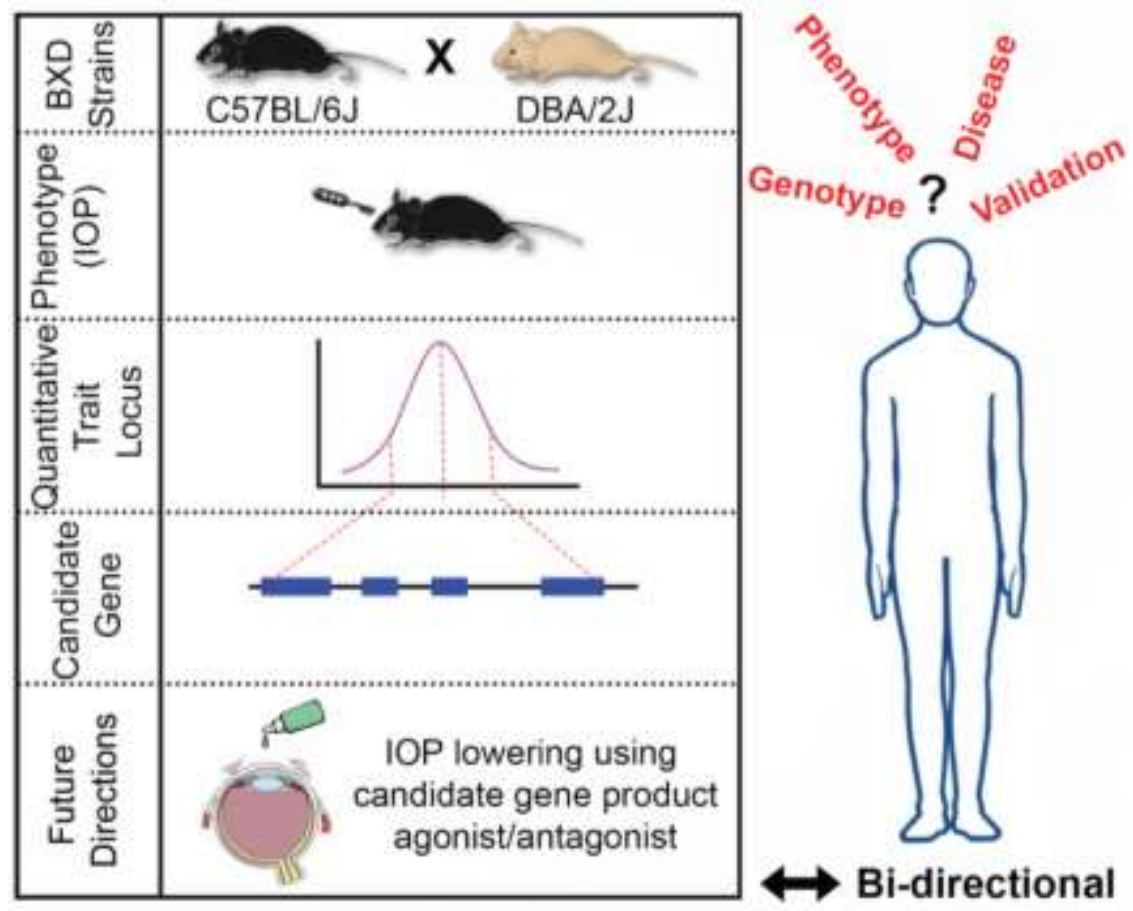

Figure 2-1. Illustrated summary

A systems genetics based study to identify and validate genetic modifiers of IOP using the enlarged BXD family of strains in combination with human GWAS glaucoma cohorts. In future, this will pave the way for improved drug development tailored to individual genotypes for POAG. 
IOP readings were averaged. IOP readings obtained with Tonolab have been shown to be accurate and reproducible in different mouse strains, including DBA/2J (115).

\section{Identification of significant genes for IOP in mouse and human}

IOP data generated from 9.1-13 months old BXD strains was integrated into the GeneNetwork database. The identification and mapping of phenotypic QTL (pQTL) was performed by linking trait data to genotypes at known genetic marker loci using the WebQTL module on GeneNetwork (http://www.genenetwork.org/). Genome wide significance levels were estimated by performing 2000 permutations based on our published methods (Lu et al., 2011a; Lu et al., 2011b; Swaminathan et al., 2013). This analyses produces a LRS score, providing us with a quantitative measure of confidence of linkage between the IOP and SNP markers. For the identification of single QTLs, interval mapping analyses were performed across all chromosomes. The parental strains were included in all mapping analysis. Composite interval mapping was performed for IOP at 9.1-13 months, choosing genetic markers with highest LRS at the single QTL region or interacting loci from pairwise interaction scans, by factoring out of the effect of the preeminent QTL the power to identify secondary QTLs on other chromosomes increased.

Human Genome-wide genotype data were obtained from the NEI Glaucoma Human Genetics Collaboration (NEIGHBOR) and the Glaucoma Genes and Environment (GLAUGEN) consortia, containing whole genome genotypes for 2170 cases and 2347 controls. 495,132 SNPs were analyzed for GLAUGEN and 523,528 SNPs for NEIGHBOR (116). Logistic regression to assess the association between individual SNPs and POAG was done using PLINK v1.07. The human GWAS significance values were Bonferroni corrected for multiple comparisons using the total number of homologous genes compared and the number of tests used.

\section{eQTL analysis of IOP candidate genes}

In this study, we aimed to infer causal mechanisms for IOP variation in BXD. Observing difference in gene expression that result from variants in the identified genomic region increases the confidence that this locus harbors causal candidates underlying the IOP peaks. Therefore, we chose to restrict our candidate search to the genes located in significant eQTL region for IOP. Candidate gene was identified using the GeneNetwork/UCSC Genome browser. Gene expression can be used as a micro trait to map regulatory eQTLs for the differences in mRNA expression levels over the panel of BXD lines since it's a heritable trait. Whole eye expression levels were used as quantitative traits and implemented into interval mapping analyses to identify regulatory loci. The HEI Mouse Eye Dataset contains the data analysis of 221 Affymetrix M430 2.0 arrays (Affymetrix, Santa Clara, CA). This database presents the whole eye transcriptome profiles of 80 strains of BXD mice aged between 2 and 4 months (117). Whole eye database was used since IOP modulation is facilitated by tissues in anterior chamber of 
the eye. eQTL for a specific transcript was denoted cis-QTL if the associated marker was localized within a $10 \mathrm{Mb}$ distance of the gene position.

\section{Immunohistochemistry}

Mouse and human donor whole eye sections $(10 \mu \mathrm{m})$ were blocked with $10 \%$ normal goat serum in PBS containing $0.25 \%$ triton $\mathrm{X}-100$ for $30 \mathrm{~min}$ at room temperature, then incubated overnight at $4^{\circ} \mathrm{C}$ with the anti-CACNA2D1 primary antibody (Bioss Antibodies, Woburn, MA) diluted in the same buffer. After three washes with PBS, slides were incubated at room temperature for 2 hours with Alexa-Fluor-488conjugated secondary antibody (Invitrogen, Waltham, MA) and TO-PRO-III iodide (1:4000; Invitrogen) as a nuclear counterstain, washed with PBS again, and mounted with Vectashield anti-fade mounting medium (Vector Laboratories, Burlingame, CA). Sections were viewed and images were obtained using a Nikon $\mathrm{C} 1$ confocal microscope (Nikon, NY) within the Imaging Core Facility in the Hamilton Eye Institute. EZ-C1 free viewer software was used for image acquisition and processing.

\section{Statistics}

To determine how much of the variation in candidate gene across the cohort was due to genetic effects, we calculated the heritability of the candidate gene using the formula of Hegmann and Possidente based on our published methods. Pearson productmoment correlations were calculated using the Correlation matrix tool available in GeneNetwork. Pearson correlation measures the strength of a linear association between two variables and is denoted by $\mathrm{r}$, which in our case is IOP $(\mathrm{mmHg})$ and Cacna $2 \mathrm{dl}$ mRNA expression. Within-strain and between-strain variances were calculated with analysis of variance (ANOVA) using SAS 6.0.

\section{Results}

\section{Study design for genetics of IOP}

We measured IOP in 66 strains of BXD including both males and females Appendix A-1, Table A-1. IOP was measured at 9.1-13 months of age. One of the advantages of measuring IOP at 9.1-13 months of age is that at this stage of life span these mice strains have already developed high IOP and the process of optic nerve damage has started. The main advantage of the genetically diverse BXD over non-inbred strains (such as an F2 intercross) is that the heritability of a trait can be accurately measured and reproduced since the strains lack heterozygosity (Figure 1-1). The differences among the isogenic strains is essentially due to genetic effects whereas differences within the strains is due to environmental effect (118). BXD strains serve as a tool for mouse genetics due to the conservation of the mammalian physiology. 


\section{BXD family has a wide variation in IOP}

Since glaucoma is a complex disease caused by the combined effect of genetic and environmental risk factors we improved the power and precision of QTL mapping studies by including large number of strains (i.e genotypes) in the genetic study of IOP using BXDs. Among strains IOP varied (Figure 2-2) with BXD38 having the lowest IOP of $9.6 \pm 0.92(\mathrm{mmHg} \pm \mathrm{SEM})$ and BXD44 having the highest at 21.82 \pm 1.67 . The average IOP among all BXDs was $15.67 \pm 0.29$. The parental lines, C57BL/6J and DBA/2J, had IOP levels of $15.22 \pm 0.77$ and $16.84 \pm 0.70$, respectively (Figure 2-2). Heritability was calculated as the ratio of the within-strain variance to total sample variance. Based on our previous published study (119), heritability $\left(\mathrm{h}^{2}\right)$ for IOP for BXDs at 9.1-13 months averaged to $\sim 0.31$. This value implies that $31 \%$ of the total trait variance is attributable to genetic factors and is comparable to that of other ocular traits to which QTL mapping has been applied successfully. The more heritable a trait, the higher the likelihood it will map to significant QTLs. Consistent with this significant heritability value, IOP becomes a particularly attractive trait for genetic dissection. Interestingly, Tyrpl and Gpnmb genotypes did not influence IOP in BXD strains at 9.1-13 months (Figure 2-3A). There was no statistically significant correlation between the IOP and those genotypes (Figure 2-3B). IOP in BXD strains at 9.1-13 months has no statistically significant sex differences and no strong correlation between males and females. They fall within $95 \%$ confidence interval (Figure 2-4). This indicates that IOP could be a phenotype where variation cannot be reliably attributed to genetic factors (i.e., variation is due to environmental or technical influence).

\section{QTL on Chr5 link causal loci for IOP}

We mapped the variation in IOP to a narrow locus at the distal tip of $\mathrm{Chr} 5$, peaking at or beyond reference SNP rs 13478120 (LRS=19.6, LOD=4.25, Figure 2-5), which corresponds to human $\mathrm{Chr} 7: 81,946,444 \mathrm{bp}$ to $82,443,798 \mathrm{bp}$. IOP does not map to either Tyrp1 or Gpnmb locus (Figure 2-6). Therefore, polymorphisms within this QTL must be modulating IOP at 9.1-13 months of age.

\section{Initial identification of candidate gene in the Chr 5 QTL region}

To determine the candidate gene variants that modulate IOP we used the following criteria:

1. Pearson correlation analysis

2. partial correlation analysis

3. location within confidence intervals of murine QTLs

4. expression in the eye

5. gene size

6. true cis modulation of candidate genes in eye and/or presence of coding differences segregating among progeny 


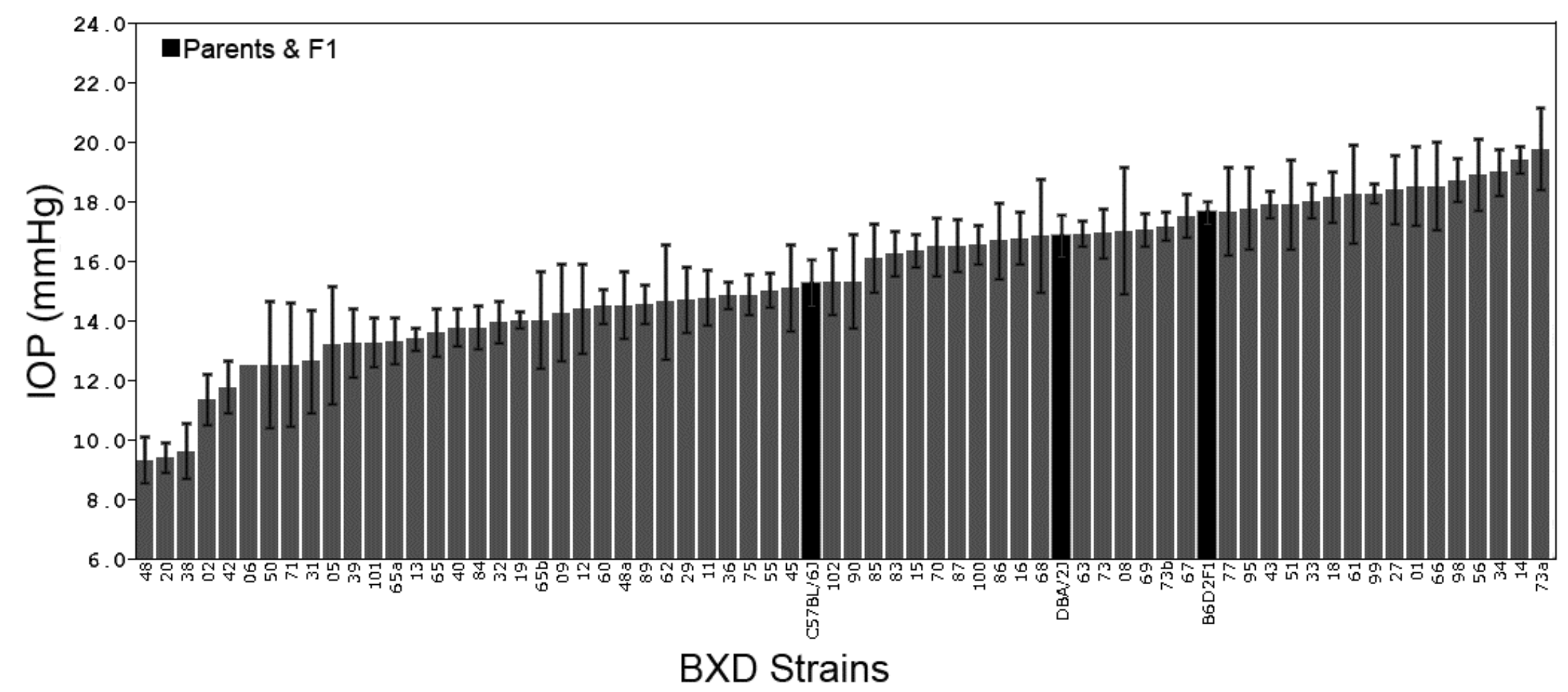

Figure 2-2. IOP levels across BXD strains and parental strains

Rank-ordered IOP levels across the BXD recombinant inbred family. BXD strains and parental strains are represented in grey and black bars, respectively. Values denote IOP levels on $\mathrm{mmHg}$ scale (mean \pm SEM). 

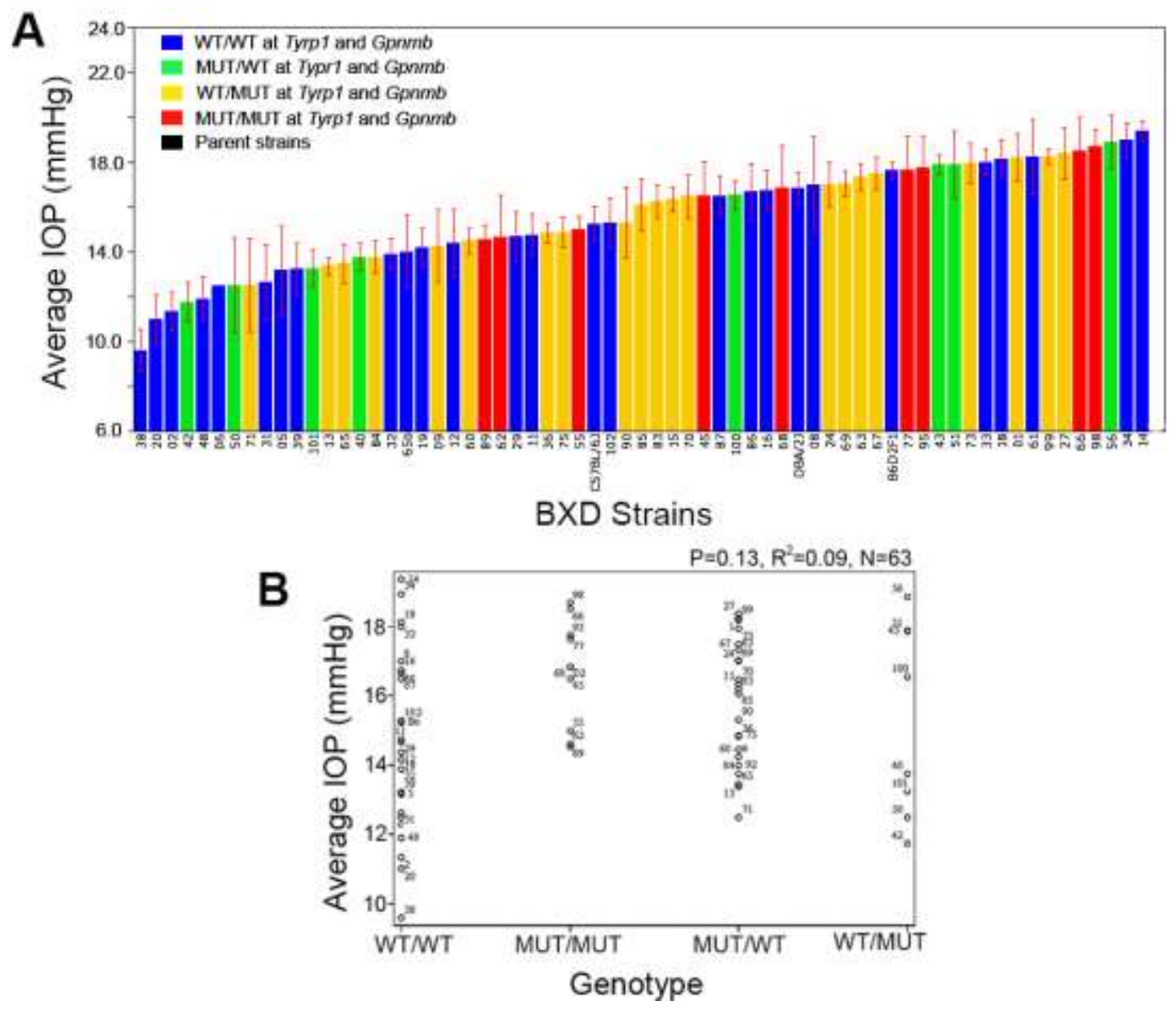

Figure 2-3. Tyrp1 and Gpnmb genotypes do not influence IOP

(A) BXD strains carrying wild type alleles of both Tyrpl and Gpnmb (WT/WT; Blue bar), mutant of Tyrpl and wild type allele of Gpnmb (MUT/WT; green bar)., wild type of Tyrp1 and mutant allele of Gpnmb (WT/MUT; yellow bar), mutant alleles of both genes (MUT/MUT; red bar). (B) The plot shows average IOP versus genotypes with or without Tyrpl and Gpnmb mutations in BXD strains at 9.1-13 months $(\mathrm{n}=66)$. 


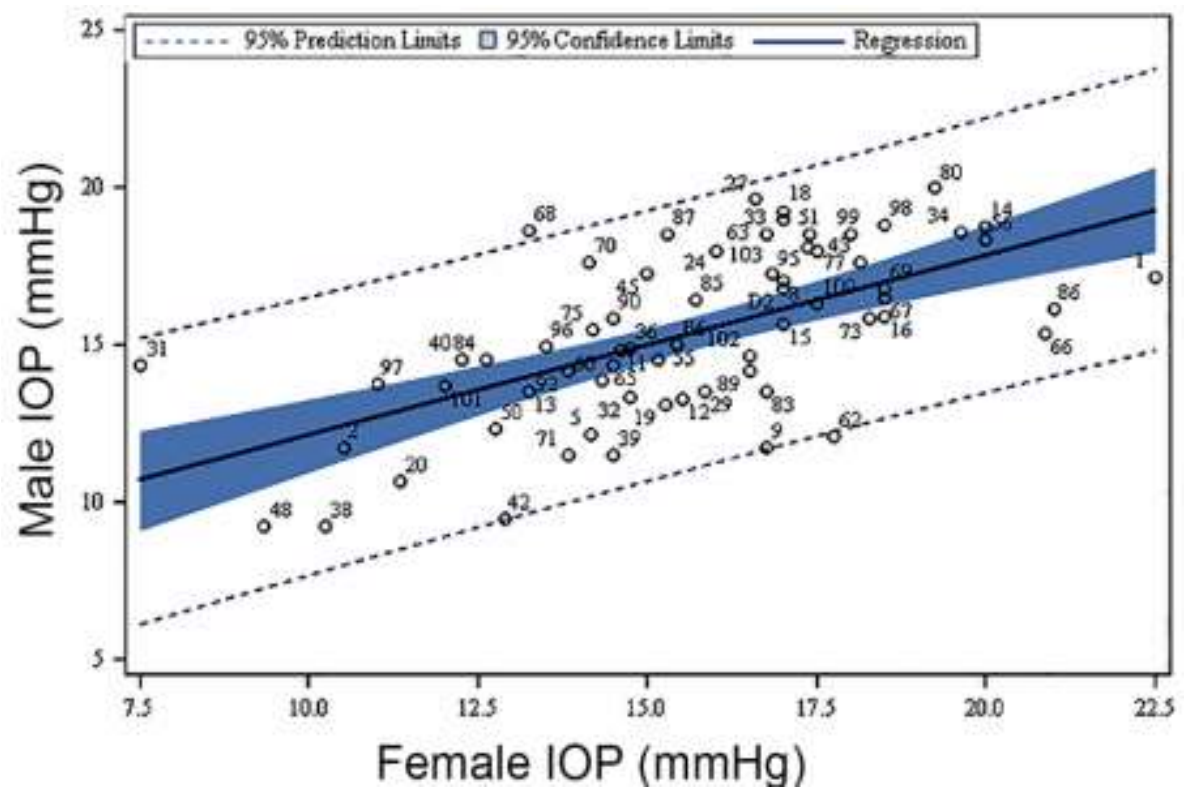

Figure 2-4. Correlation of IOP for females and males at 9.1-13 months

IOP in BXD strains at 9.1-13 months has no statistically significant sex differences and no strong correlation between males and females. 


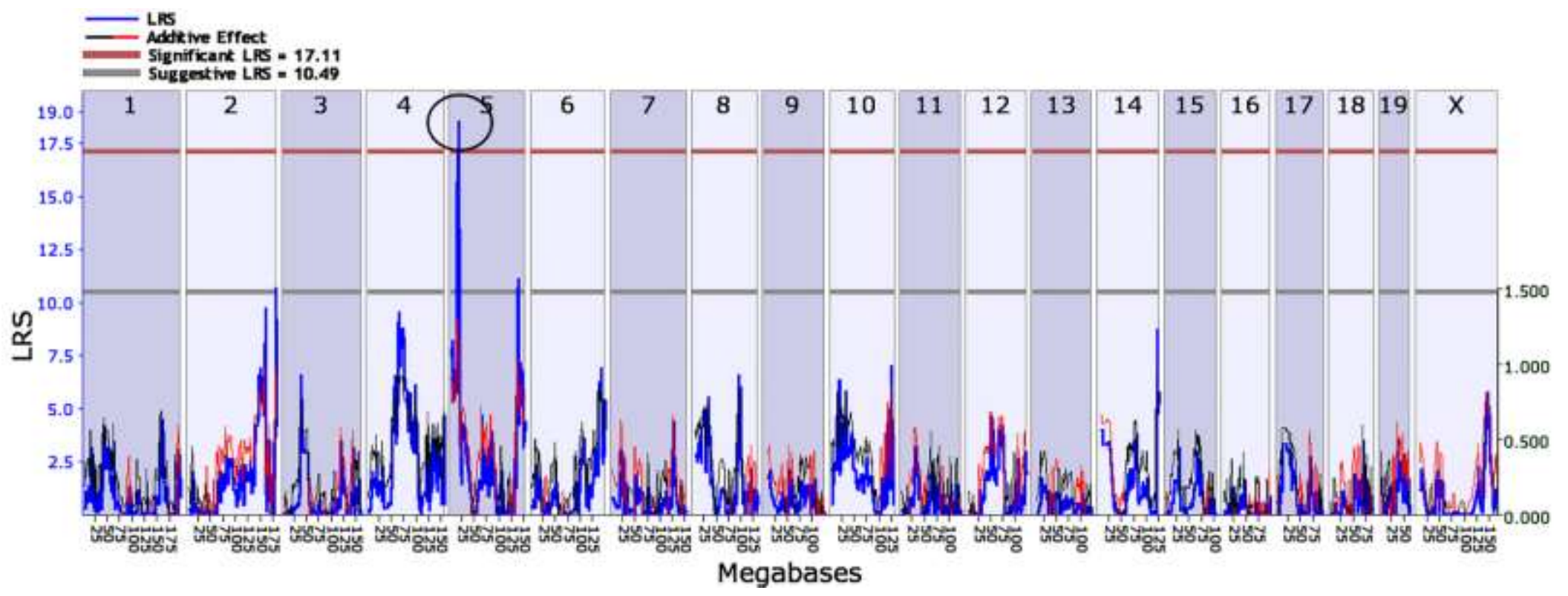

Figure 2-5. QTL mapping for IOP

A significant QTL for IOP is present on chromosome 5. Red and gray horizontal lines dictate significant and suggestive thresholds, respectively. 

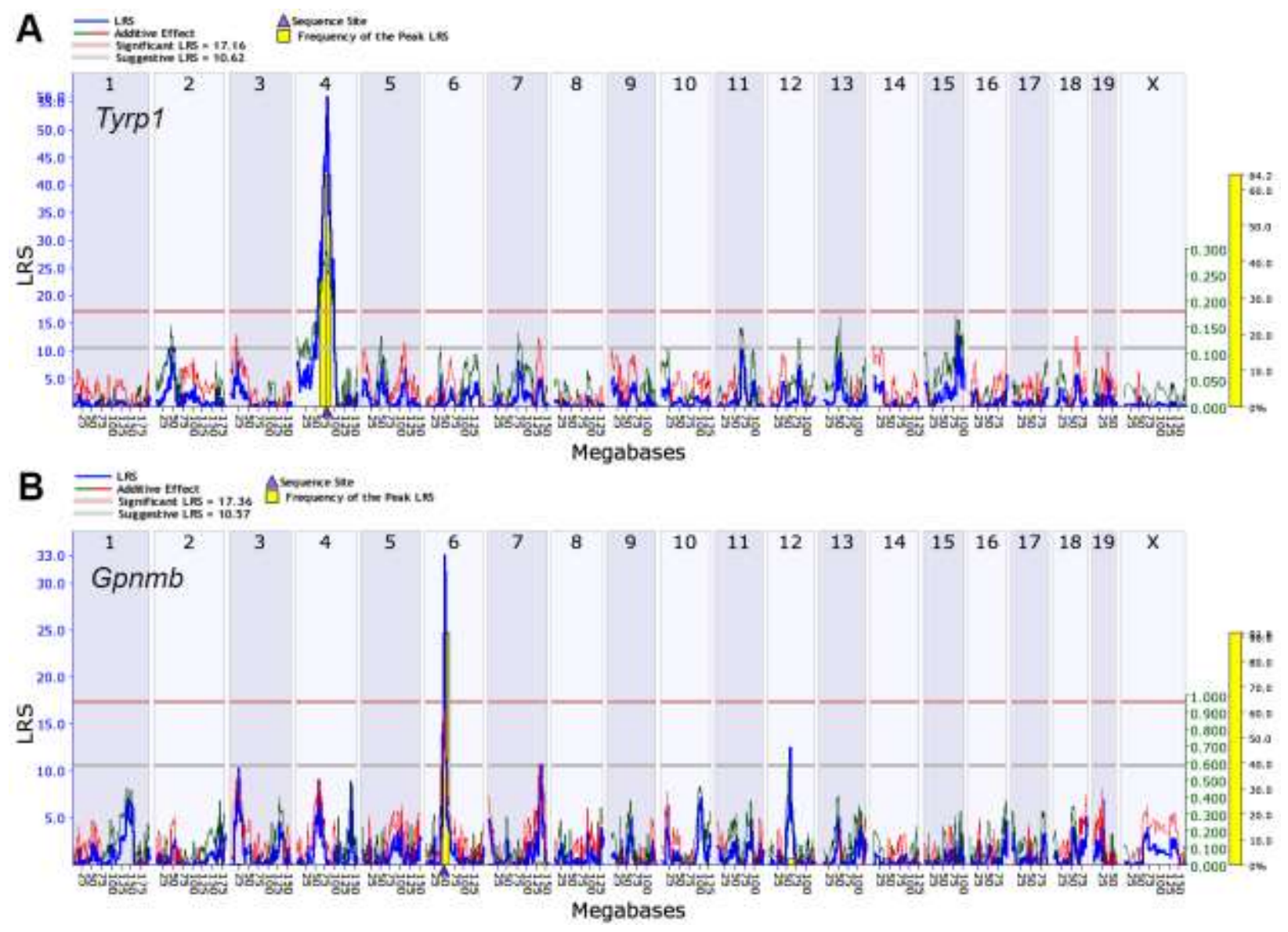

\section{Figure 2-6. QTL mapping for Tyrp1 and Gpnmb in BXD strains eyes}

A significant QTL for Tyrpl is present on chromosome 6. A significant QTL for Gpnmb is present on chromosome 4. Yellow bar represents the frequency of the peak QTL. Red and gray horizontal lines dictate significant and suggestive thresholds, respectively. 
7. plausible biological link to IOP and glaucoma

8. presence of SNPs in the GLAUGEN and NEIGHBOR human GWAS studies of POAG and IOP

To identify the gene candidate, we first performed Pearson correlation by generating a list of top 500 correlates of IOP within the whole eye database based on our published methods (82). Out of top 500 gene correlates, Cacna2d1 (Pearson correlation value: $r=0.440 ; p=0.0003$ ) and Sema3e (Pearson correlation value: $r=0.295 ; \mathrm{p}=0.02$ ) were the only two genes located in the QTL peak at Chr5 (Figure 2-7). To identify the best candidate, we performed partial correlation analysis (120) (Figure 2-8). By controlling for the genetic variability associated with QTL peak at Chr5, we were able to identify a single candidate, $C A C N A 2 D 1$ (partial Pearson correlation value: $\mathrm{r}^{2}=0.331 ; \mathrm{p}=0.0093$ ). CACNA2D1 is known as Calcium Channel, Voltage-Dependent, Alpha 2/Delta Subunit 1 (Figure 2-9). The gene encodes for a preproprotein which is cleaved into multiple chains that comprise the alpha-2 and delta subunits of the voltage-dependent calcium channel complex. Calcium channels mediate the influx of calcium ions into the cell upon membrane polarization (121). Mutations in this gene can cause cardiac deficiencies, including Brugada syndrome and short QT syndrome (122) (Figure 2-10).

\section{Genetic polymorphisms underlying variation in Cacna2d1 contribute to changes in IOP}

The expression of Cacna2d 1 transcript in the whole eye among BXD strains varied (Figure 2-11) with BXD71 having the lowest Cacna2d1 gene expression of $6.91 \pm 0.24$ (expression $\log 2 \pm$ SEM) and BXD48a having the highest at 9.71 \pm 0.33 . The average expression among all BXDs was $8.35 \pm 0.37$. The parental lines, $\mathrm{C} 57 \mathrm{BL} / 6 \mathrm{~J}$ and DBA/2J, had Cacna2d 1 expression levels of $9.5 \pm 0.07$ and $7.54 \pm 0.15$, respectively (Appendix A, Table A-2). The simple interval map shows a significant cis-eQTL for Cacna2dl on proximal Chr5:14.324112 with in the $5 \mathrm{Mb}$ of gene location (Figure 2-12). The maximum LRS score of the locus was 143, which is equivalent to a LOD ratio of 31.01. Cacna2d1 gene has a $69 \%$ variance, which is explained by the single peak QTL at Chr5. The majority of BXD strains with the D parental allele have low expression of Cacna2dl in the eye, while those with the B parental allele have higher expression of the gene (Figure 2-13). Cacna2dl is highly polymorphic in both mice and humans. We identified known genetic variability between C57BL/6J and DBA/2J strains in Cacna2dl gene and its flanking region within the eye using UCSC genome bioinformatics site (http://genome.ucse.edu) (Figure 2-14). We identified five synonymous coding SNPs within Cacna2d1 gene, 866 non-coding SNPs and 177 insertions/deletions, making this gene highly polymorphic (Table 2-1). These polymorphisms segregate among the BXD strains. Similarly, in humans, $C A C N A 2 D 1$ has 8801 exonic SNPs, 44 of which are nonsynonymous. There are also $7 \mathrm{CNVs}$ and 5 InDels segregating in humans [http://www.genecards.org/cgibin/carddisp.pl?gene=CACNA2D1] (Table 2-2).

The next step in our analysis was to obtain SNP level p-values for association with GLAUGEN and NEIGHBOR human GWAS studies of POAG and IOP (116). 


\begin{tabular}{|c|c|c|c|c|c|c|c|c|c|c|c|c|c|c|}
\hline Inder & $\begin{array}{l}\text { Record } \\
10\end{array}$ & $\begin{array}{l}\text { Symbot } \\
\text { ase }\end{array}$ & $\begin{array}{l}\text { Desecription } \\
\text { ase }\end{array}$ & $\begin{array}{l}\text { Location } \\
\text { Cor and }\end{array}$ & Mean & Max & $\begin{array}{l}\text { Max LRs Location } \\
\text { Car and Mb? }\end{array}$ & Sample & Cosses & $\begin{array}{l}\text { Sample } \\
\mathrm{P}(\mathrm{s})\end{array}$ & Carr: & Tiksou & $\begin{array}{l}\text { Thissue } \\
\mathrm{P}(9)\end{array}$ & $\begin{array}{l}\text { eng } \\
\text { Cunk } \\
\end{array}$ \\
\hline 3230 & 1416036 _at & thet & 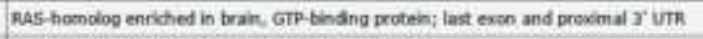 & Cors: 24.J09116 & 12.573 & 130 & Ohr21 178.942786 & -0.312 & 61 & 0.01406 & - & $\pi$ & $=$ & 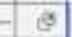 \\
\hline 324 覀 & 1422045 andent & reant2 & wrotesh triocine phosphatase, non-ecester type 12 & ON5: 20.492590 & 10.399 & 10.4 & Oris: 16.221091 & -9.312 & 61 & 0.01396 & - & - & - & s) \\
\hline 325 & 1455105_at & Penon 12 & 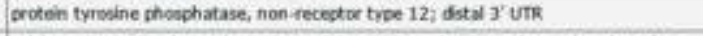 & Cor5: 20.492484 & 10.607 & 14.8 & Chrs: 18.648049 & -0.320 & 61 & 0.01162 & - & - & - & -3 \\
\hline 3260 & 1440397_at & Cacnazöt & 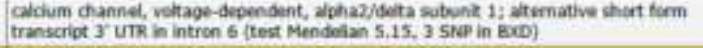 & Curs: 15.773869 & 5,514 & 73.2 & Cors: 14.324112 & 0.305 & 61 & 0.01632 & - & - & 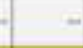 & is \\
\hline 3278 & 1446324_at & cantezat & 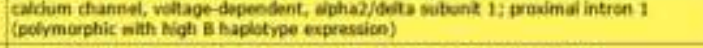 & Chrs: 1: & B.533 & 143.0 & Ors: 14.324112 & 0.440 & 61 & 0.00033 & - & - & - & s \\
\hline $325 \%$ & 1442225 _at & Sema3e & 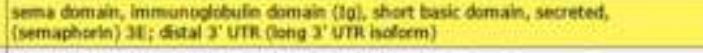 & Cors: 14.256145 & 8.534 & 28.4 & Cor5: 12.998375 & 0,205 & 61 & 0.02065 & 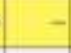 & - & 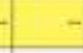 & 党 \\
\hline 3290 & 141971 Jat & semase & 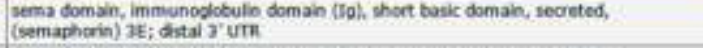 & ars: 14.253727 & 8.634 & 26.5 & .042123 & 0.309 & bi & 0.01496 & - & - & - & \$ \\
\hline $330 \mathrm{a}$ & 1427673 _a_at & Semaje & 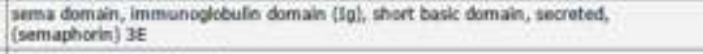 & Oros: 14.253239 & 7.942 & 1500 & Ohrs: 14.224112 & 0.347 & 61 & 0.00587 & - & - & - & s) \\
\hline 10 & 1431000_at & I500011466aik & RDEEN CONA ISOD0: 1K16 Dent & Ore4: 155.091497 & 7,671 & 85.5 & Oret: 155.235046 & 0.204 & 61 & 0.02102 & - & - & - & 8 \\
\hline
\end{tabular}

Figure 2-7. The screen shot depicts the Pearson correlation analysis using the tools available on GeneNetwork

Pearson correlation analysis listed two genes- Cacna2dl and Sema3e within the QTL peak. The genes highlighted in yellow depicts top candidate genes for IOP modulation at 9.1-13 months of age based on Pearson correlation analysis. 
Primary Trait

Unpublished : RecordiD/16340 - Eye, visual system, physiologyt Intraocular pressure (IOP), 9.1 to 13 months old, both sexes, average of left and right eyes, Merged. by Hong $L$, and colleagues -- FROM : Bxin Puthishent Phenotypes Database

Control Traits

1. Locus rs13478120 [Chr 5 e 17.827 Mb] -... FRoM : : Bxo Genotypes Databast

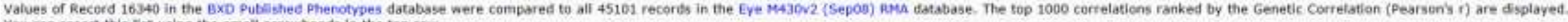
You can rasort this list using the small arrowheads in the top row.

Click the correlation values to generate scatter ploss. Select the Record id to open the Trait Data and Analysis form. Select the symbol to open NCBI Entrex.

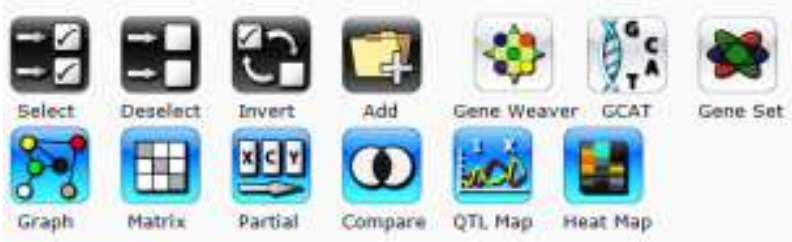

\section{Mare Ontand \\ Domeload tabilit}

\begin{tabular}{|c|c|c|c|c|c|c|c|c|c|c|c|c|c|c|c|}
\hline & $\begin{array}{l}\text { Record } \\
\text { ase }\end{array}$ & $\begin{array}{l}\text { syninbel } \\
\text { cas }\end{array}$ & $\begin{array}{l}\text { Descrivition } \\
\text { ain }\end{array}$ & $\begin{array}{c}\text { arr } \\
\omega=\end{array}$ & mo & Mean & $\begin{array}{l}\text { N. } 5 \\
0 ?\end{array}$ & $\begin{array}{l}\text { Salmple } \\
\text { Partible }\end{array}$ & $\begin{array}{l}\text { Somple } \\
\text { p(partialn) }\end{array}$ & $\begin{array}{l}\text { Sample } \\
\text { if }\end{array}$ & $\begin{array}{l}\text { Sample } \\
\text { p(g) }\end{array}$ & $\begin{array}{l}\text { detith } \\
\text { ise }\end{array}$ & $\begin{array}{l}\text { Putbined } \\
\text { in }\end{array}$ & $\begin{array}{l}\text { Thsoe } \\
\text { E }\end{array}$ & $\begin{array}{l}\text { Tiksoe } \\
\mathrm{p}(\mathrm{g})\end{array}$ \\
\hline 670 & 1430691_at & 4632411 POBRK & RDY.EN CONA 4632411008 gane & s & 29.716432 & 5.569 & 58 & -0.272 & 0.03634 & 0.312 & 0.01725 & 0.040 & N/A & N/A & $\mathrm{N} / \mathrm{A}$ \\
\hline $680 \square$ & 1425605_a_at & Lmer2 & limb redion 1; 2x exons near 3' and of message & s: & 29.579305 & 0.034 & 58 & 0.345 & 0.00641 & 0.365 & 5.00048 & -0.020 & N/A & N/A & N/A \\
\hline 0810 & 1410036_at & Aheb & 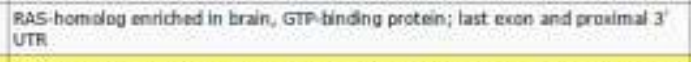 & 5 & 24,309116 & 12.575 & 56 & -0.310 & 0.01572 & 0.349 & 90.00718 & 0.040 & $N / A$ & $\mathrm{~N} / \mathrm{A}$ & N/A \\
\hline 6822 & 1425861_x_at & Cacnazdi & caldum channel, volkage dependent, alphaz; detta subunit 1 ; dstal or far $3^{\prime}$ UTR & s & 15.876934 & 6.231 & $5 a$ & 0.331 & 0.00926 & 0.299 & 0.02239 & 0.032 & N/A & N/A & $N / \mathrm{A}$ \\
\hline 683 & 144598__at & coselt & oydin-dependent knase 14 & 5. & 4.979172 & 7.245 & se & 0.298 & 0.02051 & 0.213 & 0.10126 & -0.005 & N/A & N/A & $N / A$ \\
\hline $684 a$ & 1422534_at & CoDStat & 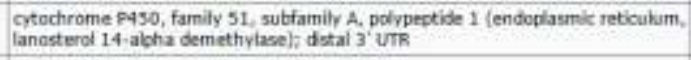 & 5 & 4.090269 & 6.336 & 56 & -0.368 & 0.00337 & -0.155 & 50.24639 & -0.213 & N/A & N/A & $\mathrm{N} / \mathrm{A}$ \\
\hline oes 0 & 1459926_at & Fon 1336 & Family with Sequence similarty 133 , mamber 8 & 5 & 3.505236 & 6.744 & 50 & -0.305 & 0.01775 & -0.289 & 0.02775 & -0.015 & $N / \mathrm{A}$ & N/A & N/A \\
\hline 680 & 143394s_at & Flazgrb & phosshaliesse A2, arowip 18, pancreas & 5 & 0.0 & 5,302 & 56 & 0.281 & 0.02593 & 0.270 & 0.04040 & 0.011 & N/A & $N / A$ & N/A \\
\hline
\end{tabular}

Figure 2-8. The screen shot depicts the partial correlation analysis using the tools available on GeneNetwork

Partial correlation analysis listed only Cacna2d1 within the QTL peak. The genes highlighted in yellow depicts top candidate gene for IOP modulation at 9.1-13 months of age based on partial correlation analysis. 


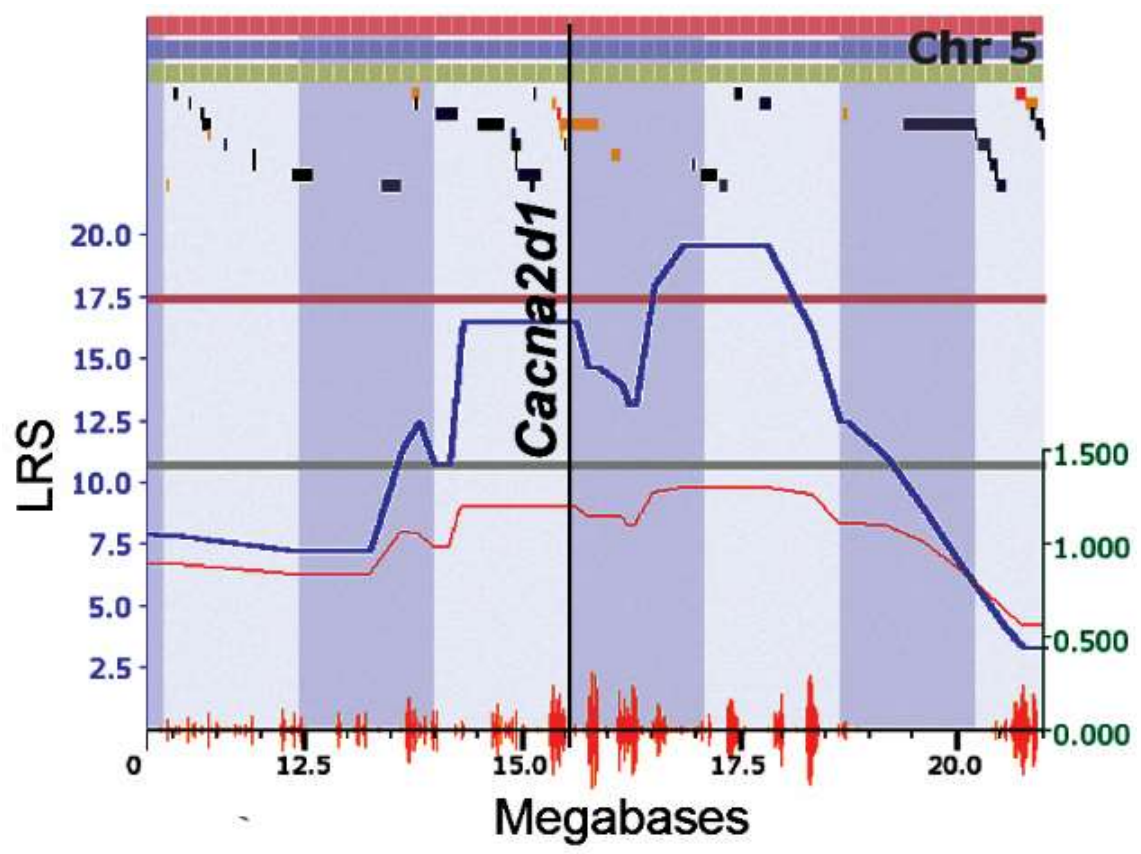

Figure 2-9. Zoomed-in view of QTL map for IOP

A significant QTL for IOP is present on chromosome 5 between $14-19 \mathrm{Mb}$. Cacna2dl is the candidate gene located in the peak QTL. Black vertical line shows the location of the candidate gene. Red and gray horizontal lines dictate significant and suggestive thresholds, respectively. 


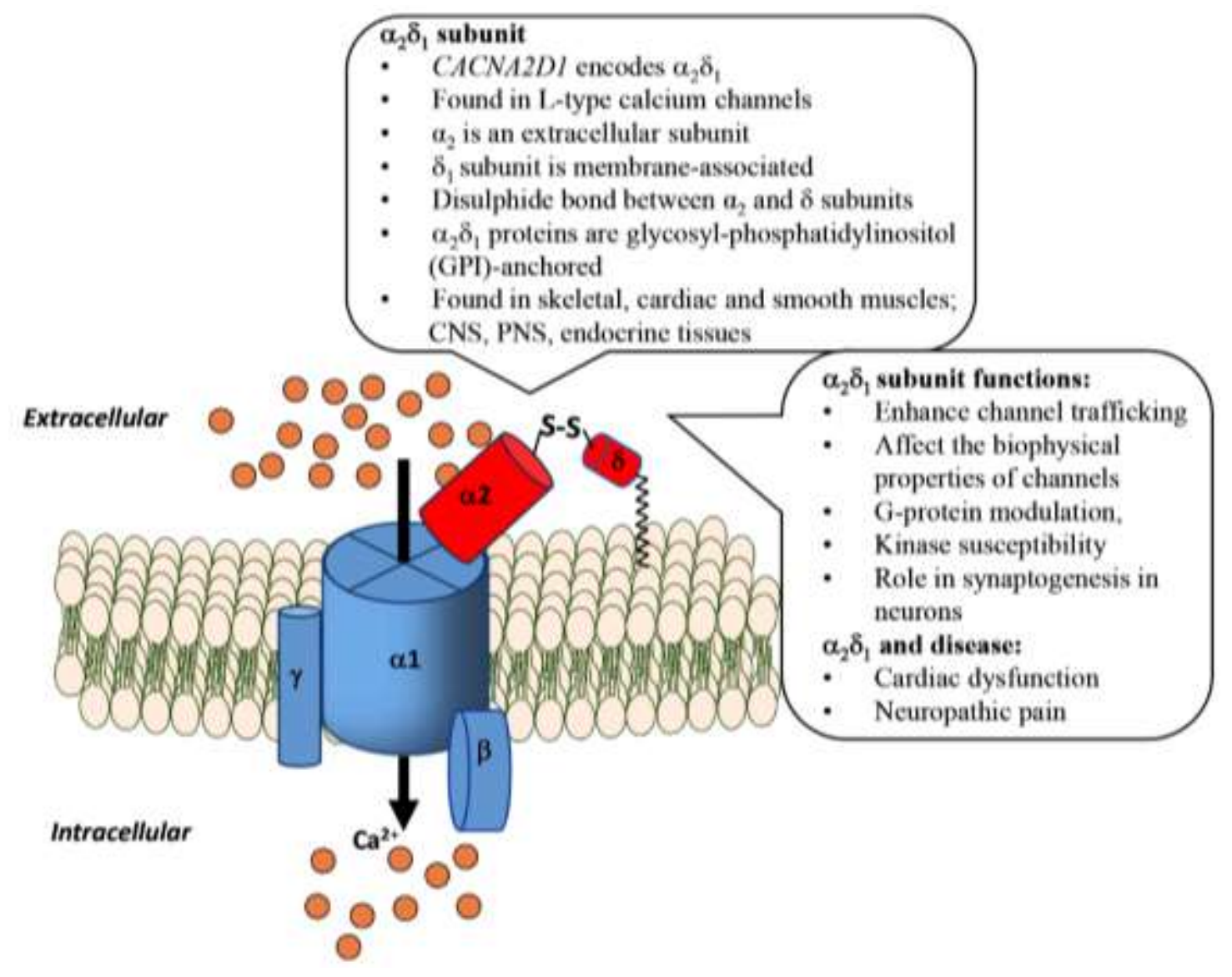

Figure 2-10. Subunit structure of $L$ type calcium channel with description.

$\alpha_{1}, \beta$ and $\gamma$ subunits are in blue. $\alpha_{2}$ and $\delta$ subunits are in red with a disulphide bond between them. $\alpha_{2} \delta_{1}$ is GPI anchored to the plasma membrane. 


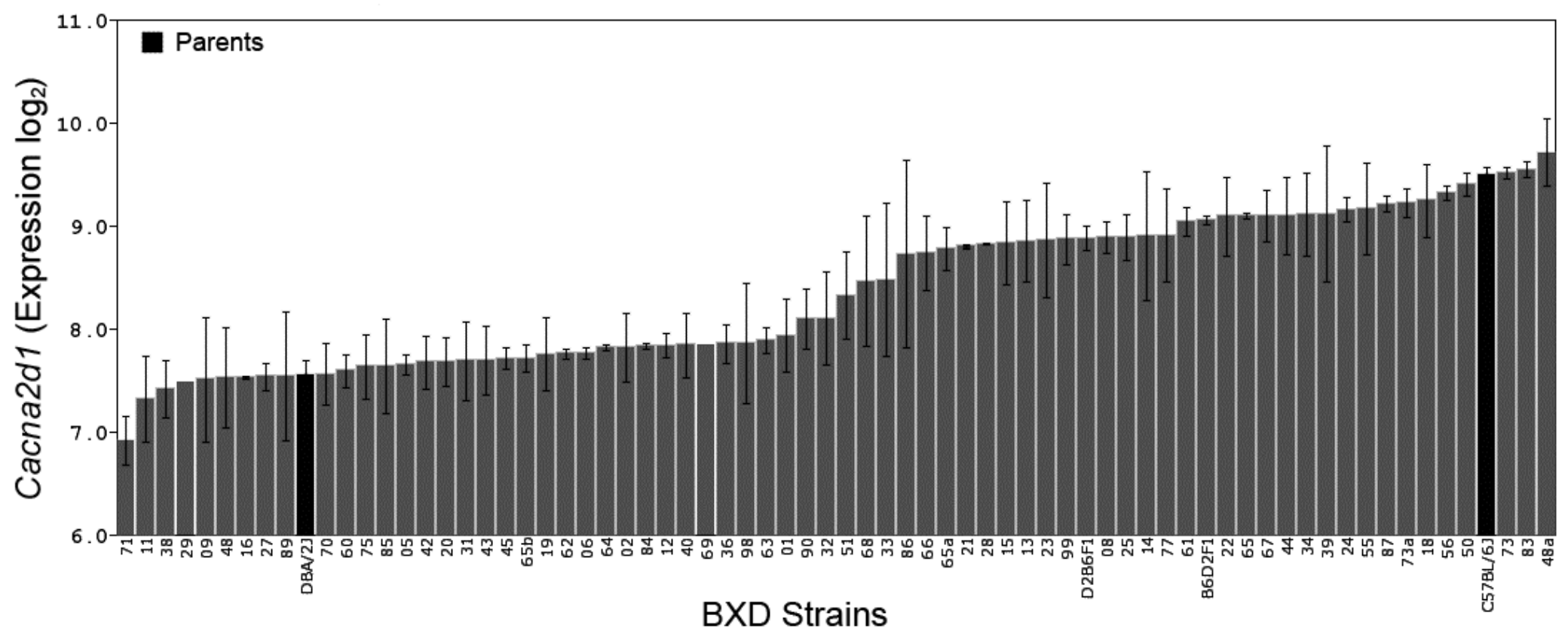

Figure 2-11. mRNA rxpression levels of Cacna2d1 across BXD strains and parental strains.

Rank-ordered mean Cacna2d1 mRNA expression levels across the BXD recombinant inbred family. Values denote normalized relative expression levels on $\log _{2}$ scale (mean \pm SEM). 

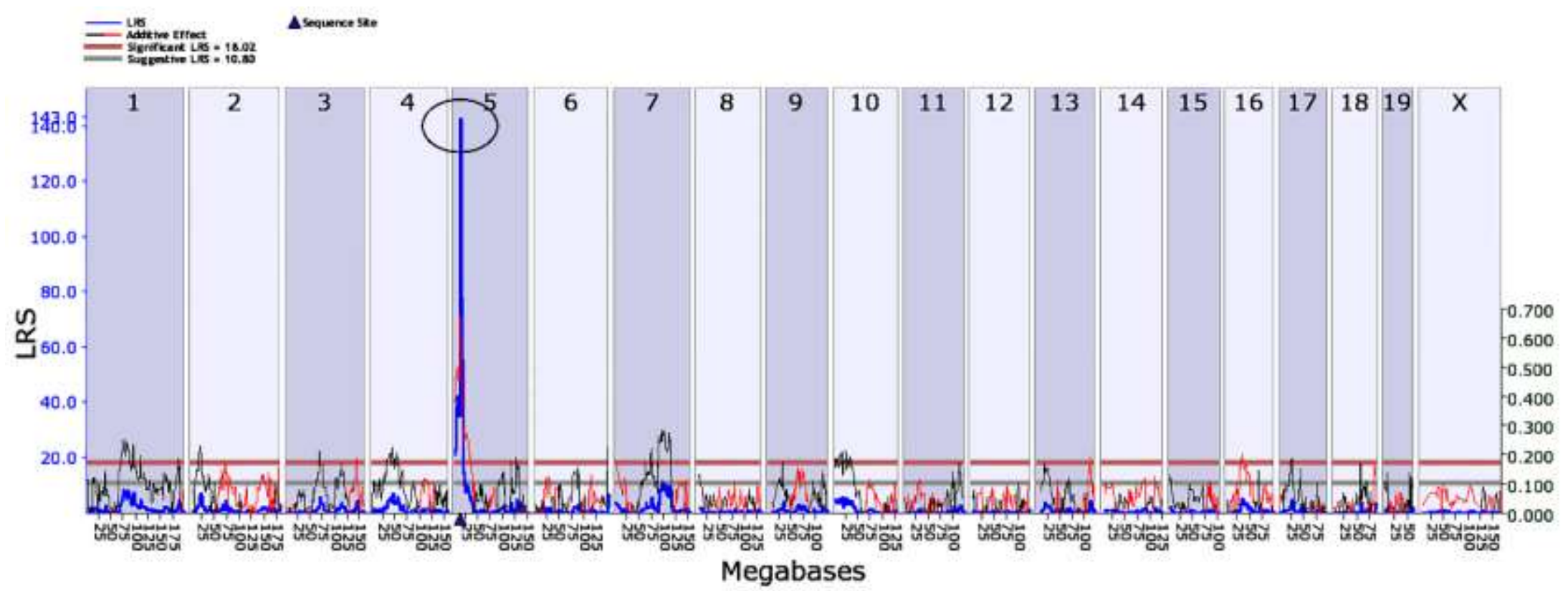

Figure 2-12. A significant cis-eQTL for Cacna2d1 is present on chromosome 5 at 14-19 Mb.

Red and gray horizontal lines dictate significant and suggestive thresholds, respectively. The purple triangle indicates the location of Cacna2dl within the mouse genome. 


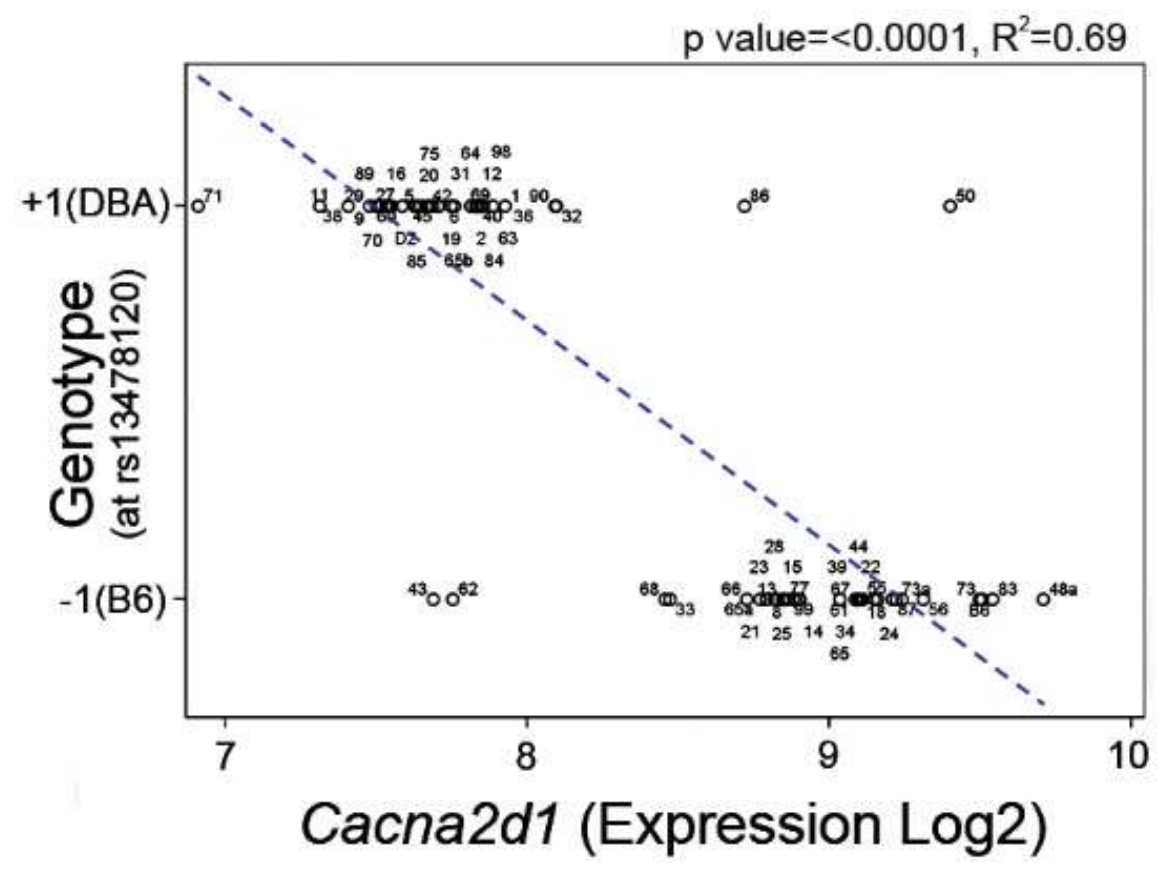

Figure 2-13. Cacna2d1 expression in whole eye of BXD strains attributable to the genotype

Correlation between the trait values (here, Cacna2dl expression levels) and the genotype at the peak location. The majority of BXD strains with the D parental allele have low expression of Cacna2d1 in the eye, while those with the B parental allele have higher expression of the gene. 


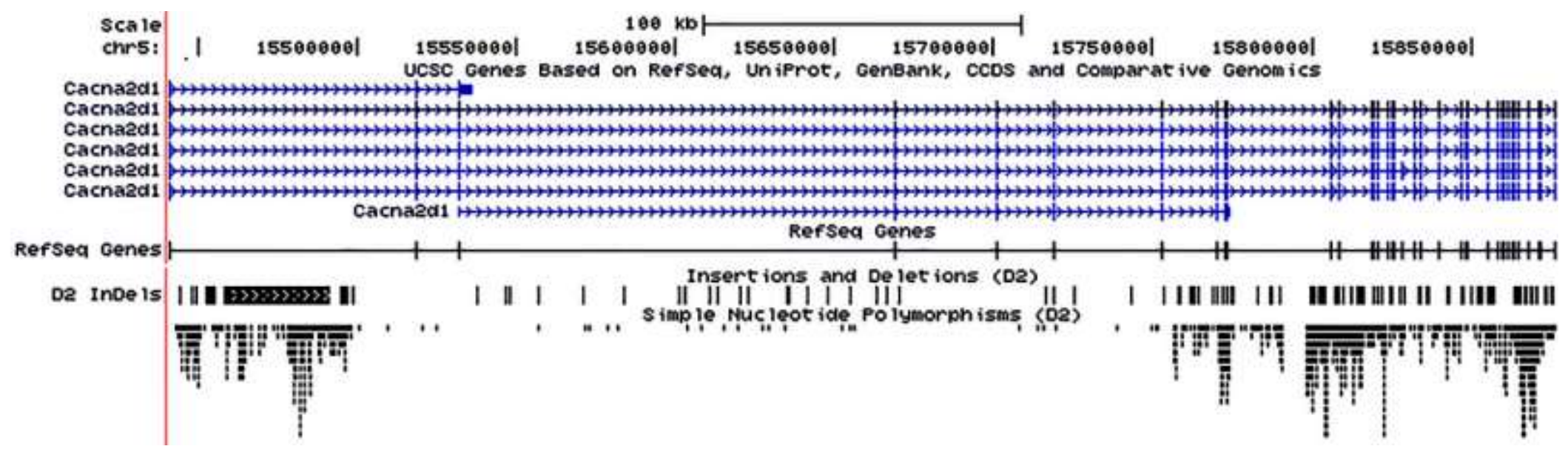

Figure 2-14. UCSC Genome Browser illustration of the gene structure and location of SNPs associated with Cacna2d1 gene

Graphic depiction of Cacna2d1 gene with SNPs on chromosome 5 of the mouse genome. Reference Sequence mRNAs are represented in blue and known mRNAs are represented in black. 
Table 2-1. Summary of candidate gene Cacna2d1 in mouse

\begin{tabular}{|c|c|c|c|c|c|c|c|}
\hline $\begin{array}{c}\text { Candidate } \\
\text { Gene }\end{array}$ & Probe Set I.D & $\begin{array}{c}\text { Gene } \\
\text { Location }\end{array}$ & $\begin{array}{c}\text { Mean } \\
\text { Expression } \\
\text { Level }\end{array}$ & $\begin{array}{l}\text { Max LRS and } \\
\text { Location }\end{array}$ & $\begin{array}{c}\text { Cis eQTL } \\
\text { (Yes or No) }\end{array}$ & $\begin{array}{l}\text { SNPs and } \\
\text { Indels }\end{array}$ & P-Value \\
\hline Cacna2d1 & 1446324_at & $\begin{array}{c}\text { Chr5: } 15,440,509- \\
15,880,329 \text { bp }\end{array}$ & 8.5 & 143 & Yes & $\begin{array}{l}\text { - Non-coding } \\
\text { SNPs: } 866 \\
\text { - Synonymous } \\
\text { SNPs: } 5 \\
\text { - InDels: } 177 \\
\end{array}$ & $2.43 \times 10^{-5}$ \\
\hline
\end{tabular}

Table 2-2. Summary of candidate gene Cacna2d1 in humans

\begin{tabular}{|c|c|c|c|c|}
\hline $\begin{array}{c}\text { Candidate } \\
\text { Gene }\end{array}$ & Location & SNPs and Indels & $\begin{array}{l}\text { SNP } \\
\text { I.D }\end{array}$ & P-Value \\
\hline$C A C N A 2 D 1$ & $\begin{array}{l}\text { Chr 7: } \\
81,946,444- \\
82,443,798 \\
\text { bp }\end{array}$ & $\begin{array}{l}\text { - Coding SNPs: } 398 \\
\text { - Non-coding SNPs: } \\
\text { 17,940 } \\
\text { - InDels: } 45\end{array}$ & rs4732474 & 0.0075 \\
\hline
\end{tabular}


Association analyses were carried out using the SNP genotypes of the CACNA2D1 gene and flanking regions. The results showed evidence of weak associations between rs $1022418(\mathrm{p}=0.03$; Odds ratio=1.17) of the $C A C N A 2 D 1$ and normal tension glaucoma subset as well as rs6467882 $(\mathrm{p}=0.04$; Odds ratio $=0.87)$ of the $C A C N A 2 D 1$ and high tension glaucoma subset. Furthermore, based on the inputted genotypes, rs4732474 of $C A C N A 2 D 1$ showed a genome wide nominal association with the combined normal tension and high tension glaucoma populations (i.e POAG). The p-value of rs4732474 was 0.0075 and odds ratio was 1.14 , which is significant at gene level. None of the SNPs remained genome wide significant after correction for multiple testing even when linkage disequilibrium structure of the SNPs was taken into account, however rs4732474 was significant at gene level since the p-value was less than 0.05 .

\section{CACNA2D1 is localized in the mouse and human eye}

To compile additional evidence to support our hypothesis, we performed immunohistochemistry (IHC) to determine the localization pattern of CACNA2D1 in mouse and healthy human donor eyes. In mouse retina, CACNA2D1 is prominently localized in the trabecular meshwork, ciliary body and ciliary muscle (Figure 2-15). CACNA2D1 immunoreactivity was also observed in the nerve fiber layer, inner plexiform layer and outer nuclear layer was also occasionally observed (Figure 2-15). Positive staining and localization of CACNA2D1 in the mouse eye was further corroborated by immunofluorescence microscopy analysis in human donor eyes (Figure 2-16).

\section{Discussion}

POAG is often regarded as 'thief of sight' and IOP is a highly heritable risk factor POAG, this makes is the only target for current glaucoma therapy (14). But the genetic factors which determine IOP are largely unknown. In this investigation, we have combined cutting edge methodologies of systems genetics, bidirectional studies using multiple species, meta-analyses and immunohistochemistry studies to identify a novel genetic modulator of IOP. We systematically explored sources of genetic variation in the IOP. We identified a novel QTL for IOP on chromosome 5. By combining our QTL analyses with expression correlation analyses, human GWAS and literature review, we identified a cis-regulated gene-Cacna2dl that may enable a deeper understanding of IOP and glaucoma.

POAG has an insidious onset and low natural course hindering a definite diagnosis at an early stage. Diagnosis is usually non-uniform due to etiological and clinical heterogeneity of POAG. Due to this, the current gene-finding in POAG focuses on the adult-onset POAGs where relatively few people would be available to participate thus limiting the identification of large affected families for gene mapping by linkage analysis (123). These limitations fuel the need for the use of a genetic reference population of RI strains, from which diverse phenotypes and genotypes can be collected 

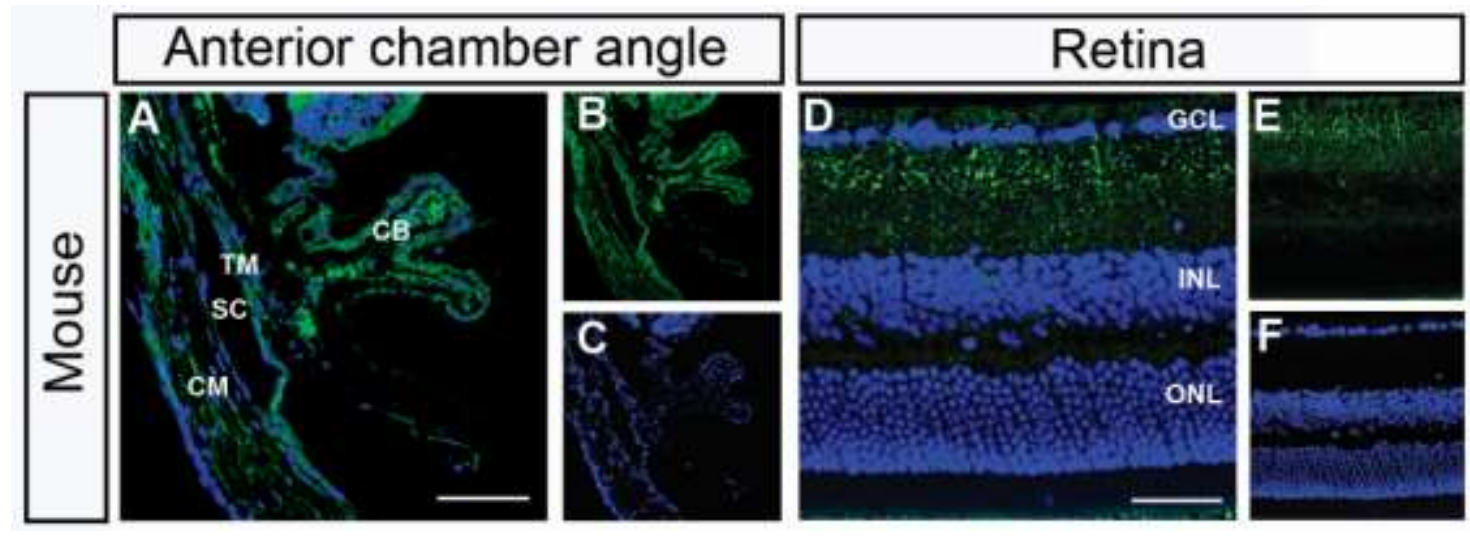

Figure 2-15. Cellular localization of CACNA2D1 in C57BL/6J mouse eye

Sections from C57BL/6J mouse anterior chamber angle and retina were labeled with antibodies against CACNA2D1. TO-PRO-III iodide staining labeled nuclei of all retinal cells (blue). Abbreviations: $\mathrm{TM}=$ trabecular meshwork; $\mathrm{CB}=$ ciliary body; $\mathrm{CM}=$ ciliary muscle; $\mathrm{SC}=$ schlemm's canal; $\mathrm{GCL}=$ ganglion cell layer; $\mathrm{INL}=$ inner nuclear layer; $\mathrm{ONL}=$ outer nuclear layer. Scale bar: $100 \mu \mathrm{m}$.
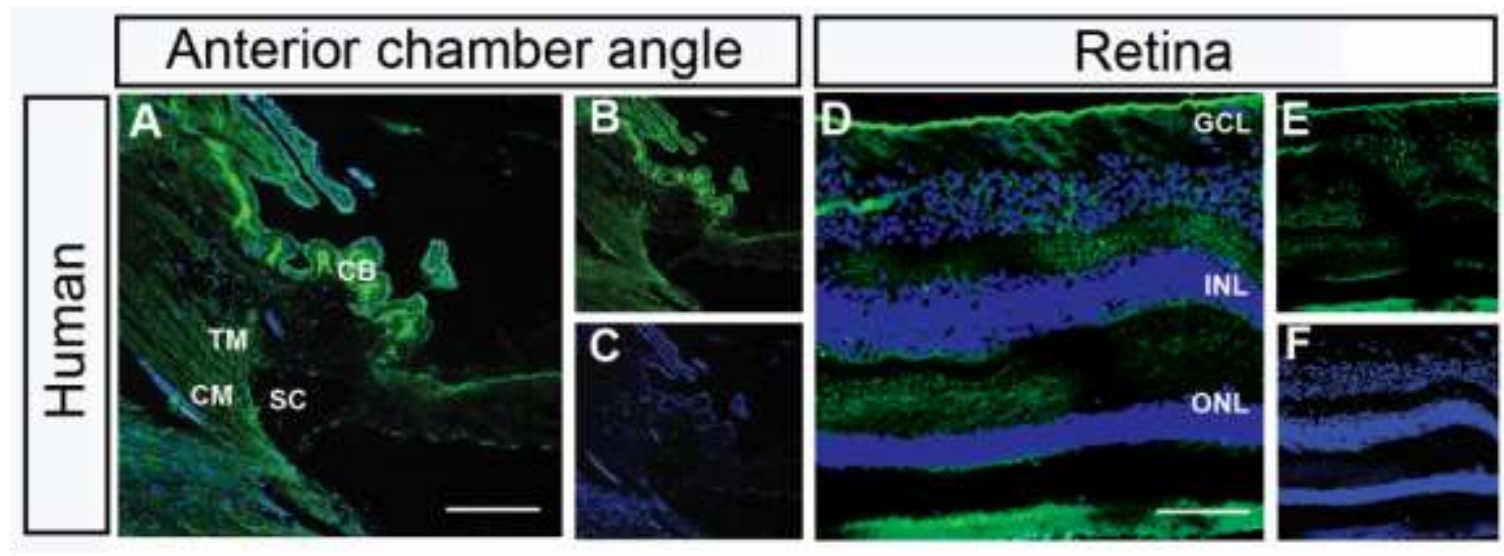

Figure 2-16. Cellular localization of CACNA2D1 in a human donor eye with no diagnosis of ocular diseases

Sections from the anterior chamber angle and retina were labeled with antibodies against CACNA2D1. TO-PRO-III iodide staining labeled nuclei of all retinal cells (blue).

Abbreviations: $\mathrm{TM}=$ trabecular meshwork; $\mathrm{CB}=$ ciliary body; $\mathrm{CM}=$ ciliary muscle; $\mathrm{SC}=$ schlemm's canal; $\mathrm{GCL}=$ ganglion cell layer; $\mathrm{INL}=$ inner nuclear layer; $\mathrm{ONL}=$ outer nuclear layer. Scale bar: $20 \mu \mathrm{m}$. 
and reproduced over time. Using the BXD RI genetic reference population, we found that IOP is highly heritable and variable, there was no gender influence in IOP at 9.1-13 months.

To determine which candidate genes modulate IOP in the eye, we performed simple interval mapping. Our mapping studies in BXD mice indicate a single highly genome wide significant QTL on Chr5. Variation in IOP in the BXD family maps to Chr 5 with a LOD of 4.0 and a 1.5 LOD confidence interval from 14.2 to $18.8 \mathrm{Mb}$ (Pclo to A921504A21Rik). The effect size is $+1.0 \mathrm{mmHg} /$ per B allele. We pursued second strategy of Pearson and partial correlation analyses to corroborate the findings of our QTL analysis for Chr5. These results suggested that within this strong eQTL reside some transcripts that predominantly regulate IOP. We focused on one positional and biologically relevant gene-Cacna2dl-located on Chr5 QTL peak. Cacna2dl is the best candidate and is cis-regulated in BXD retinal data sets. This gene has shown to play key roles in modulating calcium uptake which is crucial for IOP in the eye (124). Tyrp1 and Gpnmb gene mutations have been well known to develop iris transillumination defect in $\mathrm{DBA} / 2 \mathrm{~J}$ mice. However, based on our correlation analysis and previously published paper there was no correlation between the expression of these genes and IOP. Moreover, IOP did not map to either the Tyrpl or Gpnmb locus (119).

Genetic variations are a major factor for inter-individual differences in disease susceptibility and response to environmental exposures. The great majority of DNA polymorphisms consist in SNPs. Most SNPs manifest in introns which may have no effect on the individual phenotypes. However, SNPs, though rarely being directly pathogenic, are frequently correlated to the occurrence of multigenic diseases. Both in mouse and humans Cacna2dl has hundreds of SNPs and insertions/deletions, which makes it highly polymorphic. In humans, CACNA2D1 also contained a SNP with a $\mathrm{p}$ value reaching significance at the individual gene level in the GLAUGEN and NEIGHBOR human GWAS studies of POAG and IOP (SNP rs4732474, $\mathrm{p}=0.0075$ ). Recently, a GWAS of Beaver Dam Eye Study revealed 7 regions of interest modulating IOP. One of the regions of interest is Chr7 which corresponds to CACNA2D1 gene and this region has suggestive association with IOP (11). Another study has shown human Chr 7 (i.e., 7q35-q36) to be associated with pigment dispersion syndrome (125). A common variant mapping to another calcium channel subunit-CACNA1A, is associated with susceptibility to exfoliation syndrome (126). In this disease, there is a build-up of extracellular matrix deposits and pigment in the trabecular meshwork which can impede the drainage of $\mathrm{AH}$ from the eye thus resulting in elevated IOP and glaucomatous optic neuropathy (126). This provides further evidence support to our hypothesis that $C A C N A 2 D 1$ may modulate structure or function of calcium channels and contribute to modulation of IOP in glaucoma. Our immunohistochemistry data demonstrated that $C A C N A 2 D 1$ is localized in the ciliary body, trabecular meshwork and ciliary muscle as well as in the RGC bodies and axons. This observation was consistent in both mouse and human eyes. An interesting implication of this observation is that CACNA2D1 might be modulating IOP via the AH dynamics and is neuroprotective as well, although the mechanism by which this occurs is not known. 
CACNA2D1 encodes for the alpha2 delta1 subunit of the type L-type voltagedependent calcium channel. Calcium channels are responsible for the transport of calcium ions across cell membranes and play a key role in a cell's ability to generate and transmit electrical signals. Calcium channels are ubiquitous and are responsible for the transport of calcium ions across cell membranes which plays a key role in a cell's ability to generate and transmit electrical signals. They play important physiological roles in modulating the calcium balance in various tissues (121). In the eye, calcium plays a critical role in modulating the cleavage of calcineurin in response to elevated IOP, which inhibits pressure-induced RGC axon loss (127). In addition, calpains activate cyclin dependent kinase 5, which has also been implicated in POAG (128). Mishandling of intracellular calcium by glaucomatous trabecular meshwork cells may also contribute to its failure, leading to increased AH outflow resistance and elevated IOP (124). Excessive calcium influx is a major player of retinal cell death and a hallmark of pathological processes activated by retinal injury and disease (129). RGC loss due to dysregulated $\mathrm{Ca}$ channel signaling has been attributed to glutamate excitoxicity mediated by overstimulation of NMDA receptors, (130), activation of $\mathrm{Ca}^{2+}$ permeable TRP channels (131), defective calcium buffering stores (for example, mitochondrial dysfunction) (132), and excessive release of nitric oxide (133). Since functional deficits can occur early in the progression of glaucoma and other optic neuropathies, targeted Ca channel blockade may have a role to play in how optic neurpathies are treated. Understanding the role of CACNA2D1 in RGCs will provide a platform for future experiments investigating their contribution in retinal injury models. Because calcium channels play a role in the pathophysiology of glaucoma, systemic calcium channel blockers (CCBs) have been evaluated in a limited number of studies as plausible therapies for POAG (134). However, the results have been inconsistent, with some studies demonstrating that CCBs are highly effective in lowering IOP, protecting ganglion cells, increasing ocular blood flow and improving visual function, while others fail to replicate those results (134). It is possible that because CCBs target calcium channels, which are highly polymorphic, they will only be effective on a subset of the population.

Our study provides the first candidate gene- $C A C N A 2 D 1$, to explain variation in IOP by combining the power of mouse quantitative genetics with human GWAS studies. This novel finding will lead to the development of new IOP-lowering therapy. In future, similar studies would enable us to identify glaucoma-associated genes and how these genes can be validated as functional targets, and how this will bridge the gap between data generation and effective drug target identification. 


\section{CHAPTER 3. MULTIPRONGED APPROACH TO IDENTIFY AND VALIDATE A NOVEL UPSTREAM REGULATOR OF SNCG IN MOUSE RGC*}

\section{Introduction}

Glaucoma is the world's leading retinal neurodegenerative disease that causes irreversible vision loss (135). Degeneration of RGC somas and their axons is a hallmark of its pathophysiology. Because it is a multifactorial disease, the degeneration of the RGC can be influenced by multiple affecters including physiological stress (i.e. oxidative stress, neurotransmitter toxicity (136), and/or ER stress-related chaperone proteins (137, 138). Members of the synuclein family of proteins are chaperone molecules predominantly expressed in neurons (139). In diseases such as Alzheimer's and Parkinson's, mutant synucleins are key components of pathological inclusions $(140,141)$. Among the three known isoforms $(\alpha, \beta$ and $\gamma)$, a reduction in the expression level of $\gamma$ synuclein (SNCG) in the retina and optic nerve head have been reported in glaucoma patients $(139,142)$. Recent studies have demonstrated a direct correlation between the reduced expression of Sncg mRNA and the loss of RGCs in various animal models of glaucoma (143). Collectively, these studies suggest that loss of SNCG is a putative marker of RGC degeneration, yet the molecular targets and biological relevance of aberrant SNCG expression remains largely unknown.

In the present study, we used $\mathrm{C} 57 \mathrm{BL} / 6 \mathrm{~J}$ and DBA/2J mice, as well as the BXD RI family as a collective genetic reference panel to determine which gene(s) regulate $S n c g$ expression in murine RGCs. We have also compared the localization pattern of Sncg and its upstream modulator in mouse and human donor retinae using immunohistochemistry. Using flow cytometry-based sorting, we isolated enriched RGCs and performed knockdown studies to validate our findings. The outcomes of our investigation may provide clues to understanding the molecular mechanisms that account for the degenerative changes in RGCs in glaucoma (Figure 3-1).

\section{Methods}

\section{Human donor eyes}

Human donor eyes were collected in accordance with the tenets of the Declaration of Helsinki and approved by University of Tennessee Health Science Center (UTHSC) Institutional Review Board (IRB). A human donor eye with a clinical diagnosis of glaucoma (age 67 years; female) and an eye from an age-matched control (with no

\footnotetext{
* Reprinted with permission from John Wiley \& Sons. Chintalapudi SR, Morales-Tirado VM, Williams RW, Jablonski MM. Multipronged approach to identify and validate a novel upstream regulator of Sncg in mouse retinal ganglion cells. FEBS J. 2016 Feb;283(4):678-93. doi: 10.1111/febs.13620. Epub 2016 Jan 18. PubMed PMID: 26663874.
} 


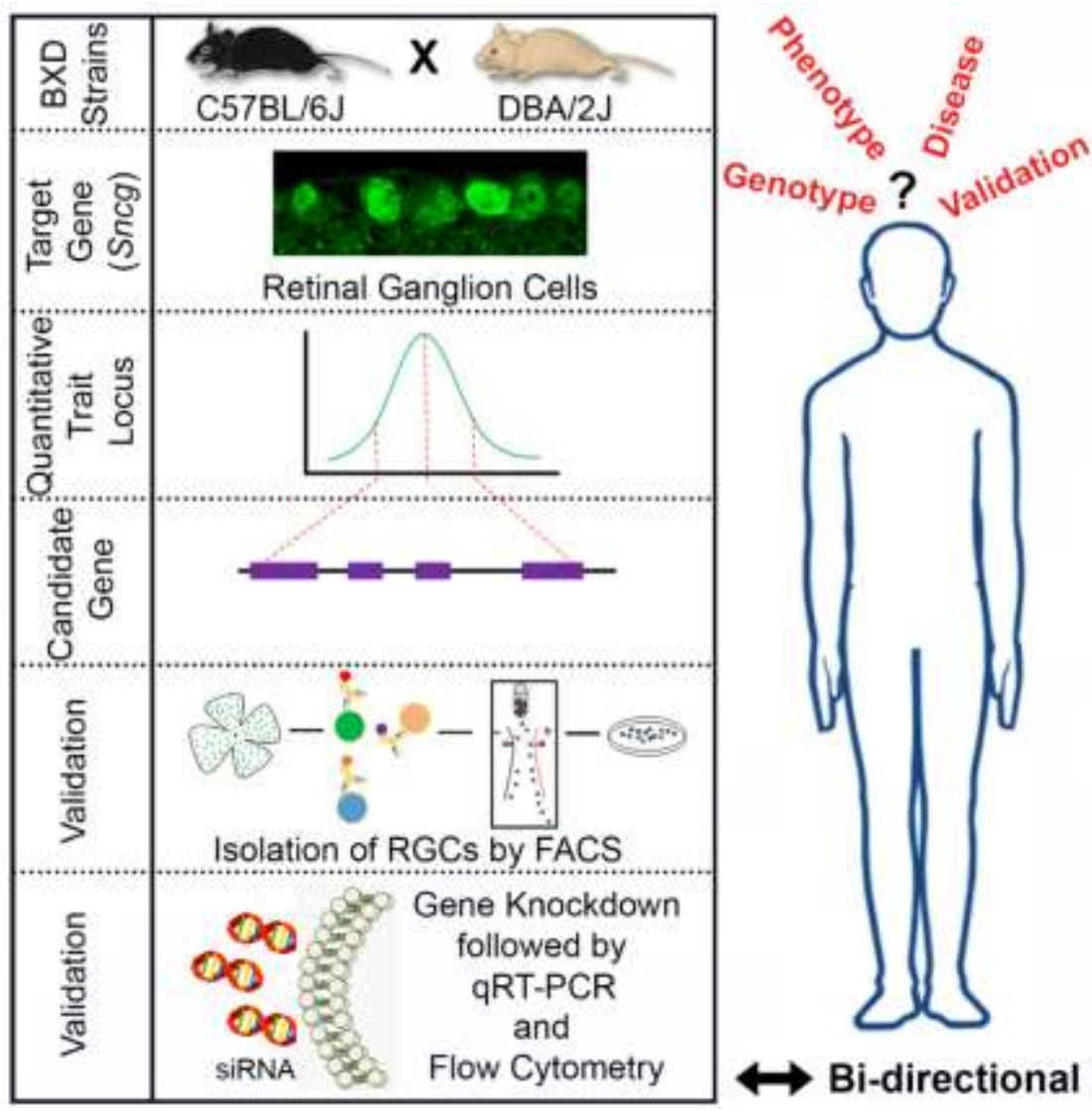

Figure 3-1. Illustrated summary

A combined study using systems genetics with flow cytometry and gene silencing to identify and verify the genetic regulation of Sncg in primary RGCs. This study provides clues to better understanding of disease mechanisms in glaucoma. 
history of eye disease; age 83 years; male) were obtained from the Mid South Eye Bank and the Lions Eye Bank of Oregon in compliance with the Eye Bank Association of America's (EBAA) Medical Standards and Government Regulation. Both donors were free of sepsis and potentially transmissible disease. There was no evidence of high-risk behavior, HIV or hepatitis. Donor eyes were fixed in formalin within five hours of enucleation. The anterior segment structure was removed from both eyes and the fundi were photographed. Full thickness biopsies of the temporal retina of each eye were removed with a 5-mm diameter punch (Miltex, PA, USA). Retinae were carefully dissected away from the retinal pigment epithelium, choriocapillaris and sclera.

\section{BXD strains}

A total of 73 strains of mice at approximately 8 weeks of age, including 69 BXD RI strains, parents and F1 crosses were used in this study. Both sexes were equally represented (117). All procedures involving mice were approved by the Animal Care and Use review board of UTHSC and followed the ARVO Statement for the Use of Animals in Ophthalmic and Vision Research in addition to the guidelines for laboratory animal experiments (Institute of Laboratory Animal Resources, Public Health Service Policy on Humane Care and Use of Laboratory Animals). Animals were housed under cyclic light (12 hour on: 12 hour off) with $35 \%$ humidity in a specific pathogen free environment at UTHSC. In our immunohistochemical analyses, a female BXD66 mouse aged 12 months are used as a model of glaucoma due to their elevated IOP $(18.50 \pm 1.47 \mathrm{mmHg}$; unpublished observation but available on www.GeneNetwork.org) and high degree of optic nerve damage inclusive of many degenerating axons and gliotic scarring that are apparent on cross sections (unpublished observation but available on www.GeneNetwork.org).

\section{HEI Retina Database}

The HEI Retina Database contains the data analysis of 346 Illumina Sentrix ${ }^{\circledR}$ Mouse Whole Genome-6 version 2.0 arrays (Illumina, San Diego, CA). This database contains the retinal transcriptome profiles of 80 strains of BXD mice aged between 2 and 4 months (117). We used the 'Normal HEI Retina Dataset' that is available on GeneNetwork (www.genenetwork.org) to perform QTL analysis and define genetic networks. This data is globally normalized to produce arrays that have a mean of 8 and a standard deviation of $2.0(70,71)$. The GEO Dataset generated by Dr. Simon John and colleagues at the Jackson Laboratory (NCBI accession number GSE26299; Affymetrix platform; http://www.ncbi.nlm.nih.gov/geo/query/acc.cgi?acc=GSE26299) was normalized in the identical manner and uploaded onto GeneNetwork. This dataset was derived from retinae of $\mathrm{DBA} / 2 \mathrm{~J}$ mice with varying degrees of glaucomatous optic nerve damage (38). 


\section{eQTL mapping and heritability calculation}

Simple interval mapping was carried out using the WebQTL module on GeneNetwork to identify any significant eQTL(s) that modulate Sncg expression. Genome wide significance levels were estimated by performing 2000 permutations. To determine how much of the variation in Sncg expression across the cohort was due to genetic effects, we calculated the heritability of Sncg using our published methods (59, 69, 82) \{Lu, 2011 \#261;Lu, 2011 \#204;Swaminathan, 2013 \#100\}.

\section{Identification of candidate gene(s)}

The partial correlation feature on GeneNetwork was used to identify any candidate gene that modulates Sncg expression in retina. A partial correlation is defined as the relationship between a primary variable and a target variable, while controlling for one or more other variables (120). In this case, the correlation between the expression of Sncg (primary variable) and the expression of candidate regulatory gene within the retina database (target variables) was measured after mathematically controlling for the markers—rs8242766 (Chr1 at 172.981863) and rs4136041 (Chr1 at 177.366982) - that straddle the trans-eQTL that was identified using simple interval mapping. By holding constant the genetic variation of this region, any residual biological variation more accurately reflects the correlation between the expression of the Sncg and the candidate regulatory gene(s). In essence, genes with false positive correlations are removed from the list of potential candidate modifiers.

\section{Immunohistochemistry}

Human and murine retinal sections embedded in low melting point agarose were prepared following our published methods (144). Briefly, tissue sections were blocked with $10 \%$ goat serum and permeabilized with $0.1 \%$ Triton X-100. Anti-prefoldin 2 (PFDN2; goat polyclonal IgG, Catalog number: sc-19834; Santa Cruz Biotechnology) and anti- gamma synuclein (SNCG; rabbit polyclonal IgG, Catalog number: GTX110483; GeneTex) were used per manufacturers conditions. Appropriate Alexa Fluor-tagged secondary antibodies (1:200; Invitrogen) and TO-PRO3 iodide (1:4000; Invitrogen) were used to indicate the presence of the antigens of interest and nuclei, respectively. Sections were viewed and images were obtained using a Nikon $\mathrm{C} 1$ confocal microscope within the Imaging Core Facility in the Hamilton Eye Institute. All microscope settings, including laser levels and gain, were held constant to allow for relative comparisons of signal intensity within and between experiments. 


\section{Enrichment of primary murine RCGs}

\section{Cell suspension of retinae cells}

C57BL/6J mice ( 80 mice at 5-8 weeks of age) were sacrificed by cervical dislocation followed by enucleation. Collected retinae were dissociated using enzymatic digestion containing papain (15 IU/mL papain, $5 \mathrm{mM}$ L-cysteine and 200U/mL DNase I) in PBS. Enzymes were inactivated by the addition of PBS $/ 10 \%$ fetal bovine serum (FBS; Hyclone Laboratories, Inc.). The cell suspension was filtered through a Falcon $70 \mu \mathrm{M}$ nylon strainer (BD Biosciences, San Jose, CA) and centrifuged at 1300 RPM for 5 minutes at RT. Cells were resuspended in PBS/1\% FBS and kept on ice until ready to use. Cell viability was assessed by trypan blue exclusion (145).

\section{Cell labeling}

We used a 1:100 solution of an amine-reactive dye, Zombie Aqua ${ }^{\mathrm{TM}}$ (BioLegend), diluted in PBS to discriminate between live and dead cells. Prior to immunolabeling, cells were treated with $5 \mu \mathrm{L}$ of anti-CD16/32 (Fc $\gamma \mathrm{R}$ II/III block, BioLegend) to minimize nonspecific binding of antibodies. CD16/32 are also known markers for monocytes and macrophages. Aliquot of unlabeled retinal cells were used as negative control to determine the degree of autofluorescence and to control for voltages. The following isotype controls were used to confirm the specificity of primary and secondary antibodies: mouse IgG1 (clone MOPC-21, BioLegend) PE-Cy7, AF700, FITC PerCPCy5.5 and rat IgG2b (clone RTK4530, BioLegend). The AbC ${ }^{\text {TM }}$ Anti-Mouse Bead Kit (Life Technologies, Carlsbad, $\underline{\mathrm{CA}}$ ) was used as an instrument calibration and singlefluorochrome reference. The following primary antibodies were used to label cells for 30 minutes on ice: anti-CD90.1 PerCP-Cy5.5 (Thy1.1, clone OX-7, Catalog number: 202516, BioLegend; exhibits no cross-reactivity with CD90.2) and anti-CD90.2 Alexa Fluor-700 (Thy1.2, clone 30-H12, Catalog number: 105320, BioLegend; exhibits no cross-reactivity with CD90.1) as a Pan-Thy1 marker; and anti-CD48 PE-Cy7 (clone HM48-1, Catalog number: 103424, BioLegend). Cells were enriched by FACS using a BD Biosciences FACSAria Cell Sorter (BD Biosciences).

\section{Sorting strategy}

Live cells were gated by Zombie Aqua ${ }^{\mathrm{TM}}$ exclusion followed by Pan-Thy1 positivity. Live PanThy $1^{+}$cells were selected based upon negative CD48 expression. The collected population of RGCs had the following phenotype: Live PanThy $1^{+} \mathrm{CD} 48^{\text {neg }}$ cells.

\section{Flow cytometry analyses}

\section{Intracellular straining}

Cells were fixed for $1 \mathrm{hr}$ at $4^{\circ} \mathrm{C}$ using BD Cytofix/ Cytoperm ${ }^{\mathrm{TM}}$ Buffer (BD Biosciences). Anti-gamma synuclein (dilution: 1:100; GeneTex Inc.) and anti-prefoldin 2 
(dilution: 1:100; Santa Cruz Biotechnology, San Diego, CA) antibodies were diluted in permeabilization buffer and incubated with cells for $1 \mathrm{hr}$ at $4^{\circ} \mathrm{C}$. Alexa Fluor-568 goat anti-rabbit IgG or Alexa Fluor-488 donkey anti-goat IgG (Molecular Probes) were used as secondary antibodies. Cells were kept in PBS/1\%FBS until ready for analysis. Data acquisition was performed on a BD LSRII Flow Cytometer (BD Biosciences) and analyses were performed using FlowJo vX10.0.6 (Tree Star, Inc., Ashland, OR).

\section{Cell proliferation studies}

Sorted, enriched-primary RGCs were kept either for 4 hours or overnight at $4{ }^{\circ} \mathrm{C}$ to restore the integrity of the cell membrane to ensure that cells were healthy prior to further treatments. Enriched RGCs were re-suspended in PBS at a concentration of $5 \times 10^{6}$ cells $/ \mathrm{mL}$ and labeled with $1 \mu \mathrm{M} \mathrm{CFSE}$ in PBS $/ 1 \%$ FBS for $5 \mathrm{~min}$ at $37^{\circ} \mathrm{C}(146)$. Cells were quenched three times for 10 minutes on ice bucket in $20 \mathrm{~mL}$ volume with cold media-containing $10 \%$ serum. Labeled enriched RGCs $\left(1 \times 10^{5}\right.$ per well) were cultured in 96 -well bottom plates for 48 hours at $37^{\circ} \mathrm{C}$. At 0 and 48 hours, cells were harvested, washed and analyzed. Acquisition proceeded using a BD LSRII Flow Cytometer equipped with 4-lasers (BD Biosciences, San Jose) and analyses were performed using FlowJo software (Tree Star, Inc., Ashland, OR), Cell Proliferation application. A reduction in median fluorescent intensity (MFI) is indicative of mitotic activity.

\section{RGC culture}

The culture conditions developed Alissa Winzeler and Jack T. Wang (147) was followed for the maintenance of sorted mouse RGCs in monolayer culture in a 96 well plate. The cells were cultured in absence of serum, as the factors in serum are not well defined and can vary widely between sample batches. Also, presence of serum in culture has the potential to alter cellular responses dramatically. The medium contained Dulbecco's modified Eagle's medium, Neurobasal medium, plus insulin $(5 \mu \mathrm{g} / \mathrm{mL})$, progesterone sodium pyruvate $(110 \mu \mathrm{g} / \mathrm{mL})$, penicillin $(100 \mathrm{U} / \mathrm{mL})$, streptomycin $(100 \mathrm{U} / \mathrm{mL})$, thyroxine (T3; $40 \mathrm{ng} / \mathrm{mL})$, L-glutamine $(292 \mu \mathrm{g} / \mathrm{mL})$, NS21 supplement (1X), N-acetyl-L-cysteine (NAC; $5 \mu \mathrm{g} / \mathrm{mL}$ ). Additional serum-free supplements were added to further promote optimal RGC survival, this included Bottenstein-Sato supplement (SATO; 1X). SATO was prepared by combining BSA $(10 \mathrm{mg} / \mathrm{mL})$, transferrin $(10 \mathrm{mg} / \mathrm{mL})$, putrescine $(1.6 \mathrm{mg} / \mathrm{mL})$, progesterone $(6 \mu \mathrm{g} / \mathrm{mL})$, sodium selenite $(4 \mu \mathrm{g} / \mathrm{mL})$. Additional trophic factors were also added to promote RGC health and survival, these include- brain derived growth factor (BDNF; $50 \mu \mathrm{g} / \mathrm{mL}$ ), ciliary neurotrophic factor $(\mathrm{CNTF} ; 10 \mu \mathrm{g} / \mathrm{mL})$ and forskolin $(4.2 \mathrm{mg} / \mathrm{mL})$. Seeding densities ranged from $40,000-60,000$ cells/well. Cultures were incubated at $37^{\circ} \mathrm{C}$ in a humidified atmosphere containing $10 \% \mathrm{CO} 2$ and $90 \%$ air. 


\section{siRNA transfection in primary RGCs}

Mitotically active RGCs were cultured in 96 well plates for $24 \mathrm{hrs}$ in RGC culture media (147) after which the media was replaced with Accell delivery media (Dharmacon) containing SMARTpool siRNA targeting mouse $P f d n 2$, a pool of 4 different siRNAs targeting the gene to increase the potency (Target sequence 1:

GCAAAGAACUGAACGAAUU, Target sequence 2: UGAUUAAAUGUUUUGGUCA, Target sequence 3: GAUUCCCACUUGUAAUUUC, Target sequence 4:

GGACUGUCAAAGAAGUGCU; Cat no: E-062703-00-0005; Dharmacon) or a nontargeting GFP fluorescent siRNA (Cat no: D-001950-01-05; Dharmacon) at a final concentration of $1 \mu \mathrm{M}$ according to the manufacturer's protocol. Transfected cells were cultured at $37^{\circ} \mathrm{C}$ for $48 \mathrm{hrs}$. Assessment of live cells after siRNA transfection was performed by labeling cells with a 1:100 solution of an amine-reactive dye, Zombie $\operatorname{Red}^{\mathrm{TM}}$ (BioLegend) diluted in PBS to avoid spectral overlap with GFP (emission: emission $624 \mathrm{~nm}$ ). Cells were incubated for 20 minutes at RT followed by two washes. Finally, cells were suspended in PBS/1\%FBS prior to analysis.

\section{RNA isolation, cDNA synthesis and quantitative polymerase chain reaction}

\section{RNA isolation}

RNA from $1.0 \times 10^{6}$ retinae cells was extracted using the Qiagen ${ }^{\circledR}$ miRNeasy Mini Kit (Qiagen, Valencia, CA) per manufacturer's protocol. Briefly, cells were lysed by using a pipette, prior addition of chloroform and a series of alcohol induced precipitation. The extract was passed through a spin column followed by on-column DNase digestion to increase purity and yield. RNA purity and concentration was assessed by analysis on NanoDrop Spectrophotometer. RNA used for cDNA synthesis met the following requirements: A260:A230 ratio greater than 1.7; A260:A280 ratio between 1.8 to 2.1 .

\section{cDNA synthesis}

Genomic DNA elimination and First-Strand cDNA synthesis were performed using SuperScript ${ }^{\circledR}$ VILO ${ }^{\mathrm{TM}}$ cDNA Synthesis Kit (Life Technologies). Briefly, $70 \mathrm{ng}$ of RNA were combined with VILO ${ }^{\mathrm{TM}}$ Reaction Mix and Enzyme Mix using the following conditions: $25^{\circ} \mathrm{C}$ for 10 minutes, $42^{\circ} \mathrm{C}$ for $60 \mathrm{~min}$ and $85^{\circ} \mathrm{C}$ for 5 minutes. For preamplification of cDNA, the RT product was pre-amplified for $S n c g$ and $P f d n 2$ genes using TaqMan ${ }^{\circledR}$ Gene Expression Assays and TaqMan ${ }^{\circledR}$ PreAmp Master Mix as per manufacturer's protocols (Life Technologies Inc., Applied Biosystems). The preamplified cDNA was diluted with 1x TE (Tris/EDTA) at a 1:5 ratios and $2.5 \mu 1$ of the cDNA was used in each well for qRT-PCR reactions. 


\section{$q R T-P C R$ reaction}

The following amplicons were used for the qRT-PCR reaction: Sncg, Mm00488345_m1; Pfdn2, Mm00448103_m1; and Gapdh, Mm99999915_g1 (Life Technologies). The ready-to-use TaqMan ${ }^{\bar{B}}$ Universal Master Mix II with UNG (Applied Biosystems) contained AmpliTaq Gold ${ }^{\circledR}$ DNA Polymerase, dNTPs , ROX ${ }^{\mathrm{TM}}$ Passive Reference, optimized buffer components. Reactions were run on a Roche LightCycler ${ }^{\circledR}$ 480 Real-Time PCR system (Roche, Indianapolis, IN) in 96-well plate format with a 10 $\mu \mathrm{L}$ final reaction volume at Molecular Resource Center at UTHSC. All reactions were performed in triplicates from three independent biological replicates. Relative quantitation was performed using the comparative threshold $\left(\mathrm{C}_{\mathrm{T}}\right)$ after determining the values of $\mathrm{C}_{\mathrm{T}}$ for the reference gene Gapdh and the target gene Sncg or Pfdn2 in each sample. $R_{\mathrm{q}}$ was calculated using Equation 3.1:

$$
R_{\mathrm{q}}=2^{-\Delta \Delta \mathrm{C}_{\mathrm{T}}}
$$

$\Delta \mathrm{C}_{\mathrm{T}}=\left(\mathrm{C}_{\mathrm{T}}\right.$ target gene $)-\left(\mathrm{C}_{\mathrm{T}}\right.$ reference gene $)$

$\Delta \Delta \mathrm{C}_{\mathrm{T}}=\left(\Delta \mathrm{C}_{\mathrm{T}}\right.$ treatment sample $)-\left(\Delta \mathrm{C}_{\mathrm{T}}\right.$ reference control sample $)$

\section{Statistics}

Data presented as mean \pm SEM. One-way ANOVA with Tukey's post hoc tests (PRISM GraphPad) were used to determine statistical significance. Differences were considered significant at $p<0.001$.

\section{Correlation comparison and gene ontology tree machine construction}

A secondary goal of our investigation was to identify genes that were co-regulated with $S n c g$ and $P f d n 2$ and shared a functional relationship. As a first step in this process, the transcript levels of our genes of interest—Sncg and Pfdn2-were compared using Pearson correlation with all 45,281 probe sets present on the Illumina V6.2 array. The top 500 genetically correlated genes in the retinal database were selected. To produce a set of shared correlated transcripts, we selected all common transcripts of Sncg and Pfdn2 within the list of the top 500 correlates of both genes. After removing the transcripts with genes with expression levels less than 7 based on the expression data normalization process. The remaining list of 163 common correlates were analyzed by Gene Ontology enrichment analysis WebGestalt (http://bioinfo.vanderbilt.edu/webgestalt), as described previously $(69,82)$. GO enrichment analysis allows users to input lists of highly correlated genes through the web interface, identifies GO terms that are significantly associated with the input gene lists, and visualizes the enriched GO terms in a directed acyclic graph (DAG). 


\section{Results}

\section{Identification of $P f d n 2$ as a candidate gene modulating $S n c g$ expression in murine RGCs}

To identify the genomic regions that modulate Sncg expression in the retina, we used a systems genetics approach using the Illumina Array data from murine retinae. On the Illumina arrays, Sncg is represented by two probe sets_-ILMN_2939277 and ILMN_2598478 - that hybridize to nucleotides within exon 4 and 1, respectively. Both probe sets map with significant likelihood ratio statistic (LRS) scores to the same location on the minus strand on Chr 14 at $35.187676 \mathrm{Mb}$. Because the ILMN_2598478 probe set reflected higher average expression levels, it was selected to represent Sncg in our analyses. The expression of Sncg in the retina among BXD mice varied (Figure 3-2) with BXD15 having the lowest Sncg gene expression of 12.65 \pm 0.03 (expression $\log _{2} \pm$ SEM) and BXD61 having the highest at $14.47 \pm 0.10$. The average expression among all BXDs was 13.80 \pm 0.33 . The parental lines, C57BL/6J and DBA/2J, had Sncg expression levels of 13.75 \pm 0.15 and 14.27 \pm 0.09 , respectively at 1-2 months of age (Figure 3-2; Appendix B, Table B-1). The heritability of the variation in $S n c g$ expression was 0.57 . This result demonstrates that $57 \%$ of the variation in $S n c g$ expression was due to genetic effects; and the remaining $43 \%$ was due to environmental influences. The six SNPs and two deletions in Sncg (Table 3-1) likely contribute to the genetic component of the heritability. The simple interval map shows a significant trans-eQTL for Sncg on distal chromosome 1 (Figure 3-3). The maximum LRS score of the locus was $34.1 \mathrm{Mb}$, which is equivalent to a LOD ratio of 7.40. The confidence interval of this strong QTL extends from 171.5 to 183.5 Mb (Figure 3-3 inset).

To identify the candidate gene(s) that modulates the expression of Sncg in the retina, we performed partial correlation analysis within GeneNetwork (Figure 3-4). A partial correlation reflects the level of association between a primary variable (i.e., $S n c g$ expression level) and a target variable (i.e., upstream regulator of Sncg expression level) after controlling for one or more variables (i.e., the genetic variability of the trans-eQTL peak on Chr 1 (120). In our study, applying partial correlation analysis allowed us to mathematically control for the LRS peak on Chrl and identified a single gene candidate, $P f d n 2$ (partial Pearson correlation value: $\mathrm{r}=0.656 ; \mathrm{p}=3.73 \times 10^{-13}$ ). No other genes in that interval had significant expression levels and significant correlation values. This outcome solidified $P f d n 2$ as the candidate upstream modulator of $S n c g$. On the Illumina array, $P f d n 2$ is represented by one probe set_-ILMN_129667_that hybridizes to exon 4 (Chr1 at $173.286888 \mathrm{Mb}$ on the plus strand). The expression level of Pfdn2 varied among the BXD strains from a low of $12.49 \pm 0.10$ in BXD13 to a high of $14.41 \pm 0.2$ in BXD39, with an average expression of $13.45 \pm 0.08$ (Figure 3-5). The parental lines, C57BL/6J and DBA/2J, had $P f d n 2$ expression levels of $13.63 \pm 0.21$ and $13.84 \pm 0.23$, respectively at $1-2$ months of age (Appendix B, Table B-2). The heritability of the variation in $P f d n 2$ expression was $39 \%$ and the remaining $61 \%$ of the variation in the expression was due to environmental influences. To determine if there was any association between Sncg and

$P f d n 2$ in healthy young adult mice eyes to performed Pearson correlation analysis. The 


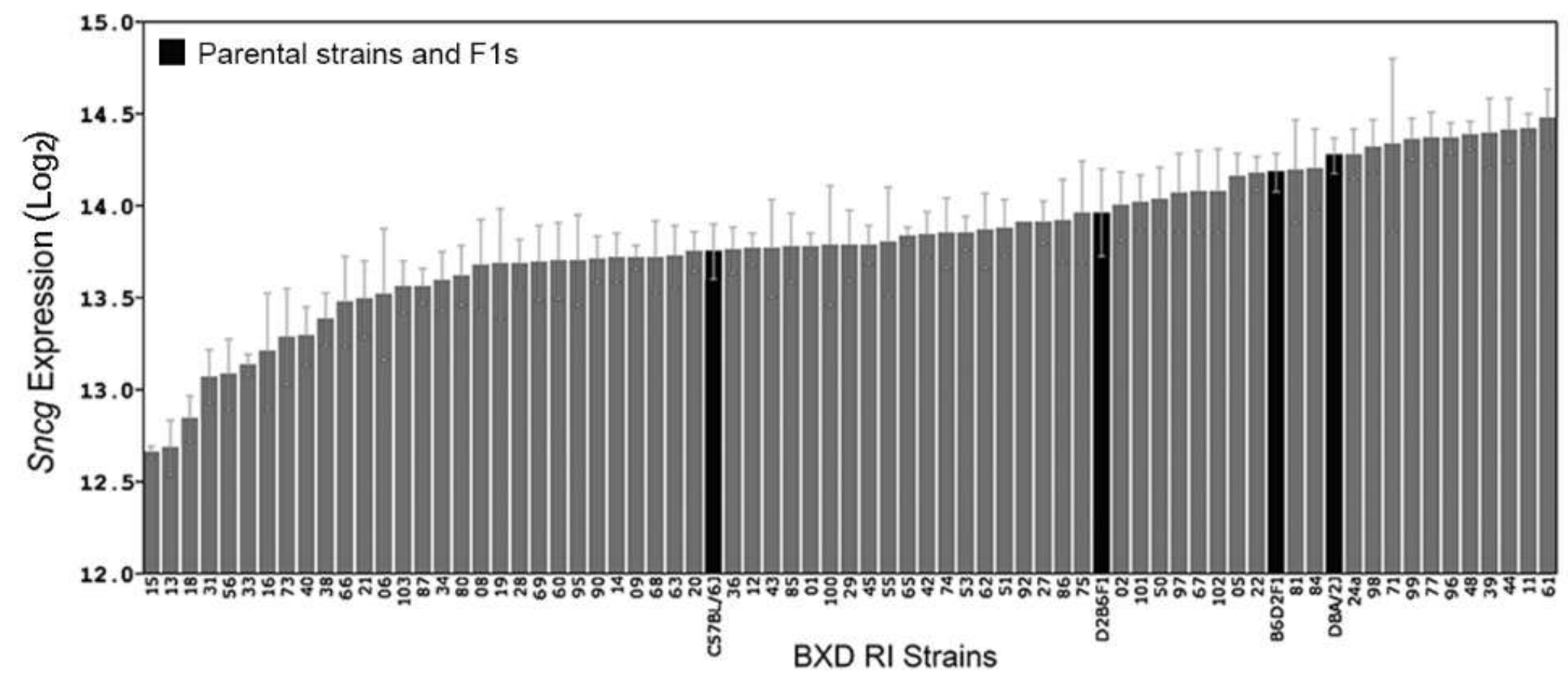

Figure 3-2. mRNA expression levels of Sncg across BXD strains and parental strains

Rank-ordered mean Sncg mRNA levels across the BXD recombinant inbred family. The expression of Sncg in the retina among BXD mice varied with BXD15 having the lowest Sncg gene expression of $12.65 \pm 0.03$ (expression $\log _{2} \pm$ SEM) and BXD61 having the highest at $14.47 \pm 0.10$. The BXD strains and parental strains are depicted in grey and black, respectively. Values denote normalized relative mRNA expression levels on $\log _{2}$ scale (mean \pm SEM). 
Table 3-1. Summary of candidate genes Sncg and Pfdn2

\begin{tabular}{|c|c|c|c|c|c|c|}
\hline $\begin{array}{c}\text { Gene } \\
\text { I.D. }\end{array}$ & Location & $\begin{array}{c}\text { Max LRS Value } \\
\text { and } \\
\text { Location }\end{array}$ & $\begin{array}{l}\text { Probe set I.D. and } \\
\text { BLAT Specificity }\end{array}$ & $\begin{array}{l}\text { Markers } \\
\text { Flanking } \\
\text { the Gene }\end{array}$ & $\begin{array}{c}\text { Mean } \\
\text { Expression } \\
\text { Level } \\
\end{array}$ & SNPs and Indels \\
\hline Sncg & $\begin{array}{l}\text { Chr14@ } \\
35.187676 \mathrm{Mb}\end{array}$ & $\begin{array}{l}34.3 ; \\
\text { Chr1:174.792334 }\end{array}$ & $\begin{array}{l}\text { ILMN_2598478 } \\
; 2.5\end{array}$ & $\begin{array}{l}\text { rs13482135@ } \\
36.200742 \mathrm{Mb}\end{array}$ & 13.804 & $\begin{array}{l}\text { - Six intronic SNPs } \\
\text { - Two deletions }\end{array}$ \\
\hline$P f d n 2$ & $\begin{array}{l}\text { Chr1@ } \\
\text { 173.286888 Mb }\end{array}$ & $\begin{array}{l}\text { 12.8; } \\
\text { Chr5:85.890222 }\end{array}$ & $\begin{array}{l}\text { ILMN_1219667 } \\
; 3.1\end{array}$ & $\begin{array}{l}\text { rs3722740@ } \\
173.679247 \mathrm{Mb}\end{array}$ & 12.8 & $\begin{array}{l}\text { - } 48 \text { intronic SNPs } \\
\text { - One 3’UTR SNP } \\
\text { - One synonymous SNP } \\
\text { in exon } 4 \\
(\mathrm{~A} \rightarrow \mathrm{A}, \mathrm{gcA} \rightarrow \mathrm{gcG}) \\
\text { - Four insertions } \\
\text { - Six deletions }\end{array}$ \\
\hline
\end{tabular}




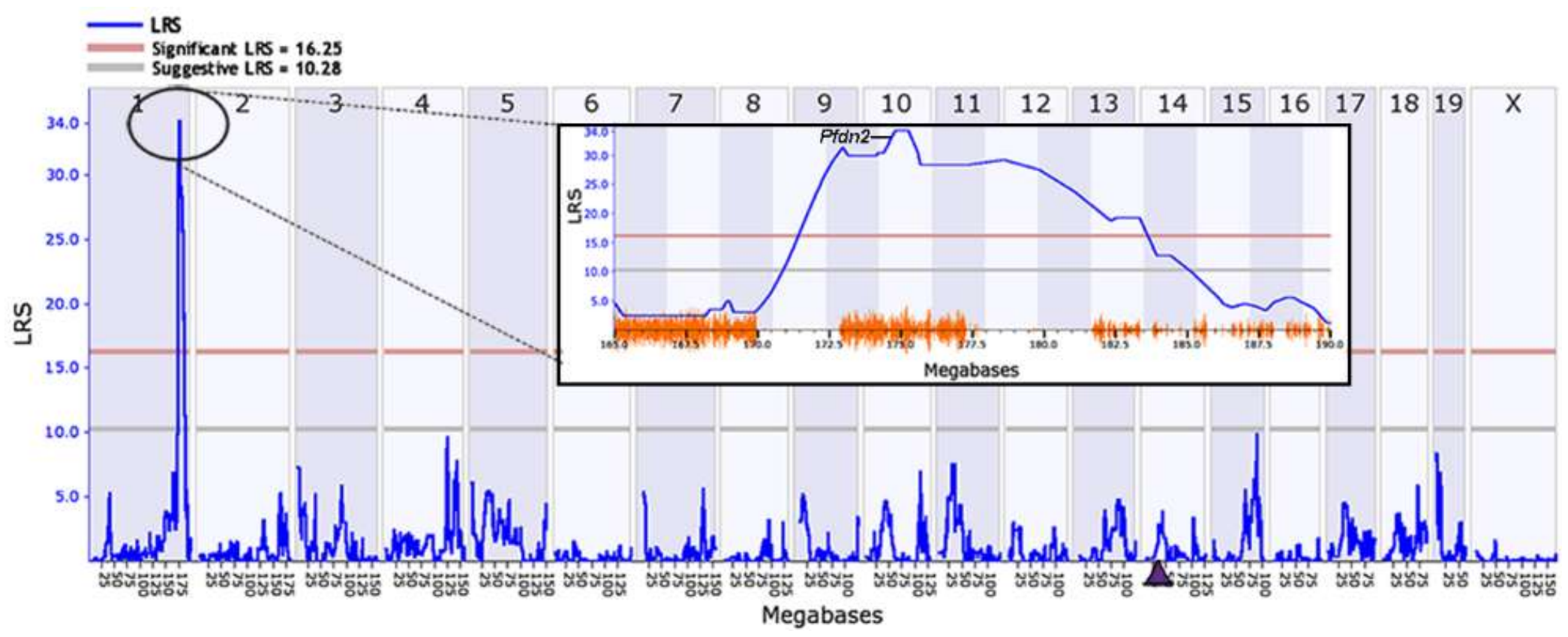

Figure 3-3. QTL mapping of Sncg

A significant trans-eQTL for Sncg is present on chromosome 1 at 167-190 Mb. Red and gray horizontal lines dictate significant and suggestive thresholds, respectively. The purple triangle indicates the location of Sncg within the genome. The insert depicts a zoomedin view of the trans-eQTL on Chr1, which shows the location of Pfdn2 within the LRS peak. 


\section{GeneNetwork}

University of Tennessee: www.genenetwork.org

Use GeneNetwork 2

I Home I Search I Help | News I References | Policies I Links I

Please select one primary trait, one to three control traits, and at least one target trait.

\begin{tabular}{|c|c|c|c|c|c|c|c|c|c|c|c|c|}
\hline Index & Primary $(x)$ & Control (Z) & Target (r) & Ignored & $\begin{array}{l}\text { Dataset } \\
\text { as }\end{array}$ & $\begin{array}{l}\text { Trait } \\
\text { ID } \\
80\end{array}$ & $\begin{array}{l}\text { Description } \\
\text { ERe }\end{array}$ & $\begin{array}{l}\text { Location } \\
\text { ne }\end{array}$ & $\begin{array}{l}\text { Mean } \\
\text { Ee }\end{array}$ & $\begin{array}{l}\text { N } \\
\text { Cases } \\
\text { Sp }\end{array}$ & $\begin{array}{l}\text { Max LRS } \\
\text { ae }\end{array}$ & $\begin{array}{l}\text { Max LRS Location } \\
\text { Chr and Mb } \\
\text { Ce }\end{array}$ \\
\hline 1 & 0 & - & 0 & 0 & BXoGeno & F58242766 & - & Chr1: 172.981863 & - & 93 & - & - \\
\hline 2 & 0 & - & 0 & 0 & BXDGeno & rs4136041 & - & Chr1: 177.366982 & - & 92 & - & - \\
\hline 3 & - & 0 & ○ & 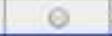 & G2NEI_ILM_Retina_BXD_RL0410 & ILMN_2598478 & synudein, gamma; exon 1 & Chr14: 35.187676 & 13.804 & 74 & 34.3 & Chr1: 174,792334 \\
\hline
\end{tabular}

Compute partial correlation for target selected above:

Pearson's $r$ Spearman's rto

\section{Figure 3-4. Partial correlation analysis}

The screen shot depicts the partial correlation analysis using the tools available on GN. Select and add Sncg into the BXD Trait

Collection in addition to two SNP markers rs8242766 and rs4136041, which are located at the borders of the QTL on Chr 1 between 172 and $178 \mathrm{Mb}$ (the location of the trans-eQTLs). 


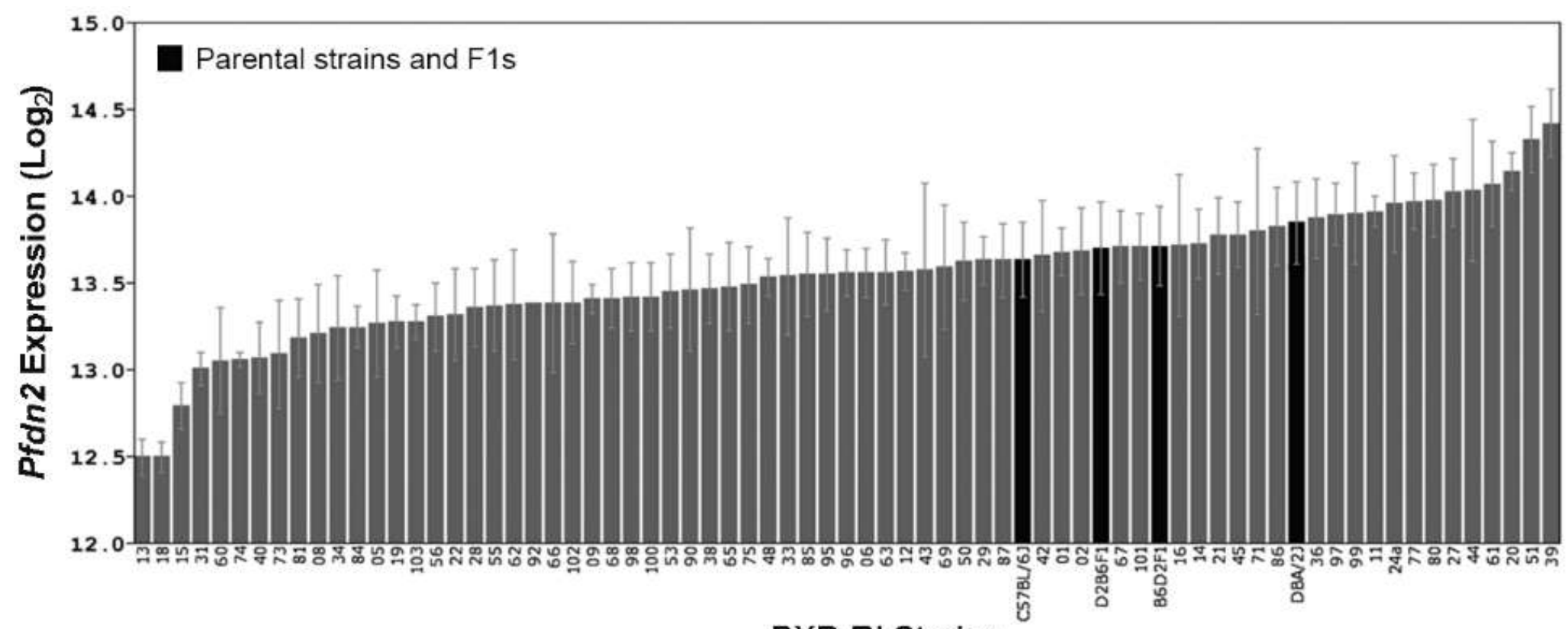

BXD RI Strains

\section{Figure 3-5. Expression levels of $P f d n 2$ across BXD strains and parental strains}

Rank-ordered mean $P f d n 2$ expression levels across the BXD family of mice. The expression level of $P f d n 2$ varied among the BXD strains from a low of $12.49 \pm 0.10$ in BXD13 to a high of $14.41 \pm 0.2$ in BXD39, with an average expression of $13.45 \pm 0.08$. The BXD strains and parental strains are depicted in grey and black, respectively. Values denote normalized relative mRNA expression levels on $\log _{2}$ scale (mean \pm SEM). 
expression levels of $S n c g$ and $P f d n 2$ in the retinae of BXD mice aged 1-2 months were positively correlated (Pearson correlation value: $r=0.609 ; p=3.38 \times 10^{-09}$; Figure 3-6A), which demonstrates that the two genes co-vary in the healthy retina.

As DBA/2J mice age, retinal ganglion loss becomes prominent. To investigate the relationship between $S n c g$ and $P f d n 2$ in aged DBA/2J mice, we used a GEO dataset generated by Howell et al. (38). As noted by the authors, their DBA/2J mouse panel exhibited varying degrees of glaucoma-associated damage at 10.5 months including an increase in IOP and optic nerve head damage. On the Affymetrix panel used by Howell et al., Sncg is represented by one probe set, 1417788_at, which hybridizes to exon 1 of Sncg (Chr 14 at 35.183635 on the minus strand). On the same Affymetrix array, Pfdn2 is represented by two probe sets, 1421950_at and 1435620_at, which hybridize to the last three exons and far 3'UTR, respectively. Because probe set 1421950_at hybridizes to exons rather than a regulatory region, it was used as the single representation of $P f d n 2$ expression in our studies. The average expression levels of $S n c g$ and $P f d n 2$ across the glaucomatous DBA/2J mice were $10.00 \pm 0.85$ and $11.00 \pm 0.22$, respectively. A significant variation in the expression of Sncg was noted among these mice, which ranged between a low of 8.08 in a mouse with a high degree of glaucomatous damage and a high of 10.72 in a mouse with a low degree of optic nerve damage. $P d f n 2$ expression varied in an inverse manner with an expression of 10.62 in a mouse with a low degree of glaucomatous damage to 11.54 in a mouse with a high degree of glaucomatous damage. The direct Pearson correlation between Sncg and Pfdn2 across the D2 panel was significant $\left(\mathrm{r}=-0.819 ; \mathrm{p}=1.09 \times 10^{-14}\right.$; Figure 3-6B).

\section{Subcellular localization of SNCG and PFDN2 demonstrate cytosolic co-localization in mouse and human RGCs}

To compile additional evidence to support our hypothesis that $P f d n 2$ modulates Sncg expression, we determined the subcellular localization of both proteins in retinae from healthy mouse eyes using immunohistochemical analyses (Figure 3-7). SNCG was strongly detected in GCL in the retinas of 3 month old mice indicating that SNCG expression is limited to cells in the GCL (Figure 3-7A, B and C). PFDN2 immunofluorescence was ubiquitous in retina was deteched in NFL, GCL, IPL, OPL and ONL (Figure 3-7D, E and F). The negative controls using only secondary antibody for both SNCG and PFDN2 are shown in Figure 2G-I and 2J-L, we observed no cross reactivity with specific antibodies used in this study. In these investigations, identical procedures including confocal laser settings were maintained throughout to allow for qualitative comparisons between samples. Cross sections of healthy retinae from both mice (Figure 3-8A, B, C and D) and human (Figure 3-8I, J, K and L) illustrate colocalization of SNCG (red) and PFDN2 (green) in the cytoplasm of RGCs and also in the NFL. In contrast, the sub-cellular localization and abundance of SNCG in retinae of glaucomatous BXD66 mice was decreased compared to healthy mice (Figure 3-8E, F, G

and $\mathbf{H}$ ). Similarly, there was an identical reduction in the intensity of SNCG labeling in the retina from a human glaucoma patient (Figure 3-8M, N, $\mathbf{O}$ and $\mathbf{P}$ ) compared to a healthy control (Figure 3-8I, J, K and L). Changes were specific to SNCG 

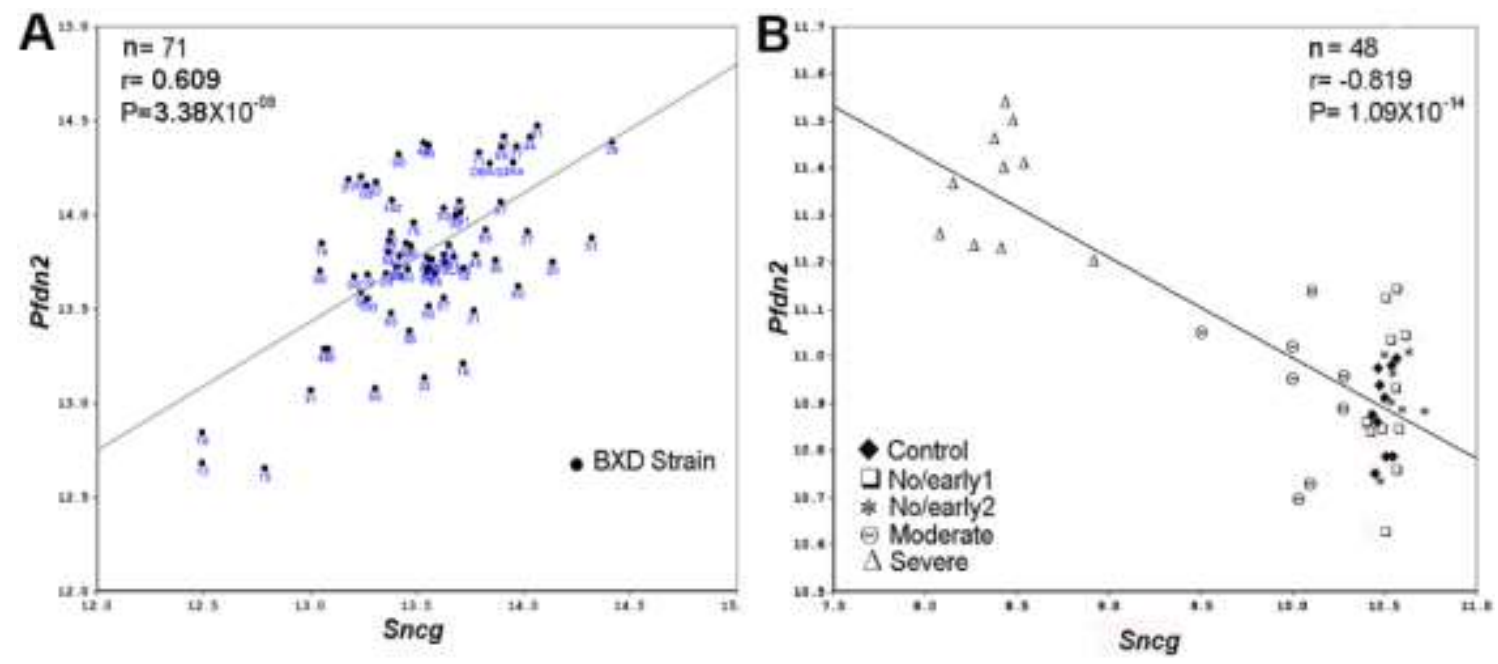

Figure 3-6. Pearson correlation between Sncg and Pfdn 2 expression in retinas of BXD mice

(A) The graph represents a strong positive correlation between expression levels of Sncg and $P f d n 2\left(\mathrm{p}=3.37 \times 10^{-09}\right)$ in the retina of young mice with no glaucomatous damage. Numbers indicate BXD strains, and the parents. (B) The graph represents a strong negative correlation between $S n c g$ and $P f d n 2\left(\mathrm{p}<10^{-14}\right)$ is present in retinae from aged DBA/2J glaucoma mice. Optic nerves from aged DBA/2J mice derived from the Howell et al, data set had the following degrees of axonal damage: Severe (greater than $50 \%$ axons lost, $\Delta$ ); Moderate ( $10 \%-50 \%$ of axons lost, $\Theta$ ); No/early 1 (no detectable damage, *); No/early 2 (no detectable damage, $\square$ ); and Control (no glaucoma; 10.5-month-old $\mathrm{DBA} / 2 \mathrm{~J}-G_{p n m b}{ }^{+} \mathrm{Sj}$ mice, $\bullet$ ). 


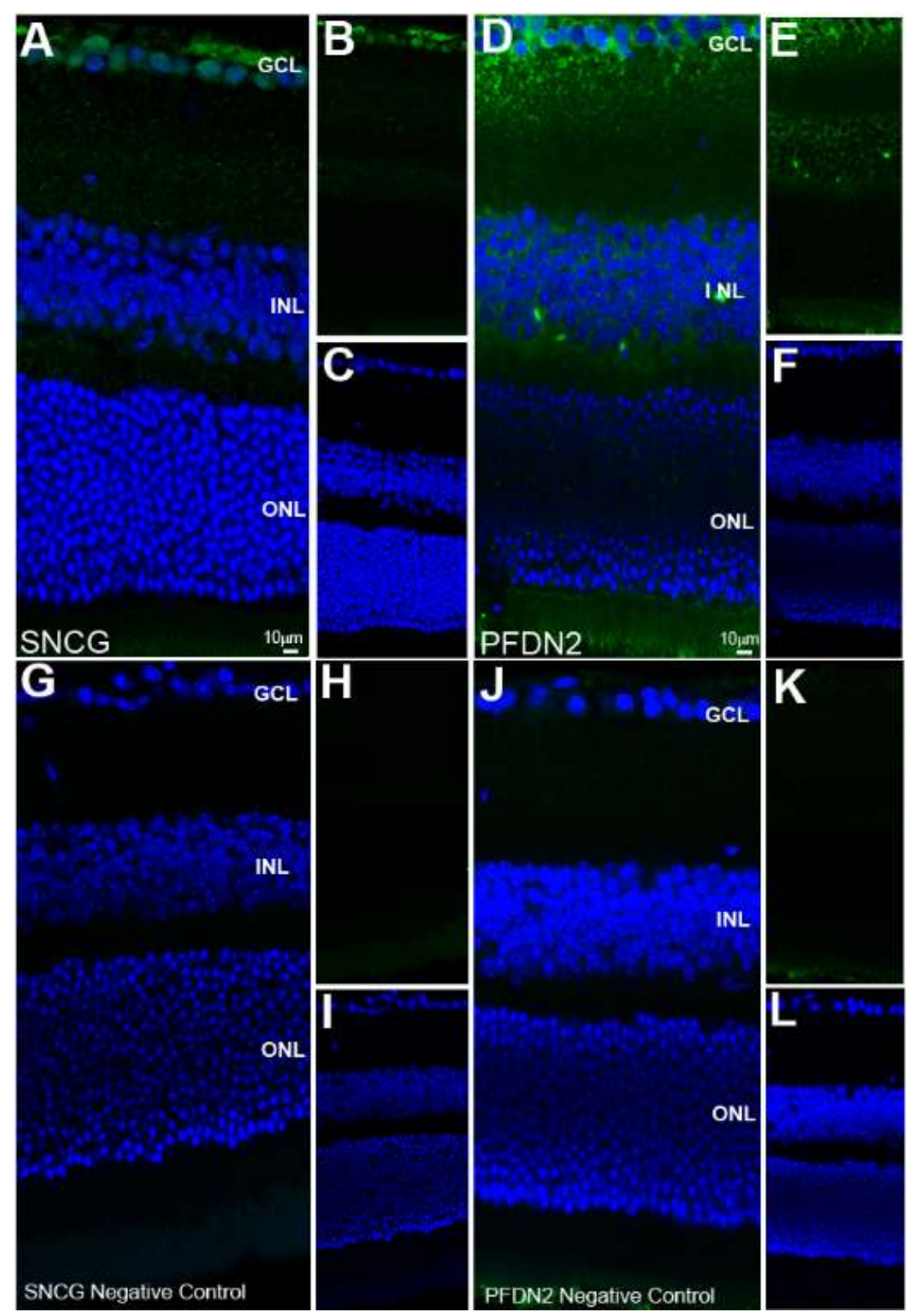

Figure 3-7. Detection of SNCG and PFDN2 in healthy mouse retina

(A-C) In healthy control mouse SNCG (green) immunoreactivity was detected in the GCL. (D-F) PFDN2 (green) was ubiquitously expressed in all the retinal cells. To-PROIII iodide (blue) counterstained the nuclei. Merged images are shown in A and D. (G-I and J-L) Negative controls show no non-specific labeling of the antibodies. NFL= nerve fiber layer, $\mathrm{GCL}=$ ganglion cell layer, $\mathrm{IPL}=$ inner plexiform layer, $\mathrm{INL}=$ inner nuclear layer, $\mathrm{OPL}=$ outer plexiform layer, $\mathrm{ONL}=$ outer nuclear layer. Scale bars: $10 \mu \mathrm{m}$. 

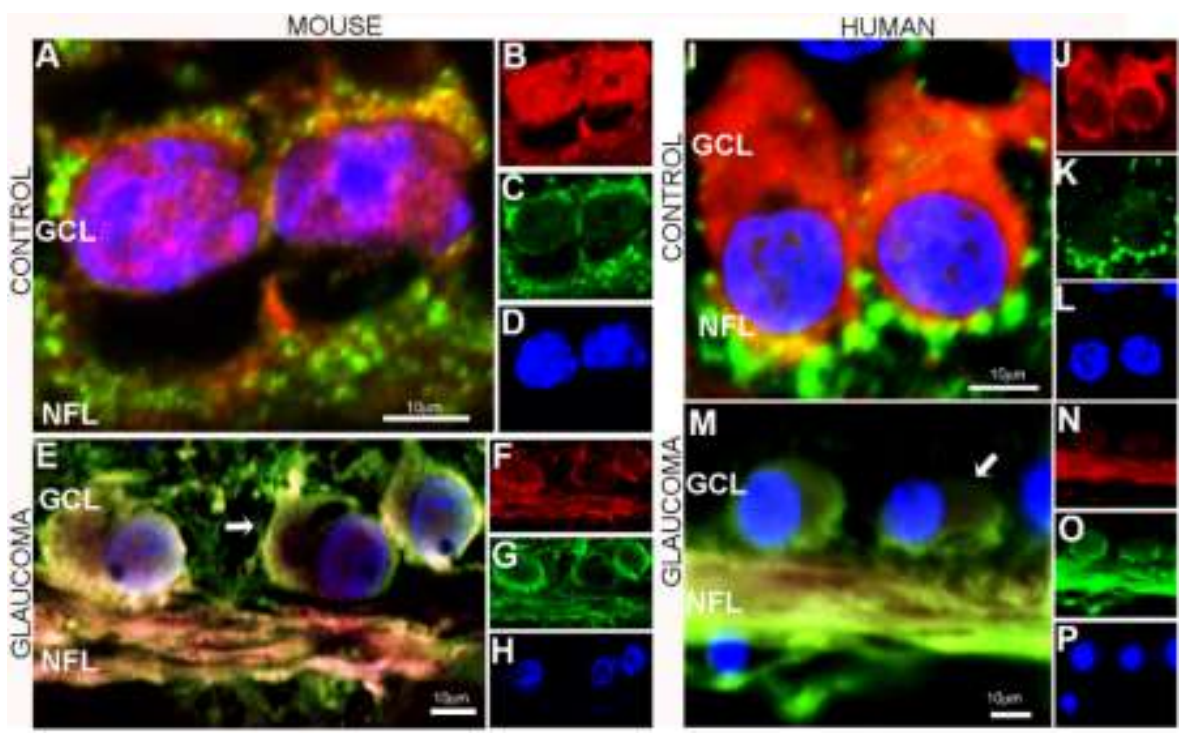

Figure 3-8. Co-localization of SNCG and PFDN2 in healthy and diseased mouse and human RGCs

(A-D) In a control mouse (C57BL/6) and (E-H) a mouse model of glaucoma (BXD66), SNCG (red) was localized throughout the cytoplasm, nuclei and nerve fiber layer. PFDN2 (green) was localized to RGC cytoplasm and the nerve fiver layer. To-PRO-III iodide (blue) counterstained the nuclei. Merged images are shown in A and E. In a human donor eye with no diagnosis of disease (I-L) and a donor with glaucoma (M-P), SNCG localization patterns and relative levels were identical to those found in mice. Merged images are shown in I and M. Arrows in E and M mark reduced expression of SNCG in the glaucomatous retina. $\mathrm{GCL}=$ ganglion cell layer, $\mathrm{NFL}=$ nerve fiber layer. Scale bars: $10 \mu \mathrm{m}$. 
immunoreactivity (Figure 6B, 6F, 6J, 6N), as we did not observe changes in PFDN2 levels between healthy and diseased retinae (Figure 3-8C, G, $\mathbf{K}$ and $\mathbf{O}$ ). These data demonstrate that SNCG expression is limited to RGCs, which corroborates with previously published studies in RGCs, yet PFDN2 is expressed in RGCs as well as other cells throughout the retina.

\section{Quantitative and qualitative analyses of SNCG and PFDN2 in isolated RGCs}

Using dissociated retinal cells and flow cytometry, we provide additional evidence to support our hypothesis that both SNCG and PFDN2 are co-expressed in RGCs. Each dissociated mouse retina yielded on average $5 \times 10^{6} \pm 1.2$ cells based on trypan blue exclusion. Flow cytometry analyses revealed that we consistently obtained greater than 95 $\pm 2.4 \%$ live cells (Figure 3-9). Downstream analyses were performed exclusively on live cells. To investigate the percentage of retinal cells that expressed both PFDN2 and SNCG, we used intracellular labeling. Figure 3-10 (left panel) shows that only a small percentage $(<2 \%)$ of live retinal cells are $\mathrm{SNCG}^{+}$. We expected that the percentage would be small considering SNCG is expressed solely by RGCs which comprise $\sim 0.5 \%$ of all retinal cells (148). In contrast, all live murine retinal cells were positive for PFDN2, a ubiquitously expressed chaperone protein (Figure 3-10 middle panel). Among experiments, we measured an average of $100 \pm 0 \%$ and $1.5 \pm 0.4 \%$ of total retinal cells that express PFDN2 and SNCG, respectively. Retinal cells probed simultaneously with antibodies for both proteins showed that $<2 \%$ of live retinal cells have the PFDN2 ${ }^{+} \mathrm{SNCG}^{+}$phenotype (Figure 3-10, right panel).

Intracellular labeling using anti-SNCG and anti-PFDN2 antibodies allow for robust identification of RGCs, but it renders them unviable for downstream investigations because the cells are fixed and permeabilized as they become metabolically inactive. To overcome this shortcoming, we isolated primary RGCs using FACS and cell surface antibodies. As shown in Figure 3-11, we performed a positive selection using a Thy ${ }^{+}$ (Pan-Thy1) antibody and a negative selection using a CD48 antibody to remove monocytes, macrophages and microglia. To verify that the live Pan-Thy $1^{+} \mathrm{CD} 48^{\text {neg }}$ cells were RGCs, we performed intracellular labeling on a small aliquot, as detailed above.

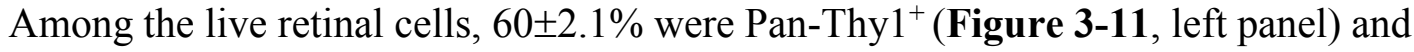
$23 \pm 3.6 \%$ of them were $\mathrm{CD} 48^{+}$(Figure 3-11, middle panel). This positive and negative selection strategy allowed us to enrich for RGC with a Live Pan-Thy $1^{+} \mathrm{CD} 48^{\text {neg }}$ phenotype. We further verified this phenotype using the SNCG intracellular marker and demonstrate that $45 \pm 3.5 \%$ of enriched cells are immunopositive for SNCG. This indicates that our enrichment strategy increases the number of RGCs 30 -fold $(45 \pm 3.5 \%$ vs $15 \pm 0.4 \%$ ) compared to the number found within the retina (Figure 3-11, right panel).

Enriched RGCs with a Live PanThy $1^{+} \mathrm{CD} 48^{\text {neg }}$ phenotype were maintained in RGC media at $4{ }^{\circ} \mathrm{C}$ overnight after sorting to allow for restoration of the plasma membrane. We then labeled cells with $1 \mu \mathrm{M}$ CFSE to determine cellular proliferation and quantified CFSE fluorescence over time to determine cell viability and proliferative status so they could be used in downstream testing (Figure 3-12). Flow cytometry 

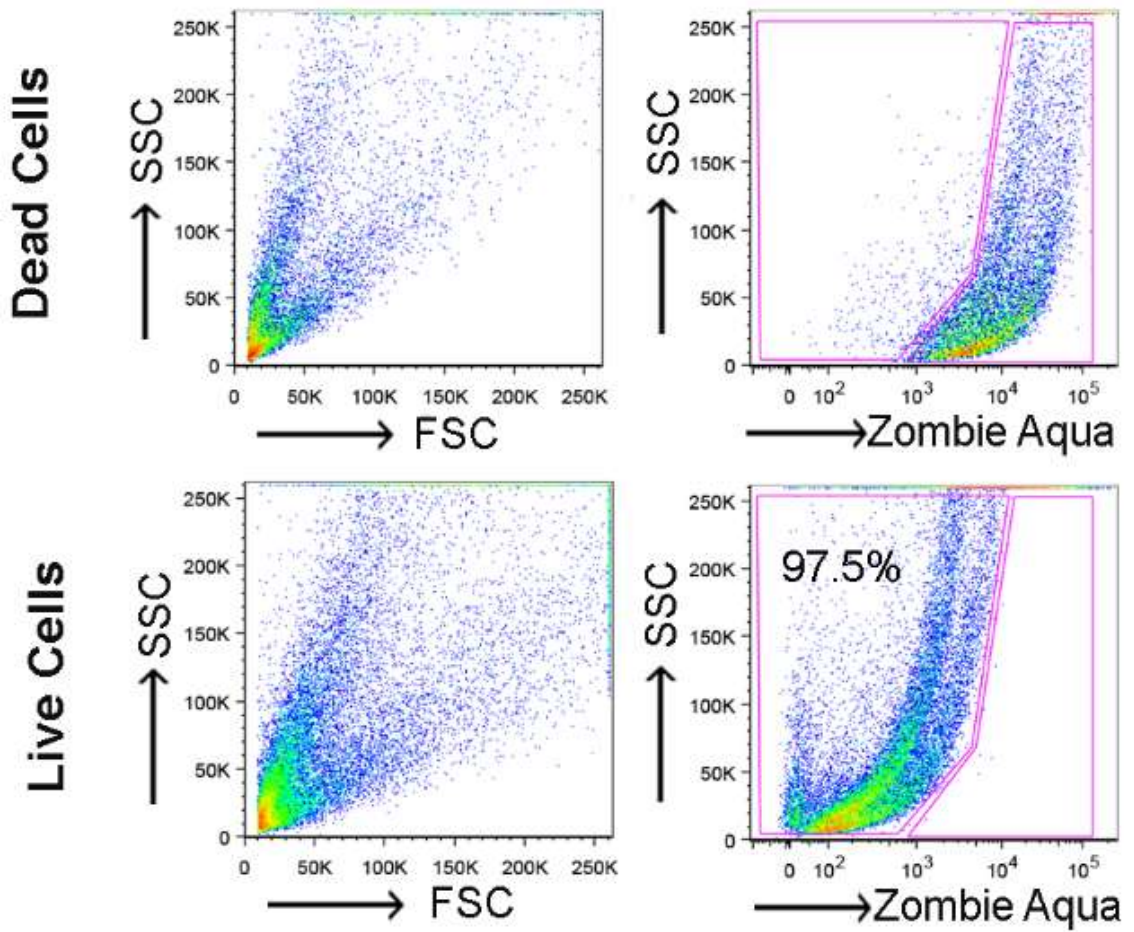

Figure 3-9. Dissociated retinal cells were labeled with Zombie Aqua ${ }^{\text {TM }}$ for live/dead cell analysis

Top Panel, representative pseudocolor plot showing Zombie Aqua ${ }^{+}$cells, which depicts dead cells. Bottom Panel, in our cell suspension approximately $97.5 \%$ of cells are alive, based on exclusion of Zombie Aqua ${ }^{\mathrm{TM}}$ dye. 

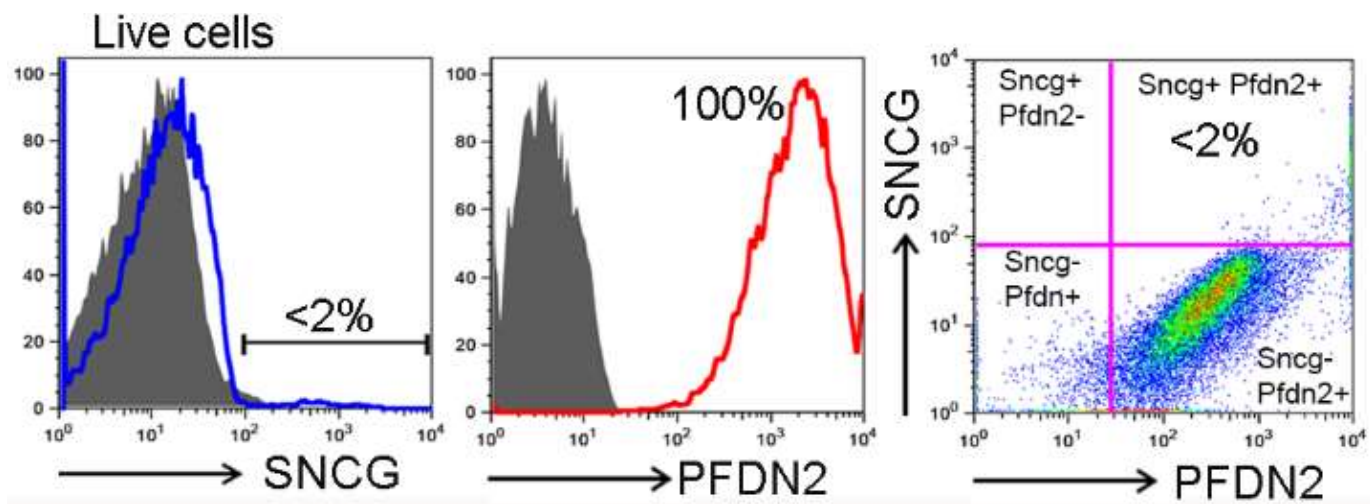

Figure 3-10. Histogram analysis showing the percentage of retinal cells labeled with anti-SNCG anti-PFDN2 antibodies

Pseudocolor plot shows $<2 \%$ of retinal cells are positive for both SNCG and PFDN2.

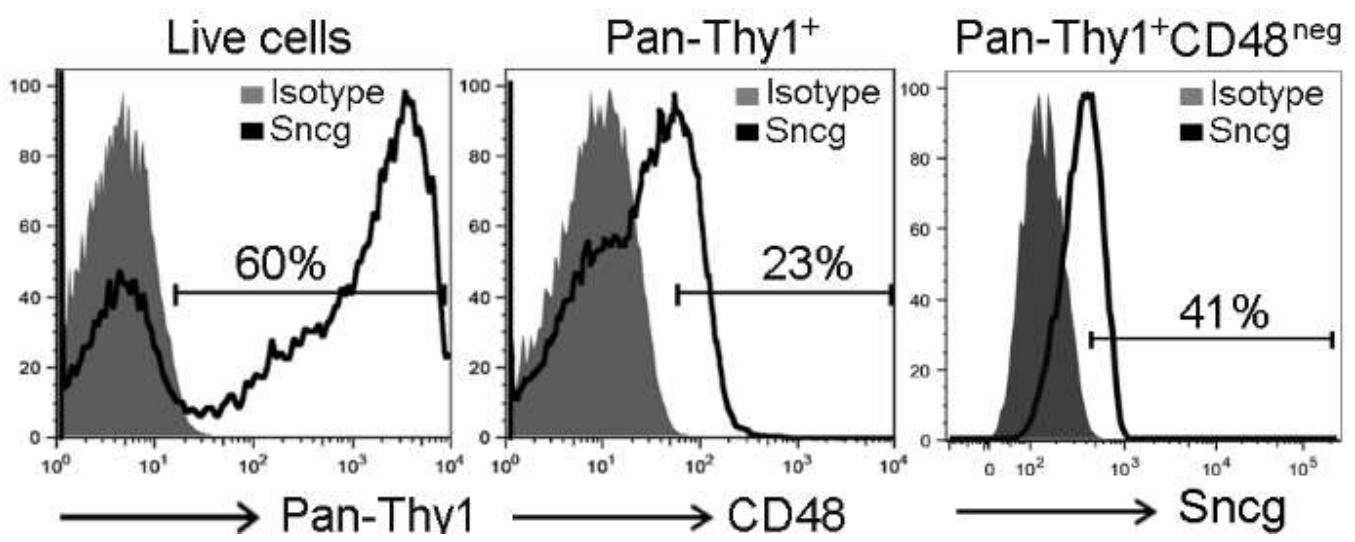

Figure 3-11. Cell enrichment strategy

Cells were positively selected based on Pan-Thy1 (Thy1.1 and Thy1.2 antibodies) staining. The Pan-Thy $1^{+}$population was negatively selected for CD48 to have a final phenotype of Live Pan-Thy $1^{+} \mathrm{CD} 48^{\text {neg }}$ cells. An aliquot of the sorted cells was evaluated for intracellular SNCG labeling, which exhibit $41 \%$ positivity. 


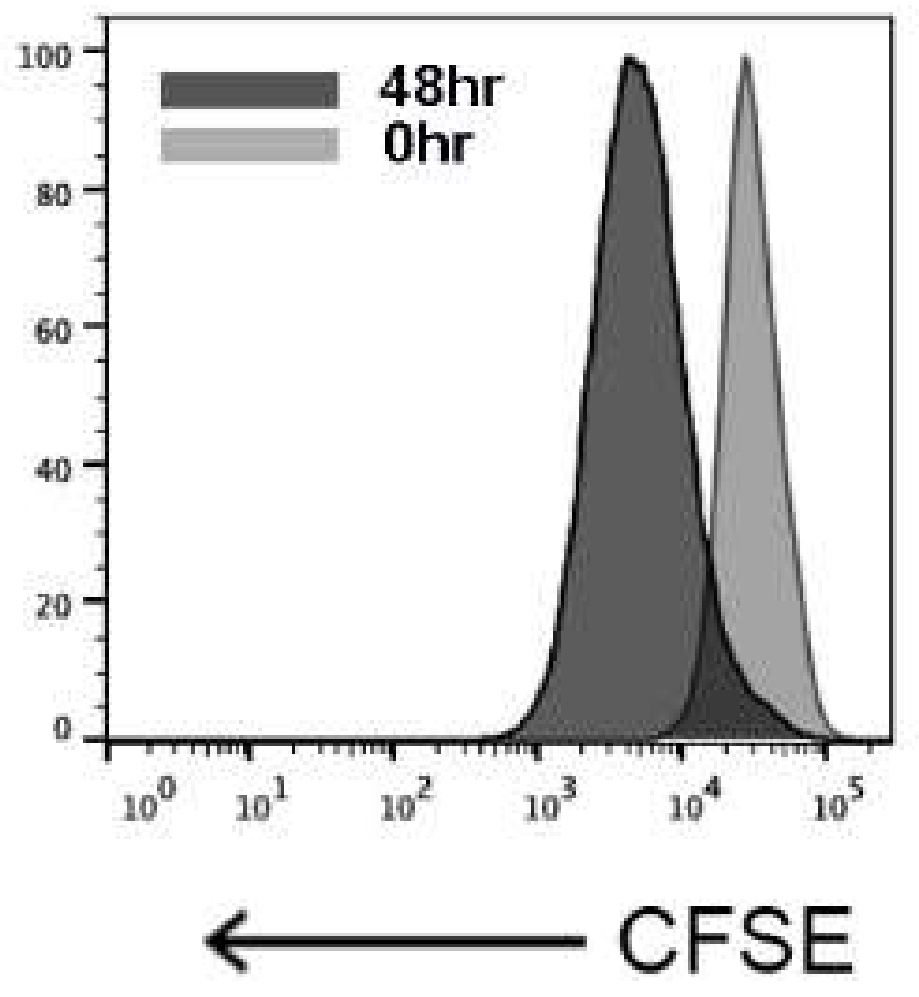

Figure 3-12. Cellular proliferation was measured by CFSE labeling

Distribution shift indicate that sorted Pan $\mathrm{Thy}^{+} \mathrm{CD} 48^{\text {neg }}$ cells undergo cell division. 
analysis revealed that RGCs were alive and underwent mitotic division (Figure 3-12), thus making them competent for mechanistic studies.

\section{siRNA mediated knockdown of $P f d n 2$ significantly reduced the expression of $S n c g$ in enriched RGCs}

To investigate whether the enriched RGCs are suitable for siRNA transfection, cell survival was measured by using Zombie Aqua ${ }^{\mathrm{TM}}$ viability dye (BioLegend, San Diego, CA) after siRNA transfection. No significant difference $(p>0.05)$ in viability was observed between the mock-transfected cells and those transfected with control siRNA or $P f d n 2$ siRNA (Figure 3-13A). To directly investigate if $P f d n 2$ modulates $S n c g$ expression in enriched RGCs, the levels of $P f d n 2$ and $S n c g$ mRNA transcripts were measured 48hours after transfecting the cells with $P f d n 2$ siRNA. Quantitative transcriptional analyses demonstrate that both $S n c g$ and $P f d n 2$ are expressed in enriched primary RGCs (Figure 3-13B). A significant knockdown of $P f d n 2$ expression $(61 \%$ reduction; $p \leq 0.001)$ was observed in the cells transfected by Pfdn2 siRNA compared to cells exposed to control siRNA. Furthermore, knockdown of $P f d n 2$ expression using siRNA resulted in a significant reduction in the expression of $S n c g$ mRNA compared to samples treated with control siRNA $(57 \%$ reduction; $p \leq 0.001)$ and samples without any siRNA treatment $(89 \%$ reduction; $p \leq 0.001)$ (Figure 3-13B), demonstrating that decreasing $P f d n 2$ expression levels caused a significant reduction in $S n c g$ expression in primary enriched murine RGCs. These results strongly suggest that $P f d n 2$ modulates Sncg in RGCs.

The transcriptional downregulation of $P f d n 2$ reduced PFDN2 and SNCG proteins coded by $P f d n 2$ and $S n c g$, respectively. Flow cytometry analyses (Figure 3-13C) after cell transfection resulted in a significant reduction in the number of PFDN2 $2^{+}$cells $(18.8 \pm 8.2 \%$ when transfected with $P f d n 2$ siRNA compared to $39.1 \pm 3.8 \%$ when transfected with Control siRNA, $p=0.049)$. Under this same experimental condition, we also found a statistically significant reduction in the percentage of enriched RGCs that were immunopositive for SNCG $(10.2 \pm 10.1 \%$ when transfected with $P f d n 2$ siRNA compared to $41.7 \pm 0.6 \%$ when transfected with Control siRNA, $p=0.047)$. Collectively, our results show that knockdown of $P f d n 2$ using siRNA results in a decrease in both the amount of Sncg transcript and the number of $\mathrm{SNCG}^{+}$cells.

\section{Network enrichment highlights the involvement of mitochondrial function}

As a next step in our investigation, we identified the top 500 genes with variation in expressions levels that were correlated with changes in Sncg or Pdfn2 expression levels. The comparison of shared correlates of $S n c g$ and $P f d n 2$ generated a list of 163 genes (Figure 3-14). This list was submitted for GO tree enrichment analysis and a directed acyclic graph was generated that grouped the transcripts according to molecular function. Ten of $32(31 \%)$ categories in the graph were statistically over-represented in the GO tree generated from the 163 shared correlates. Five out of eight (62\%) clusters 

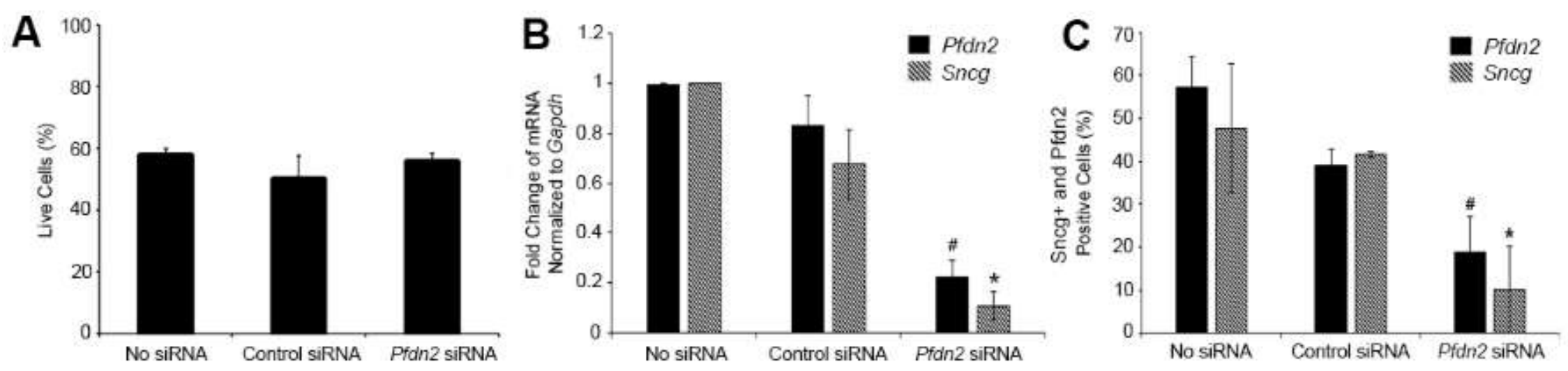

Figure 3-13. Pfdn2 knockdown inhibits Sncg expression in enriched primary RGCs

(A) Viability of retinal cells defined by Zombie Aqua ${ }^{\mathrm{TM}}$ after control siRNA and Pfdn2 siRNA transfection for 48 hours is similar to untreated cells $(p>0.05)$. (B) Transcriptional analyses for Sncg and Pfdn2 in primary mouse RGCs demonstrate a significant downregulation of $S n c g$ mRNA after $P f d n 2$ siRNA treatment $\left({ }^{*} p<0.001\right)$. There is a significant difference between the level of $P f d n 2$ expression in the control siRNA treatment group and the $P f d n 2$ siRNA treatment group (\# $p<0.001)$. There is also a significant difference_between the level of Sncg expression in the control siRNA treatment group and the Pfdn2 siRNA treatment group (* $p<0.001$ ). Results are presented as a fold change after normalizing to the level of Gapdh mRNA. (C) Protein analyses by flow cytometry show a significant reduction in the percentage of enriched RGCs that were immunopositive for SNCG and PFDN2 after $P f d n 2$ siRNA treatment. There is a significant difference between the number of cells that are immunopositive for PFDN2 (\# $p<0.05$ ) and SNCG $(* p<0.05)$ after transfection with $P f d n 2$ siRNA. Specific isotype controls were used to distinguish between positive and false positive cells. Results are shown as means \pm SEM from three independent biological replicates performed in triplicate. 


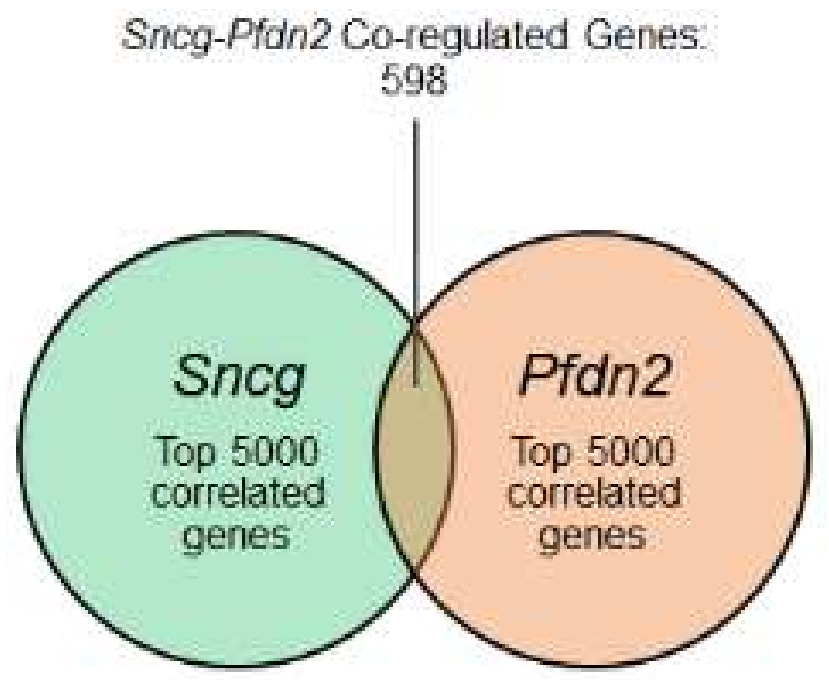

Figure 3-14. Venn diagram summarizing number of gene transcripts with expression levels that are correlated with $S n c g$ and $P f d n 2$ expression levels, along with the number of shared correlates between the two groups 
contained genes related to mitochondrial function: "Oxidoreductase activity" (11 genes); "Hydrogen ion transmembrane transporter activity" (4 genes); and "Structural constituent of ribosome" (4 genes); "NADH dehydrogenase activity" (2 genes); and "Cytochrome-c oxidase activity" (2 genes) (Figure 3-15; Appendix B, Table B-3 to B-7). The gene products encoded by these transcripts are present in mitochondria, and are involved in maintaining the potential difference across the inner mitochondrial membrane maintaining normal physiological function (Figure 3-16) (149-169).

\section{Discussion}

In this investigation, we have combined cutting edge methodologies of systems genetics, bidirectional studies using multiple species, meta-analyses, immunohistochemistry, FACS sorting and gene knockdown studies to identify and validate the identity of a genetic modulator of $S n c g$, a gene that has been previously implicated in RGC death in glaucoma. Our investigation identified $P d f n 2$ as an upstream regulator of Sncg in RGCs. In healthy retinae, the protein levels of both genes are significantly correlated in a positive manner. In addition, they are co-localized in the cytoplasm of RGCs and in the nerve fiber layer. Targeted downregulation of $P d f n 2$ in an enriched population of murine RGCs resulted in both the suppression of Sncg mRNA and also in the number of enriched RGCs that express SNCG. We have further demonstrated using two mouse glaucoma models-BXD66 and DBA/2J, and the retina from a human donor glaucoma patient, that the relationship between the two genes is modified in the diseased state. Specifically, in glaucoma, the relationship between the genes appears to have changed to an inverse relationship with reduced Sncg levels as the disease progressed. SNCG protein levels also dropped to nearly negligible levels in RGCs from subjects with advanced disease, while PDFN2 levels have remained steady.

Previously, linkage analyses and clustering methods have been used to identify candidate genes, such as_complement component 1a (Clqa), that were associated with glaucoma in BXD strains (170). In our investigation, we went a step farther and utilized partial correlation analysis to solidify the relationship between $S n c g$ and $P f d n 2$ in the mouse retina. The statistically significant partial correlation between $S n c g$ and $P f d n 2$ reflects gene-gene interactions, as well as a regulatory relationship between the genes. The partial correlation approach has successfully been developed and applied to transcription data from yeast (171), Arabidopsis thaliana (172), HeLa cells (173), and breast cancer tumors (174). To our knowledge, it has not previously been used to study the interactions between two genes in the mouse retina.

Sncg has been suggested to be a marker for glaucoma due to the association between RGC degeneration and the loss of Sncg mRNA and protein expression in both human glaucoma patients and animal models of glaucoma $(139,142,143)$. In neuroretinal cells, SNCG plays an essential regulatory role in resistance to stress, and neuroprotection (175). Another report demonstrated that a reduction in SNCG protein levels initiates an apoptotic death cascade due to the dephosphorylation of BCL2-binding protein (176). In addition, the loss of Sncg correlates with increased RGC death as shown 


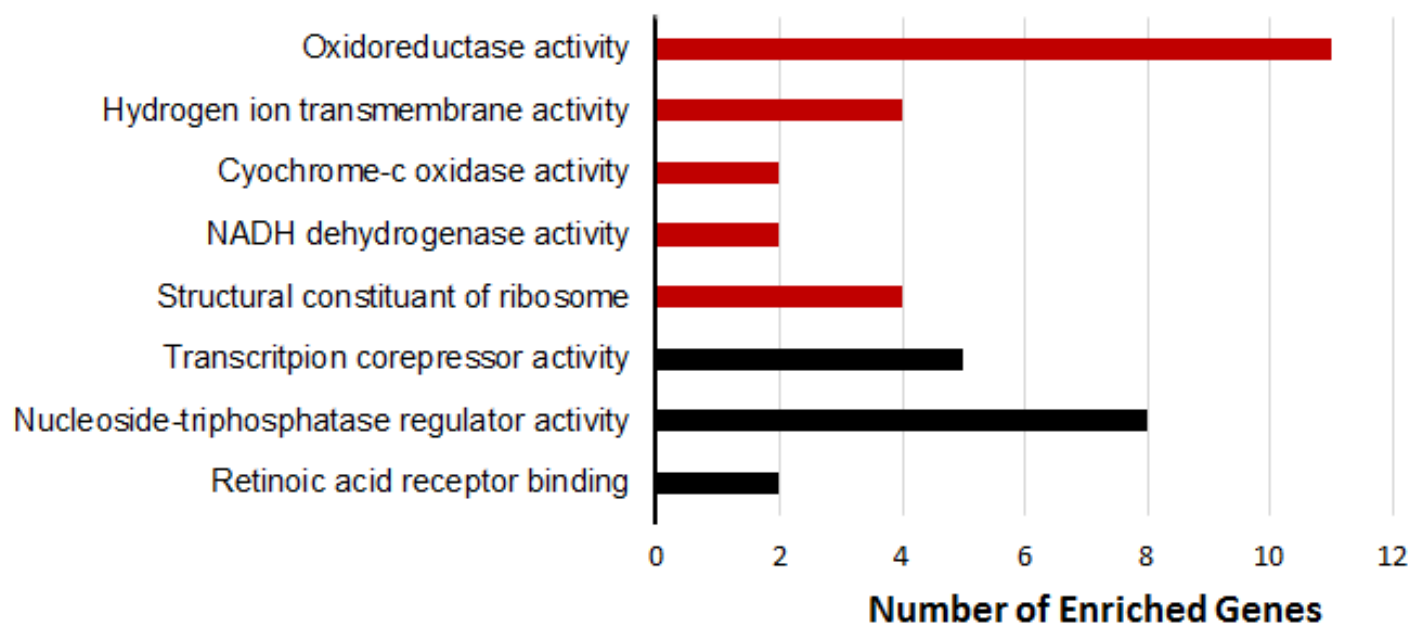

Figure 3-15. Gene ontology analysis of the shared correlates of $\operatorname{Sncg}$ and $P f d n 2$ are presented

Categories that are statistically over-represented are presented (adjusted $p=5.9 \times 10^{-2}$ for all groups). Categories indicated with a red bar indicate mitochondria-associated genes 


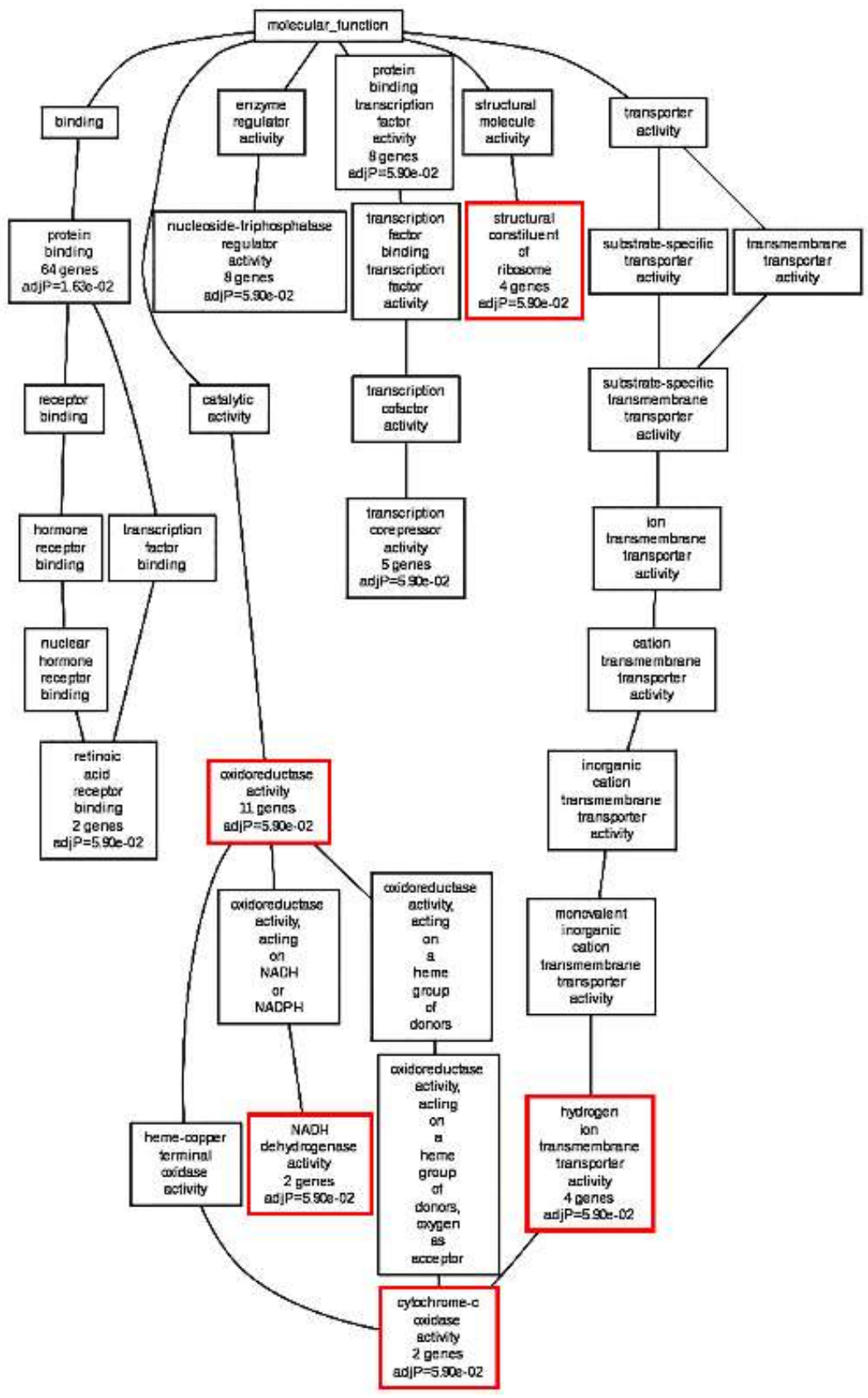

Figure 3-16. Gene ontology analysis of the shared correlates of Sncg and Pfdn2

Categories that are statistically over-represented are presented in (adjusted $p=5.9 \times 10^{-2}$ for all groups). Categories indicated with a red boxes indicate mitochondria-associated genes. 
in DBA/2J mice (143). Apart from its neuroprotective role,_SNCG acts as a molecular chaperone (177) by interacting with myocilin, a glycoprotein that when mutated is associated with glaucoma (178). Collectively, the literature suggests that SNCG plays a critical role in RGC health, yet the regulation of this gene had not yet been previously investigated.

PFDN2 is a ubiquitously expressed protein in the retina as a heterohexameric cochaperone that in eukaryotes and it is also a component of the prefoldin complex (179). Of direct relevance to our investigation, prefoldins 1-6 are neuroprotective and they specifically prevent the aggregation of misfolded proteins, such as $\alpha-$ synuclein (180, 181). When mutated, $P f d n 2$ is likely involved in the formation of toxic $\beta$-amyloid oligomers that are a common pathology of multiple neurodegenerative diseases (182) In addition, Lee et al., have reported that $P f d n 5$ is crucial for normal sensory and neuronal development (183). Mutations in other family members, such as $P f d n 3$, cause structural disorganization of neuronal microtubules and microfilaments (184). Several lines of evidence suggest that the main cytoskeletal proteins, including microtubule-associated proteins and neurofilaments, are not normally transported along the ocular axon stump of degenerated RGCs, which leads to apoptosis (185). Strong immunolabeling of SNCG and PFDN2 in the nerve fiber layer of both mouse and human retinae in our study suggests a possible role of these genes in microtubule regulation or trafficking in axons of RGCs.

$P f d n 2$ is located in the QTL rich region (Qrrl) on Chr 1, which is a genomic region of unusually high gene density and contains major regulatory QTLs for various behavioral, metabolic, physiologic and immunological processes $(179,186,187)$ including diverse epileptic traits (188). The distal portion of Qrrl is strongly implicated in modulating RNA metabolism, translation, tRNA aminoacylation, and transportation of transcripts into neuronal dendrites (186). Qrrl also modulates behavioral alterations in respect to activity and anxiety $(189,190)$. Physiologically relevant genes such as formin2 (Fmn2), regulator of G-protein signaling $7\left(\operatorname{Rgs}_{\mathrm{S}} \mathrm{)}\right.$ ) and a cluster of tRNAs are modulated by the Qrrl distal region. However, none of these genes had significant partial correlation values with $S n c g$ and therefore they were excluded from the list of candidate upstream modulators of this Sncg.

In glaucomatous DBA/2J mice, we identified an inverse relationship between Sncg and Pfdn2. Specifically, mice that have the highest degree of degeneration have the lowest $S n c g$ level, yet the highest level of $P d f n 2$ expression. Using both qualitative and quantitative methods, we demonstrate that in compared to healthy individuals with no disease, SNCG is reduced in the retina of glaucoma patients. These findings are shared by both humans with a diagnosis of glaucoma and a novel BXD model of glaucoma. It is plausible that in glaucoma that RGCs upregulate Pdfn2 in an attempt to increase $S n c g$ expression. Its known that glaucoma induces stress to the RGCs, which leads to damage to proteins in the cells. Stress induced synthesis of chaperones is impaired in in diseases such as glaucoma. It is plausible that reduction in $S n c g$ expression in aging DBA/2J is an effect of age-related changes associated with glaucoma, to protect the cells from damage $P f d n 2$ expression upregulates to compensate for the loss of chaperone function in RGC. 
Due to the complex cellular heterogeneity of the retina, it is relatively difficult to reliably obtain a pure RGC population from retina. In particular, the comparative analysis of molecular regulation or profiles is hampered by the lack of an adequate methodology for the selective purification of RGCs from retinae. I have addressed this caveat in Chapter 4 of this dissertation. A representative histogram shows that using our enrichment protocol $41 \%$ of the sorted live enriched RGCs were SNCG-positive. Moreover, our CFSE results indicate that these cells are viable and are capable of dividing for at least $48 \mathrm{hrs}$ under specific cell culture conditions. We have also been successful in keeping enriched RGCs alive for upto 6 days. The cell culture conditions that we used does not include serum. Presence of serum in culture has the potential to alter cellular responses dramatically. Serum-free media has proven to be critical both in addressing how RGCs behave independently of other signals and in identifying the molecular signals that mediate specific neuronal-glial interactions in the CNS (191-195). Addition of trophic factors such as BDNF, CNTF, and insulin which are usually made by cells in contact with RGCs promote RGC health and survival. The present strategy allows for the enrichment population of murine RGCs that retain the integrity of their proteins, DNA and RNA for subsequent downstream biochemical and molecular analyses.

Using our enriched primary RGCs, we used a siRNA-based approach to knockdown $P f d n 2$ gene expression and measure the effect upon $S n c g$ expression. Our data show that targeted siRNA successfully downregulated $P f d n 2$ by $\sim 61 \% \mathrm{p} \leq 0.001$ at 48 hour post transfection. Comparative gene expression analyses revealed an equally effective downregulation of Sncg in enriched RGCs treated with Pfdn2- targeted siRNA. Our data demonstrate that, $P f d n 2$ is upstream of $S n c g$ and it directly modulates $S n c g$ expression in enriched mouse RGCs. The majority of the biological process categories represented in the GO enrichment analysis from $S n c g$ and $P f d n 2$ shared correlates are associated with mitochondrial structure and function. RGCs are responsible for transmitting visual information from the retina to the lateral geniculate nucleus. Due to this large anatomical distance, RGCs must transport metabolites and organelles via axonal transport, a process with a high-energy demand. Mitochondria play a key role in the life and death of neurons. Any dysfunction of axonal transport or mitochondrial function can have severe consequences on RGC function and viability $(196,197)$. Additional studies show that mitochondria are a direct target of $\alpha$-synuclein in Parkinson's disease (198), which lends support for our findings. Several lines of evidence suggest that transport of mitochondria within a cell occurs by interactions with a variety of cytoskeletal proteins which contributing to maintenance cellular physiology (199, 200).

In summary, our results show that genetic mapping and network modeling can be combined with multiparametric flow cytometry and gene silencing to identify and verify the genetic regulation of a retinal gene. Ours is the first investigation in which the relationship between $S n c g$ and $P f d n 2$ has been established in both the intact retina and in enriched primary murine RGCs. The discovery of this relationship may help in guiding studies that explore the disease mechanisms associated with altered protein transport and folding in RGCs. In glaucoma the identification and confirmation of these two proteins in 
RGC health and disease holds great promise for developing molecular targets to slow RGC damage, which in turn will preserve vision. 


\section{CHAPTER 4. ISOLATION AND VALIDATION OF PRIMARY MURINE RGCS FROM HEALTHY AND DAMAGED RETINAE*}

\section{Introduction}

Glaucoma is a complex multifactorial disease characterized by the progressive dysfunction and loss of RGCs and associated visual field deficits. It remains a leading cause of vision loss affecting 70 million people worldwide (201). In spite of notable technical advances, the cellular mechanisms underlying their loss remain unclear. This is in part due to the lack of standardized, reliable protocols to isolate large numbers of highly enriched RGCs and/ or a RGC line for in vitro mechanistic studies $(202,203)$. Identifying the genetic basis or cellular mechanisms causing RGC degeneration would be the first step towards development of efficacious therapies to slow or reverse RGC damage, in turn preserving vision. The lack of a validated RGC population represents a large unmet need for the vision research community at large.

The isolation and enrichment of primary murine RGCs is essential for investigating RGC responses to specific therapies in vitro. A number of challenges have prevented progress towards the use of a homogeneous primary murine RGC population. First, a major challenge lies on the scarce number of RGCs that can be isolated from murine retinae $(148,204,205)$. Second, current signature markers for the identification of RGCs are intracellular markers (206-208), impeding the isolation of viable, metabolically active cells downstream for in vitro studies. Third, current protocols are lengthy and have not been standardized for the isolation of primary murine RGCs from dissociated retinae. Barres et al. (209) adapted the immunopanning technique into a two-step process to purify RGCs. The process includes depletion of macrophages and endothelial cells, followed by positive selection of cells responding to anti-Thy1. Recently, Hong (210) optimized a similar process that included positive selection of Thy $1^{+}$cells using magnetic beads followed by cell sorting. Both approaches require lengthy isolations and their yields are inconsistent. A commercial kit is available for isolating RGCs from retinae (211), however, it has two major limitations: the kit is for exclusive use in rats, yet mice are the primary animal model used in vision research; and the specificity of this kit for RGCs is debatable, as amacrine cells could also be isolated with this method. In recent years the use of Dynabeads or flow cytometry in conjunction with monoclonal antibodies (212) or lectins (213) has provided powerful tools to improve the purity of isolated cells.

Flow cytometry, also known as FACS, is a powerful method that analyzes cell suspensions and provides quantitative and qualitative data with a high level of sensitivity. FACS cellular discrimination is based on physical properties such as surface area and the

\footnotetext{
* Reprinted with permission from 2007-2016 Frontiers Media SA. Chintalapudi SR, Djenderedjian L, Stiemke AB, Steinle JJ, Jablonski MM, Morales-Tirado VM. Isolation and Molecular Profiling of Primary Mouse Retinal Ganglion Cells: Comparison of Phenotypes from Healthy and Glaucomatous Retinas. Front Aging Neurosci. 2016 May 18;8:93. doi: 10.3389/fnagi.2016.00093. eCollection 2016. PubMed PMID: 27242509 ; PubMed Central PMCID: PMC4870266.
} 
internal complexity or granularity of the cells (214). Multi-dimensional analysis, based upon the expression of proteins on the cell surface as well as intracellularly, can be performed by the combination of antibodies tagged with fluorochromes. Current FACSbased cell sorting techniques allow for the separation of up to four different cell populations based on multivariate properties. Sorted cells can be collected and are viable for downstream analyses.

In the present study, we developed a novel flow cytometry-based protocol to generate a homogeneous RGC population from murine retinae. We employed a highly stringent sort strategy coupled with qualitative PCR (qPCR) and intracellular staining with RGC-signature markers to verify the purity and homogeneity of the enriched population. Our isolation technique provides a powerful tool for vision research to assist in the understanding of the molecular pathways and key players in preservation of RGC function and health to develop novel therapies for vision loss (Figure 4-1).

\section{Methods}

\section{Dissociation of murine retinae}

Two hundred C57BL/6J mice between 5-7 weeks of age, 22 BXD66 mice ages 5 weeks (young) and $>12$ months (old) were used in this study. All procedures were approved by the Institutional Animal Care and Use Committee (IACUC) review board at the University of Tennessee Health Science Center (UTHSC) and followed the Association for Research in Vision and Ophthalmology (ARVO) Statements for the Use of Animals in Ophthalmic and Vision Research, in addition to the guidelines for laboratory animal experiments (Institute of Laboratory Animal Resources, Public Health Service Policy on Humane Care and Use of Laboratory Animals). Mice were sacrificed by cervical dislocation followed by enucleation, as previously described in (215). Retinae were dissociated using enzymatic digestion. The resultant cell suspension was filtered using a Falcon $70 \mu$ m nylon strainer (BD Biosciences, San Jose, CA) followed by centrifugation at 1500 RPM x 5 minutes at RT. Cells were resuspended in PBS/1\% FBS (Lonza, Walkersville, MD) and kept on ice until ready for use.

\section{Flow cytometry analyses}

\section{Cell surface labeling}

Cell viability was evaluated at the time of retinae cell dissociation. To ensure we obtain live cells after sorting, we labeled the cells with Zombie Aqua ${ }^{\mathrm{TM}}$ (BioLegend, San Diego, CA) a permeant dye to discriminate between live (negative for dye) and dead (positive for dye) cells. Live cells were treated with $1.0 \mu \mathrm{g}$ of anti-CD16/CD32 per $1.0 \times 10^{6}$ cells in $100 \mu \mathrm{L}$ to block FcyRII/III (clone 93; BioLegend), which minimizes nonspecific binding of the primary antibodies and in turn inhibits endocytosis, phagocytosis 


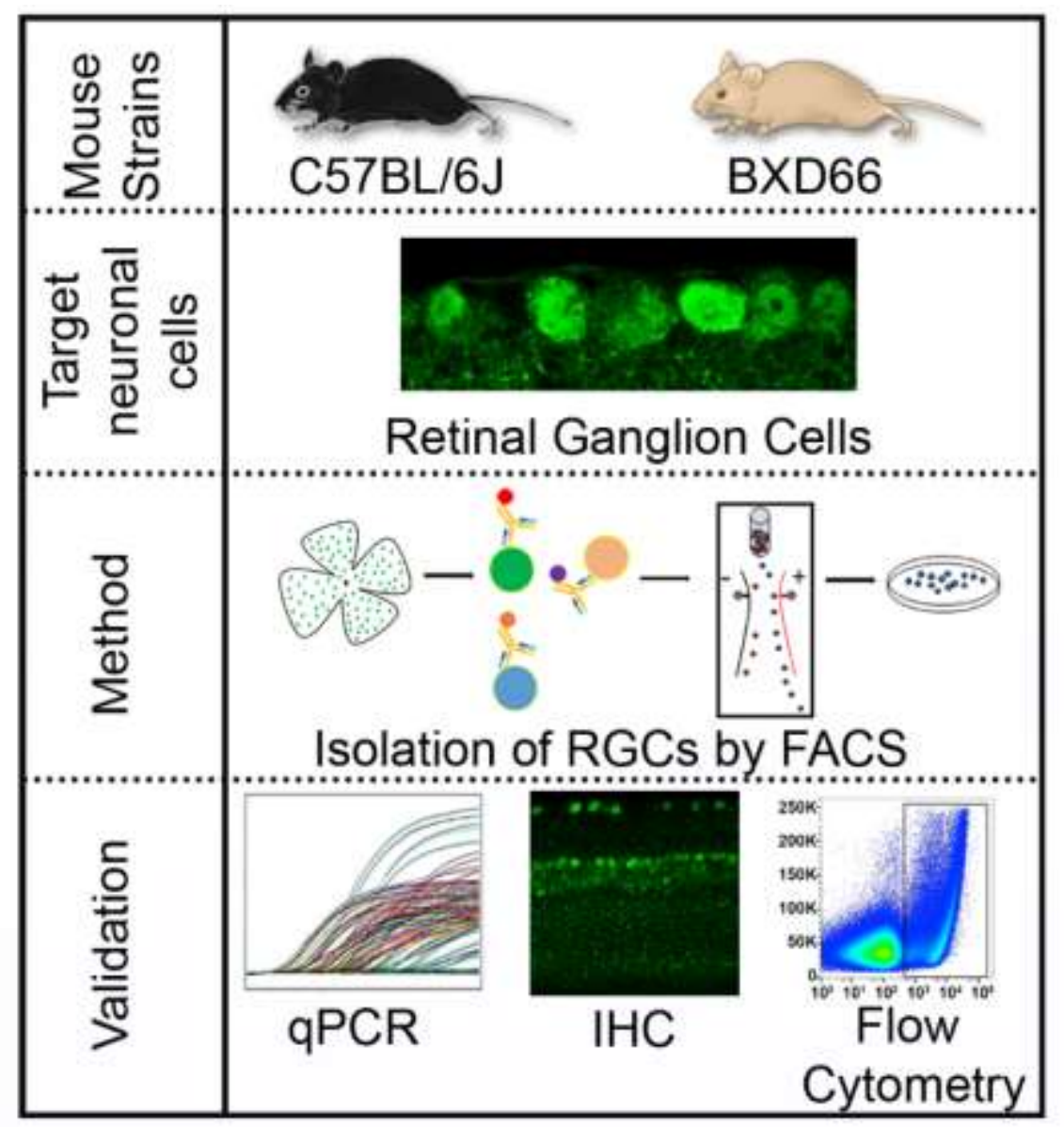

Figure 4-1. Illustrated summary

Loss of functional RGCs is an element of retinal degeneration that is poorly understood. This is in part due to the lack of a reliable and validated protocol for the isolation of primary RGCs. Here we optimize a feasible, reproducible, standardized flow cytometrybased protocol for the isolation and enrichment of homogeneous RGC with the Thy $1.2^{\text {hi }} \mathrm{CD} 48^{\text {neg }} \mathrm{CD} 15^{\text {neg }} \mathrm{CD} 57^{\text {neg }}$ surface phenotype. A three-step validation process was performed by: (1) genomic profiling of 25-genes associated with retinal cells; (2) intracellular labeling of homogeneous sorted cells for the intracellular RGC-markers SNCG, BRN3A, TUJ1, and RBPMS; and (3) by applying the methodology on RGC from a mouse model with elevated IOP and optic nerve damage. Use of primary RGC cultures will allow for future careful assessment of important cell specific pathways in RGC to provide mechanistic insights into the declining of visual acuity in aged populations and those suffering from retinal neurodegenerative diseases. 
and antigen presentation due to Fc $\gamma \mathrm{R}$ activation. The following primary antibodies were used to detect surface antigens by incubating the cells on ice for 30 minutes: anti-CD90.1 PerCP-Cy5.5 (Thy1.1, clone OX-7, BioLegend, exhibits no cross-reactivity with CD90.2); anti-CD90.2 Alexa Fluor-700 (Thy1.2, clone 30-H12, BioLegend, exhibits no cross-reactivity with CD90.1); anti-CD48 PE-Cy7 (clone HM48-1, BioLegend, labels monocytes and microglia); anti-CD15 PE (clone MC-480, BioLegend, labels amacrine cells); and anti-CD57 (clone VC1.1, Sigma Aldrich, St. Louis, MO, also labels amacrine cells). Because the anti-CD57 antibody was unconjugated, a Brilliant violet 421-tagged secondary antibody (Life Technologies, Carlsbad, CA) was used to allow for sorting.

\section{Sorting strategy}

Cells were enriched by fluorescent activated cell sorting (FACS) using a BD Biosciences FACSAria ${ }^{\mathrm{TM}}$ Cell Sorter equipped with 4-lasers (BD Biosciences, San Jose, CA). In this investigation, we used the $488 \mathrm{~nm}$ blue, the $630 \mathrm{~nm}$ red and the $405 \mathrm{~nm}$ violet diode lasers. Dissociated cells were maintained at $4^{\circ} \mathrm{C}$ using a temperature controlled sample injection and collection chamber. At the time of cell sorting, we used unlabeled murine retinal cells as controls and individual samples were labeled with antibodies specific for different retinal cell surface markers. We used AbC TM Total Antibody Compensation Bead Kit (Life Technologies) to prepare single color controls using the manufacturer's protocol. These are highly sensitive and efficient antibody capture beads with a broad multispecies reactivity. To determine the efficiency of the sort, we took a small sample of the eluted sorted sample followed by immediate FACS analysis.

\section{Intracellular labeling}

Dissociated cells labeled as detailed above followed by $1 \mathrm{hr}$ fixation at $4{ }^{\circ} \mathrm{C}$ using the BD Cytofix/ Cytoperm ${ }^{\mathrm{TM}}$ fixation/permeabilization solution (BD Biosciences) as in $(216,217)$. Cells were incubated for $1 \mathrm{hr}$ at $4^{\circ} \mathrm{C}$ in the following antibodies that were diluted in BD Perm/Wash buffer: anti-RBPMS (rabbit polyclonal IgG; Santa Cruz Biotechnology, Santa Cruz, CA; 1:100 dilution); anti-SNCG (rabbit polyclonal IgG; GeneTex, 1:100 dilution), BRN3A (goat ployclonal IgG; Santa Cruz Biotechnology; 1:100 dilution); and anti-TUJ1 (mouse monoclonal IgG2a; Covance; 1:100 dilution). The appropriate Alexa Fluor 488 tagged secondary antibodies (1:200 dilution; Invitrogen) were used to allow for data acquisition and analysis. Cells were kept in PBS/1\%FBS on ice until the time of analysis. Data acquisition was performed on a BD LSRII Flow Cytometer (BD Biosciences) and analyses were performed using FlowJo vX10.0.6 (Tree Star, Inc., Ashland, OR). To confirm results we performed additional data acquisition using the Miltenyi Biotec MACSQuant ${ }^{\circledR} 10$ Analyzer (Myltenyi Biotec, San Diego, CA).

\section{Flow cytometry and confocal microscopy dual analysis}

Labeled retinal cells were acquired based on area and aspect ratio, gating out cell debris and cell aggregates from the analysis using the Amnis ${ }^{\circledR}$ FlowSight $^{\circledR}$ Imager (EMD $^{(2)}$ Millipore, Amnis Division, Seattle, WA). Flow cytometry features include a violet (405 $\mathrm{nm}, 100 \mathrm{~mW})$, blue $(488 \mathrm{~nm}, 60 \mathrm{~mW})$, red $(642 \mathrm{~nm}, 100 \mathrm{~mW})$, and SSC (785 nm, $8 \mathrm{~mW})$ 
lasers. Data were analyzed in IDEAS software after compensation of single color control samples using a compensation matrix. Confocal images were taken at 20x.

\section{Gene analyses}

\section{RNA isolation and $c D N A$ synthesis and pre-amplification of cDNA template}

RNA from 5.0x10 $0^{5}$ sorted Live Thy $1.2^{+} \mathrm{CD} 48^{\text {neg }} \mathrm{CD} 15^{\text {neg }} \mathrm{CD} 57^{\text {neg }}$ cells was extracted following the Qiagen ${ }^{\circledR}$ miRNeasy Mini Kit (Qiagen, Valencia, CA) manufacturer's specifications. RNA concentration was assessed using a Nanodrop Spectophotometer (Nanodrop, Wilmington, DE). cDNA was synthesized from RNA using the SuperScript ${ }^{\circledR}$ VILO ${ }^{\mathrm{TM}}$ cDNA Synthesis Kit (Life Technologies). Following the manufacturer's instructions, we used 100ng of RNA for each reaction. Briefly, the preamplification reaction mixture was prepared using cDNA, TaqMan ${ }^{\circledR}$ PreAmp Master Mix and the pooled primer mix listed in Appendix C, Table C-1. Pre-amplification: the enzyme activation step was carried out at $95^{\circ} \mathrm{C}$ for $10 \mathrm{mins}$, followed by 14 cycles at $95^{\circ} \mathrm{C}$ for $15 \mathrm{sec}$ followed by $60^{\circ} \mathrm{C}$ for 4 mins. Subsequently, the pre-amplified cDNA was diluted 1:10 in TE buffer and was kept at $-20^{\circ} \mathrm{C}$ until ready for use. The pre-amplification of cDNA step was crucial to increase the sensitivity of detection for downstream quantification using qPCR. As part of our stringent validation and confirmation techniques, we used a series of gene targets that are specific for different retinal cell populations, including a housekeeping gene (Hprt). The primers that were used for the pre-amplification step are listed in the Appendix C, Table C-1.

\section{qPCR analysis amplification efficiency test}

We determined the primer efficiency and amplification efficiency by the absolute quantification method using a Roche LightCycler ${ }^{\circledR} 480$ Instrument and Version 1.5.0 Software (Roche, Indianapolis, IN). Pre-amplified cDNA was serially diluted to a range of concentration $(1,1: 10,1: 100,1: 1000)$. This comparison ensured that the preamplification process amplified genes with a wide variation of abundance. The comparative threshold $\left(\mathrm{C}_{\mathrm{T}}\right)$ values were plotted against the log concentration $\mathrm{qPCR}$ product and the slope was calculated. The closer the slope is to -3.33 , the closer the amplification efficiency is to the $100 \%$ ideal, which indicates that there is a doubling of product per cycle. In addition, we performed linear regression analysis to show the correlation between gene expression measurements from our samples for the primers we validated, and a standard curve. In this study, we used $C_{T}$ values ranging from 15-30 in our assays. Three biological replicates were evaluated and the $\mathrm{C}_{\mathrm{T}}$ values were normalized to the endogenous gene (Hprt) control and compared to the $\mathrm{C}_{\mathrm{T}}$ values obtained from the pre-amplifed cDNA from murine, non-sorted retinal cells.

\section{qPCR reaction}

For qPCR reaction we prepared a final volume of $10 \mu \mathrm{L}$ PCR reaction mixture using TaqMan ${ }^{\circledR}$ Universal Master Mix, diluted pre-amplified cDNA, primers and 
Nuclease Free water. Plates were analyzed on a Roche LightCycler ${ }^{\circledR} 480$. Instrument conditions included first a hold step of $50^{\circ} \mathrm{C}$ for 2 mins followed by $95^{\circ} \mathrm{C}$ for $10 \mathrm{mins}$. Next, we performed 40 cycles of $95^{\circ} \mathrm{C}$ for $15 \mathrm{sec}$ followed by $60^{\circ}$ for $1 \mathrm{~min}$. All measurements were made in replicates of 4 . Relative quantification was performed using $\mathrm{C}_{\mathrm{T}}$ after determining the values of $\mathrm{C}_{\mathrm{T}}$ for the reference gene (housekeeping) and the target genes in each sample. The relative fold change $\left(R_{\mathrm{q}}\right)$ was calculated using the following formula: $R_{\mathrm{q}}=2^{-\Delta \mathrm{C}} \mathrm{T}$, where $\Delta \mathrm{C}_{\mathrm{T}}=\left(\mathrm{C}_{\mathrm{T}}\right.$ target gene $-\mathrm{C}_{\mathrm{T}}$ reference gene $)$. Data are presented as mean $\pm \mathrm{SEM}$. Differences between two means were assessed with ANOVA and Tukey's post-hoc test (PRISM, Graph Pad, La Jolla, CA). Differences were considered significant at $p<0.05$.

\section{Immunohistochemistry}

Murine retinal sections embedded in low melting point agarose were prepared following our published methods (144). Briefly, tissue sections were blocked with 10\% goat serum and permeabilized with $2.5 \%$ Triton X-100. The following primary antibodies were used per manufacturers conditions: anti-RNA-Binding Protein With Multiple Splicing (RBPMS; rabbit polyclonal IgG, Santa Cruz Biotechnology, 1:100 dilution); anti- $\gamma$-synuclein (SNCG, rabbit polyclonal IgG, GeneTex, 1:100 dilution); anti-BrainSpecific Homeobox/POU Domain Protein 3A (BRN3A, goat ployclonal IgG, Santa Cruz Biotechnology, 1:10 dilution); anti-Neuronal Class III $\beta$-Tubulin (TUJ1, mouse monoclonal IgG2a, Covance, Princeton, NJ; 1:100 dilution); anti-HNK-1/N-CAM (CD57, Clone VC1.1, mouse monoclonal IgM, 1:10 Dilution); and CD15 (Clone:MC480, mouse monoclonal IgM, Biolegend, 1:25 dilution). The appropriate Alexa Fluortagged secondary antibodies (Invitrogen, Waltham, MA; 1:200 dilution) and TO-PRO3 iodide (Invitrogen; 1:4000 dilution) were used to indicate the location of the antigens of interest and nuclei, respectively. Sections were viewed and images were obtained using a Nikon C1 (Nikon, NY) confocal microscope within the Imaging Core Facility in the Hamilton Eye Institute. All microscope settings, including laser levels and gain, were held constant to allow for relative comparisons of signal intensity within and between experiments.

\section{Immunofluorescence}

Live Thy $1.2^{+} \mathrm{CD} 48^{\text {neg }} \mathrm{CD} 15^{\text {neg }} \mathrm{CD} 57^{\text {neg }}$ sorted cells $(50,000$ cells) were washed once with Hanks Balanced Salt Solution (HBSS) in followed by centrifugation at 1200 RPM x 5 mins at RT. Cells were labeled with SYTO ${ }^{\circledR} 59(100 \mathrm{nM})$ for 10 mins in a HBSS at $37^{\circ} \mathrm{C}$ followed by multiple washes with HBSS for 5 mins each. Cells were plated on a glass bottom microwell dish (35mm petri dish, 14mm microwell; MatTek Corporation, Ashland, MA) and sealed with coverslip. Analysis performed using a Nikon C1 (Nikon, NY) confocal microscope within the Imaging Core Facility in the Hamilton Eye Institute. 


\section{Optic nerve processing, imaging and counting}

Eyes along with optic nerves were enucleated from mice immediately after euthanasia. Optic nerves were cut close to the globe and were fixed in $0.8 \%$ paraformaldehyde and $1.22 \%$ glutaraldehyde in $0.08 \mathrm{M}$ phosphate buffer. They were subsequently rinsed in buffer and post-fixed in $1 \%$ osmium tetroxide. After dehydration, the specimens were embedded in Epon 812 plastic. Sections $(0.8 \mu \mathrm{m}$ thick) were cut on an ultramicrotome (Reichert-Jung Ultracut E Ultramicrotome), stained with pphenylenediamine (PPD) for $30 \mathrm{~min}$. Digital images were taken using 10x and $4 \mathrm{x}$ objectives using a MicroFire digital camera (Optronics ${ }^{\circledR}$, Goleta, CA) mounted onto a Nikon Eclipse E800 light microscope (Nikon). Multiple contiguous photomicrographs were taken at 60x magnification to provide a continuous representation across the optic nerve. A scale of optic nerve damage similar to that used by Clark and colleagues was used to assign a numeric value to the appearance of the nerve (David Cantu-Crouch, IokHou Pang, Mitchell D. McCartney, Abbot F. Clark; unpublished protocol). The numeric value is based upon the presence/absence of darkly stained axoplasm and presence of gliotic scars.

\section{IOP measurements}

IOP was measured using the induction-impact tonometer (Tonolab tonometer, Colonial Medical Supply, Franconia, NH) for rodents according to the manufacturer's recommended procedures. When measuring IOP, the tonometer was fixed in a vertical position to a support stand by means of clamps. The mouse was gently restrained by hand on an adjustable stand, and the eye was oriented in such a way that a distance of 1-4 mm was maintained between from the tip the probe to the cornea of the eye. Six consecutive IOP readings were averaged. IOP readings obtained with Tonolab have been shown to be accurate and reproducible in various mouse strains, including DBA/2J.

\section{Results}

\section{The Thy $1^{+}$CD $48^{\text {neg }}$ surface phenotype is not sufficient to identify murine RGCs}

Murine retinal cells express two distinct isoforms of Thy1-Thy1.1 and Thy1.2 (218-220). We compared the binding of Thy1.1 and Thy1.2 to dissociated murine retinal cells and show the majority of murine retinal cells exhibit immunoreactivity against Thy1.2 but not Thy1.1 (Figure 4-2, Thy $1.2^{+}$at $53.9 \%$ versus Thy $1.1^{+}$at $1.7 \%$ ). Current RGC isolation techniques use selection based on Thy1-positivity and CD48-negativity by antibody-capture $(209,210)$ or magnetic cell isolation $(211,213)$. Our data demonstrate that a small percent of Thy $1.2^{+}$cells were also immunopositive for CD48 (11.2\%) and that inclusion of CD48 as a negative selection marker did not significantly decrease the number of Thy $1.2^{+}$cells (Figure 4-3, left panel). To determine if the Thy $1.2^{+} \mathrm{CD} 48^{\text {neg }}$ cells were RGCs, we probed the cells for the expression of the RGC signature marker $\gamma$ - 

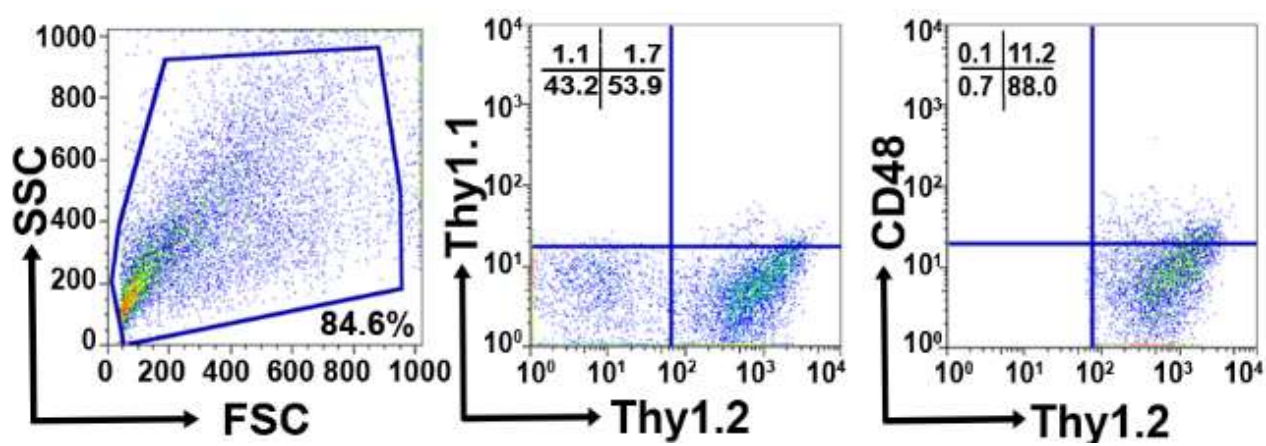

Figure 4-2. Thy $1^{+} \mathrm{CD} 48^{\text {neg }}$ surface phenotype is not sufficient to identify murine RGCs

Left panel: Pseudocolor plots were gated on live nucleated cells based on FSC and SSC profiles. Based upon these characteristics, $84.6 \%$ of the cells were live after retinal dissociation. Middle panel: A negligible number of live retinal cells were Thy $1.1^{+}$ $(1.1 \%)$, whereas $53.9 \%$ were Thy $1.2^{+}$, demonstrating that in mouse Thy 1.2 is the preferred surface marker. Right panel: The majority of the Thy $1.2^{+}$cells $(88 \%)$ were CD $48^{\text {neg }}$.
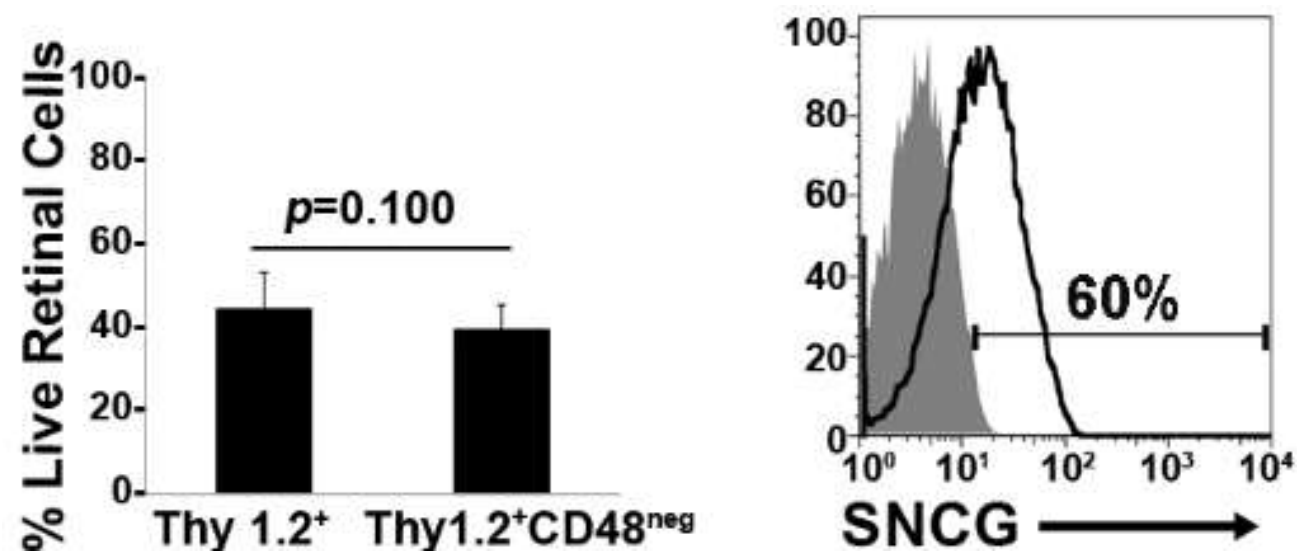

Figure 4-3. Lack of specificity of current RGC isolation methods

There was no significant difference in the percent of live Thy $1.2^{+}$and Live Thy $1.2^{+} \mathrm{CD} 48^{\text {neg }}$ cells demonstrating that the addition of CD48 as a negative selection marker is insufficient to further enrich for RGCs. Only $60 \%$ of LiveThy $1.2^{+} \mathrm{CD} 48^{\text {neg }}$ are $\mathrm{SNCG}^{+}$indicating that many contaminating cell types remain in the LiveThy $1.2^{+} \mathrm{CD} 48^{\text {neg }}$ cell population. Gray histogram represents control and black line represents experimental sample. 
synuclein (SNCG). Consistently, we found that a large proportion of Thy $1.2^{+} \mathrm{CD} 48^{\text {neg }}$ cells were not $\mathrm{SNCG}^{+}$. Figure 4-3 right panel, illustrates that $60 \%$ of Live Thy $1.2^{+} \mathrm{CD} 48^{\text {neg }}$ cells were positive for SNCG, suggesting that there is $\sim 40 \%$ contamination by non-SNCG ${ }^{+}$cell types. To address which non-RGC cell types are present within the Thy $1.2^{+} \mathrm{CD} 48^{\text {neg }} \mathrm{SNCG}^{\text {neg }}$ phenotype, we performed qPCR analysis on the sorted cells. Using the absolute quantification method, we determined the optimal concentration of pre-amplified samples that would be suitable for our validation step and calculated the efficiency of amplification (Figure 4-4). Our qPCR method is presented in Figure 4-4. Results shown in Figure 4-5 reveal the degree of heterogeneity of the enriched RGCs using only Thy1.2 and CD48 as surface markers. Specifically, Thy $1.2^{+} \mathrm{CD} 48^{\text {neg }}$ cells expressed genes associated with multiple retinal cells, including amacrine, Müller, bipolar, horizontal, photoreceptors and retinal pigment epithelial cells. Table 4-1 lists the specific genes associated with the various cell types. Our data demonstrates that selection based solely on Thyl and CD48 expression is insufficient to isolate highly enriched RGCs.

\section{Confirmation of additional surface markers to be used as negative selectors and intracellular RGC markers}

We used IHC to determine the retinal localization patterns of other surface markers that were expressed by contaminating cells. CD15 has been described as a marker of retinal interneurons including amacrine and bipolar cells (221), while CD57 has been shown to label glial cells and photoreceptors (222). Our data demonstrate that CD15 (Figure 4-6A) is localized in the interface between the inner nuclear layer (INL) and inner plexiform layer (IPL) and in the proximal INL (arrows), where amacrine cells are located. Occasional $\mathrm{CD} 15^{+}$cells were observed in the ganglion cell layer (GCL), which are most likely displaced amacrine cells (arrows). CD57 immunoreactivity (Figure 4-6B) was abundant in the outer plexiform layer (OPL) and in a radial pattern through the INL. Punctate staining was also present in the GCL, which are likely astrocytes or displaced amacrine cells. Collectively, these results show that these surface markers-CD15 and CD57—can be used as negative selectors to remove non-RGCs from the Thy $1.2^{+} \mathrm{CD} 48^{\text {neg }}$ cell population.

To verify that the commonly accepted RGC intracellular markers are specific for RGCs $(175,208,212,223)$, we labeled murine retinal sections with antibodies against SNCG, BRN3A [Brain-specific homeobox/POU domain protein 3A (Pouf4l)], TUJ1 [neuron-specific class III beta tubulin (Tubb3)] and RBPMS [RNA binding protein with multiple splicing (Rbpms)]. SNCG showed abundant expression in the GCL (Figure 4-7A). Abundant BRN3A (Figures 4-7B) labeling was observed in the GCL. However, multiple cells in the INL that border the IPL are also immunopositive for BRN3A. Based upon their location, they are likely amacrine cells. TUJ1 (Figure 4-7C) was very abundant in the GCL and in radial labeling patterns throughout the retina that extend up to the ONL. Lastly, RBPMS was highly expressed by cells in the GCL (Figure 4-7D) and a small subpopulation of cells in the INL (arrow). Collectively, these data demonstrate that SNCG-, BRN3A-, TUJ1- and RBPMS-positive cells are present in the 

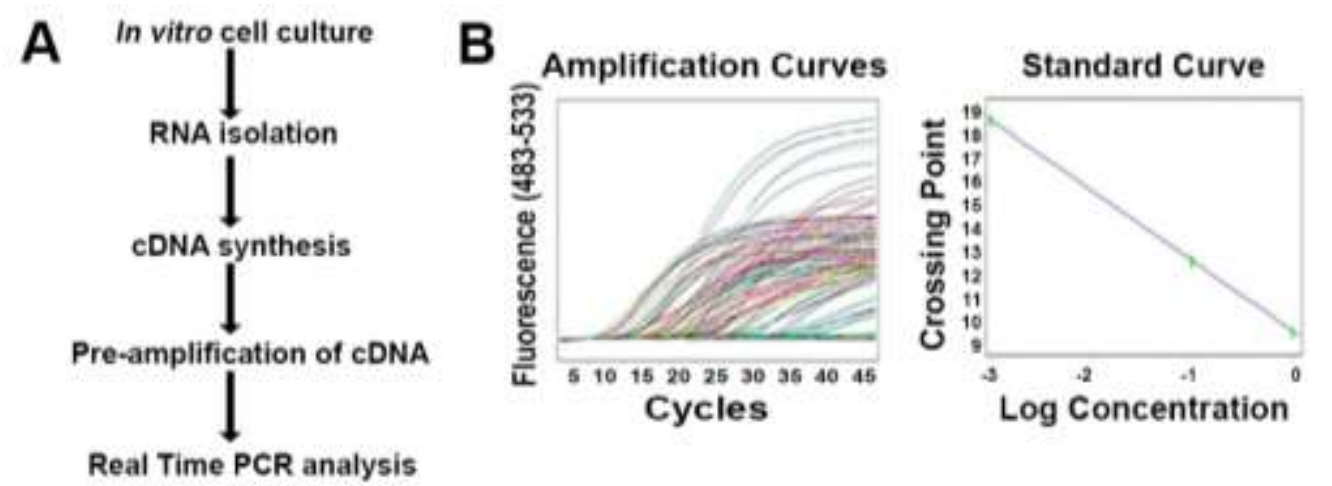

Figure 4-4. Validation of our improved protocol for the enrichment of RGCs

(A) Schematic representation of our validation analyses of Live Thy $1.2^{+} \mathrm{CD} 48^{\text {neg }} \mathrm{CD} 15^{\text {neg }} \mathrm{CD} 57^{\text {neg }}$ sorted cells. (B) Amplification and standard curves to evaluate primer efficiency. Left panel: amplification curves of the ten-fold dilution series for all the primers. Right panel: Standard curves depicting $\mathrm{C}_{\mathrm{T}}$ plotted against the $\log$ of the starting quantity of template for each dilution. The efficiency of the all PCR reactions was between $90-100 \%(-3.6 \geq$ slope $\geq-3.3)$. All samples were prepared and analyzed in triplicate. 


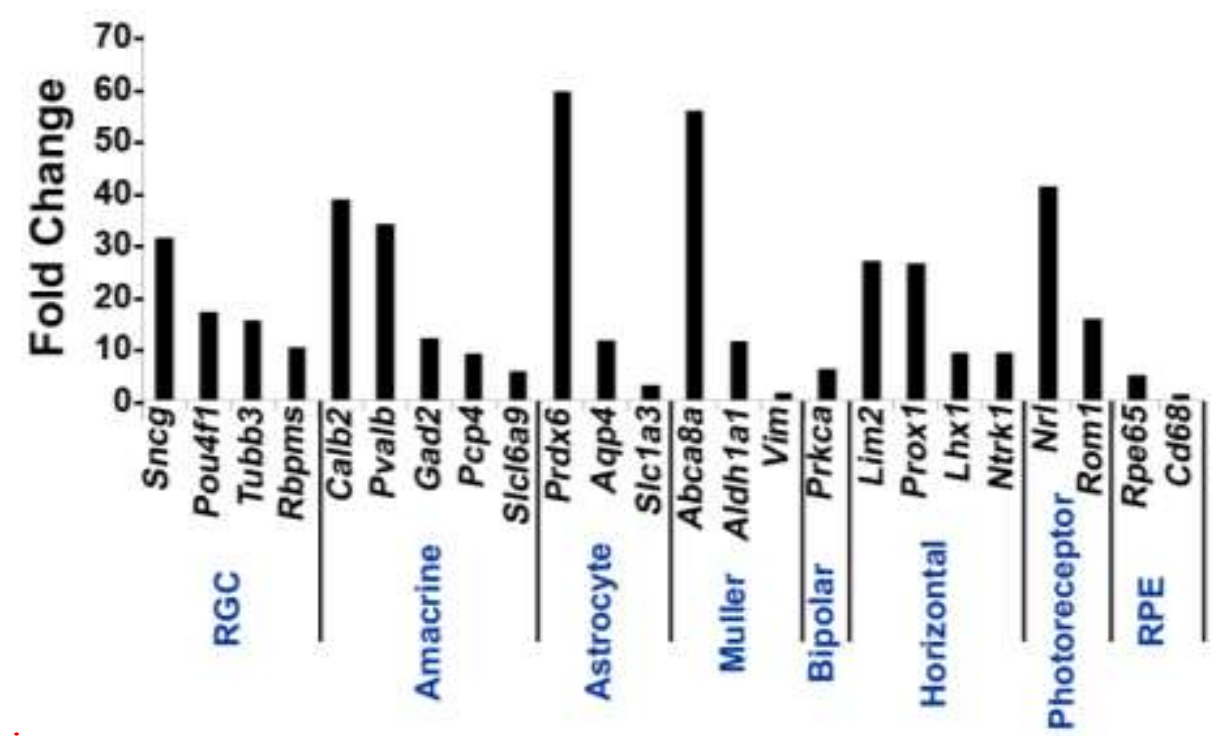

Figure 4-5. Identification of Thy $1.2^{+} \mathrm{CD}^{\mathrm{n}} \mathrm{8}^{\text {neg }}$ cells using qualitative real time PCR analysis

The expression levels of a panel of 25 genes expressed by various retinal cell types - amacrine, Müller, bipolar, horizontal, photoreceptor and retinal pigment epithelial cells - in Thy $1.2^{+} \mathrm{CD} 48^{\text {neg }}$ cells were normalized to the levels present in unsorted total retinal cells. The Thy $1.2^{+} \mathrm{CD} 48^{\text {neg }}$ population showed contamination with other retinal cell types. Target gene expression levels are presented as $\log _{2}$ fold change based on the $\Delta \mathrm{C}_{\mathrm{T}}$ calculation using Hprt as a housekeeping gene and water as negative control.

Mean \pm SEM. 
Table 4-1. Genes used in gene expression analyses as part of the validation of our RGC enrichment protocol

\begin{tabular}{ll}
\hline Retinal Cell Type & Genes Expressed by Retinal Cell Type \\
\hline Retinal Ganglion Cell & Pou4fl; Rbpms; Sncg; Tubb3; Chrma6; Rbfox3; \\
& Nef-H \\
& Gad2; Fut4; Calb2; Pvalb; Slc6a9; Pcp4; Vip; \\
Amacrine & Thyl \\
& Aqp4; Prdx6; Gfap; Slc1a3; Pax2 \\
Astrocytes & ApoE; Abca8a; Vim; Aldh1al \\
Müller & Pkca; Pcp4; Rcvrn; Slcla2 \\
Bipolar & Rcvrn; Proxl; Ntrk1; Lhxl, Lim2, Calb2 \\
Horizontal & Nrl; Rom-1; Crx; Pxph2; Arr3 \\
Photoreceptors (Cone and Rod) & Cd68; Rpe65 \\
Retinal Pigment Epithelial Cells & Hprt \\
Housekeeping Gene &
\end{tabular}




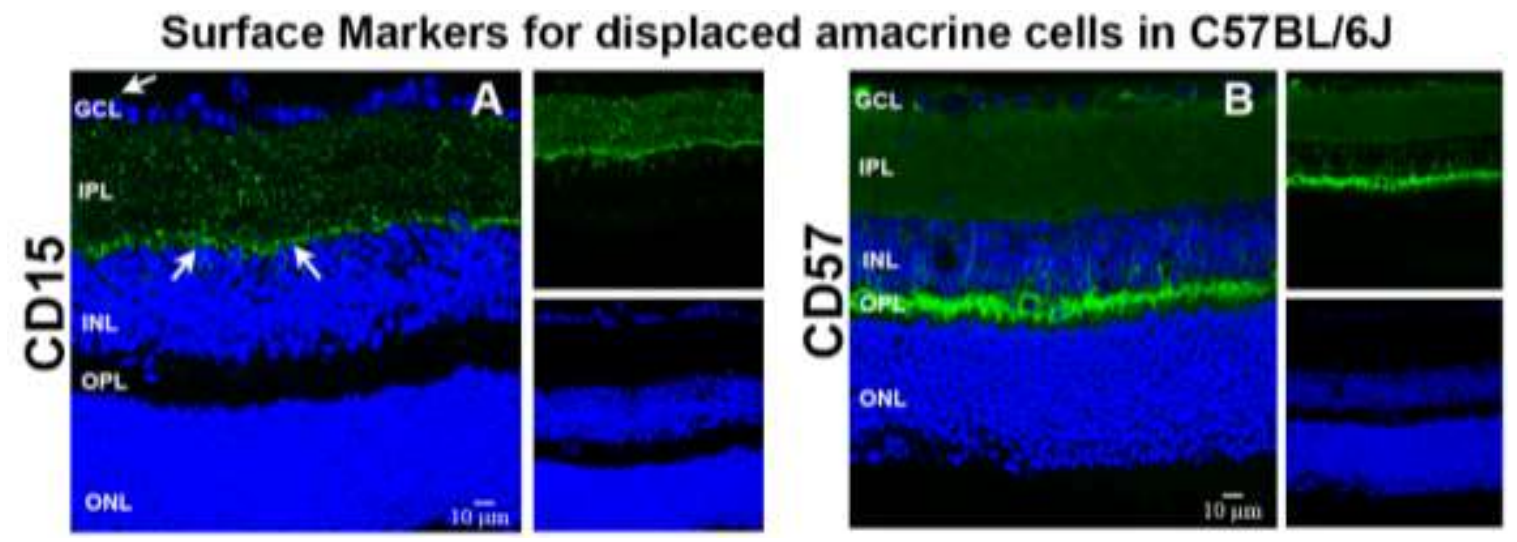

Figure 4-6. Immunohistochemical localization of surface antigens used in our RGC sorting protocol

Cellular localization of the surface proteins CD15 and CD57 in retinae from C57BL/6J mice. Sections from C57BL/6J mouse retinae were labeled with antibodies against (A) CD15 and (B) CD57. TO-PRO-III iodide staining labeled nuclei of all retinal cells (blue). Abbreviations: $\mathrm{GCL}=$ ganglion cell layer; $\mathrm{IPL}=$ inner plexiform layer; $\mathrm{INL}=$ inner nuclear layer; $\mathrm{OPL}=$ outer plexiform layer; $\mathrm{ONL}=$ outer nuclear layer. Scale bar: $10 \mu \mathrm{m}$. 


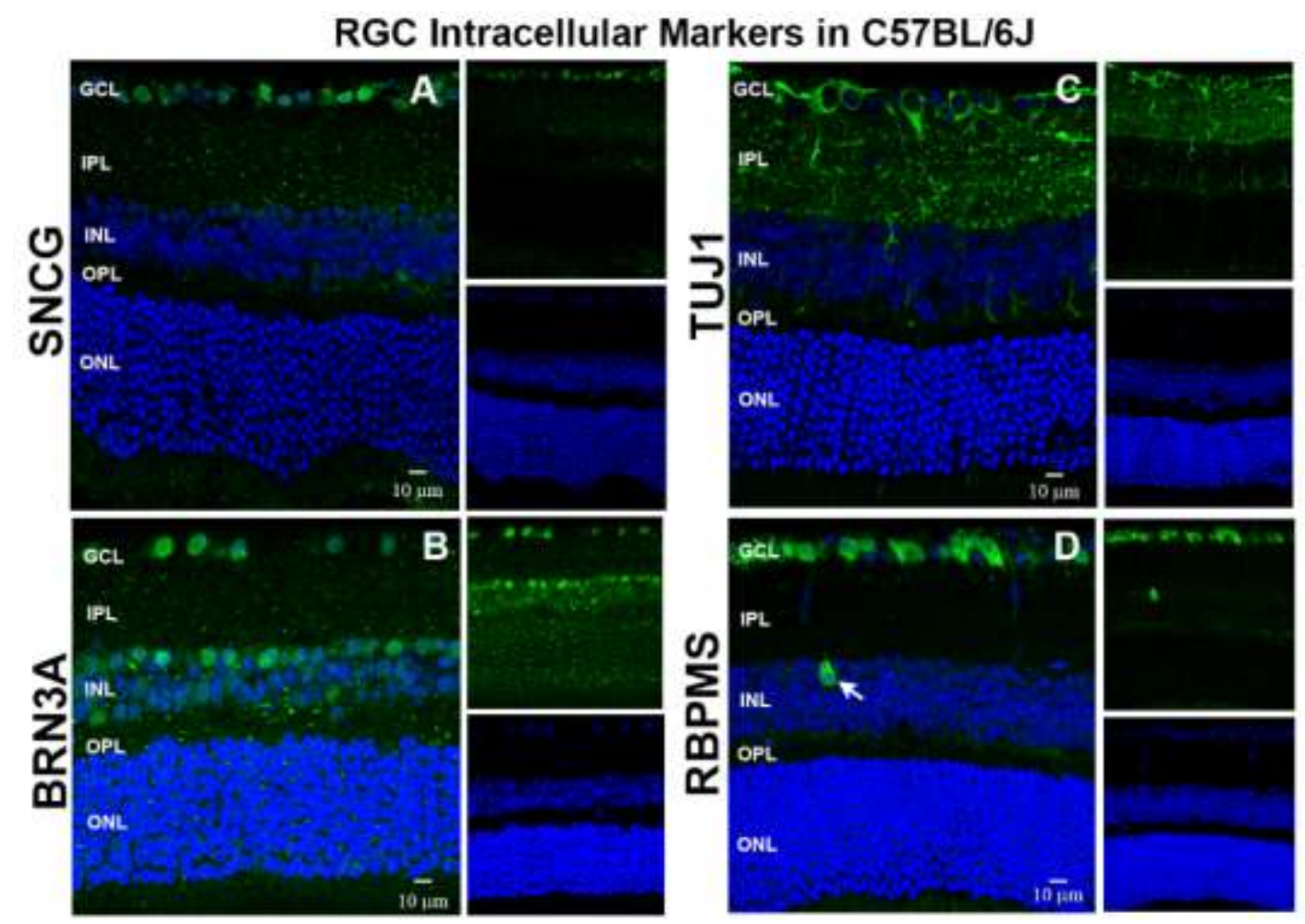

Figure 4-7. Immunohistochemical localization of intracellular RGC markers used as part of the validation process of our sorting protocol

Cellular localization of intracellular proteins SNCG, BRN3A, TUJ1 and RBPMS in retinae from $\mathrm{C} 57 \mathrm{BL} / 6 \mathrm{~J}$ mice. Sections from $\mathrm{C} 57 \mathrm{BL} / 6 \mathrm{~J}$ mouse retinae were labeled with antibodies against (C) SNCG, (D) BRN3A, (E) TUJ1, and (F) RBPMS. TO-PRO-III iodide staining labeled nuclei of all retinal cells (blue). Abbreviations: $G C L=$ ganglion cell layer; $\mathrm{IPL}=$ inner plexiform layer; $\mathrm{INL}=$ inner nuclear layer; $\mathrm{OPL}=$ outer plexiform layer; $\mathrm{ONL}=$ outer nuclear layer. Scale bar: $10 \mu \mathrm{m}$. 
GCL and can be used to validate the identity of the cells that we isolate using our array of cell surface markers. Even though BRN3A and RBPMS also label a small subset of (likely) amacrine cells, we are confident that requiring all enriched RGCs to express all four RGC markers will yield a nearly pure RGC population.

\section{Live Thy $1.2^{+} C 48^{\text {neg }}$ CD15 ${ }^{\text {neg }}$ CD57 $7^{\text {neg }}$ RGC express all signature intracellular markers SNGC, BRN3A, TUJ1 and RBPMS}

Our expanded cell sorting strategy is presented in Figure 4-8. Because Thy $1.2^{+} \mathrm{CD} 48^{\text {neg }}$ cells expressed many markers associated with retinal cells other than RGCs, we added additional surface markers - CD15 and CD57-to our sorting strategy to remove these contaminating cells. Our methodology included the negative selection of these cell surface markers to enrich for naïve RGCs that could be used in downstream analyses. Collectively, we were targeting the removal of monocytes, as well as glial, amacrine and photoreceptor cells. Prior to cell surface labeling, we added purified mouse anti-CD16/32 antibody to block Fc $\gamma$ RII/III, thus reducing false positive immunoreactivity (224) $(224,225)$. Our flow cytometry-based cell sorting validation studies included examination of pre- (Figure 4-9) and post-sorted cells (Figure 4-10) to confirm that the post-sorted cells that were isolated using cell surface markers expressed all four RGC intracellular markers: SNCG, BRN3A, and TUJ1, and RBPMS. Consistently, we observed $99-100 \%$ positivity for all of the intracellular markers in the post-sorted cells, demonstrating the isolated cells were very highly enriched, if not pure RGCs. We ensured consistency of our results across multiple systems using two other cytometer systems, the MACSQuant ${ }^{\circledR}$ Analyzer 10 and the FlowSight ${ }^{\circledR}$. Our novel method was standardized as we obtained reproducible results (Figure 4-11, Figure 4-12).

Using confocal microscopy, we investigated the morphological appearance of the sorted cells. Live Thy $1.2^{+} \mathrm{CD} 48^{\text {neg }} \mathrm{CD} 15^{\text {neg }} \mathrm{CD} 57^{\text {neg }}$ cells were stained with SYTO ${ }^{\circledR} 59$ dye showing nuclear integrity. Cells showed bright blue fluorescence and strong intracellular staining (Figure 4-13). This result confirms LiveThy $1.2^{+} \mathrm{CD} 48^{\text {neg }} \mathrm{CD} 15^{\text {neg }} \mathrm{CD} 57^{\text {neg }}$ sorted cells show the morphology associated with RGC.

\section{Validation of the enriched RGC population by qPCR analyses}

Qualitative PCR analyses (Figure 4-14) shows that the highly enriched RGC population had a many fold increase in the expression of all four RGC intracellular markers: Sncg, Pouf4l, Tubb3 and Rbpms. As expected, genes found in other retinal cells were expressed at significantly lower levels than in unsorted retinal cells. Moreover, our improved sorting methodology removed the contaminating retinal cell types that were present in the Thy $1.2^{+} \mathrm{CD} 48^{\text {neg }}$ cells (compare Figure 4-5 and Figure 4-14). These mRNA analyses further validated our RGC enrichment strategy. 


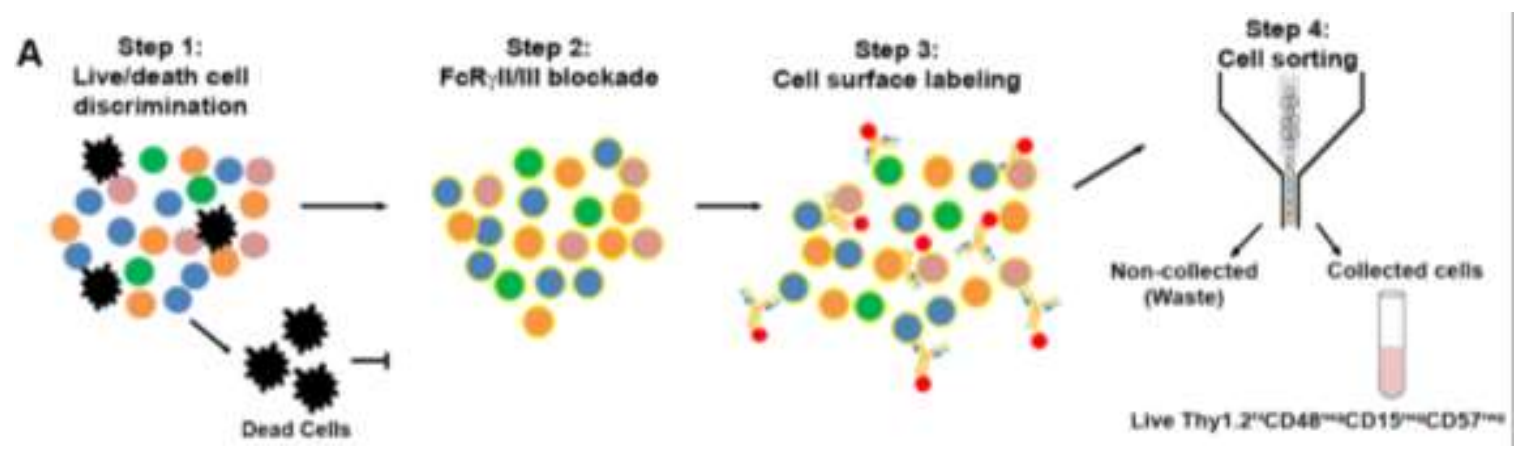

Figure 4-8. Optimized FACS-based cell sorting strategy

Schematic representation of RGC isolation by flow cytometry using multiple surface markers. Step 1: Cells were labeled with Zombie Aqua ${ }^{\mathrm{TM}}$ for live/dead cell analysis followed by step 2: blocking of Fc $\gamma$ RII/III (mouse anti-CD16/32) to minimize nonspecific labeling. Step 3: Cell surface labeling was performed using the following antibody cocktail: anti-mouse anti-CD90.2 Alexa Fluor 700; anti-CD48 PE-Cy7; antiCD15 PE and anti-CD57 Brilliant Violet 421 to yield LiveThy $1.2^{+} \mathrm{CD} 48^{\text {neg }} \mathrm{CD} 15^{\text {neg }} \mathrm{CD} 57^{\text {neg }}$ cells. Single labeled fluorochrome-beads were used as controls. 

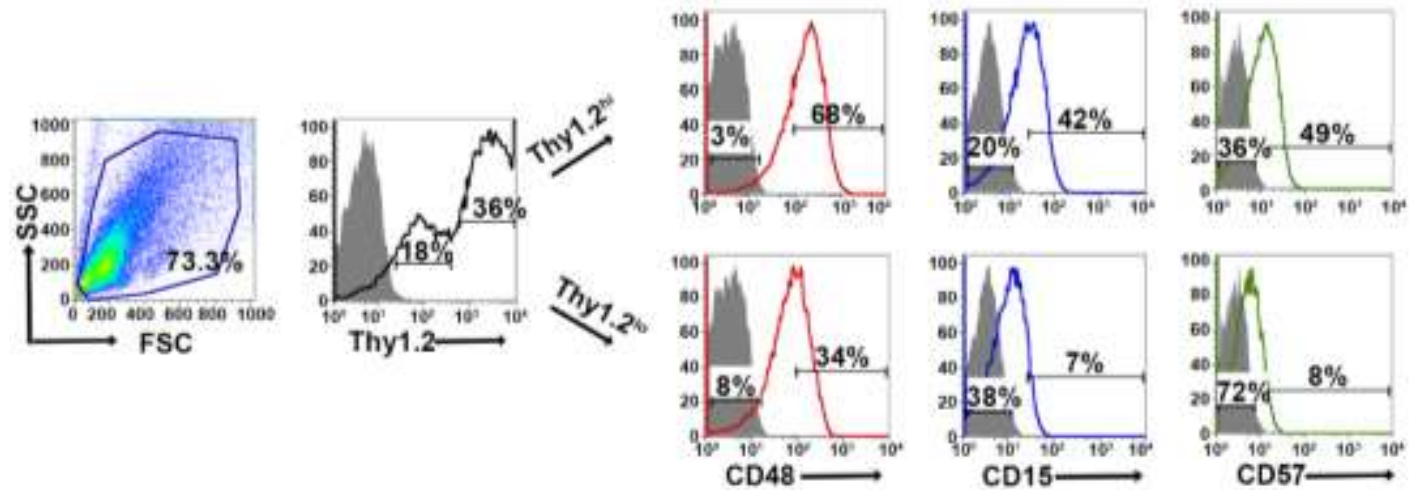

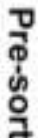

Figure 4-9. $\quad$ Surface marker expression of pre-sorted retinal cells

Far left panel: Live retinal cells show two distinct populations of Thy1.2 (Thy1.2 ${ }^{\text {hi }}$ and Thy $1.2^{\text {low }}$ ) based upon expression levels per cell. Right panels: Representative FACS plots show the expression of surface markers used for negative selection-CD48, CD15, CD57-in pre-sorted Thy1.2 $2^{\text {hi }}$ and Thy $1.2^{\text {low }}$ retinal cells. Labeled retinal cells were sorted for Thy $1.2^{\text {hi }} C D 48^{\text {neg }} C D 15^{\text {neg }} C D 57^{\text {neg }}$ population. Selection for sorting included the positive selection of Thy $1.2^{\text {hi }}(36 \%)$. This population was further selected for CD $48^{\text {neg }}$ (3\%), followed by CD15 ${ }^{\text {neg }}(20 \%)$ and CD57 $7^{\text {neg }}(36 \%)$. Grey indicates isotype controls, colored solid lines indicates experimental samples. 

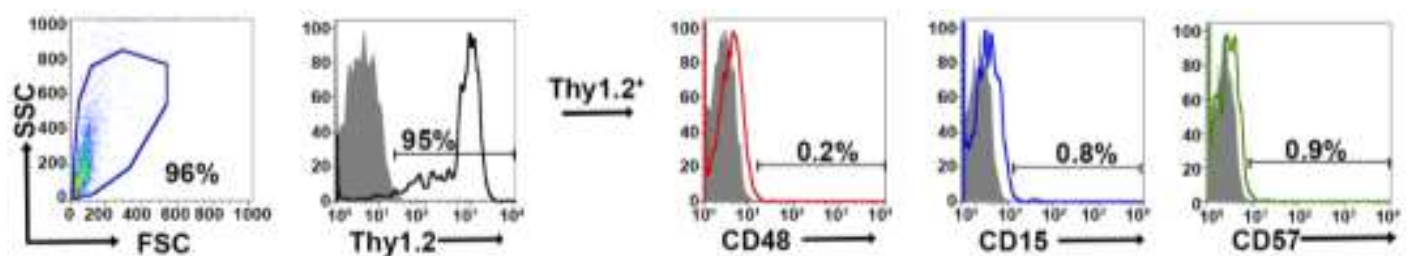

Intracellular staining - Live Thy $1.2^{+} \mathrm{CD} 48^{\text {nes }} \mathrm{CD} 15^{\text {reg }} \mathrm{CD} 57^{\text {neg }}$
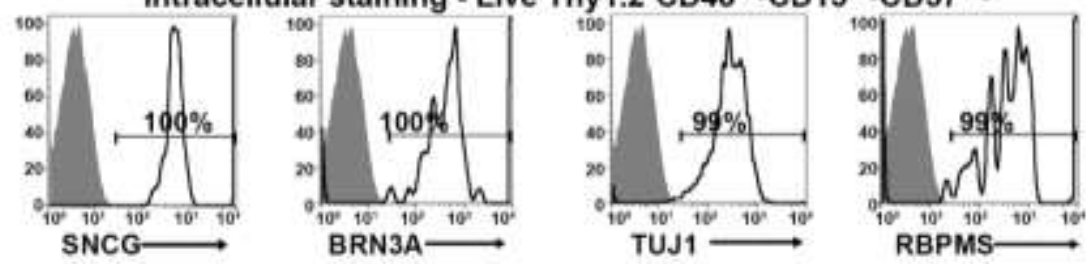

Figure 4-10. Purity of post-sorted RGCs based on the surface marker and intracellular RGC markers

Far left panel: LiveThy $1.2^{+} \mathrm{CD} 48^{\text {neg }} \mathrm{CD} 15^{\text {neg }} \mathrm{CD} 57^{\text {neg }}$ cells show expression of Thy $1.2^{+}$ (95\%). Top right panels present representative FACS plots show the negligible expression of surface markers in sorted LiveThy $1.2^{+} \mathrm{CD} 48^{\text {neg }} \mathrm{CD} 15^{\text {neg }} \mathrm{CD} 57^{\text {neg }}$ cells, demonstrating the efficiency of the sort. Lower row panels show representative FACS plots of the expression of intracellular RGC markers in LiveThy $1.2^{+} \mathrm{CD} 48^{\text {neg }} \mathrm{CD} 15^{\text {neg }} \mathrm{CD} 57^{\text {neg }}$ cells. $99-100 \%$ of all cells express SNCG, BRN3A, TUJ1 and RBPMS, all well characterized RGC markers. This new sorting method (Thy $\left.1.2^{\text {hi }} \mathrm{CD} 48^{\text {neg }} \mathrm{CD} 15^{\text {neg }} \mathrm{CD} 57^{\text {neg }}\right)$ shows improvement over the previously used methodology (LiveThy $1.2^{+} \mathrm{CD} 48^{\text {neg }}$ ) using additional surface markers. Grey indicates isotype controls, colored solid lines indicates experimental samples. 

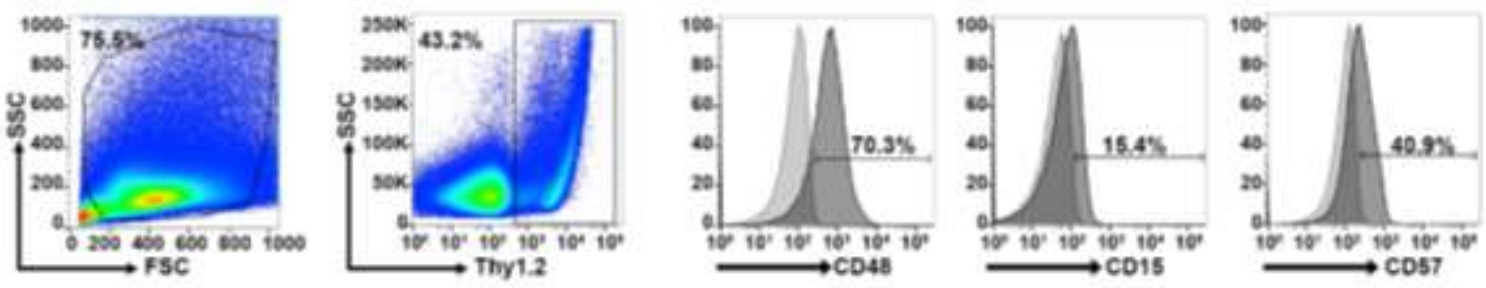

Figure 4-11. Consistency of flow cytometry results using MACSQuant ${ }^{\circledR}$

Dissociated murine retinal cells from C57BL/6J mice were labeled for cell surface markers based on the sorting protocol. Sample was acquired using a MACSQuant ${ }^{\mathbb{R}}$ Analyzer 10 from Miltenyi Biotec. Results show consistency between the two different cytometers (BD FACS Aria and MACSQuant ${ }^{\mathbb{B}}$ ).
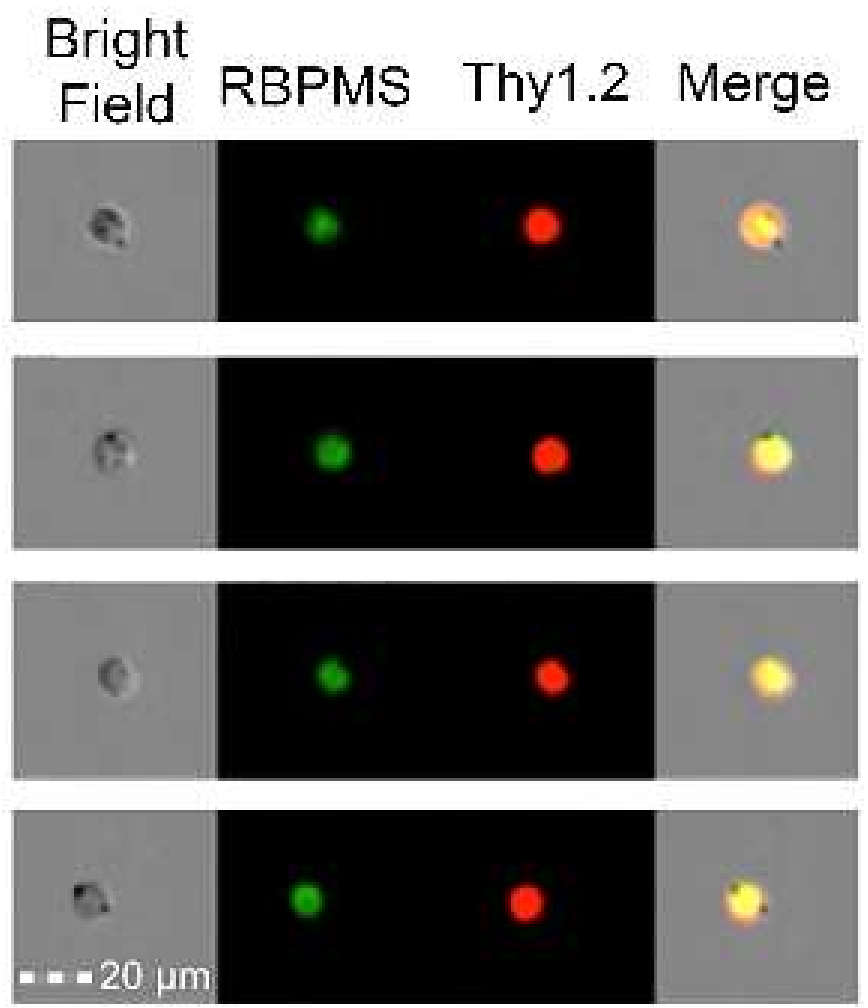

Figure 4-12. Intracellular expression of the RGC marker RBPMS in Live

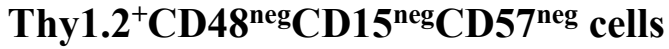

Representative images from a cohort of 250,000 pictures at $20 \mathrm{x}$ of cells using the FlowSight ${ }^{\circledR}$ Imager. Results show the expression of Thy1.2 surface and RBPMS intracellular markers. 

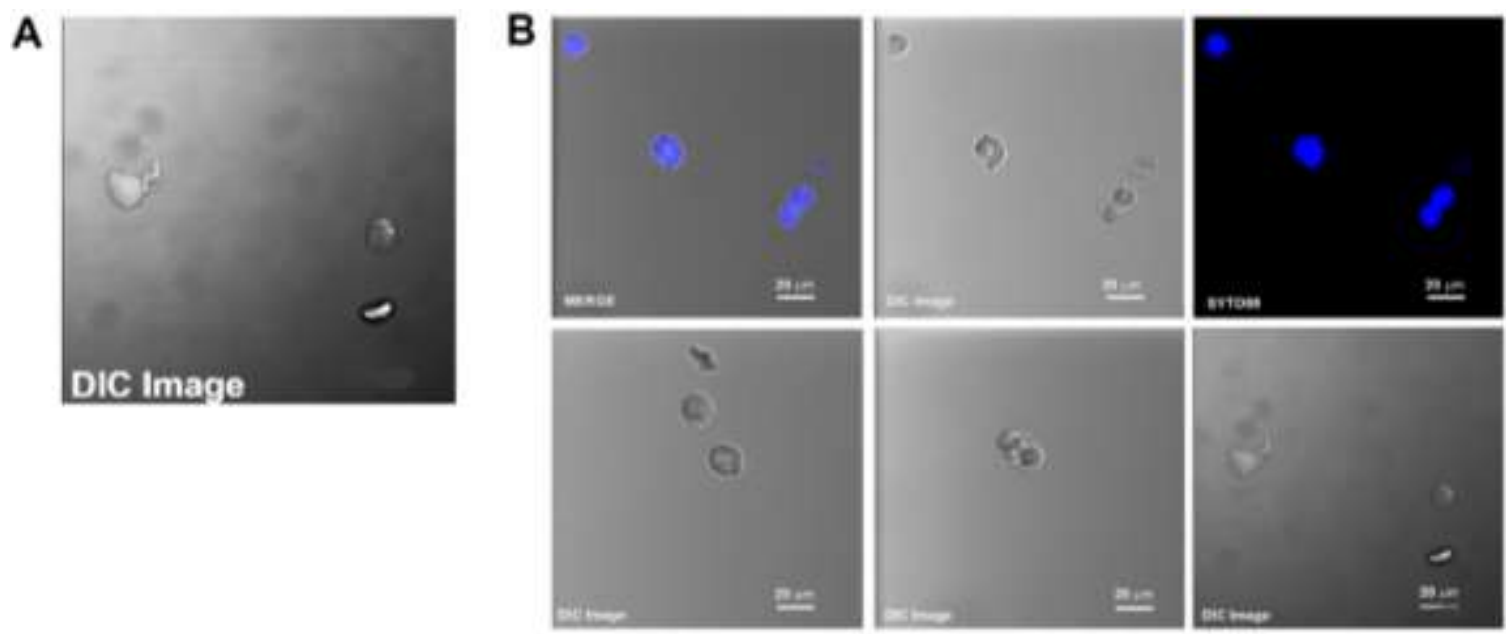

Figure 4-13. Live Thy $1.2^{+} \mathrm{CD} 48^{\text {neg }} \mathrm{CD}^{\mathrm{n}}{ }^{\text {neg }} \mathrm{CD57}^{\text {neg }}$ show RGC morphology

(A) Representative image of sorted cells after cultured overnight at $37^{\circ} \mathrm{C} / 5 \% \mathrm{CO}_{2}$ in $\mathrm{RGC}$ culture media showing RGC-type morphology. (B) Representative images of sorted cells, immediately after sort. 


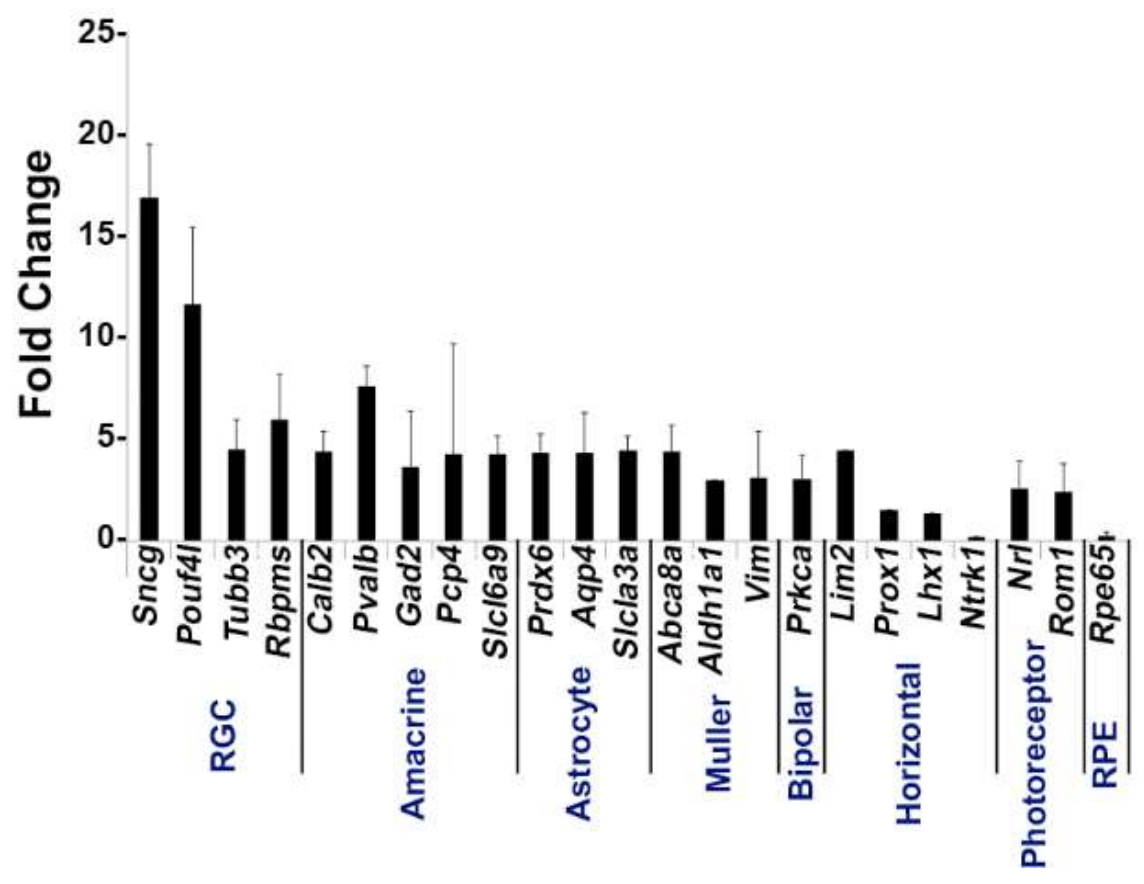

Figure 4-14. Validation of Live Thy $1.2^{+} C 48^{\text {neg }} C D 15^{\text {neg }} C D 57^{\text {neg }}$ cells using qPCR analyses

Graph depicts expression of different retinal cells markers in Live Thy $1.2^{+} \mathrm{CD} 48^{\text {neg }} \mathrm{CD} 15^{\text {neg }} \mathrm{CD} 57^{\text {neg }}$ that were normalized to the mRNA expression measured in total primary murine retinal cells. Genes associated with RGCs-Sncg, Pouf41, Tubb3 and Rbpms - showed increased gene expression or mRNA transcripts compared to non-RGC associated genes. Target gene expression levels are presented as $\log _{2}$ fold change based on $\mathrm{C}_{\mathrm{T}}$ calculation using Hprt as housekeeping gene. Mean \pm $\mathrm{SEM} ; \mathrm{n}=3$ biological replicates were performed in triplicate. 


\section{Highly enriched RGCs can be isolated from a mouse model with elevated IOP and optic nerve damage}

We sought to apply our improved RGC isolation methodology on a retinal degenerative disease model. Members of our research group have analyzed a family of over $100 \mathrm{BXD}$ (68) murine strains and their parental strains - C57BL/6J and DBA/2J-at 5 different age cohorts to determine which strains had the phenotype of optic nerve damage and elevated IOP (IOP). Both of these phenotypes are associated with retinal degeneration in glaucoma. Our examination revealed that the BXD66 strain had both elevated IOP and higher optic nerve damage compared to both C57BL/6J and DBA/2J parental strains of mice (Figure 4-15). In addition, the optic nerves of old BXD66 mice had an increase in axon damage and glial scarring compared to young C57L/6J and BXD66 mice. Figure 4-16 depicts representative micrographs showing optic nerve damage.

To determine if our RGC protocol was valid using retinae with damaged RGCs, we applied our optimized and validated enrichment strategy on retinae obtained from young and old BXD66 mice. We compared two different ages to investigate if our enrichment protocol could be used on mice with compromised RGCs and if the efficiency of the strategy was age dependent. We first compared the cellularity of retinal cells and the percentage of live cells between the two ages of mice. Selecting for the phenotype LiveThy1.2 $2^{+} \mathrm{CD} 48^{\text {neg }} \mathrm{CD} 15^{\text {neg }} \mathrm{CD} 57^{\text {neg }}$, enriched RGCs (Figure 4-17) were examined for the presence of the same signature intracellular RGC markers that we used throughout this investigation. Figure 4-18 shows the intracellular staining pattern in young and old BXD66 mouse retinas. Similar to that found in C57BL/6J mice, the majority of the enriched cells from BXD66 mice are immunoreactive toward the four intracellular RGC markers (Figure 4-19). However, we observed a slight reduction in TUJ1 in BXD66 young and old mice compared to the $\mathrm{C} 57 \mathrm{BL} / 6 \mathrm{~J}$ parental line, which may be indicative of RGC damage in this model. When comparing the total number of retinal cells (obtained from retinae of young and old C57BL/6J and BXD66 mice) with those of the phenotype LiveThy $1.2^{+} \mathrm{CD} 48^{\text {neg }} \mathrm{CD} 15^{\text {neg }} \mathrm{CD} 57^{\text {neg }}$, we found similar patterns of changes (compares both panels in Figure 4-20A). Within a strain, retinae from old mice had fewer live retinal cells than young mice. In addition, retinae from BXD66 mice had a reduced number of retinal cells compared to age-matched C57BL/6J mice (Figure 4-20B). Moreover, old C57BL/6J mice had a lower percentage of Thy $1.2^{\text {hi }} \mathrm{CD} 48^{\text {neg }} \mathrm{CD} 15^{\text {neg }} \mathrm{CD} 57^{\text {neg }}$ retinal cells compared to young mice $(0.9 \% \pm 0.3$ in young mice compared to $0.5 \% \pm 0.3$ in old mice, $p=0.041$, Figure 4-20B). We consistently found a marked reduction in the number of live retinal cells between BXD66 old $(>12$ months old) and young (5 weeks old) mice (Figure 4-20; live cells young versus old: $80 \%$ versus $48 \%$ ). It is worth noting that the percentage of live cells isolated from young pre-degenerative BXD66 mice is comparable to that obtained from young C57BL/6J mice (compare to Figure 4-2). Retinae from young BXD66 mice also had a higher, but not significantly different, percentage of Thy $1.2^{\text {hi }} C D 48^{\text {neg }} C D 15^{\text {neg }} C D 57^{\text {neg }}$ cells compared to old BXD66 mice $(0.4 \% \pm 0.3$ in young mice compared to $0.2 \% \pm 0.2$ in old mice, $p=0.177)$. Lastly, there was a significant reduction in the percentage of 


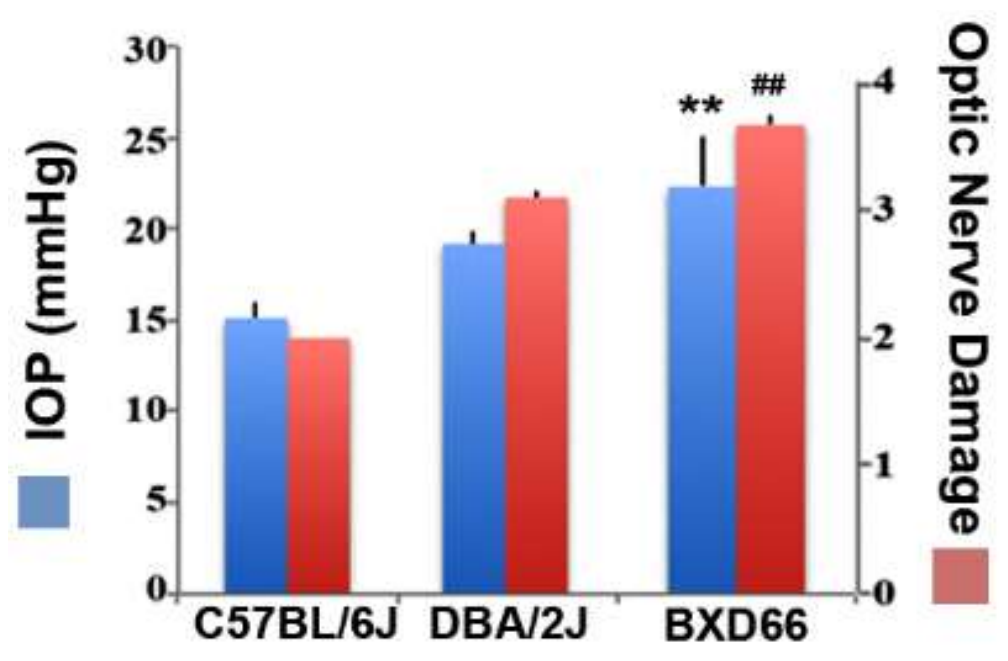

Figure 4-15.

Fewer RGCs are harvested from mice with documented elevated IOP and optic nerve damage

Peak IOP and optic nerve damage distributions for C57BL/6J, DBA/2J and BXD66 mice. BXD66 mice had significantly higher peak IOP (blue bars) and optic nerve damage (pink bars) compared to C57BL/6J mice (** $<<0.005$ between B6 and BXD66 IOP values; \#\# $p<0.005$ between B6 and BXD66 optic nerve damage grade). Mean $\pm \mathrm{SEM} ; n=4$ per group.

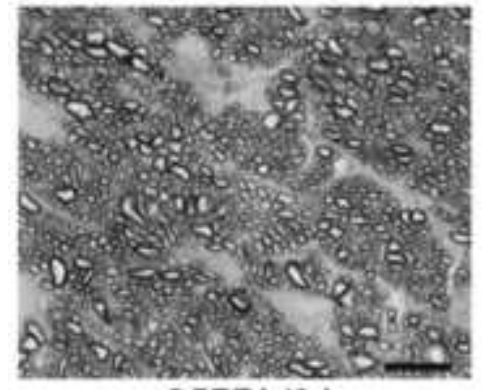

C57BL/6J

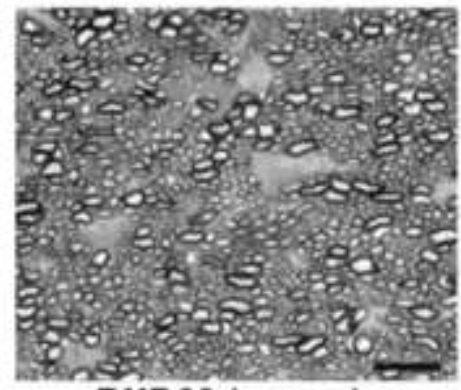

BXD66 (young)

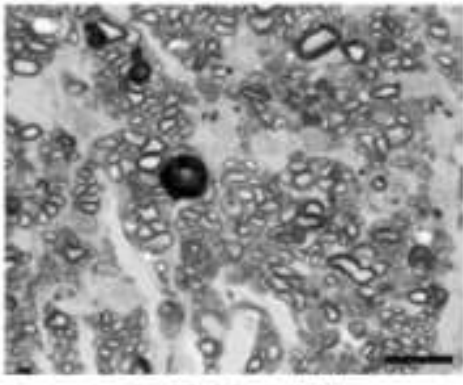

BXD66 (old)

Figure 4-16. Optic nerve damage in C57BL/6J and BXD66 young/ old mice

Representative PPD-stained optic nerve cross-sections from C57BL/6J (young: 5-7 weeks old), BXD66 (young: 5 weeks old), and BXD66 (>12 months old) mice. Both young $\mathrm{C} 57 \mathrm{BL} / 6 \mathrm{~J}$ and BXD66 mice had a low degree of damage. In contrast, optic nerves from old BXD66 mice presented with disorganized axon bundles, increased glial scarring and multiple dead/dying axons. Scale bar $=10 \mu \mathrm{m}$. 


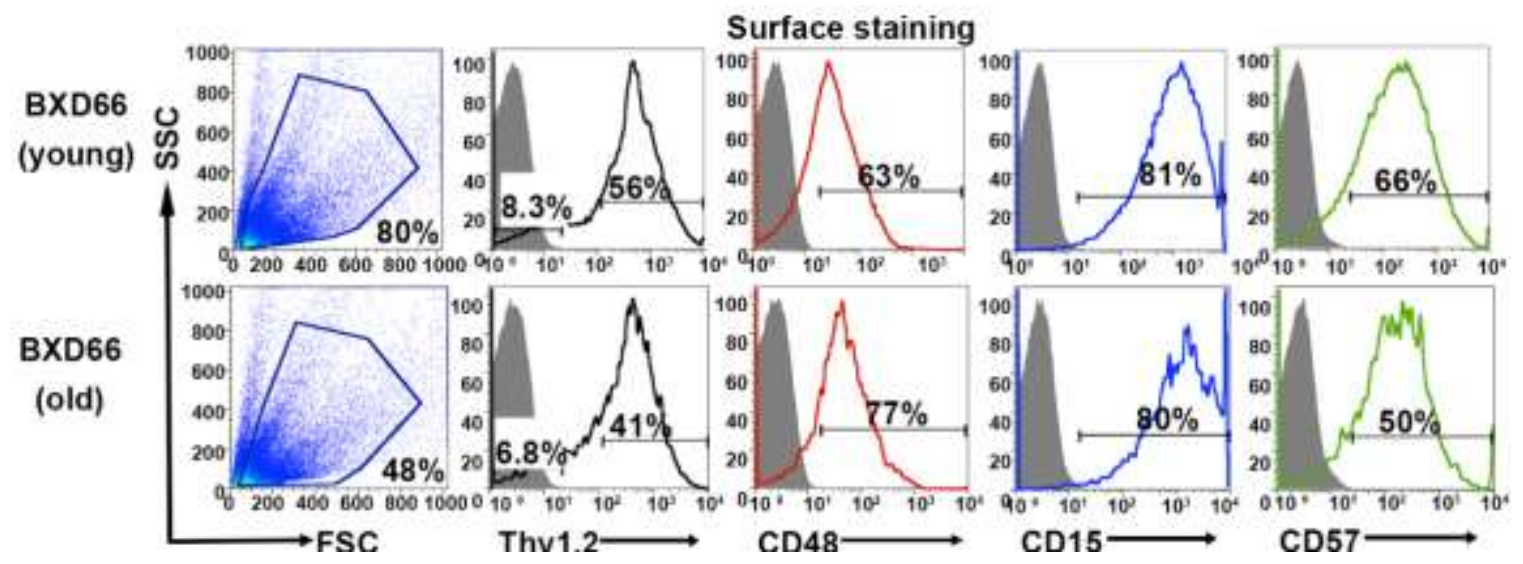

Figure 4-17. Characterization of enriched RGCs from young and old BXD66 mice obtained through our improved flow sorting method

Representative histograms show comparison of surface expression of Thy1.2, CD48, CD15 and CD57 in total retinal cells of young versus old BXD66 mice. Top panel: In BXD66 young mice, live retinal cells have the following abundance levels: Thy 1.2 (56\%), CD48 (63\%), CD15 (81\%) and CD57 (66\%). Bottom panel: In BXD66 old mice, live retinal cells have the following abundance levels: Thy1.2 (41\%), CD48 (77\%), CD15 $(80 \%)$ and CD57 (50\%). Gray histogram indicates isotype controls; black solid lines indicates antibody labeling. 


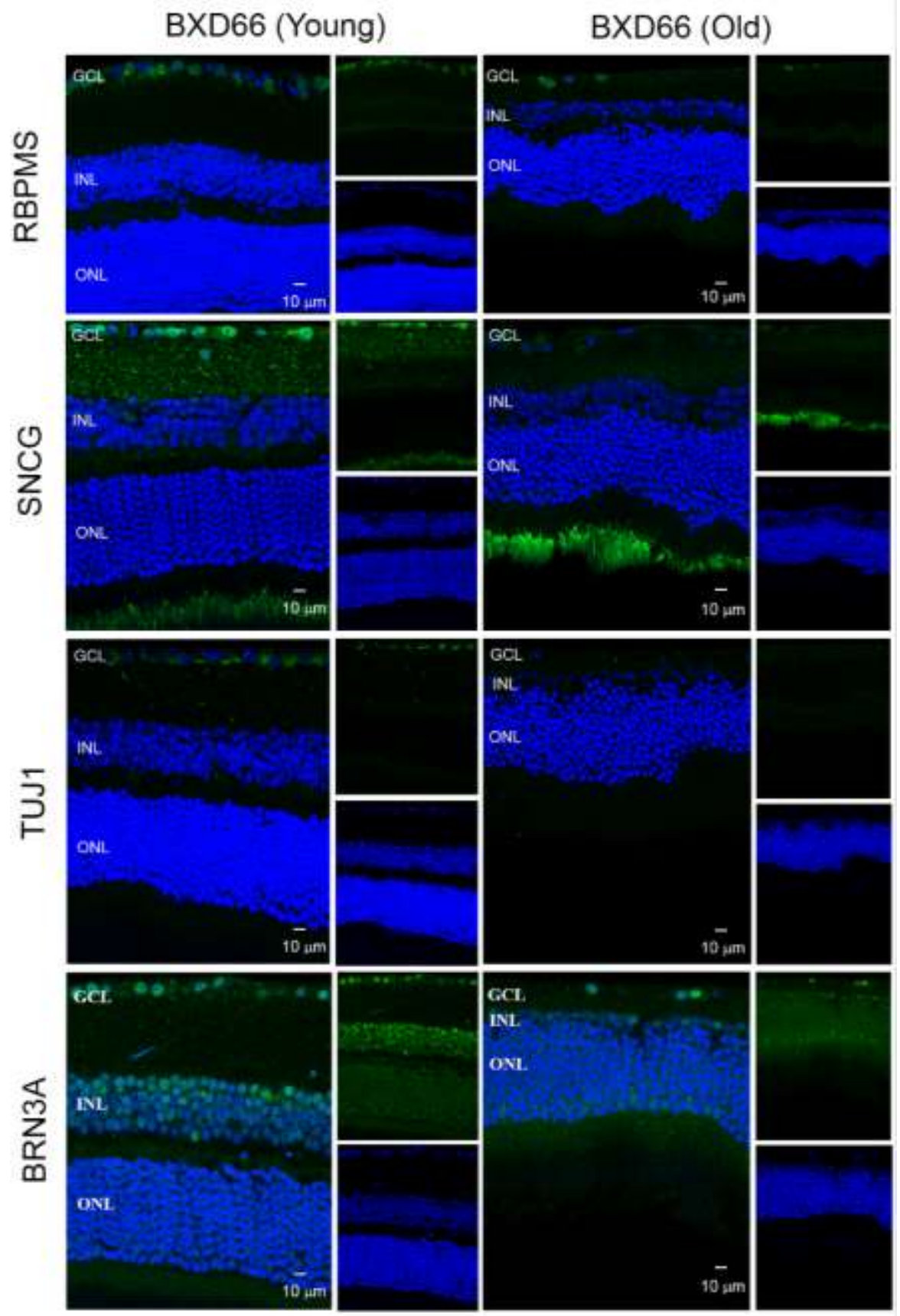

Figure 4-18. Localization of known intracellular RGC markers in BXD66 young/old mice

SNCG, BRN3A, TUJ1 and RBPMS in retinae from young and old BXD66 mice.

Sections from young and old BXD66 mouse retinae were labeled with antibodies against (C) SNCG, (D) BRN3A, (E) TUJ1, and (F) RBPMS. TO-PRO-III iodide staining labeled nuclei of all retinal cells (blue). Abbreviations: $\mathrm{GCL}=$ ganglion cell layer; $\mathrm{IPL}=$ inner plexiform layer; $\mathrm{INL}=$ inner nuclear layer; $\mathrm{OPL}=$ outer plexiform layer; $\mathrm{ONL}=$ outer nuclear layer. Scale bar: $10 \mu \mathrm{m}$. 


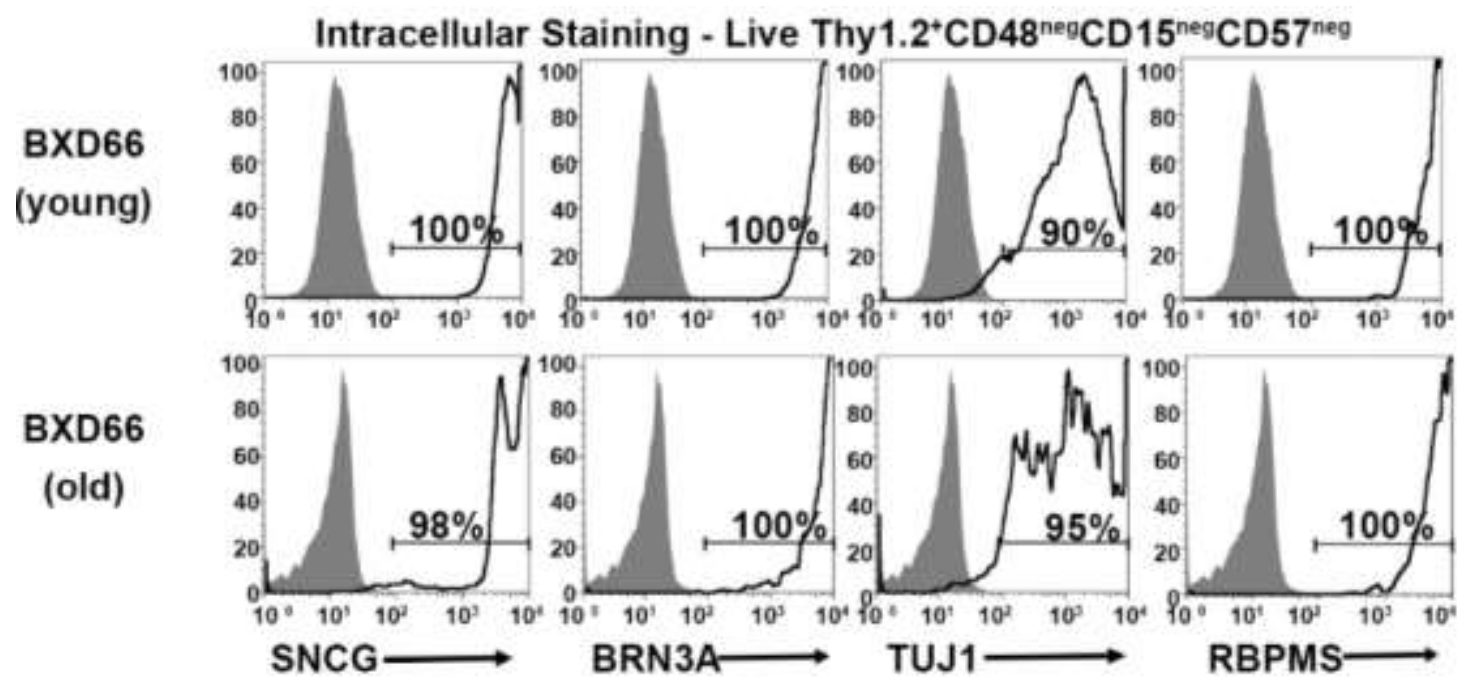

Figure 4-19. Relative purity of sorted RGCs based upon surface marker selection and intracellular RGC markers

Representative histograms show comparison of intracellular expression of RGC markers SNCG, BRN3A, TUJ1 and RBPMS in Live Thy1. $2^{+} \mathrm{CD} 48^{\text {neg }} \mathrm{CD} 15^{\text {neg }} \mathrm{CD} 57^{\text {neg }}$ cells of young and old BXD66 mice. Top panel: In BXD66 young mice have the following abundance levels: SNCG (100\%), BRN3A (100\%), TUJ1 (90\%) and RBPMS (100\%). Bottom panel: In BXD66 old mice have the following abundance levels: SNCG (98\%), BRN3A (100\%), TUJ1 (95\%) and RBPMS (100\%). Gray histogram indicates isotype controls; black solid lines indicates antibody labeling. 

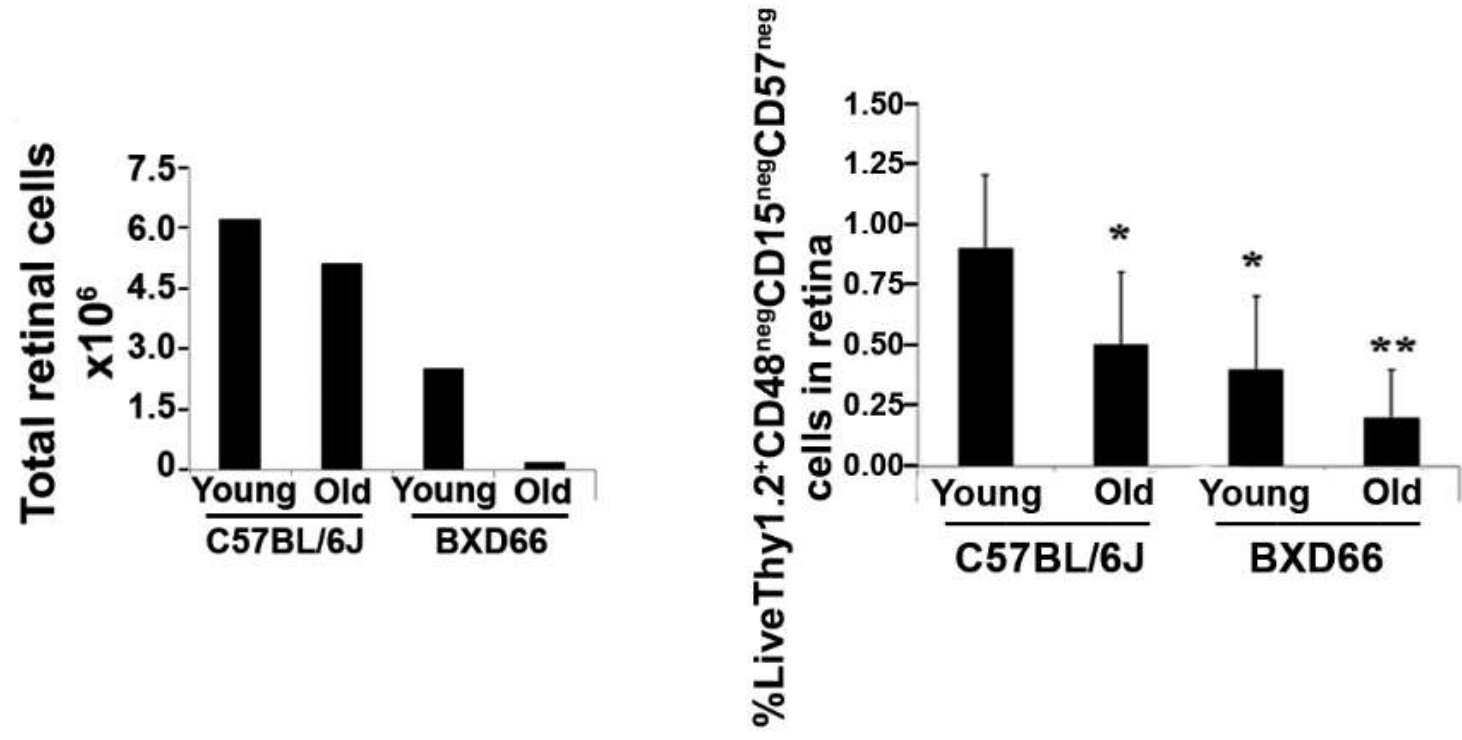

Figure 4-20. Comparison of live retinal cells based on sorting protocol

Percentage of Live Thy $1.2^{+} \mathrm{CD} 48^{\text {neg }} \mathrm{CD} 15^{\text {neg }} \mathrm{CD} 57^{\text {neg }}$ cells in retinae from C57BL/6J (young and old) and BXD66 (young and old) mice. $* * p<0.005 ; * p<0.05$ compared to young $\mathrm{C} 57 \mathrm{BL} / 6 \mathrm{~J}$ mice. Mean $\pm \mathrm{SEM}$; $\mathrm{n}=3$ per group. 
compared to $0.4 \% \pm 0.3$ in BXD66 mice, $p=0.024$ ). Collectively, our data show that our improved RGC enrichment protocol is also effective on retinae from old mice with no retinal degeneration $(\mathrm{C} 57 \mathrm{BL} / 6 \mathrm{~J})$ and in mice with a phenotype of elevated IOP and RGC damage (BXD66).

\section{Discussion}

A common element of the pathophysiology of several retinal neurodegenerations is the loss of ganglion cells and their axons (14). Understanding of the mechanisms underlying these processes is limited by the lack of a simple in vitro system with which to study RGC function. This is in part is due to the scarce number of RGCs and the heterogeneity present among the current cellular enrichment protocols. The use of antibody-dependent plate adhesion, or immunopanning, originated more than 35 years ago when it was used for the enrichment of immune cells. Barres and colleagues (209) utilized the immunopanning system wherein they used Thy1 and CD48 to isolate RGCs. They demonstrate the following three points: 1) Thy1 is not an exclusive marker of murine or rat RGCs, as other Thy $1^{+}$retinal cells do not show the morphology and/or electrophysiological characteristics of RGCs; 2) there is variation in the expression of Thyl positivity among RGCs; and 3 ) this assay only produced a 95\% efficiency based on Thyl expression. This classical study allowed for the first time the use of immune techniques in the field of neuroscience. Prior to the publishing of this methodology, RGC isolation was based on density gradient centrifugation with varying yield degrees (226228). Although inexpensive and rapid, a major drawback of Barres's methodology was that it required the retrograde labeling of fast blue into both superior collicular and brachia 48-hrs prior to retinal dissection. In 1999, Shoge and colleagues (229) developed a protocol for the enrichment of rat RGCs based on magnetic cell separation via inclusion of Thy $1^{+}$cells and the exclusion of macrophages. This method became popular because it provided a fast method for enrichment of rat RGCs, although it required magnetic columns and magnetic drivers for the separation. Unfortunately, their RGC enrichment was only $31 \%$ indicating that many contaminating cells are present. Recently, Hong and colleagues (210) combined the immunopanning and magnetic cell sorter techniques to isolate murine postnatal (P1-4) RGCs. However, because the success of the method was based solely on exclusion of glial fibrillary acidic protein and synthaxin 1, the purity of the RGCs that were isolated is unknown.

We sought to develop an improved RGC enrichment strategy that could be used by most investigators without the requirement for retrograde labeling that may alter the physiology of RGCs. Our protocol also does not include immunopanning which is lengthy. Our method is rapid and requires only five hours from the initiation of retinal dissection to the completion of fluorescence activated cell sorting. Because of the exquisite sensitivity of the method, FACS-based sorting is suited for purification of cells that comprise a very small percentage within a cell suspension. Thus, the procedure is ideal for the isolation and enrichment of RGCs, estimated to be about $0.5 \%$ of retinal cells $(148,204)$. A caveat of our methodology is the requirement of expensive FACS instrumentation, and the need for highly trained and specialized operator. However, most 
academic and research facilities have flow cytometry core facilities, which should make this method accessible to most investigators. FACS-based sorting has a tremendous versatility because of the large number of selection markers that can be used in the enrichment process. Typically, the selection is based on 2 to 15 complementary parameters, which allow for acquisition of a reproducible and homogeneous phenotype within a sample and between samples that can be used in follow up in vitro studies. This methodology also allows for the immediate identification of viable cells and excludes cells that are undergoing apoptosis or are already dead. In addition, the inclusion of an $\mathrm{Fc}$ receptor blocker allows for exclusion of microglia, monocytes and macrophages, which results in a higher degree of RGCs. Post-sorting analysis of enriched RGCs indicated cell viability was $95 \%$, confirming that external high pressure (60-70 psi) exerted on cells during FACS had a negligible effect on their viability. Lastly, another advantage of this methodology is the immediate verification of the sort purity, as it only takes as small aliquot of the isolated product to verify the surface phenotype.

As part of our RGC enrichment strategy, we incorporated robust validation components. For example, within our validation studies, we use immunohistochemistry to validate two additional surface markers-CD15 and CD57-that improved the efficiency of our sorting strategy. In addition, the stringency of FACS validation strategy was confirmed using four RGC specific intracellular markers-Sncg, Pouf4l, Tubb3, and Rbpms, which encode for SNCG, BRN3A, TUJ1, and RBPMS, respectively - both at the protein and mRNA level. Furthermore, we identified the presence of non-RGCs using multiple genes expressed by other retinal cell types using qPCR analyses. To our knowledge, this is the first time, such a stringent validation process using both gene expression (qPCR) and protein (flow cytometry and immunohistochemistry) analyses have been performed on isolated and enriched murine RGCs.

As a further validation of the applicability of our enrichment protocol, we evaluated the ability of our RGC enrichment strategy to isolate RGCs from a mouse model with elevated IOP and optic nerve damage. Members of our collaborative group use the BXD family of RI mice for gene mapping and quantitative trait locus QTL analyses to identify specific genomic regions that modulate various glaucoma-associated endophenotypes $(59,82,230)$. The BXD RI strains are derived by inbreeding the $\mathrm{C} 57 \mathrm{BL} / 6 \mathrm{~J}$ and DBA/2J parental strains. BXD mice have been successfully used in vision research to elucidate specific cause-effect predictions between genes and a quantitative phenotype, such as differences in expression levels $(68,70,82,119)$. In these studies, we selected the BXD66 strain due to its age-dependent elevation in IOP and optic nerve damage (not published but available on www.GeneNetwork.org). Our intracellular flow cytometry protein analysis revealed a similar percentage of cells that show positivity for the surface marker Thy1.2, while negative selection of CD48, CD15 and CD57, and concomitant positivity for the intracellular markers-SNCG, BRN3A, TUJ1, and RBPMS - in both C57BL/6J mice and BXD66, irrespective of the age bin (5 weeks versus 12 months). Nonetheless, the percentage of RGCs was significantly lower in the BXD66 mice compared to the C57BL/6J mice, suggesting that RGCs, a population that is already scarce in the mammalian retina, is less abundant in BXD66 mice. Our data also highlight variation in the expression of TUJ1 in RGCs isolated from BXD66 mice at 
different ages, suggesting that differences in TUJ1 levels likely reflect altered physiology of the damaged RGCs, rather than the absence of RGCs in these mice.

In summary, we demonstrate a powerful technique for the isolation and enrichment of primary murine RGC with the phenotype Live Thy $1.2^{\text {hi }} \mathrm{CD} 48^{\text {neg }} \mathrm{CD} 15^{\text {neg }} \mathrm{CD} 57^{\text {neg }}$, which concomitantly express the RGC signature intracellular markers SNCG, RBPMS, TUJ1, and BRN3A. These cells can be used for controlled in vitro studies of RGCs derived from healthy and disease models. The streamlined and effective isolation and validation method described here will facilitate subsequent research on the pathological and pharmacological processes with clinical relevance to diseases involving RGCs. 


\title{
CHAPTER 5. DISCUSSION AND CONCLUSION
}

\author{
Future Directions
}

\begin{abstract}
Aim1: To identify the candidate gene that modulates IOP in mouse and test translational validity of the mouse candidates using the cohorts of human glaucoma patients
\end{abstract}

The main characteristic feature of glaucoma is the progressive and relatively slow damage/death of RGCs (1). Ganglion cell death is also a feature of a number of other optic neuropathies and retinopathies apart from glaucoma. The known acute induced models that are currently used include, optic nerve crush, ischemia/reperfusion injury, excitotoxic damage etcetera; are useful only in some aspects for glaucoma research (231). The injury caused in these models is not just a specific damage to RGCs but a general injury to the whole retina, therefore these models resembles more closely to other diseases than glaucoma. To study such a progressive disease, the most relevant rodent glaucoma model should include chronic elevation of IOP, to study high tension glaucoma. Due to the difficulty in reproducing a standardized chronic elevated IOP model thus far in the rodents, a main focus of interest should be to discover rodent strains with spontaneously elevated IOP.

The mouse models with chronic IOP that are currently available are mostly knock out models with single gene mutations, such Mendalian mutations do not correlate with human glaucoma (231). In the past decade and a half dramatic advances has been seen in the field of glaucoma research due the introduction of chronic inherited disease in inbred mice strains. DBA/2J inbred line is the most well characterized mouse model of glaucoma (64). Although this model has been extensively studied, few limitations makes this model not ideal for studying glaucoma. In these animals, the glaucoma progression is not due elevated IOP. The disease progression is in fact due to the secondary systemic pigment dispersion syndrome with the associated mutations in the Gpnmb and Tyrpl loci. The natural history of disease in $\mathrm{DBA} / 2 \mathrm{~J}$ mice varies, depending on individual colonies and other environmental factors. Also, even though these are inbred mice with a fixed genetic background, they still exhibit a high degree of variability and asymmetry in developing the disease. Only about $70 \%$ of the animals develop glaucoma since both of these genes show incomplete penetrance $(66,67)$.

Because BXD mice are genetically stable inbred lines, this makes them an informative tool for understanding human disease. The combined BXD strain set is the largest existing mouse RI mapping panel. Using a systems genetics approach, we and other researchers have successfully used BXD RI murine panel for studying genetic modulators and identifying non-synonymous polymorphisms for complex polygenetic diseases such as glaucoma, pigment dispersion syndrome, hypertension, and metabolic disorders system (69-71). RI strains are especially useful for mapping complex traits, since they create an immortalized mapping population that allows us to phenotype as 
many animals per genome as desired over extended periods of time which leads to more precise phenotypic estimates invaluable to mapping complex disease traits as glaucoma. Using this information, we can identify specific wild type BXD strains (i.e., no Tyrpl and Gpnmb mutations) that have elevated IOP, high optic nerve damage and iris defects that can be used as high tension glaucoma model. We can also distinguish the strains based on IOP and optic nerve damage grades to identify normal tension glaucoma models. Such mouse models would be ideal to study glaucoma progression, since these mice will not have any influence of Tyrpl and Gpnmb genes, and natural variations due to recombination events would ideally represent human disease states such as POAG.

Genetic studies using BXD mice are proving to be a powerful means to study disease causing genes and pathologic mechanisms that cause disease or alter disease susceptibility. The systems genetics approach has been widely used to identify the genetic basis for pharmacogenetic traits and becomes increasingly more powerful with the recent advances in genomic technologies. We can easily study a genetic association with drug response if there is a plausible link between the gene and the drug response. We can characterize physiological or pharmacologic effects of the genes (or genetic variations) on disease or drug response. In spite of increased spending on pharmaceutical research and development, the number of 'new' drug approvals for glaucoma has remained relatively constant. The essential first step in the drug discovery pipeline is the identification and early validation of disease-modifying targets. In recent years 'systems' approach has become popular to identify new targets to understand the cellular mechanisms underlying a particular disease. The systems approach is geared towards target-discovery through the study of disease in whole organisms such as mice or rats (232). Here, we used 'systems genetics' to identify IOP-associated genes. In future we can validate these as functional targets, and utilize such approach in bridging the gap between data generation and effective drug target identification. In this study, the identification of a novel candidate-CACNA2D1-linked to elevated IOP was an exemplar to pave the way for improved drug development tailored to individuals with glaucoma leading to personalized medicine. The outcomes of these investigations will identify a novel therapeutic for IOP reduction that specifically targets a gene product that was identified in bidirectional POAG studies. They will also determine if the genotype influences response to therapy. Such results are expected to fundamentally advance the field of glaucoma therapy, which will have positive translational impact.

Our Aim 1 studies are innovative for at least the following five reasons:

1. We have utilized a large number of aging BXD strains that are segregating for glaucoma susceptibly genes. The DBA/2J strain has been used as the standard model for pigmentary dispersion glaucoma by a substantial number of investigators. We have greatly enriched this field by introducing new variant strains that have both Tyrpl and Gpnmb mutations and new strains with wild type genotypes that have a highly variable disease state. Our set of 66 aging BXD strains is sufficiently large to allow us to identify loci, and even genes, that cause glaucoma.

2. We used the complete sequence data for $\mathrm{DBA} / 2 \mathrm{~J}$ and the dense genotypes for all BXD strains. We now have a database of $~ 4 \mathrm{M}$ SNPs, $400 \mathrm{~K}$ indels, and thousands 
of inversions and CNVs that are segregating among the BXD strains. The consequence of this is that our QTL analysis and the massive genomic/genetic data led to more efficient extraction of functional sequence variants linked to IOP.

3. We used a novel systems genetics approach to help define molecular networks that modulate IOP. By using the eye transcriptome data and aging BXD IOP phenotype data, we were able to assemble gene-phenotype networks. This is one of the most exciting aspects and is a departure from the typical experimental paradigm of testing and comparing knockout and wildtype mouse models of human disease. This project represents a novel application of the use of expression genetics in dissecting the transcriptome predictors of IOP and other phenotypes associated with glaucoma.

4. We have combined mouse data sets with data from human GWA studies in a bidirectional manner to identify a candidate gene most likely to modulate IOP.

5. We provide the scientific community with a novel gene candidate-CACNA2D1- that influences IOP based upon a systems genetics approach by using BXD strains.

\section{Aim 2: To identify and validate regulator of $\mathrm{Sncg}$ in primary mouse RGCs}

Glaucoma is characterized by $\mathrm{ON}$ axon loss and RGC degeneration and is often linked with elevated IOP. In a complex disease such as glaucoma, the molecular processes driving the complex disease usually affects genes acting in concert. One of the strategies that we used for these studies was systems - level approaches to investigate transcriptional networks and pathways within pathologically relevant cells - RGCs. Our integrated analysis of transcriptional networks associated with $\mathrm{Sncg}$ susceptibility data and phenotypic information allowed us to identify specific pathways to be connected to disease states, and thereby can identify disease pathways and their genetic regulators as new targets for therapeutic intervention. We took the full advantage of the BXD RI strains in combination with a powerful combination of systems biology, microarray analysis, expression genetics, bioinformatics along with conventional techniques to study mechanism of gene regulation.

Although the molecular mechanisms responsible for RGC death is not well known, considerable evidence suggests the involvement of $\gamma$-synuclein as a component of RGC death. Recent evidence shows that mitochondrial dysfunction is present in some glaucoma patients. POAG patients have been reported to have increased levels of mtDNA mutations as well as deficiencies in complex-I driven ATP synthesis and respiration (233). Although evidence of mitochondrial dysfunction was reported in patients with glaucoma, there is no direct evidence that mitochondrial dysfunction contributes to the pathogenesis of glaucoma. In our study the interaction of $\gamma$-synuclein with mitochondria raises the intriguing possibility that these two components of RGC damage might have a synergistic inter-relationship that contributes towards the complexities of glaucoma. Possible mechanisms of such an interrelationship include $\gamma$-synuclein directly or indirectly impairing mitochondrial function, mitochondrial 
dysfunction exacerbating $\gamma$-synuclein levels, or, perhaps, $\gamma$-synuclein levels and mitochondrial dysfunction enhancing each other in a self-amplifying cycle, or, $\gamma$-synuclein influencing mitochondrial fission-fusion reaction. Intriguingly the ER is well connected, both physically and functionally, to the mitochondria allowing the signaling and exchange of metabolites between these two organelles. Since both $\gamma$-synuclein and prefoldin-2 act as chaperone proteins, their levels may block ER to Golgi membrane trafficking. These two chaperone molecules may also perturb microtubule transport which may lead to impairment of both the appropriate delivery and distribution of mitochondria within RGCs and their axons, particularly from the critical area of the synaptic terminals, as well as the disposal of potentially reactive oxygen species producing dysfunctional mitochondria. In future such studies will enhance our understanding of the pathophysiological mechanisms of mitochondrial dysfunction in glaucomatous neurodegeneration, and offer the scientific basis for identifying and developing new therapeutic strategies that could protect not only RGCs and their axons, but also the central visual pathway against glaucomatous damage.

Our Aim 2 studies are innovative for the following reasons:

1. Here, we combined cutting edge methodologies of systems genetics, bidirectional studies using multiple species, meta-analyses, immunohistochemistry, FACS sorting and gene knockdown studies to identify and validate the identity of a genetic modulator of $S n c g$, a gene that has been previously implicated in RGC death in glaucoma.

2. We exploited partial correlation analysis to solidify the relationship between Sncg and $P f d n 2$ in the mouse retina. The statistically significant partial correlation between $S n c g$ and $P f d n 2$ reflects gene-gene interactions, as well as a regulatory relationship between the genes.

3. We exploited the transcriptome data from both healthy and diseased eyes to evaluate the relationship between $S n c g$ and the candidate gene. In healthy retinas, the protein levels of both genes are significantly correlated in a positive manner. In contrast, the relationship between the genes appears to have changed to an inverse relationship with reduced $S n c g$ levels in glaucoma as the disease progressed.

4. Pfdn2 is located in the Qrrl on chromosome 1, which is a genomic region of unusually high gene density and contains major regulatory QTLs for various behavioral, metabolic, physiologic and immunological processes including diverse epileptic traits $(188,234)$. This region also controls the expression of a remarkably large number of genes, including genes that are associated with some of the classical traits that map to distal Chr 1 (e.g., seizure susceptibility).

5. Knocking down $P f d n 2$ expression in enriched primary murine RGCs significantly reduced the expression of $S n c g$, confirming that $P f d n 2$ regulates $S n c g$ expression in murine RGCs. The knock down studies were performed in vitro and the cells remained alive for 48 hours post-transfection. 
6. The findings presented in this study provide strong support for the value of systems genetics, especially GeneNetwork, in discovering new upstream regulators of genes that can be confirmed by molecular analysis.

\section{Aim 3: To isolate and validate primary murine RGCs from healthy and recombinant inbred mouse model of elevated IOP}

Glaucoma is a complex, multifactorial, neurodegenerative disease that causes irreversible vision loss. A universal element in the pathophysiology of the many etiologies of glaucoma is the death of RGCs and the degeneration of their axons (1). Identifying the genetic basis or cellular mechanisms causing RGC degeneration would be the first step toward the development of efficacious therapies to slow or reverse RGC damage, in turn preserving vision. But scarce number of RGCs that can be isolated from a retina provides a major challenge to develop therapies.

A great deal has been learned about the cell and molecular biology of glaucoma through research on cultured RGCs. However, there are significant limitations with current RGC cultures. RGCs are terminally differentiated, and therefore, primary RGCs must be prepared from rodent eyes for each experiment conducted. A number of laboratories have emphasized the need for having a verified and validated RGC cell line to determine the mechanism of RGC cell death or to evaluate therapy efficacies (202, 203, 235). In attempt to address this need, in 2001, a rat retinal cell line (RGC-5) displaying certain characteristics of RGC (expression of the specific cell-type markers including Thyl, Brn-3C, neuritin, NMDA receptor, GABA $\beta$ receptor, and synaptophysin) was derived by transforming postnatal day 1 rat retinal cells with $\Psi_{2}$ EA1 virus (236). Multiple questions have been raised regarding the nature and/or identity of these cells. A 2009 study demonstrated that the RGC-5 cell line contained mouse, rather than rat, biomarkers (203). As recent as 2013, it was (235)demonstrated that RGC-5 cells were $661 \mathrm{~W}$ cells, a mouse SV-40 T antigen-transformed photoreceptor cell line, rather than rat RGCs. There have been more than 230 publications that have used the RGC-5 line to test various glaucoma-related hypotheses (237). Unfortunately, the outcomes of those investigations are very likely null and void. The lack of an authenticated RGC line represents a large unmet need for the vision research community at large. The heterogeneity of the cells in retina contributes to lack of reproducibility to isolate homogeneous RGCs. Therefore, to investigate RGCs cellular mechanisms, it is critical to develop such methodology. Commercially available kits for RGC isolation are exclusive for rats and for mice the protocols are based on magnetic separation and immunopanning. Both approaches require lengthy isolations and their yields vary. FACS is a powerful technique for analysis of cells in suspension allowing separation of cells based on parameters such as size and granularity, providing a quantitative and qualitative analysis with high sensitivity. The purification system using flow cytometry has not been standardized for the isolation of and standardization of primary murine RGCs from dissociated retinae. We can use this powerful technology — FACS — as the first step of generating a stable, immortalized, homogeneous murine RGC population from murine retinae. 
Classically, IHC analyses of cross sections of ganglion cell layer flat mounts are used for the detection of surface and intracellular markers associated with RGC (207, 208, 238, 239). Despite its extensive use there are a series of limitations such as fading and photobleaching of fluorescence, limited sensitivity and the presence of false-negative or false-positive results. The tissue structure may or may not allow appropriate exposure for the immune binding of proteins. Rather than the use of IHC for protein detection exclusively, the use of flow cytometry as a complement technique will provide a major sensitivity and a faster and more objective way to acquire and record data.

Enhancing RGC viability (neuroprotection) or RGC function (neuroenhancement) remains a major goal of basic and translational research. In future, such cell lines or isolate cells can be used to define the molecular biology of the process of ganglion cell death, by manipulating such biochemical events that occur in a dying ganglion cell to increase the chance of cell survival. Elucidation of cell death and neuro-protective pathways would lead to generation of novel therapeutic targets to prevent glaucoma progression. Other possibilities include, molecular profiling to identify and characterize the RGC subtypes which are the first targets of the disease - the cells most susceptible to the disease - and help devise new prevention and therapy. RGC-based screening technologies can be developed to enabled drug discovery scientists to develop models of interconnected cellular pathways, extract important information for specific disease models, and build companion diagnostic programs around relevant cellular biomarkers for therapeutics. The benefits from this work will pave the way for studying mechanisms of RGC neuroprotection and death in glaucoma and in future targeted neuroprotective strategies can be developed to treat glaucoma.

Our Aim 3 studies are innovative for the following reasons:

1. We provide the first standardized method for the enrichment of adult murine primary RGC by FACS.

2. LiveThy $1^{+} \mathrm{CD} 48^{\text {neg }}$ phenotype is not the phenotype of a homogenous/pure RGC population. The stringency of the additional positive markers: Thy1.2 and negative cell surface: CD15, CD57, CD48 selection markers increased the homogeneity of the resultant RGC population.

3. Our two step validation strategy included the use of qPCR and flow cytometry. The innovation is based on (1) pre-amplification od cDNA; (2) the combination of amplicons; (3) intracellular protein examination by flow cytometry. Our cutting edge gene transcriptional assays based on characterized the homogeneous population of the isolated cells and our flow cytometry strategy affirmed the purity of the isolated cells.

4. Our sorting method enriches for a homogeneous population of RGCs.

5. We provide the scientific community with a novel and fully validated methodology to isolate murine RGCs that can be used to dissect molecular mechanisms of cell damage/death in glaucoma as well as evaluate potential treatments for glaucoma. 


\section{Glaucomics_Future of Glaucoma Research}

Visual disorders like glaucoma massively impact health, both in developed and developing countries. Visual disorders represent one of the largest health care expenditures in the United States, costing approximately $\$ 139$ billion per year (240). A complex disease such as glaucoma is best conceptualized as a syndrome displaying a presumably common clinical end-point, but with heterogeneous molecular underpinnings and host-environment interactions. Despite advances in our understanding of the pathophysiology of glaucoma, the diagnostic criteria and associated endophenotypes are still evolving. Ophthalmology in the 21 st century needs a fresh new look at the ways in which novel diagnostics are developed so as to catalyze true innovations for personalized healthcare. This thesis is an introduction of omics science and systems diagnostics to ophthalmology, a field that can benefit enormously from personalized medicine, and which has sadly lagged behind in systems diagnostics compared to fields such as oncology. Glaucomics offers tremendous opportunities to integrate glaucoma research and clinical practice with omics science, systems diagnostics, and personalized visual health. Glaucomics can be defined as "The integrated use of multi-omics and systems science approaches towards rational discovery, development, and tandem applications of diagnostics and therapeutics, for glaucoma specifically, and for personalized visual health" (241). Access to high-throughput technologies has enabled scientists and clinicians to examine genomes, transcriptomes, proteomes, metabolomes, and other omics information. By combining mouse and human omics data we can identify novel biomarkers and systems diagnostics for glaucoma. By isolating different retinal cell types contributing to glaucoma we can perform high throughput drug screening and high content data analysis to better understand cellular function (Figure 5-1). My dissertation is a step towards development of "Glaucomics" as a bridge between high throughput technology and visual health. Philosophers defined the eye as a window to the soul long before. Now as scientists we need address this cliché to determine its scientific basis and clinical relevance. 


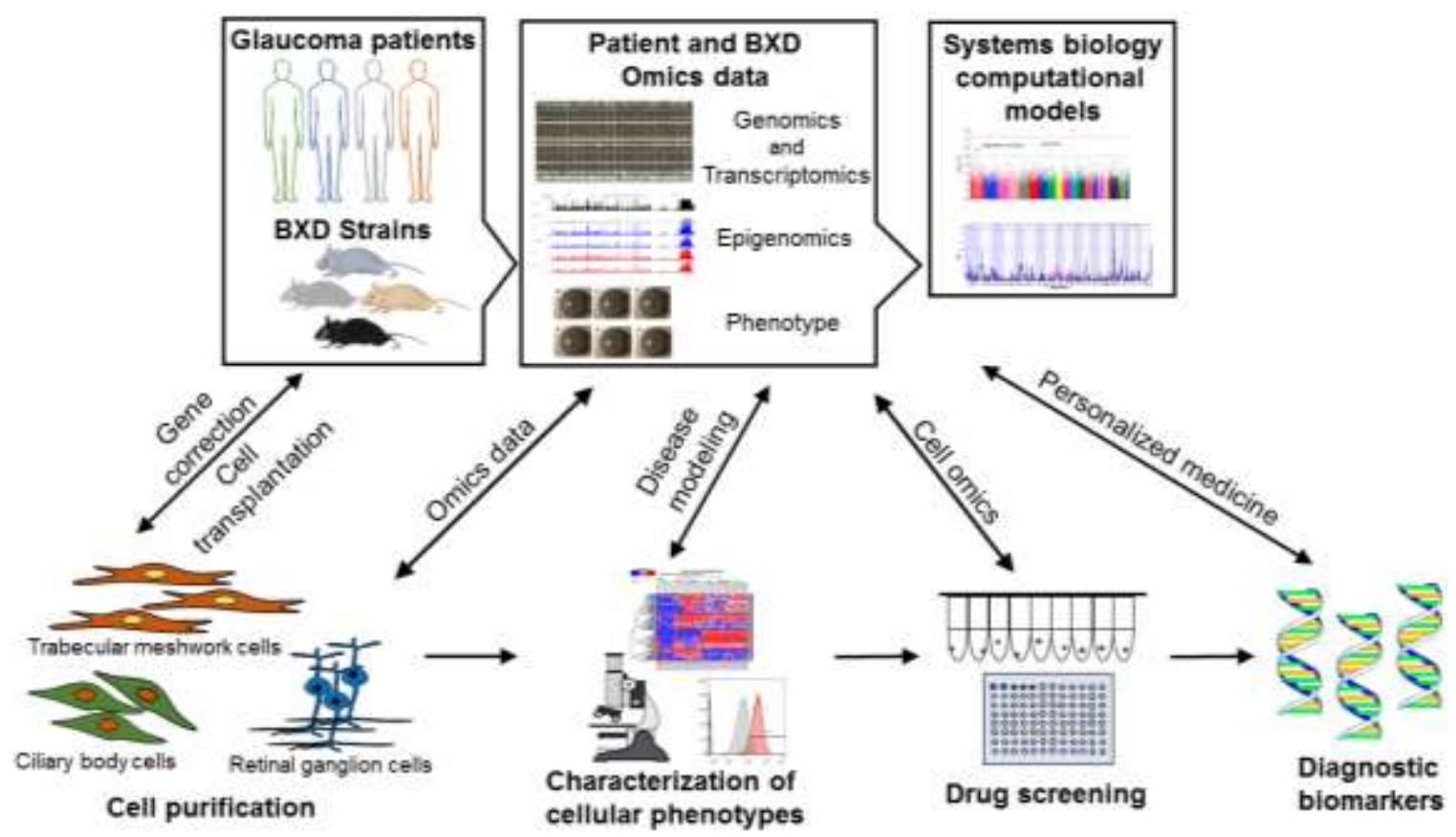

Figure 5-1. Glaucomics

The integrated use of multi-omics and systems science approaches towards rational discovery, development, and tandem applications of diagnostics and therapeutics, for glaucoma specifically, and for personalized visual health. 


\section{LIST OF REFERENCES}

1. Weinreb RN, Aung T, Medeiros FA. The pathophysiology and treatment of glaucoma: a review. Jama. 2014;311(18):1901-11.

2. Casson RJ, Chidlow G, Wood JPM, Crowston JG, Goldberg I. Definition of glaucoma: clinical and experimental concepts. Clinical \& experimental ophthalmology. 2012;40(4):341-9.

3. Quigley HA. New paradigms in the mechanisms and management of glaucoma. Eye. 2004;19(12):1241-8.

4. De Voogd S, Ikram MK, Wolfs RCW, Jansonius NM, Hofman A, De Jong PTVM. Incidence of open-angle glaucoma in a general elderly population: The Rotterdam Study. Ophthalmology. 2005;112(9):1487-93.

5. Ntim-Amponsah CT, Amoaku WMK, Ofosu-Amaah S, Ewusi RK, IdirisuriyaKhair R, Nyatepe-Coo E, et al. Prevalence of glaucoma in an African population. Eye. 2004;18(5):491-7.

6. Rotchford AP, Kirwan JF, Muller MA, Johnson GJ, Roux P. Temba glaucoma study: A population-based cross-sectional survey in urban South Africa. Ophthalmology. 2003;110(2):376-82.

7. Omoti AE, Edema OT. A review of the risk factors in primary open angle glaucoma. Nigerian journal of clinical practice. 2007;10(1):79-82.

8. Buus DR, Anderson DR. Peripapillary crescents and halos in normal-tension glaucoma and ocular hypertension. Ophthalmology. 1989;96(1):16-9.

9. Weinreb RN, Tee Khaw P. Primary open-angle glaucoma. Lancet. 2004;363(9422):1711-20.

10. Tielsch JM, Katz J, Singh K, Quigley HA, Gottsch JD, Javitt J, et al. A population-based evaluation of glaucoma screening: the Baltimore Eye Survey. American journal of epidemiology. 1991;134(10):1102-10.

11. Klein BE, Klein R, Sponsel WE, Franke T, Cantor LB, Martone J, et al. Prevalence of glaucoma. The Beaver Dam Eye Study. Ophthalmology. 1992;99(10):1499-504.

12. Gupta N, Weinreb RN. New definitions of glaucoma. Current opinion in ophthalmology. 1997;8(2):38-41.

13. Hendrickx KH, van den Enden A, Rasker MT, Hoyng PF. Cumulative incidence of patients with disc hemorrhages in glaucoma and the effect of therapy. Ophthalmology. 1994;101(7):1165-72.

14. Kwon YH, Fingert JH, Kuehn MH, Alward WLM. Primary Open-Angle Glaucoma. New England Journal of Medicine. 2009;360(11):1113-24.

15. Carbonaro F, Andrew T, Mackey DA, Spector TD, Hammond CJ. Heritability of intraocular pressure: a classical twin study. The British journal of ophthalmology. 2008;92(8):1125-8.

16. van Koolwijk LME, Despriet DDG, van Duijn CM, Pardo Cortes LM, Vingerling JR, Aulchenko YS, et al. Genetic Contributions to Glaucoma: Heritability of Intraocular Pressure, Retinal Nerve Fiber Layer Thickness, and Optic Disc Morphology. Investigative ophthalmology \& visual science. 2007;48(8):3669-76. 
17. van Koolwijk LME, Ramdas WD, Ikram MK, Jansonius NM, Pasutto F, Hysi PG, et al. Common Genetic Determinants of Intraocular Pressure and Primary OpenAngle Glaucoma. PLoS genetics. 2012;8(5):e1002611.

18. Monemi S, Spaeth G, DaSilva A, Popinchalk S, Ilitchev E, Liebmann J, et al. Identification of a novel adult-onset primary open-angle glaucoma (POAG) gene on 5q22.1. Human molecular genetics. 2005;14(6):725-33.

19. Hauser MA, Allingham RR, Linkroum K, Wang J, LaRocque-Abramson K, Figueiredo D, et al. Distribution of WDR36 DNA sequence variants in patients with primary open-angle glaucoma. Investigative ophthalmology \& visual science. 2006;47(6):2542-6.

20. van Koolwijk LM, Ramdas WD, Ikram MK, Jansonius NM, Pasutto F, Hysi PG, et al. Common genetic determinants of intraocular pressure and primary openangle glaucoma. PLoS Genet. 2012;8(5):e1002611.

21. Heijl A, Leske MC, Bengtsson B, Hyman L, Bengtsson B, Hussein M, et al. Reduction of intraocular pressure and glaucoma progression - Results from the early manifest glaucoma trial. Archives of ophthalmology. 2002;120(10):1268-79.

22. Kass MA, Heuer DK, Higginbotham EJ, Johnson CA, Keltner JL, Miller JP, et al. The Ocular Hypertension Treatment Study - A randomized trial determines that topical ocular hypotensive medication delays or prevents the onset of primary open-angle glaucoma. Archives of ophthalmology. 2002;120(6):701-13.

23. Alward WLM. Medical Management of Glaucoma. New England Journal of Medicine. 1998;339(18):1298-307.

24. Weinreb RN, Khaw PT. Primary open-angle glaucoma. The Lancet. 2004;363(9422):1711-20.

25. Akhtar RS, Ness JM, Roth KA. Bcl-2 family regulation of neuronal development and neurodegeneration. Biochimica et Biophysica Acta (BBA) - Molecular Cell Research. 2004;1644(2-3):189-203.

26. Bull ND, Guidi A, Goedert M, Martin KR, Spillantini MG. Reduced Axonal Transport and Increased Excitotoxic Retinal Ganglion Cell Degeneration in Mice Transgenic for Human Mutant P301S Tau. PloS one. 2012;7(4):e34724.

27. Bull ND, Chidlow G, Wood JP, Martin KR, Casson RJ. The mechanism of axonal degeneration after perikaryal excitotoxic injury to the retina. Experimental neurology. 2012;236(1):34-45.

28. Li Y, Schlamp CL, Nickells RW. Experimental induction of retinal ganglion cell death in adult mice. Investigative ophthalmology \& visual science. 1999;40(5):1004-8.

29. Schuettauf F, Stein T, Choragiewicz TJ, Rejdak R, Bolz S, Zurakowski D, et al. Caspase inhibitors protect against NMDA-mediated retinal ganglion cell death. Clinical \& experimental ophthalmology. 2011;39(6):545-54.

30. Berkelaar M, Clarke DB, Wang YC, Bray GM, Aguayo AJ. Axotomy results in delayed death and apoptosis of retinal ganglion cells in adult rats. Journal of Neuroscience. 1994;14(7):4368-74.

31. Garcia-Valenzuela E, Shareef S, Walsh J, Sharma SC. Programmed cell death of retinal ganglion cells during experimental glaucoma. Experimental eye research. 1995;61(1):33-44. 
32. Wakabayashi T, Kosaka J, Oshika T. JNK inhibitory kinase is up-regulated in retinal ganglion cells after axotomy and enhances BimEL expression level in neuronal cells. Journal of neurochemistry. 2005;95(2):526-36.

33. Nickells RW, Semaan SJ, Schlamp CL. Involvement of the Bcl2 gene family in the signaling and control of retinal ganglion cell death. Progress in brain research. 2008;173:423-35.

34. Babizhayev MA, Bunin Ya A. Lipid peroxidation in open-angle glaucoma. Acta Ophthalmologica. 1989;67(4):371-7.

35. Izzotti A, Saccà SC, Cartiglia C, De Flora S. Oxidative deoxyribonucleic acid damage in the eyes of glaucoma patients. American Journal of Medicine. 2003;114(8):638-46.

36. Ko ML, Peng PH, Ma MC, Ritch R, Chen CF. Dynamic changes in reactive oxygen species and antioxidant levels in retinas in experimental glaucoma. Free Radical Biology and Medicine. 2005;39(3):365-73.

37. Ahmed F, Brown KM, Stephan DA, Morrison JC, Johnson EC, Tomarev SI. Microarray analysis of changes in mRNA levels in the rat retina after experimental elevation of intraocular pressure. Investigative Ophthalmology and Visual Science. 2004;45(4):1247-58.

38. Howell GR, Macalinao DG, Sousa GL, Walden M, Soto I, Kneeland SC, et al. Molecular clustering identifies complement and endothelin induction as early events in a mouse model of glaucoma. The Journal of clinical investigation. 2011;121(4):1429-44.

39. Kuehn MH, Kim CY, Ostojic J, Bellin M, Alward WLM, Stone EM, et al. Retinal synthesis and deposition of complement components induced by ocular hypertension. Experimental eye research. 2006;83(3):620-8.

40. Levin LA, Danesh-Meyer HV. Lost in translation: bumps in the road between bench and bedside. Jama. 2010;303(15):1533-4.

41. Fingert JH. Primary open-angle glaucoma genes. Eye (London, England). 2011;25(5):587-95.

42. Sheffield VC, Stone EM, Alward WLM, Drack AV, Johnson AT, Streb LM, et al. Genetic linkage of familial open angle glaucoma to chromosome 1q21-q31. Nature genetics. 1993;4(1):47-50.

43. Sarfarazi M, Child A, Stoilova D, Brice G, Desai T, Trifan OC, et al. Localization of the fourth locus (GLC1E) for adult-onset primary open- angle glaucoma to the 10p15-p14 region. American journal of human genetics. 1998;62(3):641-52.

44. Ramdas WD, van Koolwijk LM, Ikram MK, Jansonius NM, de Jong PT, Bergen AA, et al. A genome-wide association study of optic disc parameters. PLoS genetics. 2010;6(6).

45. Thorleifsson G, Walters GB, Hewitt AW, Masson G, Helgason A, Dewan A, et al. Common variants near CAV1 and CAV2 are associated with primary open-angle glaucoma. Nature genetics. 2010;42(10):906-9.

46. Hirschhorn JN, Daly MJ. Genome-wide association studies for common diseases and complex traits. Nature Reviews Genetics. 2005;6(2):95-108.

47. Bush WS, Moore JH. Chapter 11: Genome-Wide Association Studies. PLoS Computational Biology. 2012;8(12):e1002822. 
48. Meguro A, Inoko H, Ota M, Mizuki N, Bahram S. Genome-wide association study of normal tension glaucoma: common variants in SRBD1 and ELOVL5 contribute to disease susceptibility. Ophthalmology. 2010;117(7):1331-8.e5.

49. Li Z, Allingham RR, Nakano M, Jia L, Chen Y, Ikeda Y, et al. A common variant near TGFBR3 is associated with primary open angle glaucoma. Human molecular genetics. 2015.

50. Wiggs JL, Yaspan BL, Hauser MA, Kang JH, Allingham RR, Olson LM, et al. Common variants at 9p21 and 8q22 are associated with increased susceptibility to optic nerve degeneration in glaucoma. PLoS Genet. 2012;8(4):e1002654.

51. Osman W, Low SK, Takahashi A, Kubo M, Nakamura Y. A genome-wide association study in the Japanese population confirms 9p21 and 14q23 as susceptibility loci for primary open angle glaucoma. Human molecular genetics. 2012;21(12):2836-42.

52. Gallardo ME, Lopez-Rios J, Fernaud-Espinosa I, Granadino B, Sanz R, Ramos C, et al. Genomic cloning and characterization of the human homeobox gene SIX6 reveals a cluster of SIX genes in chromosome 14 and associates SIX6 hemizygosity with bilateral anophthalmia and pituitary anomalies. Genomics. 1999;61(1):82-91.

53. Civelek M, Lusis AJ. Systems genetics approaches to understand complex traits. Nat Rev Genet. 2014;15(1):34-48.

54. van der Sijde MR, Ng A, Fu J. Systems genetics: From GWAS to disease pathways. Biochimica et Biophysica Acta (BBA) - Molecular Basis of Disease. 2014;1842(10):1903-9.

55. Bailey DW. Recombinant-inbred strains. An aid to finding identity, linkage, and function of histocompatibility and other genes. Transplantation. 1971;11(3):3257.

56. .L.M S. Mouse Genetics. New York: Oxford University Press; 1995.

57. A TB. Recombinant inbred strains. G LMLaSA, editor. Oxford: Oxford Universsity Press; 1989. 773-96 p.

58. Peirce JL, Chesler EJ, Williams RW, Lu L. Genetic architecture of the mouse hippocampus: identification of gene loci with selective regional effects. Genes, brain, and behavior. 2003;2(4):238-52.

59. Swaminathan S, Lu H, Williams RW, Lu L, Jablonski MM. Genetic modulation of the iris transillumination defect: a systems genetics analysis using the expanded family of BXD glaucoma strains. Pigment cell \& melanoma research. 2013.

60. Chintalapudi SM-T, Vanessa; Williams, Robert; Jablonski, Monica. Multipronged Approach to Identify and Validate a Novel Upstream Regulator of Sncg in Mouse Retinal Ganglion Cells (under review). The FEBS Journal. 2015.

61. Taylor BA. Recombinant inbred strains. H M, editor. New York: Academic; 1978. 423-38 p.

62. Klein TW. Analysis of major gene effects using recombinant inbred strains and related congenic lines. Behavior genetics. 1978;8(3):261-8.

63. Lander ES, Botstein D. Mapping mendelian factors underlying quantitative traits using RFLP linkage maps. Genetics. 1989;121(1):185-99.

64. John SW, Anderson MG, Smith RS. Mouse genetics: a tool to help unlock the mechanisms of glaucoma. Journal of glaucoma. 1999;8(6):400-12. 
65. Schlamp CL, Li Y, Dietz JA, Janssen KT, Nickells RW. Progressive ganglion cell loss and optic nerve degeneration in $\mathrm{DBA} / 2 \mathrm{~J}$ mice is variable and asymmetric. BMC neuroscience. 2006; 7:66.

66. Anderson MG, Smith RS, Hawes NL, Zabaleta A, Chang B, Wiggs JL, et al. Mutations in genes encoding melanosomal proteins cause pigmentary glaucoma in DBA/2J mice. Nature genetics. 2002;30(1):81-5.

67. Lynch S, Yanagi G, DelBono E, Wiggs JL. DNA sequence variants in the tyrosinase-related protein 1 (TYRP1) gene are not associated with human pigmentary glaucoma. Molecular vision. 2002;8:127-9.

68. Peirce JL, Lu L, Gu J, Silver LM, Williams RW. A new set of BXD recombinant inbred lines from advanced intercross populations in mice. BMC genetics. 2004;5:7.

69. Lu H, Wang X, Pullen M, Guan H, Chen H, Sahu S, et al. Genetic dissection of the Gpnmb network in the eye. Investigative ophthalmology \& visual science. 2011;52(7):4132-42.

70. Jablonski MM, Freeman NE, Orr WE, Templeton JP, Lu L, Williams RW, et al. Genetic pathways regulating glutamate levels in retinal Muller cells.

Neurochemical research. 2011;36(4):594-603.

71. Geisert EE, Lu L, Freeman-Anderson NE, Templeton JP, Nassr M, Wang X, et al. Gene expression in the mouse eye: an online resource for genetics using 103 strains of mice. Molecular vision. 2009;15:1730-63.

72. Peirce JL, Li H, Wang J, Manly KF, Hitzemann RJ, Belknap JK, et al. How replicable are mRNA expression QTL? Mammalian genome : official journal of the International Mammalian Genome Society. 2006;17(6):643-56.

73. Bystrykh L, Weersing E, Dontje B, Sutton S, Pletcher MT, Wiltshire T, et al. Uncovering regulatory pathways that affect hematopoietic stem cell function using 'genetical genomics'. Nature genetics. 2005;37(3):225-32.

74. Miyairi I, Tatireddigari VR, Mahdi OS, Rose LA, Belland RJ, Lu L, et al. The p47 GTPases Iigp2 and Irgb10 regulate innate immunity and inflammation to murine Chlamydia psittaci infection. Journal of immunology. 2007;179(3):181424.

75. Chesler EJ, Lu L, Shou S, Qu Y, Gu J, Wang J, et al. Complex trait analysis of gene expression uncovers polygenic and pleiotropic networks that modulate nervous system function. Nature genetics. 2005;37(3):233-42.

76. Gaglani SM, Lu L, Williams RW, Rosen GD. The genetic control of neocortex volume and covariation with neocortical gene expression in mice. BMC neuroscience. 2009;10:44.

77. Doerge RW. Mapping and analysis of quantitative trait loci in experimental populations. Nat Rev Genet. 2002;3(1):43-52.

78. Grisel JE. Quantitative trait locus analysis. Alcohol research \& health : the journal of the National Institute on Alcohol Abuse and Alcoholism. 2000;24(3):169-74.

79. Vignal A, Milan D, SanCristobal M, Eggen A. A review on SNP and other types of molecular markers and their use in animal genetics. Genetics, selection, evolution : GSE. 2002;34(3):275-305.

80. Broman KW, Sen S. A Guide to QTL Mapping with R/qtl: Springer; 2009. 
81. Peters LL, Robledo RF, Bult CJ, Churchill GA, Paigen BJ, Svenson KL. The mouse as a model for human biology: a resource guide for complex trait analysis. Nat Rev Genet. 2007;8(1):58-69.

82. Lu H, Li L, Watson ER, Williams RW, Geisert EE, Jablonski MM, et al. Complex interactions of Tyrp1 in the eye. Molecular vision. 2011;17:2455-68.

83. Grundberg E, Adoue V, Kwan T, Ge B, Duan QL, Lam KC, et al. Global analysis of the impact of environmental perturbation on cis-regulation of gene expression. PLoS Genet. 2011;7(1):e1001279.

84. Majewski J, Pastinen T. The study of eQTL variations by RNA-seq: from SNPs to phenotypes. Trends in genetics : TIG. 2011;27(2):72-9.

85. Stranger BE, Montgomery SB, Dimas AS, Parts L, Stegle O, Ingle CE, et al. Patterns of cis regulatory variation in diverse human populations. PLoS Genet. 2012;8(4):e1002639.

86. van Nas A, Ingram-Drake L, Sinsheimer JS, Wang SS, Schadt EE, Drake T, et al. Expression quantitative trait loci: replication, tissue- and sex-specificity in mice. Genetics. 2010;185(3):1059-68.

87. Petretto E, Mangion J, Dickens NJ, Cook SA, Kumaran MK, Lu H, et al. Heritability and tissue specificity of expression quantitative trait loci. PLoS Genet. 2006;2(10):e172.

88. P HR. Handbook of fluorescent probes and research chemicals. Eugene1996.

89. Donnenberg VS, Ulrich H, Tárnok A. Cytometry in Stem Cell Research and Therapy. Cytometry Part A : the journal of the International Society for Analytical Cytology. 2013;83(1):1-4.

90. Drouet M, Lees O. Clinical applications of flow cytometry in hematology and immunology. Biology of the cell / under the auspices of the European Cell Biology Organization. 1993;78(1-2):73-8.

91. Orfao A, Ciudad J, Gonzalez M, Lopez A, del Mar Abad M, Paz Bouza JI, et al. Flow cytometry in the diagnosis of cancer. Scandinavian journal of clinical and laboratory investigation Supplementum. 1995;221:145-52.

92. Kuszynski C. Flow Cytometry in Neuroscience Research. In: Xiong H, Gendelman EH, editors. Current Laboratory Methods in Neuroscience Research. New York, NY: Springer New York; 2014. p. 177-84.

93. Davis JL, Viciana AL, Ruiz P. Diagnosis of Intraocular Lymphoma by Flow Cytometry. American journal of ophthalmology.124(3):362-72.

94. Cousins SW, Streilein JW. Flow cytometric detection of lymphocyte proliferation in eyes with immunogenic inflammation. Investigative ophthalmology \& visual science. 1990;31(10):2111-22.

95. Davis JL. Intraocular lymphoma: a clinical perspective. Eye. 2013;27(2):153-62.

96. Mrugacz M, Zak J, Bakunowicz-Lazarczyk A, Wysocka J, Minarowska A. Flow cytometric analysis of HLA-DR antigen in conjunctival epithelial cells of patients with cystic fibrosis. Eye. 2006;21(8):1062-6.

97. Chen Y, Chauhan SK, Soo Lee H, Saban DR, Dana R. Chronic dry eye disease is principally mediated by effector memory Th17 cells. Mucosal Immunol. 2014;7(1):38-45.

98. Baraniskin A, Kuhnhenn J, Schlegel U, Chan A, Deckert M, Gold R, et al. Identification of microRNAs in the cerebrospinal fluid as marker for primary 
diffuse large B-cell lymphoma of the central nervous system. Blood. 2011;117(11):3140-6.

99. Davis JL, Viciana AL, Ruiz P. Diagnosis of intraocular lymphoma by flow cytometry. American journal of ophthalmology. 1997;124(3):362-72.

100. Felberg NT, Augsburger JJ, Shields JA, Goldschmidt J, Pronesti G, Haimowitz A. Antigenic modulation in retinoblastoma: a flow cytometric study. Investigative ophthalmology \& visual science. 1985;26(9):1306-9.

101. Detrick B, Evans CH, Chader G, Percopo CM, Hooks JJ. Cytokine-induced modulation of cellular proteins in retinoblastoma. Analysis by flow cytometry. Investigative ophthalmology \& visual science. 1991;32(6):1714-22.

102. Balla MM, Vemuganti GK, Kannabiran C, Honavar SG, Murthy R. Phenotypic characterization of retinoblastoma for the presence of putative cancer stem-like cell markers by flow cytometry. Investigative ophthalmology \& visual science. 2009;50(4):1506-14.

103. Samuel MA, Zhang Y, Meister M, Sanes JR. Age-related alterations in neurons of the mouse retina. The Journal of neuroscience : the official journal of the Society for Neuroscience. 2011;31(44):16033-44.

104. Portillo J-AC, Okenka G, Kern TS, Subauste CS. Identification of primary retinal cells and ex vivo detection of proinflammatory molecules using flow cytometry. Molecular vision. 2009;15:1383-9.

105. Chang Z-Y, Lu D-W, Yeh M-K, Chiang C-H. A Novel High-Content Flow Cytometric Method for Assessing the Viability and Damage of Rat Retinal Ganglion Cells. PloS one. 2012;7(3):e33983.

106. Kennedy CJ, Rakoczy PE, Constable IJ. A simple flow cytometric technique to quantify rod outer segment phagocytosis in cultured retinal pigment epithelial cells. Current eye research. 1996;15(9):998-1003.

107. Srivastava GK, Reinoso R, Singh AK, Fernandez-Bueno I, Martino M, GarciaGutierrez MT, et al. Flow cytometry assessment of the purity of human retinal pigment epithelial primary cell cultures. Journal of immunological methods. 2013;389(1-2):61-8.

108. Xu XL, Singh HP, Wang L, Qi D-L, Poulos BK, Abramson DH, et al. Rb suppresses human cone-precursor-derived retinoblastoma tumours. Nature. 2014;514(7522):385-8.

109. Feodorova Y, Koch M, Bultman S, Michalakis S, Solovei I. Quick and reliable method for retina dissociation and separation of rod photoreceptor perikarya from adult mice. MethodsX. 2015;2:39-46.

110. Lakowski J, Gonzalez-Cordero A, West EL, Han Y-T, Welby E, Naeem A, et al. Transplantation of Photoreceptor Precursors Isolated via a Cell Surface Biomarker Panel From Embryonic Stem Cell-Derived Self-Forming Retina. STEM CELLS. 2015;33(8):2469-82.

111. Liu CJ, Chou CK, Chen SJ, Yeh TS, Chiu AW, Liu JH. Flow cytometric study of anterior chamber aqueous humor after neodymium: yttrium aluminum garnet laser iridotomy. Annals of ophthalmology. 1993;25(5):174-9.

112. Cvenkel B, Kopitar AN, \#353, Ihan A. Inflammatory Molecules in Aqueous Humour and on Ocular Surface and Glaucoma Surgery Outcome. Mediators of Inflammation. 2010;2010. 
113. McElnea EM, Quill B, Docherty NG, Irnaten M, Siah WF, Clark AF, et al. Oxidative stress, mitochondrial dysfunction and calcium overload in human lamina cribrosa cells from glaucoma donors. Molecular vision. 2011;17:1182-91.

114. Nair KS, Barbay J, Smith RS, Masli S, John SWM. Determining immune components necessary for progression of pigment dispersing disease to glaucoma in DBA/2J mice. BMC genetics. 2014;15:42-.

115. Pease ME, Cone FE, Gelman S, Son JL, Quigley HA. Calibration of the TonoLab tonometer in mice with spontaneous or experimental glaucoma. Investigative ophthalmology \& visual science. 2011;52(2):858-64.

116. Wiggs JL, Hauser MA, Abdrabou W, Allingham RR, Budenz DL, DelBono E, et al. The NEIGHBOR Consortium Primary Open Angle Glaucoma Genome-wide Association Study: Rationale, Study design and Clinical variables. Journal of glaucoma. 2013;22(7):517-25.

117. Freeman NE, Templeton JP, Orr WE, Lu L, Williams RW, Geisert EE. Genetic networks in the mouse retina: growth associated protein 43 and phosphatase tensin homolog network. Molecular vision. 2011;17:1355-72.

118. Williams RW, Gu J, Qi S, Lu L. The genetic structure of recombinant inbred mice: high-resolution consensus maps for complex trait analysis. Genome biology. 2001;2(11):RESEARCH0046.

119. Lu H, Lu L, Williams RW, Jablonski MM. Iris transillumination defect and its gene modulators do not correlate with intraocular pressure in the BXD family of mice. Molecular vision. 2016;22:224-33.

120. Mulligan MK, Wang X, Adler AL, Mozhui K, Lu L, Williams RW. Complex control of GABA(A) receptor subunit mRNA expression: variation, covariation, and genetic regulation. PloS one. 2012;7(4):e34586.

121. Klugbauer N, Lacinová L, Marais E, Hobom M, Hofmann F. Molecular Diversity of the Calcium Channel $\alpha 2 \delta$ Subunit. The Journal of Neuroscience. 1999;19(2):684-91.

122. Dolphin AC. The $\alpha 2 \delta$ subunits of voltage-gated calcium channels. Biochimica et Biophysica Acta (BBA) - Biomembranes. 2013;1828(7):1541-9.

123. Chandra A, Mitry D, Wright A, Campbell H, Charteris DG. Genome-wide association studies: applications and insights gained in Ophthalmology. Eye. 2014;28(9):1066-79.

124. He Y, Ge J, Tombran-Tink J. Mitochondrial defects and dysfunction in calcium regulation in glaucomatous trabecular meshwork cells. Invest Ophthalmol Vis Sci. 2008;49(11):4912-22.

125. Andersen JS, Pralea AM, DelBono EA, Haines JL, Gorin MB, Schuman JS, et al. A gene responsible for the pigment dispersion syndrome maps to chromosome 7q35-q36. Archives of ophthalmology. 1997;115(3):384-8.

126. Aung T, Ozaki M, Mizoguchi T, Allingham RR, Li Z, Haripriya A, et al. A common variant mapping to CACNA1A is associated with susceptibility to exfoliation syndrome. Nature genetics. 2015;47(4):387-92.

127. Huang W, Fileta JB, Dobberfuhl A, Filippopolous T, Guo Y, Kwon G, et al. Calcineurin cleavage is triggered by elevated intraocular pressure, and calcineurin inhibition blocks retinal ganglion cell death in experimental glaucoma. 
Proceedings of the National Academy of Sciences of the United States of America. 2005;102(34):12242-7.

128. Wang Y, White MG, Akay C, Chodroff RA, Robinson J, Lindl KA, et al. Activation of cyclin-dependent kinase 5 by calpains contributes to human immunodeficiency virus-induced neurotoxicity. Journal of neurochemistry. 2007;103(2):439-55.

129. Cervia D, Casini G, Bagnoli P. Physiology and pathology of somatostatin in the mammalian retina: a current view. Molecular and cellular endocrinology. 2008;286(1-2):112-22.

130. Hartwick AT, Hamilton CM, Baldridge WH. Glutamatergic calcium dynamics and deregulation of rat retinal ganglion cells. The Journal of physiology. 2008;586(14):3425-46.

131. Ryskamp DA, Witkovsky P, Barabas P, Huang W, Koehler C, Akimov NP, et al. The polymodal ion channel transient receptor potential vanilloid 4 modulates calcium flux, spiking rate, and apoptosis of mouse retinal ganglion cells. The Journal of neuroscience : the official journal of the Society for Neuroscience. 2011;31(19):7089-101.

132. Kong GY, Van Bergen NJ, Trounce IA, Crowston JG. Mitochondrial dysfunction and glaucoma. Journal of glaucoma. 2009;18(2):93-100.

133. Kawasaki A, Otori Y, Barnstable CJ. Muller cell protection of rat retinal ganglion cells from glutamate and nitric oxide neurotoxicity. Investigative ophthalmology \& visual science. 2000;41(11):3444-50.

134. Araie M, Mayama C. Use of calcium channel blockers for glaucoma. Progress in retinal and eye research. 2011;30(1):54-71.

135. Lee DA, Higginbotham EJ. Glaucoma and its treatment: A review. American Journal of Health-System Pharmacy. 2005;62(7):691-9.

136. Atlante A, Calissano P, Bobba A, Giannattasio S, Marra E, Passarella S. Glutamate neurotoxicity, oxidative stress and mitochondria. FEBS letters. 2001;497(1):1-5.

137. Inokuchi Y, Nakajima Y, Shimazawa M, Kurita T, Kubo M, Saito A, et al. Effect of an inducer of $\mathrm{BiP}$, a molecular chaperone, on endoplasmic reticulum (ER) stress-induced retinal cell death. Investigative ophthalmology \& visual science. 2009;50(1):334-44.

138. Doh SH, Kim JH, Lee KM, Park HY, Park CK. Retinal ganglion cell death induced by endoplasmic reticulum stress in a chronic glaucoma model. Brain research. 2010;1308:158-66.

139. Surguchov A, McMahan B, Masliah E, Surgucheva I. Synucleins in ocular tissues. Journal of neuroscience research. 2001;65(1):68-77.

140. Kruger R, Schols L, Muller T, Kuhn W, Woitalla D, Przuntek H, et al. Evaluation of the gamma-synuclein gene in German Parkinson's disease patients.

Neuroscience letters. 2001;310(2-3):191-3.

141. Rockenstein E, Hansen LA, Mallory M, Trojanowski JQ, Galasko D, Masliah E. Altered expression of the synuclein family mRNA in Lewy body and Alzheimer's disease. Brain research. 2001;914(1-2):48-56. 
142. Lavedan C, Leroy E, Dehejia A, Buchholtz S, Dutra A, Nussbaum RL, et al. Identification, localization and characterization of the human gamma-synuclein gene. Human genetics. 1998;103(1):106-12.

143. Soto I, Oglesby E, Buckingham BP, Son JL, Roberson ED, Steele MR, et al. Retinal ganglion cells downregulate gene expression and lose their axons within the optic nerve head in a mouse glaucoma model. The Journal of neuroscience : the official journal of the Society for Neuroscience. 2008;28(2):548-61.

144. Nookala S, Gandrakota R, Wohabrebbi A, Wang X, Howell D, Giorgianni F, et al. In search of the identity of the XAP-1 antigen: a protein localized to cone outer segments. Investigative ophthalmology \& visual science. 2010;51(5):2736-43.

145. Strober W. Trypan blue exclusion test of cell viability. Current protocols in immunology / edited by John E Coligan [et al]. 2001;Appendix 3:Appendix 3B.

146. Morales-Tirado V, Wichlan DG, Leimig TE, Street SE, Kasow KA, Riberdy JM. 1 alpha,25-dihydroxyvitamin D3 (vitamin D3) catalyzes suppressive activity on human natural regulatory $\mathrm{T}$ cells, uniquely modulates cell cycle progression, and augments FOXP3. Clinical immunology (Orlando, Fla). 2011;138(2):212-21.

147. Winzeler A, Wang JT. Purification and culture of retinal ganglion cells from rodents. Cold Spring Harb Protoc. 2013;2013(7):643-52.

148. Jeon CJ, Strettoi E, Masland RH. The major cell populations of the mouse retina. The Journal of neuroscience : the official journal of the Society for Neuroscience. 1998;18(21):8936-46.

149. Higuti T, Tsurumi C, Osaka F, Kawamura Y, Tsujita H, Yoshihara Y, et al. Molecular cloning of cDNA for the import precursor of human subunit $\mathrm{B}$ of $\mathrm{H}(+)$ ATP synthase in mitochondria. Biochemical and biophysical research communications. 1991;178(3):1014-20.

150. Matsuda C, Endo H, Ohta S, Kagawa Y. Gene structure of human mitochondrial ATP synthase gamma-subunit. Tissue specificity produced by alternative RNA splicing. The Journal of biological chemistry. 1993;268(33):24950-8.

151. Fujiwara T, Kawai A, Shimizu F, Hirano H, Okuno S, Takeda S, et al. Cloning, sequencing and expression of a novel cDNA encoding human vacuolar ATPase (14-kDa subunit). DNA research : an international journal for rapid publication of reports on genes and genomes. 1995;2(3):107-11.

152. Islam MM, Suzuki H, Yoneda M, Tanaka M. Primary structure of the smallest (6.4-kDa) subunit of human and bovine ubiquinol-cytochrome $\mathrm{c}$ reductase deduced from cDNA sequences. Biochemistry and molecular biology international. 1997;41(6):1109-16.

153. Valnot I, Kassis J, Chretien D, de Lonlay P, Parfait B, Munnich A, et al. A mitochondrial cytochrome $\mathrm{b}$ mutation but no mutations of nuclearly encoded subunits in ubiquinol cytochrome c reductase (complex III) deficiency. Human genetics. 1999;104(6):460-6.

154. Cavdar Koc E, Burkhart W, Blackburn K, Moseley A, Spremulli LL. The small subunit of the mammalian mitochondrial ribosome. Identification of the full complement of ribosomal proteins present. The Journal of biological chemistry. 2001;276(22):19363-74.

155. Kenmochi N, Suzuki T, Uechi T, Magoori M, Kuniba M, Higa S, et al. The human mitochondrial ribosomal protein genes: mapping of 54 genes to the 
chromosomes and implications for human disorders. Genomics. 2001;77(1-2):6570.

156. Papa S. The NDUFS4 nuclear gene of complex I of mitochondria and the cAMP cascade. Biochimica et biophysica acta. 2002;1555(1-3):147-53.

157. Leyva JA, Bianchet MA, Amzel LM. Understanding ATP synthesis: structure and mechanism of the F1-ATPase (Review). Molecular membrane biology. 2003;20(1):27-33.

158. Modena P, Testi MA, Facchinetti F, Mezzanzanica D, Radice MT, Pilotti S, et al. UQCRH gene encoding mitochondrial Hinge protein is interrupted by a translocation in a soft-tissue sarcoma and epigenetically inactivated in some cancer cell lines. Oncogene. 2003;22(29):4586-93.

159. Zhang Z, Gerstein M. Identification and characterization of over 100 mitochondrial ribosomal protein pseudogenes in the human genome. Genomics. 2003;81(5):468-80.

160. Mourtada-Maarabouni M, Kirkham L, Farzaneh F, Williams GT. Regulation of apoptosis by fau revealed by functional expression cloning and antisense expression. Oncogene. 2004;23(58):9419-26.

161. Hoefs SJ, Dieteren CE, Distelmaier F, Janssen RJ, Epplen A, Swarts HG, et al. NDUFA2 complex I mutation leads to Leigh disease. American journal of human genetics. 2008;82(6):1306-15.

162. Zanotto E, Lehtonen V, Jacobs HT. Modulation of Mrps 12/Sarsm promoter activity in response to mitochondrial stress. Biochimica et biophysica acta. 2008;1783(12):2352-62.

163. Vives-Bauza C, Magrane J, Andreu AL, Manfredi G. Novel role of ATPase subunit $\mathrm{C}$ targeting peptides beyond mitochondrial protein import. Molecular biology of the cell. 2010;21(1):131-9.

164. Colombi M, Molle KD, Benjamin D, Rattenbacher-Kiser K, Schaefer C, Betz C, et al. Genome-wide shRNA screen reveals increased mitochondrial dependence upon mTORC2 addiction. Oncogene. 2011;30(13):1551-65.

165. Hua T, Wu D, Ding W, Wang J, Shaw N, Liu ZJ. Studies of human 2,4-dienoyl CoA reductase shed new light on peroxisomal beta-oxidation of unsaturated fatty acids. The Journal of biological chemistry. 2012;287(34):28956-65.

166. Rooijers K, Loayza-Puch F, Nijtmans LG, Agami R. Ribosome profiling reveals features of normal and disease-associated mitochondrial translation. Nature communications. 2013;4:2886.

167. Pulliam DA, Deepa SS, Liu Y, Hill S, Lin AL, Bhattacharya A, et al. Complex IV-deficient Surf1(-/-) mice initiate mitochondrial stress responses. The Biochemical journal. 2014;462(2):359-71.

168. Rak M, Rustin P. Supernumerary subunits NDUFA3, NDUFA5 and NDUFA12 are required for the formation of the extramembrane arm of human mitochondrial complex I. FEBS letters. 2014;588(9):1832-8.

169. Abdulhag UN, Soiferman D, Schueler-Furman O, Miller C, Shaag A, Elpeleg O, et al. Mitochondrial complex IV deficiency, caused by mutated COX6B1, is associated with encephalomyopathy, hydrocephalus and cardiomyopathy. European journal of human genetics : EJHG. 2015;23(2):159-64. 
170. Petry F, McClive PJ, Botto M, Morley BJ, Morahan G, Loos M. The mouse C1q genes are clustered on chromosome 4 and show conservation of gene organization. Immunogenetics. 1996;43(6):370-6.

171. de la Fuente A, Bing N, Hoeschele I, Mendes P. Discovery of meaningful associations in genomic data using partial correlation coefficients. Bioinformatics (Oxford, England). 2004;20(18):3565-74.

172. Ma S, Gong Q, Bohnert HJ. An Arabidopsis gene network based on the graphical Gaussian model. Genome research. 2007;17(11):1614-25.

173. Fujita A, Sato JR, Garay-Malpartida HM, Yamaguchi R, Miyano S, Sogayar MC, et al. Modeling gene expression regulatory networks with the sparse vector autoregressive model. BMC systems biology. 2007;1:39.

174. Schafer J, Strimmer K. An empirical Bayes approach to inferring large-scale gene association networks. Bioinformatics (Oxford, England). 2005;21(6):754-64.

175. Surgucheva I, Shestopalov VI, Surguchov A. Effect of gamma-synuclein silencing on apoptotic pathways in retinal ganglion cells. The Journal of biological chemistry. 2008;283(52):36377-85.

176. Wilding C, Bell K, Beck S, Funke S, Pfeiffer N, Grus FH. gamma-Synuclein antibodies have neuroprotective potential on neuroretinal cells via proteins of the mitochondrial apoptosis pathway. PloS one. 2014;9(3):e90737.

177. Jiang Y, Liu YE, Goldberg ID, Shi YE. Gamma synuclein, a novel heat-shock protein-associated chaperone, stimulates ligand-dependent estrogen receptor alpha signaling and mammary tumorigenesis. Cancer research. 2004;64(13):4539-46.

178. Surgucheva I, Park BC, Yue BY, Tomarev S, Surguchov A. Interaction of myocilin with gamma-synuclein affects its secretion and aggregation. Cellular and molecular neurobiology. 2005;25(6):1009-33.

179. Vainberg IE, Lewis SA, Rommelaere H, Ampe C, Vandekerckhove J, Klein HL, et al. Prefoldin, a chaperone that delivers unfolded proteins to cytosolic chaperonin. Cell. 1998;93(5):863-73.

180. Takano M, Tashiro E, Kitamura A, Maita H, Iguchi-Ariga SM, Kinjo M, et al. Prefoldin prevents aggregation of alpha-synuclein. Brain research. 2014; 1542:186-94.

181. Abe A, Takahashi-Niki K, Takekoshi Y, Shimizu T, Kitaura H, Maita H, et al. Prefoldin Plays a Role as a Clearance Factor in Preventing Proteasome Inhibitorinduced Protein Aggregation. Journal of Biological Chemistry. 2013;288(39):27764-76.

182. Soergjerd KM, Zako T, Sakono M, Stirling PC, Leroux MR, Saito T, et al. Human Prefoldin Inhibits Amyloid-beta (A beta) Fibrillation and Contributes to Formation of Nontoxic A/beta Aggregates. Biochemistry. 2013;52(20):3532-42.

183. Lee Y, Smith RS, Jordan W, King BL, Won J, Valpuesta JM, et al. Prefoldin 5 Is Required for Normal Sensory and Neuronal Development in a Murine Model. Journal of Biological Chemistry. 2011;286(1):726-36.

184. Geissler S, Siegers K, Schiebel E. A novel protein complex promoting formation of functional $\alpha$ - and $\gamma$-tubulin1998 1998-02-15 00:00:00. 952-66 p.

185. Yu DY, Cringle SJ, Balaratnasingam C, Morgan WH, Yu PK, Su EN. Retinal ganglion cells: Energetics, compartmentation, axonal transport, cytoskeletons and vulnerability. Progress in retinal and eye research. 2013;36:217-46. 
186. Mozhui K, Ciobanu DC, Schikorski T, Wang X, Lu L, Williams RW. Dissection of a QTL hotspot on mouse distal chromosome 1 that modulates neurobehavioral phenotypes and gene expression. PLoS Genet. 2008;4(11):e1000260.

187. Ding Q, Cecarini V, Keller JN. Interplay between protein synthesis and degradation in the CNS: physiological and pathological implications. Trends in neurosciences. 2007;30(1):31-6.

188. Yalcin B, Willis-Owen SA, Fullerton J, Meesaq A, Deacon RM, Rawlins JN, et al. Genetic dissection of a behavioral quantitative trait locus shows that Rgs2 modulates anxiety in mice. Nature genetics. 2004;36(11):1197-202.

189. Gershenfeld HK, Neumann PE, Mathis C, Crawley JN, Li X, Paul SM. Mapping quantitative trait loci for open-field behavior in mice. Behavior genetics. 1997;27(3):201-10.

190. Caldarone B, Saavedra C, Tartaglia K, Wehner JM, Dudek BC, Flaherty L. Quantitative trait loci analysis affecting contextual conditioning in mice. Nature genetics. 1997;17(3):335-7.

191. Meyer-Franke A, Kaplan MR, Pfieger FW, Barres BA. Characterization of the signaling interactions that promote the survival and growth of developing retinal ganglion cells in culture. Neuron. 1995;15(4):805-19.

192. Ullian EM, Sapperstein SK, Christopherson KS, Barres BA. Control of Synapse Number by Glia. Science (New York, NY). 2001;291(5504):657-61.

193. Goldberg JL, Klassen MP, Hua Y, Barres BA. Amacrine-Signaled Loss of Intrinsic Axon Growth Ability by Retinal Ganglion Cells. Science (New York, NY). 2002;296(5574):1860-4.

194. Christopherson KS, Ullian EM, Stokes CCA, Mullowney CE, Hell JW, Agah A, et al. Thrombospondins Are Astrocyte-Secreted Proteins that Promote CNS Synaptogenesis. Cell. 2005;120(3):421-33.

195. Watkins TA, Emery B, Mulinyawe S, Barres BA. Distinct Stages of Myelination Regulated by $\gamma$-Secretase and Astrocytes in a Rapidly Myelinating CNS Coculture System. Neuron. 2008;60(4):555-69.

196. Quigley HA, Hohman RM, Addicks EM, Massof RW, Green WR. Morphologic changes in the lamina cribrosa correlated with neural loss in open-angle glaucoma. American journal of ophthalmology. 1983;95(5):673-91.

197. Hollenbeck PJ, Saxton WM. The axonal transport of mitochondria. Journal of cell science. 2005;118(Pt 23):5411-9.

198. Nakamura K. alpha-Synuclein and mitochondria: partners in crime? Neurotherapeutics : the journal of the American Society for Experimental NeuroTherapeutics. 2013;10(3):391-9.

199. Liu L, Xie R, Yang C, McKeehan WL. Dual function microtubule- and mitochondria-associated proteins mediate mitotic cell death. Cellular oncology : the official journal of the International Society for Cellular Oncology. 2009;31(5):393-405.

200. Kuznetsov AV, Javadov S, Guzun R, Grimm M, Saks V. Cytoskeleton and regulation of mitochondrial function: the role of beta-tubulin II. Frontiers in physiology. 2013;4:82.

201. Quigley HA. Number of people with glaucoma worldwide. The British journal of ophthalmology. 1996;80(5):389-93. 
202. Wood JP, Chidlow G, Tran T, Crowston JG, Casson RJ. A comparison of differentiation protocols for RGC-5 cells. Invest Ophthalmol Vis Sci. 2010;51(7):3774-83.

203. Van Bergen NJ, Wood JP, Chidlow G, Trounce IA, Casson RJ, Ju WK, et al. Recharacterization of the RGC-5 retinal ganglion cell line. Invest Ophthalmol Vis Sci. 2009;50(9):4267-72.

204. Dreher B, Sefton AJ, Ni SY, Nisbett G. The morphology, number, distribution and central projections of Class I retinal ganglion cells in albino and hooded rats. Brain, behavior and evolution. 1985;26(1):10-48.

205. Williams RW, Strom RC, Rice DS, Goldowitz D. Genetic and environmental control of variation in retinal ganglion cell number in mice. The Journal of neuroscience : the official journal of the Society for Neuroscience. 1996;16(22):7193-205.

206. Surgucheva I, Weisman AD, Goldberg JL, Shnyra A, Surguchov A. Gammasynuclein as a marker of retinal ganglion cells. Molecular vision. 2008;14:1540-8.

207. Kwong JM, Quan A, Kyung H, Piri N, Caprioli J. Quantitative analysis of retinal ganglion cell survival with Rbpms immunolabeling in animal models of optic neuropathies. Invest Ophthalmol Vis Sci. 2011;52(13):9694-702.

208. Nadal-Nicolas FM, Jimenez-Lopez M, Sobrado-Calvo P, Nieto-Lopez L, Canovas-Martinez I, Salinas-Navarro M, et al. Brn3a as a marker of retinal ganglion cells: qualitative and quantitative time course studies in naive and optic nerve-injured retinas. Invest Ophthalmol Vis Sci. 2009;50(8):3860-8.

209. Barres BA, Silverstein BE, Corey DP, Chun LL. Immunological, morphological, and electrophysiological variation among retinal ganglion cells purified by panning. Neuron. 1988;1(9):791-803.

210. Hong S, Iizuka Y, Kim CY, Seong GJ. Isolation of primary mouse retinal ganglion cells using immunopanning-magnetic separation. Molecular vision. 2012;18:2922-30.

211. Pennartz S PM, Pfrieger F. Purification of retinal ganglion cells from postnatal rats by magnetic cell sorting. MACSmore. 2010;12(2):16-8.

212. Jackson CJ, Garbett PK, Nissen B, Schrieber L. Binding of human endothelium to Ulex europaeus I-coated Dynabeads: application to the isolation of microvascular endothelium. Journal of cell science. 1990;96 ( Pt 2):257-62.

213. Sahagun G, Moore SA, Fabry Z, Schelper RL, Hart MN. Purification of murine endothelial cell cultures by flow cytometry using fluorescein-labeled griffonia simplicifolia agglutinin. The American journal of pathology. 1989;134(6):122732.

214. Julius MH, Masuda T, Herzenberg LA. Demonstration that antigen-binding cells are precursors of antibody-producing cells after purification with a fluorescenceactivated cell sorter. Proc Natl Acad Sci U S A. 1972;69(7):1934-8.

215. Jiang Y, Zhang Q, Steinle JJ. Beta-adrenergic receptor agonist decreases VEGF levels through altered eNOS and PKC signaling in diabetic retina. Growth factors (Chur, Switzerland). 2015;33(3):192-9.

216. Kasow KA, Morales-Tirado VM, Wichlan D, Shurtleff SA, Abraham A, Persons $\mathrm{DA}$, et al. Therapeutic in vivo selection of thymic-derived natural $\mathrm{T}$ regulatory 
cells following non-myeloablative hematopoietic stem cell transplant for IPEX. Clinical immunology (Orlando, Fla). 2011;141(2):169-76.

217. Morales-Tirado V, Sojka DK, Katzman SD, Lazarski CA, Finkelman FD, Urban JF, et al. Critical requirement for the Wiskott-Aldrich syndrome protein in Th2 effector function. Blood. 2010;115(17):3498-507.

218. Haeryfar SM, Hoskin DW. Thy-1: more than a mouse pan-T cell marker. Journal of immunology. 2004;173(6):3581-8.

219. Reif AE, Allen JM. THE AKR THYMIC ANTIGEN AND ITS DISTRIBUTION IN LEUKEMIAS AND NERVOUS TISSUES. The Journal of experimental medicine. 1964;120:413-33.

220. Watanabe M, Noguchi T, Tsukada Y. Regional, cellular, and subcellular distribution of Thy-1 antigen in rat nervous tissues. Neurochemical research. 1981;6(5):507-19.

221. Jakobs TC, Ben Y, Masland RH. CD15 immunoreactive amacrine cells in the mouse retina. The Journal of comparative neurology. 2003;465(3):361-71.

222. Uusitalo M, Schlotzer-Schrehardt U, Kivela T. Ultrastructural localization of the HNK-1 carbohydrate epitope to glial and neuronal cells of the human retina. Investigative ophthalmology \& visual science. 2003;44(3):961-4.

223. Kwong JM, Caprioli J, Piri N. RNA binding protein with multiple splicing: a new marker for retinal ganglion cells. Invest Ophthalmol Vis Sci. 2010;51(2):1052-8.

224. Unkeless JC, Kaplan G, Plutner H, Cohn ZA. Fc-receptor variants of a mouse macrophage cell line. Proc Natl Acad Sci U S A. 1979;76(3):1400-4.

225. Balogh P, Tew JG, Szakal AK. Simultaneous blockade of Fc gamma receptors and indirect labeling of mouse lymphocytes by the selective detection of allotyperestricted epitopes on the kappa chain of rat monoclonal antibodies. Cytometry. 2002;47(2):107-10.

226. Kornguth S, Auerbach R, Grieves J, Kahan L. Immunological reactivity of monoclonal antibodies prepared against large ganglion cells from bovine retina. Neuroscience letters. 1981;27(2):151-7.

227. Beale R, Beaton DW, Neuhoff V, Osborne NN. Enriched populations of rat retinal ganglion cells: Studies using a cell-type specific surface marker. Neurochem Int. 1983;5(6):691-6.

228. Sarthy PV, Curtis BM, Catterall WA. Retrograde labeling, enrichment, and characterization of retinal ganglion cells from the neonatal rat. The Journal of neuroscience : the official journal of the Society for Neuroscience. 1983;3(12):2532-44.

229. Shoge K, Mishima HK, Mukai S, Shinya M, Ishihara K, Kanno M, et al. Rat retinal ganglion cells culture enriched with the magnetic cell sorter. Neuroscience letters. 1999;259(2):111-4.

230. Templeton JP, Freeman NE, Nickerson JM, Jablonski MM, Rex TS, Williams $\mathrm{RW}$, et al. Innate immune network in the retina activated by optic nerve crush. Investigative ophthalmology \& visual science. 2013;54(4):2599-606.

231. A. Bouhenni R, Dunmire J, Sewell A, Edward DP. Animal Models of Glaucoma. Journal of Biomedicine and Biotechnology. 2012;2012:11.

232. Penrod NM, Cowper-Sal-lari R, Moore JH. Systems genetics for drug target discovery. Trends in pharmacological sciences. 2011;32(10):623-30. 
233. Chrysostomou V, Rezania F, Trounce IA, Crowston JG. Oxidative stress and mitochondrial dysfunction in glaucoma. Current opinion in pharmacology. 2013;13(1):12-5.

234. Leygraf A, Hohoff C, Freitag C, Willis-Owen SA, Krakowitzky P, Fritze J, et al. Rgs 2 gene polymorphisms as modulators of anxiety in humans? Journal of neural transmission (Vienna, Austria : 1996). 2006;113(12):1921-5.

235. Krishnamoorthy RR, Clark AF, Daudt D, Vishwanatha JK, Yorio T. A Forensic Path to RGC-5 Cell Line Identification: Lessons Learned. Invest Ophthalmol Vis Sci. 2013;54(8):5712-9.

236. Krishnamoorthy RR, Agarwal P, Prasanna G, Vopat K, Lambert W, Sheedlo HJ, et al. Characterization of a transformed rat retinal ganglion cell line. Brain research Molecular brain research. 2001;86(1-2):1-12.

237. The Editors Of Molecular V. On authentication of cell lines. Molecular vision. 2013;19:1848-51.

238. Hernandez M, Rodriguez FD, Sharma SC, Vecino E. Immunohistochemical changes in rat retinas at various time periods of elevated intraocular pressure. Molecular vision. 2009;15:2696-709.

239. Mead B, Thompson A, Scheven BA, Logan A, Berry M, Leadbeater W. Comparative evaluation of methods for estimating retinal ganglion cell loss in retinal sections and wholemounts. PloS one. 2014;9(10):e110612.

240. Frick KD, Kymes SM, Lee PP, Matchar DB, Pezzullo ML, Rein DB, et al. The Cost of Visual Impairment: Purposes, Perspectives, and Guidance. Investigative ophthalmology \& visual science. 2010;51(4):1801-5.

241. Gungor K, Hotez PJ, Ozdemir V, Aynacioglu S. Glaucomics: a call for systems diagnostics for 21(st) century ophthalmology and personalized visual health. Omics : a journal of integrative biology. 2014;18(5):275-9. 


\section{APPENDIX A. SUPPLEMENTAL DATA FOR CHAPTER 2}

Table A-1. IOP values of BXD strains at 9.1-13 months age

\begin{tabular}{ccccc}
\hline & $\begin{array}{c}\text { Average } \\
\text { Bemale }\end{array}$ & $\begin{array}{c}\text { Average } \\
\text { Male }\end{array}$ & $\begin{array}{c}\text { Overall } \\
\text { IOP }\end{array}$ & SE \\
Strains & IOP & IOP & 17.63 & 0.38 \\
\hline B6D2F1 & 18.25 & 17.00 & 17.63 & \\
C57BL/6J & 15.00 & 15.42 & 15.23 & 0.78 \\
DBA/2J & 16.77 & 17.00 & 16.84 & 0.70 \\
BXD1 & 18.20 & X & 18.20 & 1.08 \\
BXD2 & 11.33 & X & 11.33 & 0.83 \\
BXD5 & 12.17 & 14.17 & 13.17 & 1.98 \\
BXD6 & 12.50 & X & 12.50 & X \\
BXD8 & 17.00 & 17.00 & 17.00 & 2.11 \\
BXD9 & 11.75 & 16.75 & 14.25 & 1.61 \\
BXD11 & 14.83 & 14.60 & 14.75 & 0.94 \\
BXD12 & 13.25 & 15.50 & 14.38 & 1.48 \\
BXD13 & 13.50 & 13.25 & 13.38 & 0.38 \\
BXD14 & 18.75 & 20.00 & 19.38 & 0.43 \\
BXD15 & 15.67 & 17.00 & 16.33 & 0.54 \\
BXD16 & 15.88 & 18.50 & 16.75 & 0.87 \\
BXD18 & 19.25 & 17.00 & 18.13 & 0.83 \\
BXD19 & 13.08 & 15.25 & 14.17 & 0.86 \\
BXD20 & 10.67 & 11.33 & 11.00 & 1.08 \\
BXD24 & 18.00 & 16.00 & 17.00 & 1.00 \\
BXD27 & 19.64 & 16.60 & 18.38 & 1.13 \\
BXD29 & 13.50 & 15.83 & 14.67 & 1.11 \\
BXD31 & 14.33 & X & 12.63 & 1.73 \\
BXD32 & 13.33 & 14.75 & 13.90 & 0.70 \\
BXD33 & 19.00 & 17.00 & 18.00 & 0.56 \\
BXD34 & 18.57 & 19.63 & 18.95 & 0.79 \\
BXD36 & 14.88 & 14.75 & 14.83 & 0.46 \\
BXD38 & 9.25 & 10.25 & 9.58 & 0.93 \\
BXD39 & 11.50 & 14.50 & 13.21 & 1.14 \\
BXD40 & 14.50 & 12.25 & 13.75 & 0.64 \\
BXD42 & 9.50 & 12.88 & 11.75 & 0.88 \\
BXD43 & 17.63 & 18.13 & 17.88 & 0.47 \\
BXD45 & 17.25 & 15.00 & 16.50 & 1.52 \\
BXD48 & 9.25 & 9.33 & 11.90 & 0.95 \\
BXD0 & 12.33 & 12.75 & 12.50 & 2.13
\end{tabular}


Table A-1. (continued)

\begin{tabular}{ccccc}
\hline $\begin{array}{c}\text { BXD } \\
\text { Strains }\end{array}$ & $\begin{array}{c}\text { Average } \\
\text { Female IOP }\end{array}$ & $\begin{array}{c}\text { Average } \\
\text { Male } \\
\text { IOP }\end{array}$ & Overall IOP & SE \\
\hline BXD51 & 18.50 & 17.40 & 17.89 & 1.50 \\
BXD55 & 14.50 & 15.17 & 15.00 & 0.60 \\
BXD56 & 18.31 & 20.00 & 18.88 & 1.20 \\
BXD60 & 14.38 & 14.50 & 14.45 & 0.59 \\
BXD61 & 17.68 & 19.80 & 18.24 & 1.66 \\
BXD62 & 12.10 & 17.75 & 14.61 & 1.93 \\
BXD63 & X & 17.33 & 17.33 & 0.60 \\
BXD65 & 13.88 & 14.33 & 13.45 & 0.88 \\
BXD66 & 15.33 & 20.88 & 18.50 & 1.47 \\
BXD67 & 16.50 & 18.50 & 17.50 & 0.71 \\
BXD68 & 18.63 & 13.25 & 16.83 & 1.90 \\
BXD69 & 16.77 & 18.50 & 17.04 & 0.55 \\
BXD70 & 17.63 & 14.13 & 16.46 & 0.96 \\
BXD71 & 11.50 & 13.83 & 12.50 & 2.07 \\
BXD73 & 15.83 & 18.29 & 17.94 & 0.90 \\
BXD75 & 15.50 & 14.20 & 14.85 & 0.67 \\
BXD77 & 18.00 & 17.50 & 17.64 & 1.46 \\
BXD83 & 13.50 & 16.75 & 16.21 & 0.75 \\
BXD84 & 14.50 & 12.63 & 13.75 & 0.74 \\
BXD85 & 16.43 & 15.71 & 16.07 & 1.14 \\
BXD86 & 16.67 & X & 16.67 & 1.28 \\
BXD87 & 18.50 & 15.30 & 16.50 & 0.89 \\
BXD89 & 14.15 & 16.50 & 14.54 & 0.65 \\
BXD90 & 15.83 & 14.50 & 15.30 & 1.57 \\
BXD92 & 14.17 & 13.83 & 14.00 & 1.62 \\
BXD95 & 18.13 & 17.38 & 17.75 & 1.39 \\
BXD98 & 18.80 & 18.50 & 18.69 & 0.72 \\
BXD99 & 18.50 & 18.00 & 18.25 & 0.32 \\
BXD100 & 16.33 & 17.50 & 16.55 & 0.65 \\
BXD101 & 13.25 & X & 13.25 & 0.83 \\
BXD102 & 14.67 & 16.50 & 15.28 & 1.12 \\
\hline & & & &
\end{tabular}


Table A-2. Cacna2d1 expression in BXD strains whole eye

\begin{tabular}{lcl}
\multicolumn{1}{c}{ BXD } & \multicolumn{1}{c}{ Cacna2d1 } & \multicolumn{1}{c}{ SE } \\
Strains & Expession & \\
\hline B6D2F1 & 9.049 & 0.04 \\
D2B6F1 & 8.874 & 0.114 \\
C57BL/6J & 9.498 & 0.071 \\
DBA/2J & 7.543 & 0.148 \\
BXD1 & 7.927 & 0.355 \\
BXD2 & 7.813 & 0.338 \\
BXD5 & 7.646 & 0.102 \\
BXD6 & 7.759 & 0.058 \\
BXD8 & 8.883 & 0.15 \\
BXD9 & 7.502 & 0.602 \\
BXD11 & 7.314 & 0.42 \\
BXD12 & 7.833 & 0.117 \\
BXD13 & 8.847 & 0.4 \\
BXD14 & 8.897 & 0.625 \\
BXD15 & 8.827 & 0.397 \\
BXD16 & 7.517 & 0.017 \\
BXD18 & 9.241 & 0.353 \\
BXD19 & 7.749 & 0.352 \\
BXD20 & 7.674 & 0.241 \\
BXD21 & 8.793 & 0.02 \\
BXD22 & 9.086 & 0.378 \\
BXD23 & 8.852 & 0.558 \\
BXD24 & 9.148 & 0.118 \\
BXD25 & 8.884 & 0.227 \\
BXD27 & 7.531 & 0.13 \\
BXD28 & 8.819 & 0.008 \\
BXD29 & 7.478 & X \\
BXD31 & 7.685 & 0.384 \\
BXD32 & 8.097 & 0.449 \\
BXD33 & 8.472 & 0.743 \\
BXD34 & 9.105 & 0.405 \\
BXD36 & 7.85 & 0.184 \\
BXD38 & 7.408 & 0.275 \\
BXD39 & 9.111 & 0.653 \\
BXD40 & 7.836 & 0.313 \\
BXD42 & 7.668 & 0.259 \\
BXD43 & 7.69 & 0.334 \\
BXD44 & 9.096 & 0.376 \\
BXD45 & 7.705 & 0.102 \\
BXD48 & 7.516 & 0.488 \\
BXD48a (BXD96) & 9.708 & 0.328
\end{tabular}


Table A-2. (continued)

\begin{tabular}{|c|c|c|}
\hline BXD Strain & $\begin{array}{c}\text { Cacna2d1 } \\
\text { Expression }\end{array}$ & SE \\
\hline BXD50 & 9.399 & 0.11 \\
\hline BXD51 & 8.319 & 0.429 \\
\hline BXD55 & 9.159 & 0.439 \\
\hline BXD56 & 9.31 & 0.067 \\
\hline BXD60 & 7.585 & 0.158 \\
\hline BXD61 & 9.035 & 0.14 \\
\hline BXD62 & 7.754 & 0.048 \\
\hline BXD63 & 7.885 & 0.127 \\
\hline BXD64 & 7.812 & 0.026 \\
\hline BXD65 & 9.086 & 0.029 \\
\hline BXD65a (BXD97) & 8.771 & 0.207 \\
\hline BXD65b (BXD92) & 7.708 & 0.129 \\
\hline BXD66 & 8.727 & 0.359 \\
\hline BXD67 & 9.088 & 0.248 \\
\hline BXD68 & 8.456 & 0.634 \\
\hline BXD69 & 7.846 & $\mathrm{x}$ \\
\hline BXD70 & 7.552 & 0.3 \\
\hline BXD71 & 6.91 & 0.239 \\
\hline BXD73 & 9.506 & 0.058 \\
\hline BXD73a (BXD80) & 9.221 & 0.14 \\
\hline BXD75 & 7.627 & 0.312 \\
\hline BXD77 & 8.902 & 0.446 \\
\hline BXD83 & 9.541 & 0.081 \\
\hline BXD84 & 7.828 & 0.031 \\
\hline BXD85 & 7.634 & 0.457 \\
\hline BXD86 & 8.718 & 0.912 \\
\hline BXD87 & 9.207 & 0.078 \\
\hline BXD89 & 7.535 & 0.624 \\
\hline BXD90 & 8.091 & 0.297 \\
\hline BXD98 & 7.854 & 0.59 \\
\hline BXD99 & 8.864 & 0.24 \\
\hline
\end{tabular}




\section{APPENDIX B. SUPPLEMENTAL DATA FOR CHAPTER 3}

Table B-1. Sncg expression in retina of BXD strains

\begin{tabular}{|c|c|c|}
\hline BXD Strain & $\begin{array}{c}\text { Sncg } \\
\text { Expression }\end{array}$ & SE \\
\hline B6D2F1 & 14.175 & 0.106 \\
\hline D2B6F1 & 13.958 & 0.235 \\
\hline C57BL/6J & 13.747 & 0.147 \\
\hline $\mathrm{DBA} / 2 \mathrm{~J}$ & 14.267 & 0.095 \\
\hline BXD1 & 13.772 & 0.071 \\
\hline BXD2 & 13.995 & 0.188 \\
\hline BXD5 & 14.15 & 0.127 \\
\hline BXD6 & 13.513 & 0.359 \\
\hline BXD8 & 13.669 & 0.252 \\
\hline BXD9 & 13.716 & 0.066 \\
\hline BXD11 & 14.413 & 0.085 \\
\hline BXD12 & 13.761 & 0.087 \\
\hline BXD13 & 12.68 & 0.15 \\
\hline BXD14 & 13.71 & 0.135 \\
\hline BXD15 & 12.652 & 0.035 \\
\hline BXD16 & 13.205 & 0.315 \\
\hline BXD18 & 12.84 & 0.124 \\
\hline BXD19 & 13.68 & 0.3 \\
\hline BXD20 & 13.744 & 0.107 \\
\hline BXD21 & 13.488 & 0.211 \\
\hline BXD22 & 14.169 & 0.09 \\
\hline BXD24a & 14.274 & 0.139 \\
\hline BXD27 & 13.907 & 0.117 \\
\hline BXD28 & 13.681 & 0.131 \\
\hline BXD29 & 13.781 & 0.191 \\
\hline BXD31 & 13.065 & 0.147 \\
\hline BXD33 & 13.133 & 0.058 \\
\hline BXD34 & 13.585 & 0.161 \\
\hline BXD36 & 13.753 & 0.126 \\
\hline BXD38 & 13.379 & 0.139 \\
\hline BXD39 & 14.385 & 0.191 \\
\hline BXD40 & 13.284 & 0.159 \\
\hline BXD42 & 13.837 & 0.128 \\
\hline BXD43 & 13.761 & 0.267 \\
\hline BXD44 & 14.406 & 0.17 \\
\hline BXD45 & 13.782 & 0.105 \\
\hline BXD48 & 14.378 & 0.08 \\
\hline BXD48a (BXD96) & 14.363 & 0.08 \\
\hline
\end{tabular}


Table B-1. (continued)

\begin{tabular}{lll}
\hline \multicolumn{1}{c}{ BXD Strain } & $\begin{array}{c}\text { Sncg } \\
\text { Expression }\end{array}$ & SE \\
\hline BXD50 & 14.031 & 0.176 \\
BXD51 & 13.875 & 0.151 \\
BXD53 & 13.847 & 0.089 \\
BXD55 & 13.799 & 0.293 \\
BXD56 & 13.076 & 0.192 \\
BXD60 & 13.698 & 0.208 \\
BXD61 & 14.469 & 0.157 \\
BXD62 & 13.859 & 0.204 \\
BXD63 & 13.717 & 0.173 \\
BXD65 & 13.832 & 0.046 \\
BXD65a (BXD97) & 14.066 & 0.211 \\
BXD65b (BXD92) & 13.901 & X \\
BXD66 & 13.473 & 0.244 \\
BXD67 & 14.07 & 0.227 \\
BXD68 & 13.716 & 0.199 \\
BXD69 & 13.685 & 0.205 \\
BXD71 & 14.327 & 0.472 \\
BXD73 & 13.283 & 0.264 \\
BXD73a (BXD80) & 13.616 & 0.165 \\
BXD73b (BXD103) & 13.552 & 0.14 \\
BXD74 & 13.843 & 0.192 \\
BXD75 & 13.957 & 0.278 \\
BXD77 & 14.361 & 0.147 \\
BXD81 & 14.184 & 0.277 \\
BXD84 & 14.197 & 0.217 \\
BXD85 & 13.768 & 0.187 \\
BXD86 & 13.915 & 0.226 \\
BXD87 & 13.557 & 0.098 \\
BXD90 & 13.706 & 0.127 \\
BXD95 & 13.7 & 0.244 \\
BXD98 & 14.315 & 0.148 \\
BXD99 & 14.356 & 0.111 \\
BXD100 & 13.78 & 0.324 \\
BXD101 & 14.012 & 0.152 \\
BXD102 & 14.074 & 0.227 \\
\hline & &
\end{tabular}


Table B-2. $\quad P f d n 2$ expression in BXD strains retina

\begin{tabular}{lll}
\hline \multicolumn{1}{c}{ BXD Strain } & \multicolumn{1}{c}{ Pfdn2 } & SE \\
& Expression & \\
\hline B6D2F1 & 13.707 & 0.23 \\
D2B6F1 & 13.696 & 0.263 \\
C57BL/6J & 13.628 & 0.215 \\
DBA/2J & 13.843 & 0.235 \\
BXD1 & 13.674 & 0.137 \\
BXD2 & 13.682 & 0.25 \\
BXD5 & 13.265 & 0.309 \\
BXD6 & 13.555 & 0.141 \\
BXD8 & 13.206 & 0.285 \\
BXD9 & 13.405 & 0.081 \\
BXD11 & 13.906 & 0.088 \\
BXD12 & 13.561 & 0.109 \\
BXD13 & 12.492 & 0.104 \\
BXD14 & 13.719 & 0.199 \\
BXD15 & 12.786 & 0.135 \\
BXD16 & 13.714 & 0.408 \\
BXD18 & 12.492 & 0.085 \\
BXD19 & 13.267 & 0.15 \\
BXD20 & 14.136 & 0.108 \\
BXD21 & 13.767 & 0.219 \\
BXD22 & 13.309 & 0.267 \\
BXD24a & 13.952 & 0.277 \\
BXD27 & 14.017 & 0.199 \\
BXD28 & 13.353 & 0.223 \\
BXD29 & 13.626 & 0.135 \\
BXD31 & 13.001 & 0.094 \\
BXD33 & 13.535 & 0.339 \\
BXD34 & 13.238 & 0.298 \\
BXD36 & 13.87 & 0.229 \\
BXD38 & 13.465 & 0.199 \\
BXD39 & 14.415 & 0.196 \\
BXD40 & 13.064 & 0.205 \\
BXD42 & 13.65 & 0.322 \\
BXD43 & 13.57 & 0.497 \\
BXD44 & 14.028 & 0.411 \\
BXD45 & 13.774 & 0.186 \\
BXD48 & 13.53 & 0.108 \\
BXD48a (BXD96) & 13.553 & 0.134 \\
BXD50 & 13.625 & 0.225 \\
BXD51 & 14.319 & 0.193 \\
BXD53 & 13.45 & 0.216 \\
\hline & &
\end{tabular}


Table B-2. (continued)

\begin{tabular}{lcl}
\hline \multicolumn{1}{c}{ BXD Strain } & $\begin{array}{c}\text { Pfdn2 } \\
\text { Expression }\end{array}$ & SE \\
\hline BXD55 & 13.366 & 0.265 \\
BXD56 & 13.302 & 0.194 \\
BXD60 & 13.047 & 0.305 \\
BXD61 & 14.066 & 0.246 \\
BXD62 & 13.371 & 0.314 \\
BXD63 & 13.555 & 0.187 \\
BXD65 & 13.473 & 0.254 \\
BXD65a (BXD97) & 13.891 & 0.177 \\
BXD65b (BXD92) & 13.377 & $\mathrm{x}$ \\
BXD66 & 13.38 & 0.402 \\
BXD67 & 13.701 & 0.208 \\
BXD68 & 13.408 & 0.172 \\
BXD69 & 13.586 & 0.358 \\
BXD71 & 13.792 & 0.477 \\
BXD73 & 13.085 & 0.314 \\
BXD73a (BXD80) & 13.974 & 0.209 \\
BXD73b (BXD103) & 13.268 & 0.1 \\
BXD74 & 13.052 & 0.041 \\
BXD75 & 13.484 & 0.224 \\
BXD77 & 13.966 & 0.165 \\
BXD81 & 13.179 & 0.226 \\
BXD84 & 13.239 & 0.122 \\
BXD85 & 13.546 & 0.245 \\
BXD86 & 13.821 & 0.222 \\
BXD87 & 13.627 & 0.211 \\
BXD90 & 13.458 & 0.353 \\
BXD95 & 13.546 & 0.21 \\
BXD98 & 13.413 & 0.196 \\
BXD99 & 13.897 & 0.293 \\
BXD100 & 13.416 & 0.199 \\
BXD101 & 13.703 & 0.192 \\
BXD102 & 13.382 & 0.238 \\
\hline & &
\end{tabular}


Table B-3. Gene ontology of Cytochrome-c oxidase activity

\begin{tabular}{|c|c|c|c|c|c|}
\hline \multicolumn{6}{|c|}{ Database: molecular function } \\
\hline \multicolumn{6}{|c|}{$\mathrm{C}=16 ; \mathrm{O}=2 ; \mathrm{E}=0.12 ; \mathrm{R}=17.06 ; \operatorname{rawP}=0.0060 ; \operatorname{adj} \mathrm{P}=0.0590$} \\
\hline Index & User ID & $\begin{array}{c}\text { Gene } \\
\text { Symbol }\end{array}$ & Gene Names & $\begin{array}{c}\text { Entrez } \\
\text { Gene }\end{array}$ & Ensembl \\
\hline 1 & ILMN_2657141 & Surfl & Surfeit gene 1 & 20930 & ENSMUSG00000015790 \\
\hline 2 & ILMN_1254971 & Cox6b1 & Cytochrome c oxidase, subunit VIb polypeptide & 110323 & ENSMUSG00000036751 \\
\hline
\end{tabular}

Table B-4. Gene ontology for NADH dehydrogenase activity

\begin{tabular}{|c|c|c|c|c|c|}
\hline \multicolumn{6}{|c|}{ Database: molecular function Name: NADH dehydrogenase activity ID:GO:0003954 } \\
\hline \multicolumn{6}{|c|}{$\mathrm{C}=18 ; \mathrm{O}=2 ; \mathrm{E}=0.13 ; \mathrm{R}=15.17 ; \operatorname{raw} \mathrm{P}=0.0075 ; \operatorname{adj} \mathrm{P}=0.0590$} \\
\hline Index & User ID & $\begin{array}{c}\text { Gene } \\
\text { Symbol }\end{array}$ & Gene Names & $\begin{array}{l}\text { Entrez } \\
\text { Gene }\end{array}$ & Ensembl \\
\hline 1 & ILMN_1220362 & Ndufal 2 & $\begin{array}{l}\text { NADH dehydrogenase (ubiquinone) } 1 \text { alpha } \\
\text { subcomplex, } 12\end{array}$ & 66414 & ENSMUSG00000020022 \\
\hline 2 & ILMN_2985053 & Ndufve 2 & $\begin{array}{l}\text { NADH dehydrogenase (ubiquinone) } \\
\text { flavoprotein } 2\end{array}$ & 72900 & ENSMUSG00000024099 \\
\hline
\end{tabular}


Table B-5. Gene ontology for oxidoreductase activity

\begin{tabular}{|c|c|c|c|c|c|}
\hline \multirow{2}{*}{\multicolumn{6}{|c|}{$\begin{array}{c}\text { Database: molecular function Name: oxidoreductase activity ID:G } \\
\qquad \mathrm{C}=663 ; \mathrm{O}=11 ; \mathrm{E}=4.86 ; \mathrm{R}=2.26 ; \operatorname{raw} \mathrm{P}=0.0095 ; \operatorname{adj} \mathrm{P}=0.0590\end{array}$}} \\
\hline & & & & & \\
\hline Index & User ID & $\begin{array}{c}\text { Gene } \\
\text { Symbol }\end{array}$ & Gene Names & $\begin{array}{c}\text { Entrez } \\
\text { Gene }\end{array}$ & Ensembl \\
\hline 1 & ILMN_2752552 & Sod1 & Superoxide dismutase 1 , soluble & 20655 & ENSMUSG00000022982 \\
\hline 2 & ILMN_2610531 & $S p r$ & Sepiapterin reductase & 20751 & ENSMUSG00000033735 \\
\hline 3 & ILMN_1220362 & Ndufal2 & $\begin{array}{l}\text { NADH dehydrogenase (ubiquinone) } 1 \text { alpha } \\
\text { subcomplex, } 12\end{array}$ & 66414 & ENSMUSG00000020022 \\
\hline 4 & ILMN_2657141 & Surf1 & Surfeit gene 1 & 20930 & ENSMUSG00000015790 \\
\hline 5 & ILMN_1258815 & $J m j d l c$ & Jumonji domain containing $1 \mathrm{C}$ & 108829 & ENSMUSG00000037876 \\
\hline 6 & ILMN_2985053 & $N d u f v 2$ & NADH dehydrogenase (ubiquinone) flavoprotein 2 & 72900 & ENSMUSG00000024099 \\
\hline 7 & ILMN_2512849 & Uqcrh & Ubiquinol-cytochrome c reductase hinge protein & 66576 & ENSMUSG00000063882 \\
\hline 8 & ILMN_1220100 & Ywhae & $\begin{array}{l}\text { Tyrosine } 3 \text {-monooxygenase/ } \\
\text { Tryptophan 5-monooxygenase activation protein, } \\
\text { epsilon polypeptide }\end{array}$ & 22627 & ENSMUSG00000020849 \\
\hline 9 & ILMN_2594149 & Sdhc & $\begin{array}{l}\text { Succinate dehydrogenase complex, subunit } \mathrm{C} \text {, } \\
\text { integral membrane } \\
\text { protein }\end{array}$ & 66052 & ENSMUSG00000058076 \\
\hline 10 & ILMN_1254971 & Cox6b1 & Cytochrome c oxidase, subunit VIb polypeptide 1 & 110323 & ENSMUSG00000036751 \\
\hline
\end{tabular}


Table B-6. Gene ontology for hydrogen ion transmembrane transporter activity

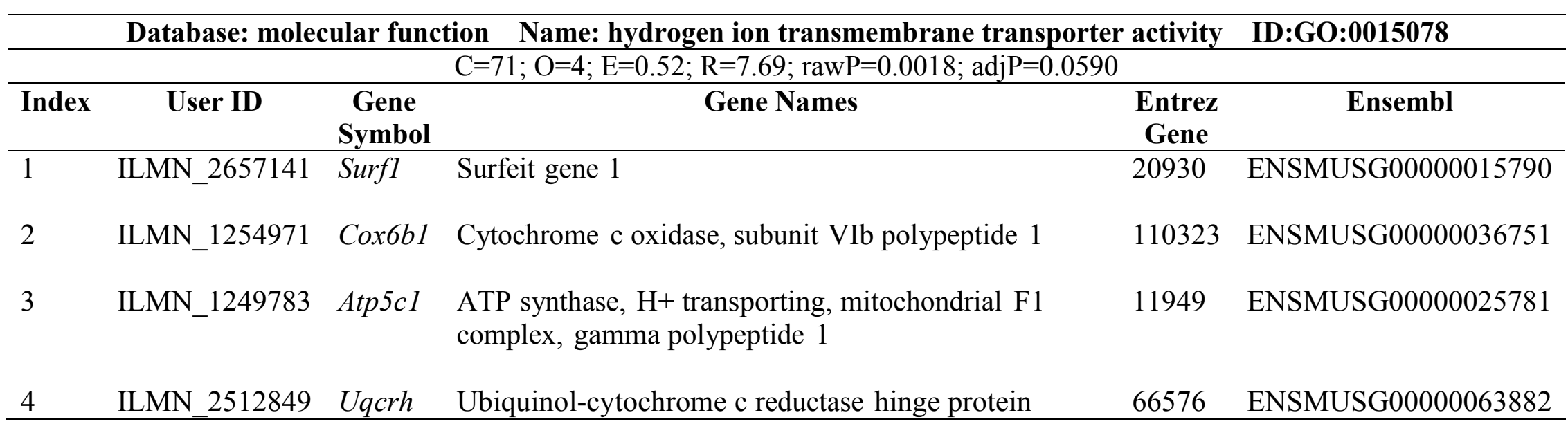


Table B-7. Gene ontology for structural constituent of ribosome

\begin{tabular}{|c|c|c|c|c|c|}
\hline \multicolumn{3}{|c|}{ Database: molecular function } & \multicolumn{3}{|c|}{ Name: structural constituent of ribosome $\quad$ ID:GO:0003735 } \\
\hline \multicolumn{6}{|c|}{$\mathrm{C}=81 ; \mathrm{O}=4 ; \mathrm{E}=0.59 ; \mathrm{R}=6.74 ; \operatorname{rawP}=0.0030 ; \operatorname{adj} \mathrm{P}=0.0590$} \\
\hline Index & User ID & $\begin{array}{c}\text { Gene } \\
\text { Symbol }\end{array}$ & Gene Names & $\begin{array}{c}\text { Entrez } \\
\text { Gene }\end{array}$ & Ensembl \\
\hline 1 & ILMN_1229110 & Mrpl33 & $\begin{array}{l}\text { Mitochondrial ribosomal } \\
\text { protein L33 }\end{array}$ & 66845 & ENSMUSG00000029142 \\
\hline 2 & ILMN_2646163 & Rpl7 & Ribosomal protein L7 & 19989 & ENSMUSG00000043716 \\
\hline 3 & ILMN_2883147 & Mrpl12 & $\begin{array}{l}\text { Mitochondrial ribosomal } \\
\text { protein L12 }\end{array}$ & 56282 & ENSMUSG00000039640 \\
\hline 4 & ILMN_1236904 & Mrpl23 & $\begin{array}{l}\text { Mitochondrial ribosomal } \\
\text { protein L23 }\end{array}$ & 19935 & ENSMUSG00000037772 \\
\hline
\end{tabular}




\section{APPENDIX C. SUPPLEMENTAL DATA FOR CHAPTER 4}

Table C-1. List of primers used for gene expression analysis as a component of the validation of RGC enrichment protocol

\section{Gene symbol Taqman $^{\circledR}$ Gene Expression assays (Primers)}

\begin{tabular}{ll}
\hline Abca8a & Mm00462440_m1 \\
Aldh1al & Mm00657317_m1 \\
Aqp4 & Mm00802131_m1 \\
Calb2 & Mm00801461_m1 \\
Cd68 & Mm03047340_m1 \\
Gad2 & Mm00484623_m1 \\
Hprt & Mm01545399_m1 \\
Lhx1 & Mm01297482_m1 \\
Lim2 & Mm00624623_m1 \\
Nrl & Mm00476550_m1 \\
Ntrk1 & Mm01219406_m1 \\
Pcp4 & Mm00500973_m1 \\
Pov4f1 & Mm02343791_m1 \\
Prdx6 & Mm00725435_s1 \\
Prkca & Mm00440858_m1 \\
Prox1 & Mm00435969_m1 \\
Pvalb & Mm00443100_m1 \\
Rbpms & Mm02343791_m1 \\
Rom1 & Mm00436364_g1 \\
Rpe65 & Mm00504133_m1 \\
Slcla3 & Mm00600697_m1 \\
Slc6a9 & Mm00433662_m1 \\
Sncg & Mm00488345_m1 \\
Tubb3 & Mm00727586_s1 \\
Vim & Mm01333430_m1 \\
\hline
\end{tabular}




\section{VITA}

Sumana Rameshbabu Chintalapudi was born in Ahmedabad, Gujarat, India in 1987, the daughter of Ramesh Babu Chintalapudi and Usha Rani Chithirala. After completing her degree at Kendriya Vidhalaya, Ahmedabad, India in 2005, she entered the St. Xavier's College, Gujarat, India and received a dual degree of Bachelor of Science and Diploma in Bioinformatics in May, 2008. In 2008, Sumana entered the Biomedical Genetics Program at Vellore Institute of Technology, Tamil Nadu, India to pursue her Master's degree. She performed her Master's Thesis at Department of Physiology, University of Tennessee Health Science Center (UTHSC). She graduated with a Master's of Science degree (Thesis) in May 2010. In 2010 she joined UTHSC as a doctoral student in Integrated Program in Biomedical Sciences, Neuroscience Track.

During her time at UTHSC, Sumana has made several presentations, including poster and podium, published papers in peer reviewed journals. She has been awarded Best Poster Awards several times at UTHSC. She has presented posters at The Association for Research in Vision and Ophthalmology (ARVO) several times during her doctoral studies. She has been awarded John Austian Student Enrichment Fund and Neuroscience Travel Awards for presenting her research at annual ARVO conferences. In 2013, Sumana was selected as Commercialization Analyst Intern at University of Tennessee Research Foundation (UTRF). She successfully handled several responsibilities, including but not limited to, prior art research, screening and evaluation of technologies, market and industry analyses to investigate novelty of the technologies for licensing. She assisted in $\mathrm{Ad} \mathrm{Hoc}$ projects in marketing and licensing of cutting-edge technology developed at UTHSC. In February 2015, Sumana was invited for podium presentation in Young Investigator Platform Session at Association for Ocular Pharmacology and Therapeutics. In May 2016 Sumana was awarded Joseph M. and Eula C. Lawrence Travel Grant by Retina research foundation to present a poster at ARVO at Seattle, WA. Her poster was ranked Top 5\% and she was also selected as 1 of 79 trainees for Members in Training (MIT) Poster Competition. After having successfully defended her dissertation in April 2016, Sumana will be awarded her doctoral degree in August 2016. 